\begin{abstract}
Title of Document:

MODELING AND SIMULATION OF SHOCK

AND DROP LOADING FOR COMPLEX

PORTABLE ELECTRONIC SYSTEMS.

Alex F. Askari Farahani, M. S. Engineering, 2009

Directed By: $\quad$ Professor Abhijit Dasgupta, Department of

Mechanical Engineering
\end{abstract}

In this study, the transient response of electronic assemblies to mechanical loading encountered in drop and shock conditions are investigated. Many manufactures face design challenges when evolving new designs for high strain-rate life-cycle loading. Examples of high strain-rate loading include drop events, blast events, vibration, ultrasonic process steps, etc. New design iterations invariably bring new unexpected failure modes under such loading and costly trial-and-error design fixes are often necessary after the product is built. Electronics designers have long sought to address these effects during the design phase, with the aid of computational models. However, such efforts have been difficult because of the nonlinearities inherent in complex assemblies and complex dynamic material properties. Our goal in this study is to investigate the ability of finite element models to accurately capture the transient response of a complex portable electronic product under shock and drop loading. The portable electronic assembly in this study consists of a circuit card assembly in a plastic housing. Dynamic loading, consisting of broad-band vibration 
tests and shock tests on an electrodynamic shaker, and drop tests on a commercial drop-tower are applied to the test system as well as to its constituent sub-assemblies. The tests at the sub-assembly level are used to calibrate the dynamic response of the individual constituents. The nonlinear interactions due to dynamic contact between these sub-assemblies is then investigated through shock and drop testing at the system level. Finite element models of the system are generated and calibrated at the subsystem level with results of random vibration and shock tests. The contact mechanics are then parametrically investigated with the finite element model by comparing with the drop response of the full product. The parametric study consists of sensitivity studies for different ways to model soft, non-conservative contact, as well as structural damping of the sub-assembly under assembly boundary conditions. The long-term goal of this study is to demonstrate a systematic modeling methodology to predict the drop response of future portable electronic products, so that relevant failure modes can be eliminated by design iterations early in the design cycle. 


\title{
MODELING AND SIMULATION OF SHOCK AND DROP LOADING FOR COMPLEX PORTABLE ELECTRONIC SYSTEMS
}

\author{
By \\ Alex Farbod Askari Farahani. \\ Thesis submitted to the Faculty of the Graduate School of the \\ University of Maryland, College Park, in partial fulfillment \\ of the requirements for the degree of \\ Master of Science in \\ Mechanical Engineering \\ 2009
}

Advisory Committee:

Professor Abhijit Dasgupta, Chair

Professor Donald Barker

Professor Bongtae Han 
(C) Copyright by

Alex Farbod Askari Farahani

2009 


\section{Dedication}

To my family and friends. 


\section{Acknowledgements}

Ever since I was a little kid I always wanted to know what it would be like to graduate with an M.S. degree. I cannot believe that my boyhood dream has come true. I would like to thank my advisor, Professor Abhijit Dasgupta. I would have not been at this point in my life, if it had not been for all his support and guidance.

I would also like to send my special thanks to Dr. Moustafa Al-Bassyiouni for all his helpful suggestions and insight both in my experimental and computational work. He stayed late at work in many different occasions so I could get my experiments to work, and for that I am very thankful.

In addition, special thanks to Cholmin Choi, Yasir Majeed, Koustav sinha, Danial Farley, Gayatri, Lynn Munday, Shaughn London, Arnaud Delye, Stuart Douglas, Joe Varghese, and all other CALCE former and current employees not only for their assistance but also for making my graduate school experience a memorable one. 


\section{Table of Contents}

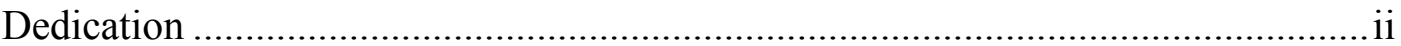

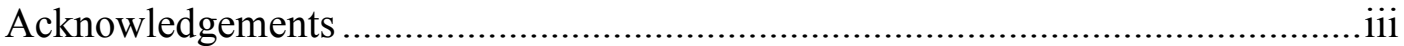

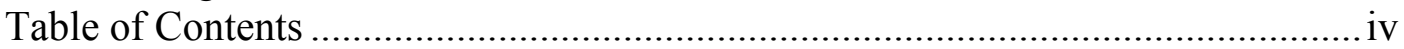

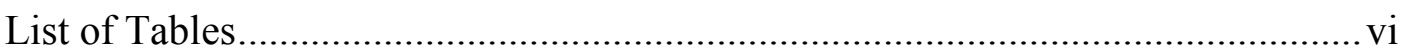

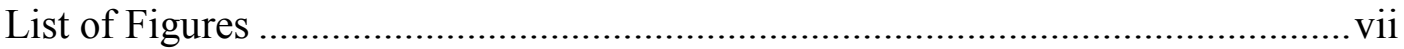

Chapter 1 Introduction ................................................................................ 1

$1.1 \quad$ Problem Statement and Objectives: ........................................................ 2

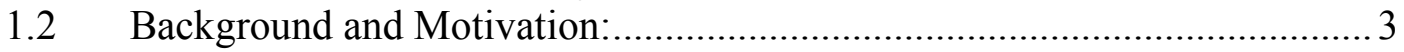

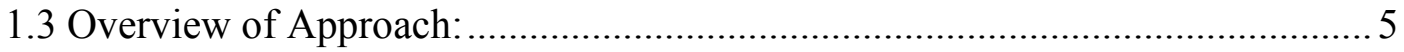

Chapter 2 Shock and Dynamic Loading in Portable Electronics................................ 8

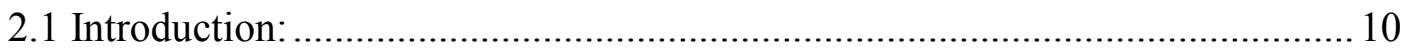

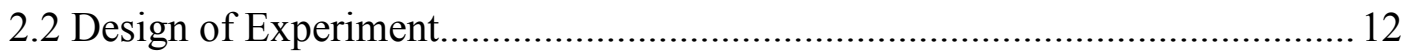

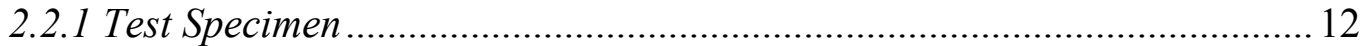

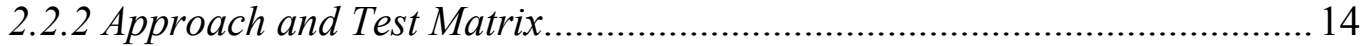

2.3 Shock Test Set Up .................................................................................... 16

2.3.1 Test Set Up for Broad Band and Shock Loading of Clamped PWB ........... 16

2.3.2 Test Set Up for Broad Band Excitation of Spring-mounted PWB .............. 20

2.3.3 Test Set Up for Broad Band Excitation of Plastic Housing ........................2 21

2.3.4 Test Set Up for Broad Band Excitation of Complete Product Assembly ...24

2.3.5 Shock Test Up of Complete Product Assembly ......................................... 28

2.4 Drop Test Set Up ........................................................................................ 29

2.4.1 Drop Test Set Up for Clamped PWB and Full Product Assembly ..............30

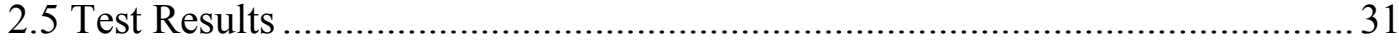

2.5.1 Broad Band and Shock Test Results........................................................ 31

2.5.1.1 Broad Band Response of Clamped and Spring-mounted PWBs ..........31

2.5.1.2 Shock Response of Clamped PWB .................................................... 32

2.5.1.3 Empty Case Broad Band Test Results ................................................. 38

2.5.1.4 Broad Band Test Results for Full Product Assembly .......................... 42

2.5.1.5 Results of Shock Test on Full Product Assembly ................................ 44

2.5.2 Drop Test Results ................................................................................ 47

2.5.2.1 Drop Test Results for Clamped PWB ............................................. 47

2.5.2.2Full Product Drop Test Results for Full Product Assembly ................. 52

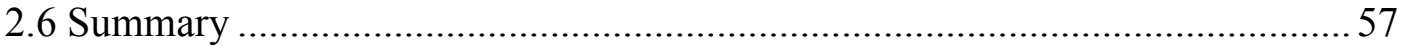

Chapter 3 Modeling For Shock and Dynamic Loading in Portable Electronic

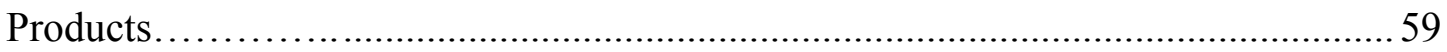

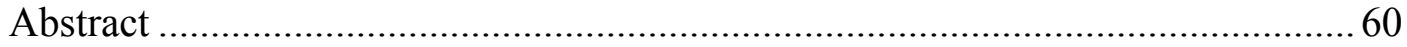

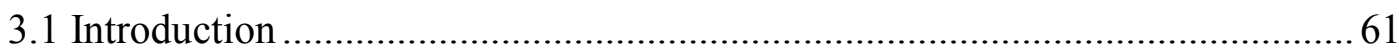

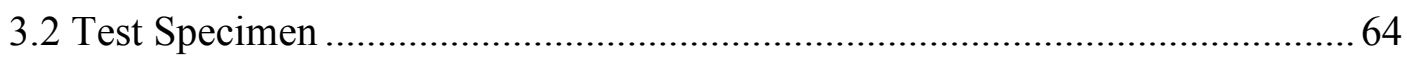

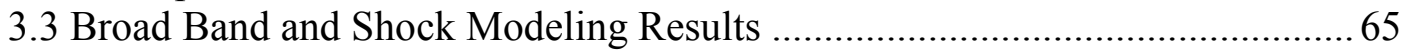


3.3.1 Finite Element Modeling of Clamped PWB .............................................6 65

3.3.2 Finite Element Model of Spring-Mounted PWB ..................................... 73

3.3.4 Modal Analysis of Empty Housing ............................................................ 75

3.4.5 Broad Band Simulation of Full Product Assembly .................................. 84

3.4.6 Shock Simulation of Full Product Assembly ........................................... 87

3.5 Drop Test Modeling Results ........................................................................... 90

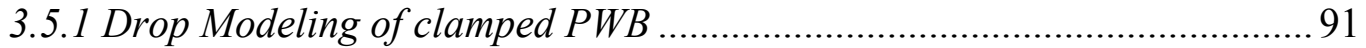

3.5.2 Drop Simulation of Full Product Assembly .............................................. 95

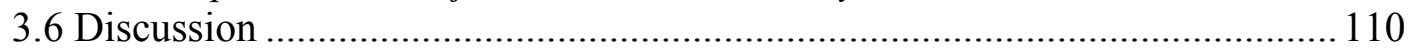

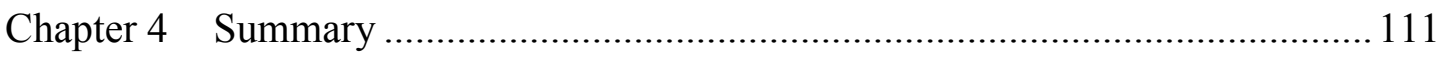

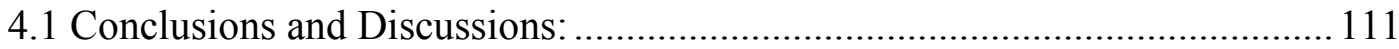

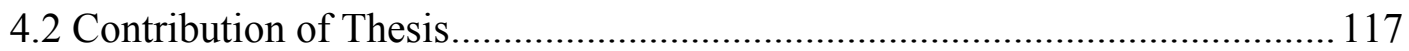

4.3 Future Work ........................................................................................ 118

Appendix A Dimensions of the Product ...................................................... 120

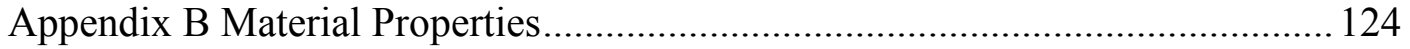

Appendix C Shock Response of Spring-mounted PWB ................................... 125

Appendix D Finite Element Model of Spring-Mounted PWB........................ 128

Appendix E Test Set Up for Mechanical Property of Housing Material .............. 130

Appendix F Test Set Up for Mechanical Property of Housing Material .............. 132

Appendix G Finite Element Modal Analysis of Cantilever Beam for Housing

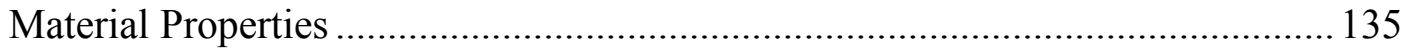

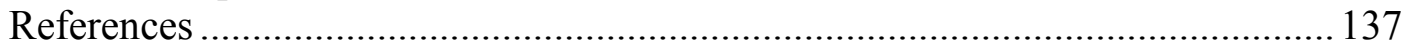




\section{List of Tables}

Table 3-1- Illustrates the effect of contact stiffness on the range of the first cycle of the strain history. Strain is measured at point in the 1 direction as shown in Figure $2-47$. 100

Table 3-2 Illustrates how strain range of the first cycle of the strain history varies as a function of critical damping fraction. Strain is measured at point in the 1 direction as shown in Figure 2-47.

Table B-1 FR4 Material properties ........................................... 124 Table B-2 Modified FR4 material properties used in the simulations of Part II of the

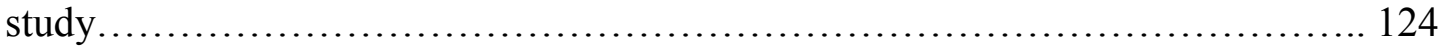




\section{List of Figures}

Figure 1-1 One of the earlier versions of the product under study ............................... 4

Figure 1-2 Au-plated leaf spring .....................................................................

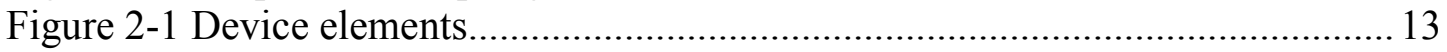

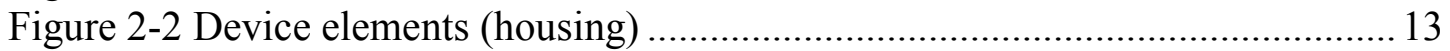

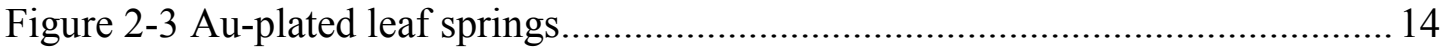

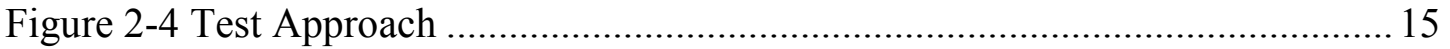

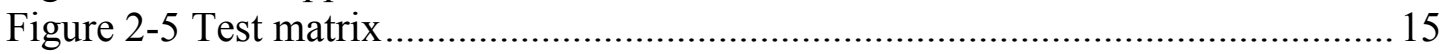

Figure 2-6 Electro dynamic shaker with test specimen............................................ 17

Figure 2-7 Bare board shock test set up. The PWB is fixed at points 1 and 2.

Accelerometers A and B measure the fixture and PWB response. Accelerometer C controls the shaker excitation. Strain gages are labeled with red dotted circles......... 18

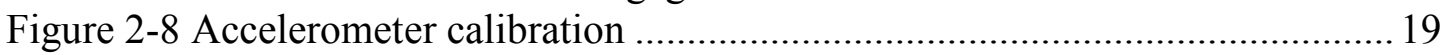

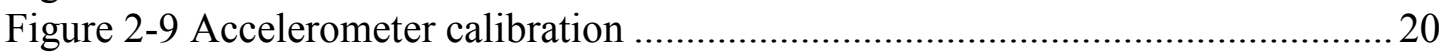

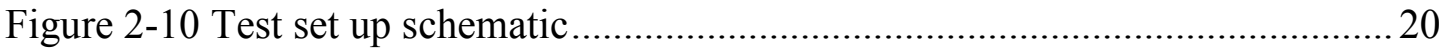

Figure 2-11 Spring-mounted board test set up- The PWB is fixed at points 1 and 2. Accelerometers A and B measure the fixture and PWB response. Accelerometer $\mathrm{C}$ controls the shaker excitation. Strain gages are labeled with red dotted circles.........21

Figure 2-12 Test set up for broad band test on housing. Accelerometers 1 and 2 monitor the response of empty plastic housing. Accelerometer 3 controls the shaker

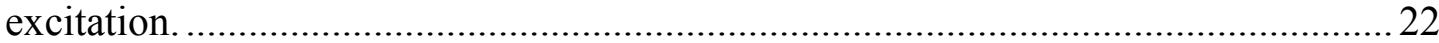

Figure 2-13 First two natural modes of the empty housing when the bottom of the

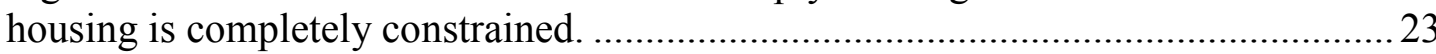

Figure 2-14 First two natural modes of the empty housing when the bottom of the

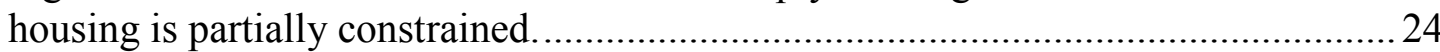

Figure 2-15 Test set up for broad band excitation of the full product and plastic empty housing. The figure illustrates the clamping mechanism used to fix the product to the fixture. The Accelerometer $r$ measures the response of the clamping finger to ensure its natural frequencies do not coincide with those of the full product. ........................26 Figure 2-16 Test set up for the broad band test on empty plastic housing. Accelerometer $\mathrm{H}$ is placed on the same location as the accelerometer II shown in Figure 2-17 and measures the response of the plastic housing. Accelerometer F measures the response of the fixture. ........................................................................ 27 Figure 2-17 Test set up for broad band test on full product assembly. Accelerometer I monitors the fixture response. Accelerometer II and III measure the plastic housing and PWB response.

Figure 2-18 Full product shock test set up- Two strain gages are mounted on the board at Points 1 and 2 to monitor the board response. Strain is measured along the $\mathrm{x}$ direction. Accelerometer A and B monitor the response of the fixture and PWB

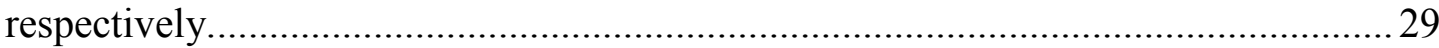

Figure 2-19-Lansmont Drop Tester......................................................................... 30

Figure 2-20 Frequency response function of clamped PWB ...................................... 32

Figure 2-21 Frequency response function of spring-mounted PWB........................... 32

Figure 2-22 Shock response of clamped PWB...………………………………......... 33

Figure 2-23- Acceleration magnification factor for clamped PWB ............................. 34 
Figure 2-24 Flexural strain response $\varepsilon_{\mathrm{xx}}$ on clamped PWB at the location shown in the

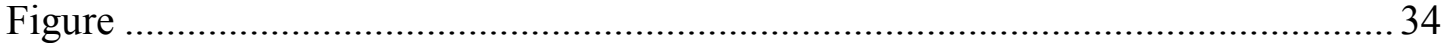
Figure 2-25 FFT response of the strain Gage, which is marked with a red Circle in Figure 2-24, to the half sine shock loading on clamped PWB. Frequency response function of the accelerometer on the free end of the PWB as shown in Figure 2-7. .. 36 Figure 2-26- Amplitude Density Spectrum of Half-Sine Wave Pulse of Duration $\pi / w_{0}$

Figure 2-27 FFT of the fixture motion in response to the half sine shock loading ..... 38 Figure 2-28 Frequency response function of the fixture used for broad-band test of the empty housing. The accelerometer is placed on the base of the fixture as shown in Figure 2-12. Due to the fixture excitation peaks that occur after $2000 \mathrm{~Hz}$, all test data will be ignored in the region marked with a red dotted rectangle.

Figure 2-29 Frequency response function of the empty case at the two accelerometer

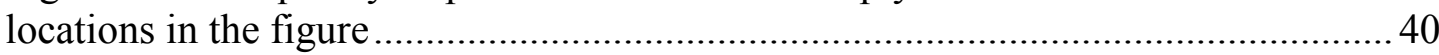
Figure 2-30 Phase Plot between Top and Bottom Accelerometers............................ 41 Figure 2-31 Frequency response function of the empty housing with clamping fingers versus the empty housing without the fingers. The accelerometer location for the case with the clamping fingers is shown in Figure 2-16. The accelerometer location for the case without the clamping fingers is at point $\mathrm{T}$ as shown in Figure 2-29. 42 Figure 2-32 Frequency response function of the clamping fingers at the accelerometer location shown in Figure 2-15.

Figure 2-33 Frequency response function of the full product at the following accelerometer locations: the blue curve represents the accelerometer on the plastic housing. The red curve represents the accelerometer on the PWB. For exact accelerometers' locations on the PWB and plastic housing refer to Figure 2-17 ...... 44 Figure 2-34 Fixture acceleration VS PWB acceleration response measured at location

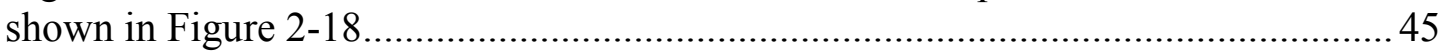

Figure 2-35 Product shock test repeatability ..................................................... 45 Figure 2-36 FFT of the fixture motion in response to the half sine shock loading. The notches of the frequency response function occur at 250, 410580,740 , and $910 \mathrm{~Hz}$

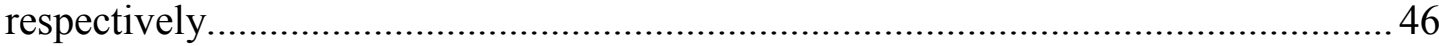
Figure 2-37 Fixture acceleration and PWB acceleration for drop test on clamped PWB

Figure 2-38 Acceleration magnification factor for drop test on clamped PWB ......... 48

Figure 2-39- Bare Board acceleration response can be described with a normal distribution with mean and standard deviation values of $2252 \mathrm{G}$ and $217 \mathrm{G}$, respectively.

Figure 2-40- Strain response at point A measured in the $\mathrm{x}$-direction, during drop test

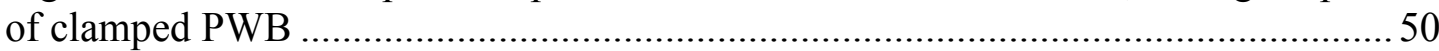

Figure 2-41 FFT response of the drop table fixture to the half sine drop loading ......51 Figure 2-42- FFT response of strain gage in Figure 2-40 to half sine drop loading of

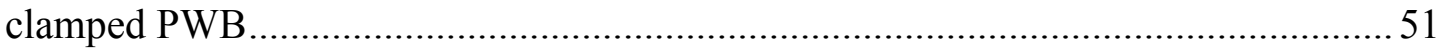
Figure 2-43 Frequency response function of the accelerometer on the free end of the

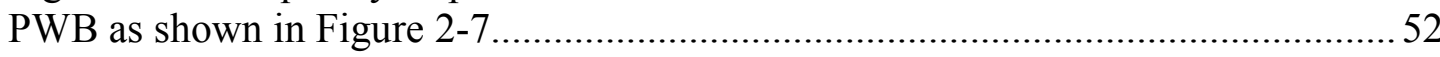
Figure 2-44 Transfer function between peak values of PWB acceleration and fixture acceleration in drop test of full product assembly. 
Figure 2-45 Transfer Function between Peak Values of PWB Strain and PWB Acceleration

Figure 2-46- PWB Acceleration Vs Fixture Acceleration in Product Drop Test. The exact locations of the accelerometer are shown in Figure 2-18 ...............................5 54

Figure 2-47 PWB strain Response at point 2 in product drop test ............................. 54

Figure 2-48 Illustrates the repeatability of the PWB strain response measured at point 2 as shown in Figure 2-47 for 5 different trials. Strain is measured in the xx direction.

Figure 2-49 Illustrates the repeatability of the PWB acceleration response measured at point 2 as shown in Figure 2-47 for 5 different trails. ............................................ 56 Figure 2-50 FFT response of the strain gage on the PWB, shown in Figure 2-47, to drop loading. Acceleration frequency response of the PWB measured at the location

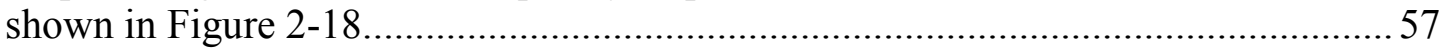
Figure 3-1- Clamped PWB meshed geometry. The PWB is fixed at points 1 and 2. Accelerometer and LEDs masses are added at their designated locations..................66 Figure 3-2- The first two mode shapes of the clamped PWB with $20 \%$ increase in

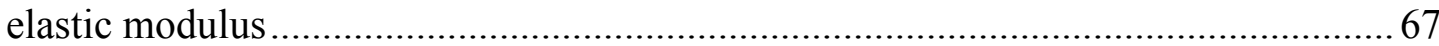

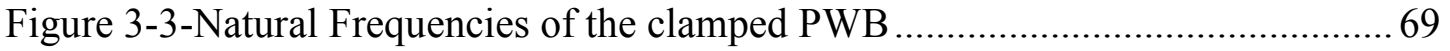

Figure 3-4- FEA input pulse applied at points 1 and 2 .......................................... 70

Figure 3-5 Acceleration at the free end of the clamped PWB. Acceleration is measured in the out-of-plane direction- FEA VS. Experiment ............................... 71 Figure 3-6 Strain at the post of the clamped PWB. Strain is measured in the $x x$ direction as shown in Figure 2-24- FEA VS. Experiment-.................................... 71 Figure 3-7- FFT response of strain gage mounted at the post of the clamped PWB shown in Figure 2-24- FEA VS Experiment ...................................................... 72 Figure 3-8 Frequency response function of the PWB measured at the accelerometer

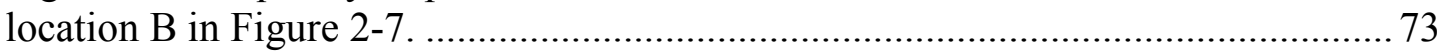

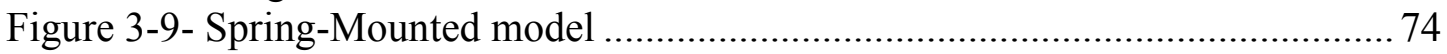
Figure 3-10- Natural Frequencies of the Spring Loaded Board ............................... 75 Figure 3-11- Actual device design versus simplified FEA model. (I) Bottom housing of actual device (II) Bottom housing of FEA model.............................................. 76 Figure 3-12- Actual device design versus simplified FEA model- Top Housing. (I) and (III) Top housing of actual device (II) and (IV) Top housing of FEA Model...... 77 Figure 3-13-Stiffened areas to represent the stiffener ribs of the belt clip on the top

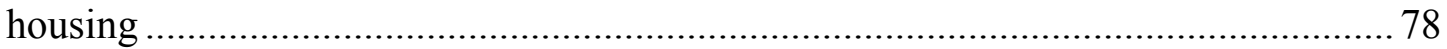

Figure 3-14- Cross sectional area stiffener ribs of the belt clip ............................. 79 Figure 3-15- Cross sectional area stiffener ribs of the belt clip ............................... 79 Figure 3-16- Cross sectional area stiffener ribs of the belt clip ............................... 80

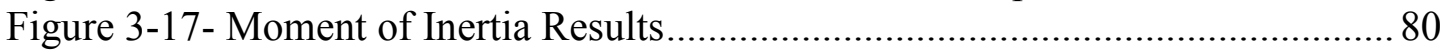
Figure 3-18 Battery Case Geometry vs. Model....................................................... 81 Figure 3-19- FEA model of empty case assembly- Accelerometer masses are added to the model as distributed masses as shown in the figure. Acceleration is measured in

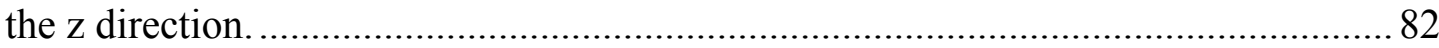
Figure 3-20 Dynamic mode shapes for the empty housing................................... 83 Figure 3-21- Frequency response function of the empty housing measured the accelerometer $\mathrm{T}$ shown in Figure 2-12. 
Figure 3-22 The red crosses indicate the areas at the which the PWB is fixed to roughly simulate the boundary conditions of the PWB inside the full product........ 84 Figure 3-23- Mode shapes and natural frequencies of the PWB when subjected to the constraints similar to that of experienced in the actual device.................................. 86

Figure 3-24- Full Product Assembly ................................................................... 87

Figure 3-25- Full product FEA results when the board is not fixed to the top housing caps. (I) illustrates the contact stresses around the bottom housing post caused by impact between the guide post on the housing and the PWB guide hole. (II) Demonstrates the unrealistic accelerations predicted by the FEA model due to the

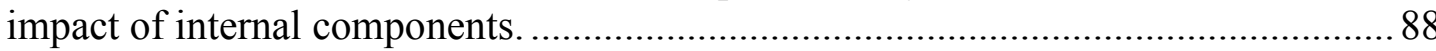
Figure 3-26- Shock test FEA results VS. Experiment results. The acceleration is measured on the PWB in the $\mathrm{z}$ direction as shown in Figure $3-24$.......................... 90 Figure 3-27-Acceleration at the free end of the clamped board as shown in Figure 2-39- FEA VS. Experiment.

Figure 3-28 Strain at the post of the clamped PWB as shown in Figure 2-40-FEA vs.

Experiment

Figure 3-29- Acceleration at the Free End of the Bare Board - FEA VS. Experiment. $\alpha=175 \mathrm{Rad} / \mathrm{s}$ for the case shown..

Figure 3-30- Strain at the post of the clamped PWB- FEA VS. Experiment. $\alpha=175$

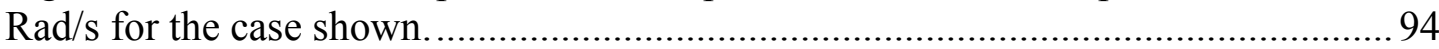

Figure 3-31- Strain FFT Response- FEA VS Experiment ........................................ 94

Figure 3-32- Frequency response function of the PWB measured at the accelerometer location shown in Figure 2-7.

Figure 3-33 (I) Contact stresses at a node next to the tapered post when hard contact is defined. (II) Contact stresses at a node next to the tapered post when soft contact is defined.

Figure 3-34- Comparison of the experimental acceleration histogram with those of the FEA models with soft and hard contact properties. 98 Figure 3-35-Comparison of the experimental acceleration response with those of the FEA models with soft and hard contact properties. 98

Figure 3-36 Comparison of the experimental strain histogram with those of the FEA models with soft and hard contact properties.

Figure 3-37- Comparison of the experimental strain response of the PWB with those of the FEA models with soft and hard contact properties. 100

Figure 3-38 Illustrates the effect of contact stiffness on strain range variation, calculated from the first peak and valley of strain profiles. Strain is measured at point in the 1 direction as shown in Figure 2-47.

Figure 3-39 Illustrates the normalized RMS strain and the strain range values based on various contact stiffness. Strain is measured at point 2 in the $\mathrm{x}$ direction as shown in Figure 2-47. RMS and Strain range values are normalized with respect to hard contact.

Figure 3-40 Illustrates the normalized RMS acceleration and the acceleration range values based on various contact stiffness. RMS and acceleration range values are normalized with respect to hard contact. Acceleration is measured on the PWB according to Figure 2-18. 
Figure 3-41 Illustrates the effect of fraction of critical damping on strain range of the first cycle. Strain is measured at point 2 in the $\mathrm{x}$ direction as shown in Figure 2-47.

Figure 3-42- Illustrates the RMS of the strain and acceleration profiles, as well as the acceleration range of the first cycle based on various fraction of critical damping values. All the values are normalized with respect to hard contact. ........................ 104 Figure 3-43 Illustrates the effect of Raleigh damping on strain range of the first cycle. Strain is measured at point 2 in the $\mathrm{x}$ direction as shown in Figure 2-47. The Raleigh damping factors $\alpha_{1}, \alpha_{2}$ belong to the PWB and the plastic housing respectively...... 106 Figure 3-44 Illustrates the acceleration and strain RMS based on various Raleigh damping values. The Raleigh damping factors $\alpha_{1}, \alpha_{2}$ belong to the PWB and the plastic housing respectively.

Figure 3-45 Drop test FEA results VS. Experiment results- Strain is measured at point 2 in the " 1 " direction as shown in Figure 2-47.

Figure 3-46 Drop test FEA results VS. Experiment results. Acceleration is measured on the PWB in the out-of-plane direction. Refer to Figure 2-18 for exact location of the Accelerometer.

Figure 3-47- FTT response of the accelerometer on the PWB shown in Figure 2-18

vs. FEA results.

Figure 3-48 FTT response of the strain gage at point 2 as shown in in Figure 2-47 vs.

Experiment results.

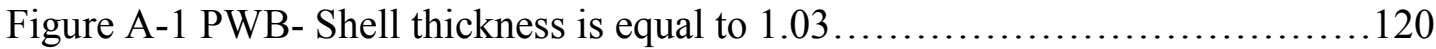

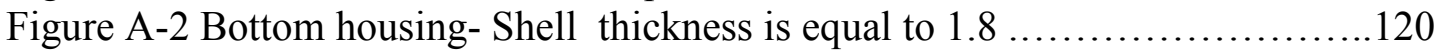

Figure A-3 Bottom housing top view..................................... 121

Figure A-4 Bottom housing A) Rib B) Tapered post........................... 121

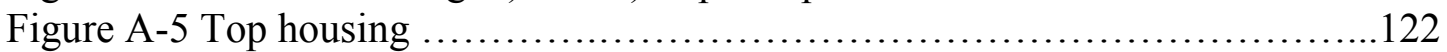

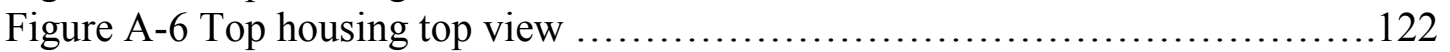

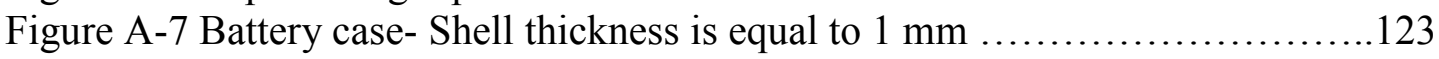

Figure A-8 Battery case top view ....................................... 123

Figure C-1 Acceleration magnification factor for spring-mounted PWB ................ 125

Figure C-2 Fixture acceleration VS spring-mounted board acceleration response

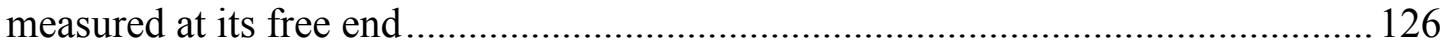

Figure C-3 Strain history $\varepsilon_{\mathrm{yy}}$ at the post of the spring-mounted board .................... 126

Figure C-4 Frequency response function of accelerometer B in Figure 2-11 in response to the half sine shock loading. FFT response of the strain gage, shown in Figure C-3. 


\section{Chapter 1 Introduction}

In the past two decades the electronics industry has undergone tremendous changes. An enormous amount of effort has gone into developing light weight and relatively small personal electronic devices to meet an ever-increasing market demand. Electronic products, during the life cycle from manufacturing plants to endusers, are subjected to various types of dynamic loading mainly due to transportation and handling. In addition, it is common for portable electronic devices to be dropped accidently during usage. As a result, engineers have always faced an immense challenge to design robust and reliable products that are sufficiently rugged and shock resistant. One of the main challenges that manufacturers encounter when designing new products is that the dynamic response of the product is highly sensitive to the slightest change in the design of the system, resulting in unexpected failure modes. One of the most common ways to overcome this problem is by implementing a costly and time-consuming trial and error process. In this process, a product is tested on the field to detect potential failure modes. Once a failure is observed, manufacturers have to go back and redesign parts or the entire system to prevent the failure from reoccurring. This lengthy process could take several iterations and significantly lengthen the product development cycle.

With the invention of super-fast computers, researchers, however, have shifted their attentions towards computational models such as finite element models, to detect failure modes early on in the design phase before the product is built. However, such efforts have been difficult because of the nonlinearities inherent in complex assemblies and because of the uncertainties introduced by complex dynamic material 
properties. This study tries to utilize a systematic building-block approach to overcome some of the difficulties encountered in modeling of portable electronic devices. In chapter 2 , the portable electronic device under study, including its main constituent components, is subjected to various shock and drop loading profiles to acquire knowledge of how the sub-assemblies in these electronic assemblies respond to various operational and environmental dynamic loads, both independently and as a whole in the product. In Chapter 3, ABAQUS ${ }^{\mathrm{TM}}$ [16] Explicit and standard are used to create finite element models for the product under the study, as well as for its subassemblies. The input shock and vibration profiles used during the tests are employed as boundary conditions for the finite element models. Additionally, the modeling results are calibrated with respect to experimental measurements. The goal is to assess the ability to accurately model the dynamic behavior of a complex system if the properties of the sub-assemblies are known.

\subsection{Problem Statement and Objectives:}

The objective of this study is to investigate the ability of finite element models to accurately predict the transient response of complex assemblies used in portable electronic products, when subjected to shock and drop loading conditions. This is accomplished by performing a combination of simple shock and drop tests on the full

assembly, as well as on its main components, such as printed wiring board, spring contacts, and plastic housing. Finite element modeling is carried out using a progressive hierarchical multi-step technique in which material properties and 
boundary conditions of individual subassemblies are calibrated from simple shock and vibration tests prior to proceeding to drop simulation of the full product.

\subsection{Background and Motivation:}

As shown in Figure 2-4 and Figure 2-5, the portable product investigated in this study is divided into the following parts and sub-assemblies for the purposes of this study:

- Main PWB
a. Three LEDs
b. A microphone and its housing
c. Two Au-plated leaf springs

- Metal charge contact pins (2 pins, one on each side)

- Top and bottom plastic housing

- Battery and plastic battery case inside the housing

A detailed description of the product is provided in Section 2.2. 


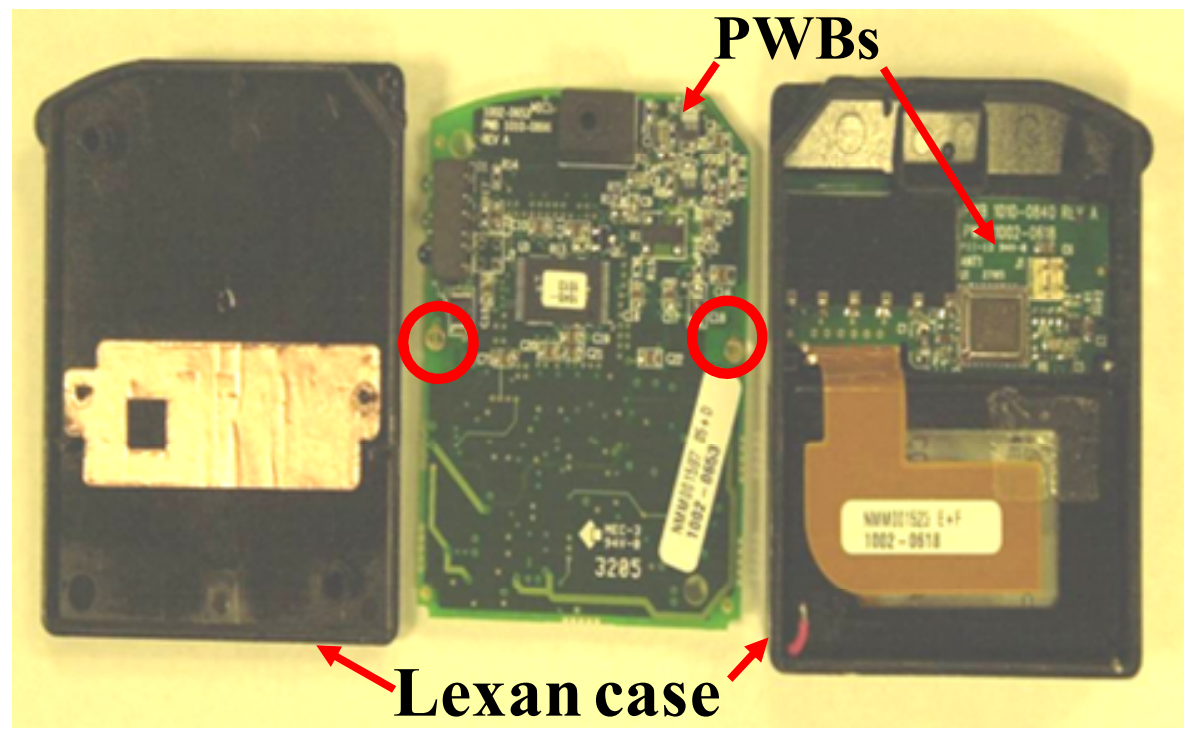

Figure 1-1 One of the earlier versions of the product under study

Figure 1-1 illustrates one of the earlier versions of the product. The design of this product has undergone several revisions since it was first introduced to the market. For instance, the number of the PWBs was reduced from two to one. Another major change in the design of the product was replacing the solder joints between the two charge pins and the PWB (marked with red circles in Figure 1-1), with two Auplated leaf spring contacts, shown in Figure 1-2. The purpose of the springs was to prevent failures of fragile components on the PWB by mechanically isolating it from energy transmitted by the solder joint from the charge pins during ultrasonic welding of the plastic housing as well as during accidental drops. The spring constants are obtained from an extensive study reported elsewhere in the literature. 


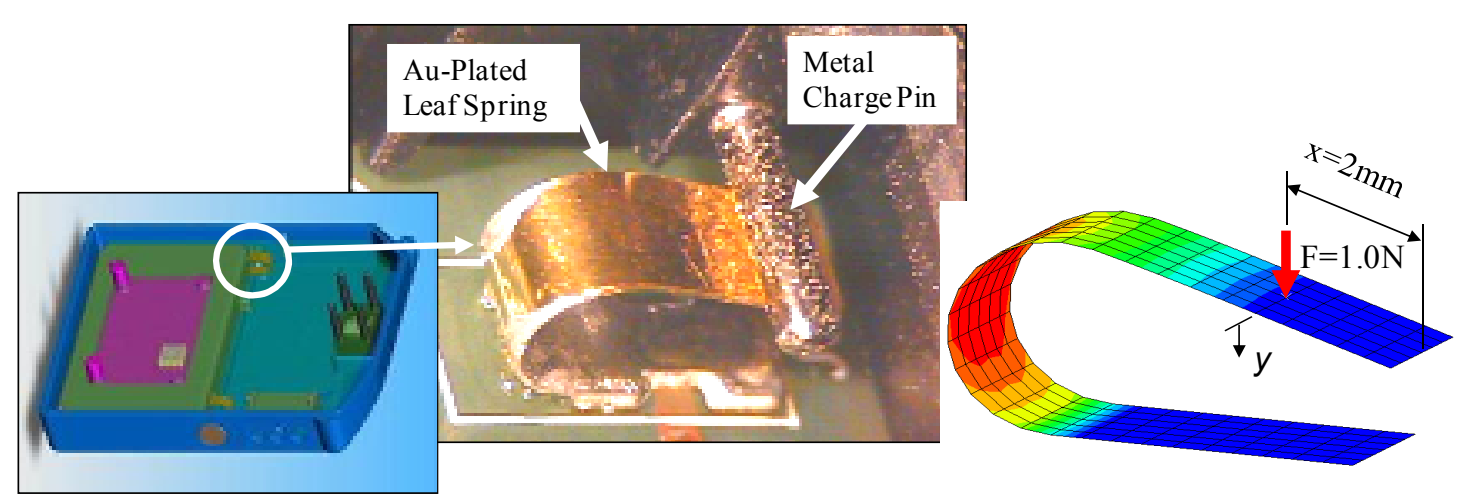

Figure 1-2 Au-plated leaf spring

As discussed earlier, finite element models provide a good alternative to expensive drop testing of portable electronic products to study their dynamic behavior. Therefore, the goal is to develop a finite element model that has sufficient fidelity to accurately predict the transient response of the product when subjected to dynamic loading. This finite element model could be very advantageous in the development of robust products in the future. Furthermore, the same modeling techniques can be extended to other portable electronic devices such as cell phones, PDAs and so forth. Finite element models, however, have posed their own challenges for scientists due to the uncertainties introduced by nonlinearities inherent in complex assemblies and by complex dynamic material properties. Due to the complex nature of product-level drop simulation, assembly-level qualification is currently done mostly with PWB-level testing and simulations, as in JEDEC JESD22-B111 [21]. While many researchers have reported a qualitative ability for such modeling, further work is still needed to demonstrate good quantitative agreement.

\subsection{Overview of Approach:}

The challenge in system-level modeling of drop loading is that discrepancies between simulation and experimental results are difficult to trace back to the source 
of the problem. Therefore, troubleshooting is very difficult. As a result, in this study the full system is divided into its constituent subsystems as shown in Figure 2-4 and Figure 2-5. To eliminate some of the nonlinearities involved in drop test, simple shock tests are performed on subsystems prior to conducting drop test on the full product and finite element modeling and calibration processes are carried out simultaneously. In addition to simple shock tests, PWB and plastic housing natural frequencies are found experimentally. Finding the natural frequencies plays an important role in approximating the material properties and extracting the damping ratios to a good accuracy. Modulus of elasticity can be estimated by matching the frequencies of the FEA model with that of experiment. In addition, the damping parameters can be obtained by calibrating the acceleration amplitudes of the frequency response function of the FEA model with respect to experimental data. Shock tests are performed according to MIL-STD-810E [17]. Furthermore, in this research, product level drop testing is conducted, similar to that recommended in JEDEC JESD22-B104-B standard [18]. According to the JEDEC JESD22-B104-B standard, the product is attached to a drop table at a certain orientation, raised to the desired height, and subsequently the drop table is released. Unlike in JEDEC JESD22-B104-B, in this study, the product does not detach from the drop table seconds before the impact for a free drop and additional impacts due to bounce after the initial impact. Since the mission of this research is to calibrate the finite element models with experimental data, rather than to study product reliability, the product remains attached to the drop table during the entire drop event and experiences only the initial impact, for purposes of simplicity. 


\section{Chapter 2 Shock and Dynamic Loading in Portable Electronics}

The text of this chapter is taken from a paper that has been submitted for publication. This paper is the first portion of a two-part study and contains the results of experiments that were conducted to aid in the development of a systematic approach for modeling the dynamic response of complex portable electronic devices subjected to shock and drop loading conditions. The purpose of the computational models is to enable early design iterations and minimize costly trial-and-error empirical design-fixes later during qualification testing or after the product design has been finalized. In this chapter, the product under study and its subassemblies are subjected to various shock and drop loading to quantify their response to various operational and environmental dynamic loads. In the second part of the study, which is presented in Chapter 3, the experimental results obtained in this chapter will be used to guide and calibrate the finite element models for each phase of the tests. In this chapter, random vibration and shock test results are presented first and followed by drop test results. 


\title{
SHOCK AND DYNAMIC LOADING IN PORTABLE ELECTRONIC ASSEMBLIES: \\ PART I- EXPERIMENTAL RESULTS
}

\author{
Alex F. Askari Farahani, Moustafa Al-Bassyiouni, Abhijit Dasgupta (Corresponding \\ Author) \\ CALCE Electronic Products and Systems Center \\ Mechanical Engineering Department \\ University of Maryland, College Park, MD 20742 USA \\ Sheldon Tolchinsky, Jack Crystal \\ Arbitron INC. \\ 9705 Patuxent Woods Drive, Columbia, Maryland 21046
}

\section{Abstract:}

The development of portable electronics poses design challenges when evolving new designs for high strain-rate life-cycle loading, such as in drop events, blast events, vibration, ultrasonic process steps, etc. This paper is the first part of a two part study and discusses an experimental investigation of the transient response of a portable electronic product and its subassemblies, to dynamic mechanical loading encountered in drop and shock conditions. The portable electronic product tested in this study consists of a circuit card assembly and a battery pack supported in a twopiece plastic housing with a separate battery compartment. Dynamic loading, consisting of various shock profiles, is applied using an electrodynamic shaker. A number of drop tests are also conducted on a drop tower. Fourier Transform technique (FFT) is utilized to analyze the dynamic response of the PWB and the plastic housing in the frequency domain. These results will be used in the second part of the study to investigate the ability of finite element models to accurately capture this transient response of complex portable electronic assemblies under shock and drop loading. 


\subsection{Introduction:}

Portable electronic devices are frequently subjected to unintentional drops and shock during transportation, handling and usage. Drop reliability of electronic assemblies in the product depends to a great degree on the response of the printed wiring boards (PWBs) in the assembly. Therefore, it is of great interest to fully understand how PWBs behave inside portable electronic devices when subjected to dynamic loading, such as that in drop loading. Various factors, such as drop height, mass of the product, and drop orientation can significantly influence the amount of energy transmitted to the PWB. A systematic investigation is required to identify the energy paths through which dynamic forces are transmitted to the PWBs inside electronic products. In industry, board-level shock tests are often used to study the reliability of interconnections between PWBs and components on the board, under conditions similar to those experienced during usage [1]. Seah [1] conducted a comprehensive study on several portable electronic devices to discuss differences between board-level and product-level tests and methods to develop a realistic boardlevel test that can represent the actual conditions encountered during usage. Seah [1] concluded that board response significantly changes with board size and casing design. Tan [2] performed product-level drop tests to study the impact performance of portable electronic devices, and concluded that impact force magnitudes are highly dependent on drop orientation. In general, for angled orientations, a moment arm is created between the impact point and the center of mass of the product causing the object to rotate and not absorb the full energy of the impact [2]. Horizontal and vertical orientations are, however, subjected to a more direct impact and fewer 
rotations, which results in higher internal board accelerations and strains. According to Tan [2] the horizontal drop orientation generates the highest bending strains in PWBs. Thus, in this study, all the testing is performed in the horizontal orientation. Similarly, Lim [3] utilized a controlled orientation drop tester and a high speed camera to study the impact behavior of portable electronic devices. Lim [3] realized that it is very hard to interpret PWB acceleration profiles, because of the complex interactions between the PWB and the housing. It is very likely for the board to impact against the housing and experience large accelerations [3]. Luan [4] thoroughly investigated the relationship among drop height, pulse duration, and acceleration levels achieved during drop tests. He also discussed how different test variables such as number of mounting screws as well the tightness of the screws could potentially affect the repeatability of the test results. Pekka [5] presented a method in which drop testing is replaced by vibration testing to study the mechanical durability of electronic components under shock loading conditions. In drop tests, the bending of boards is the foremost deriver of failure in surface mount components [5]. Pekka suggested that the same failure mechanisms can be generated in vibration tests, providing the amplitude and frequency of vibration tests to be similar to that of drop tests. This is particularly very useful since vibration test systems are more flexible and can be performed in conjunction with other tests such as thermal cycling [5].

This paper is the first part of a two-series study. This part of the study utilizes a combination of simple shock, random vibration, and drop tests to study the dynamic response of a portable electronic assembly and its main components such as printed wiring board, spring contacts, and plastic housing. In the second part of the study the 
focus will be on developing finite element models for the system under study. Simulation results will be compared with the experimental results of this paper, to investigate whether or not FEA models are capable of accurately predicting the drop response of such highly non-linear complex assemblies.

\section{$\underline{2.2 \text { Design of Experiment }}$}

Section 2.2 describes various features of the device under study. Additionally, test approach and matrix are detailed in this section.

\subsubsection{Test Specimen}

The device in this study consists of the following parts:

- Main PWB. The heaviest and most compliant sub-assemblies on this PWB are:

a. Three LEDs in a housing

b. A microphone and its housing

c. Two Au-plated leaf springs connecting the PWB to a pair of charge pins

- Metal charge contact pins (2 pins, one on each side)

- Top and bottom housing

- Battery and battery case

The top and bottom housings, as well as the battery case, are made of Lexan 500. The PWB material is FR-4. The PWB is seated on several ribs that are located along the walls of the top and bottom housings. It is also seated on the battery case, which contains a rectangular shaped battery. The PWB is also held in place by between two 
star-shaped tapered posts (one fixed on the bottom housing and the other fixed on the battery case) and two corresponding caps fixed on the top housing (Refer to Figure 2-1 and Figure 2-2).
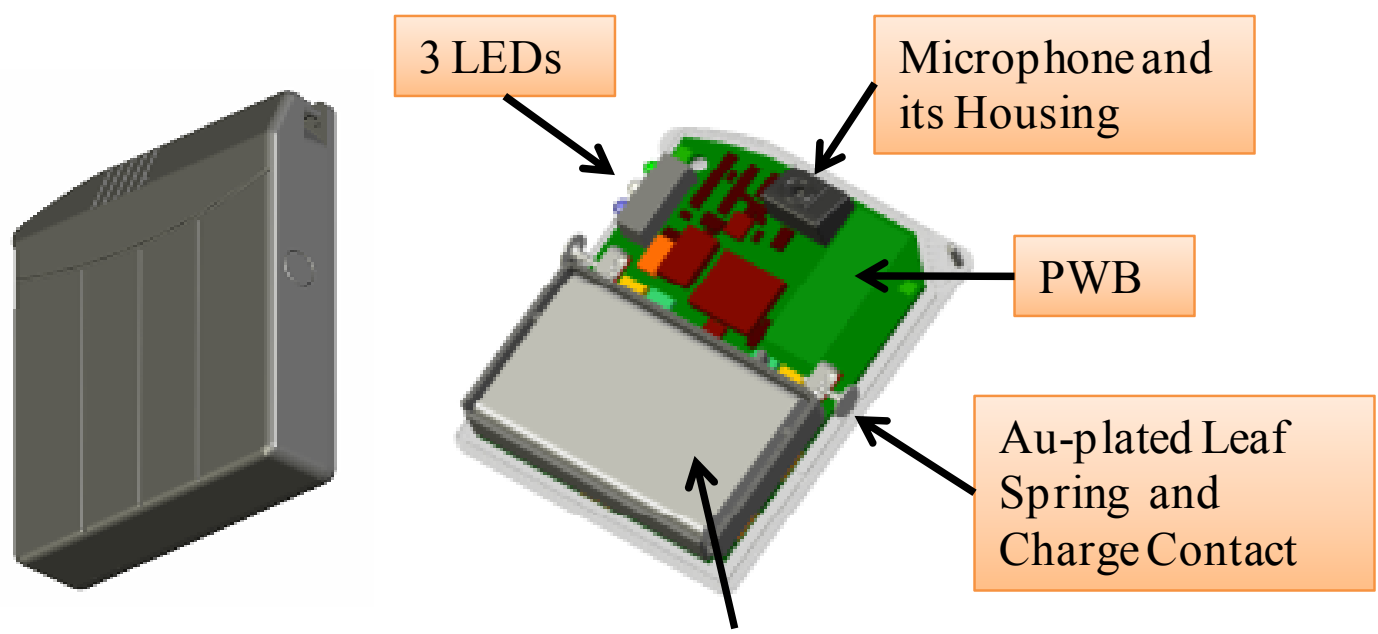

\section{Battery and Battery Case}

Figure 2-1 Device elements

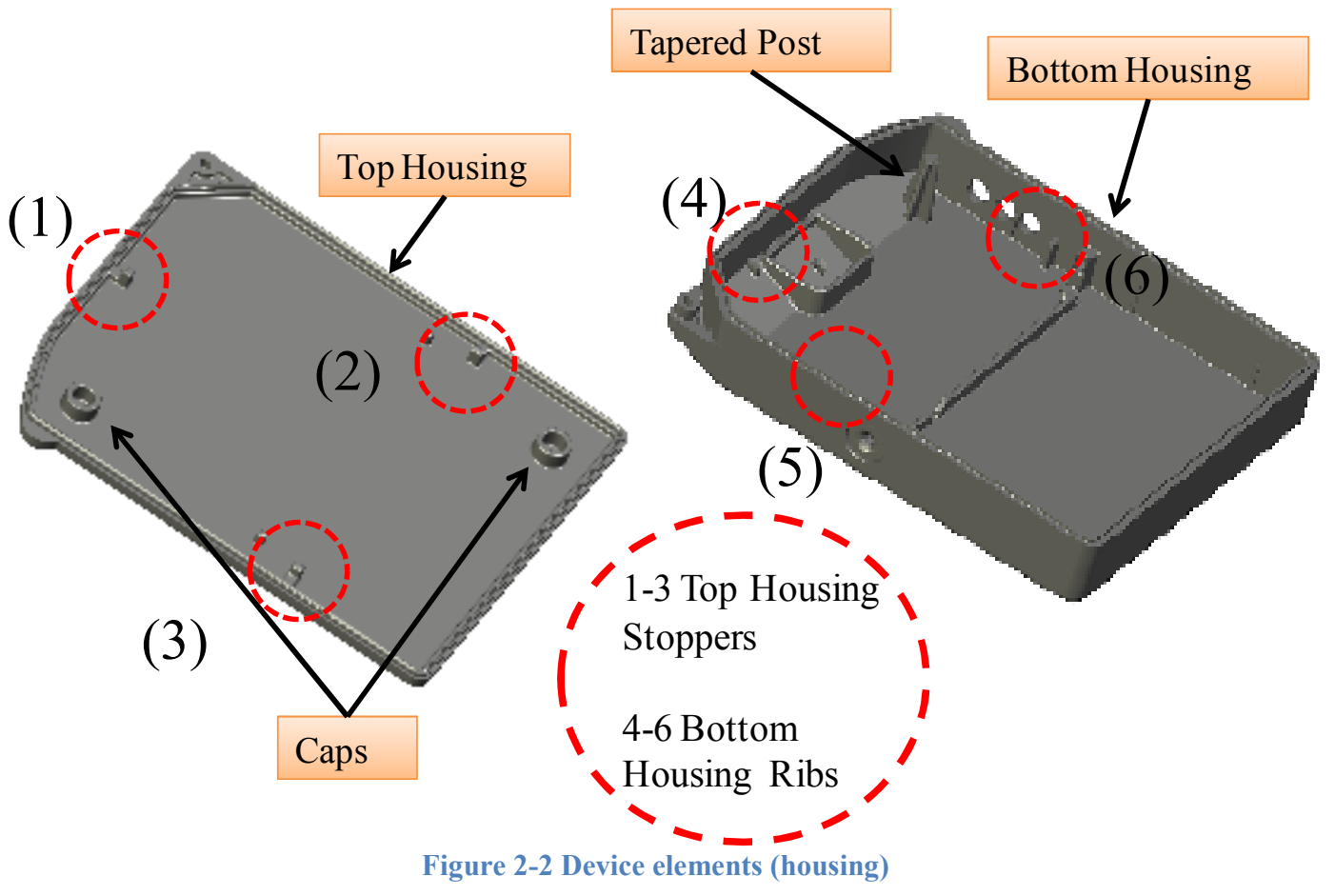


The two charge contact pins are connected to the PWB through Au-plated leaf springs, which are soldered to the main PWB, as shown in Figure 2-3.

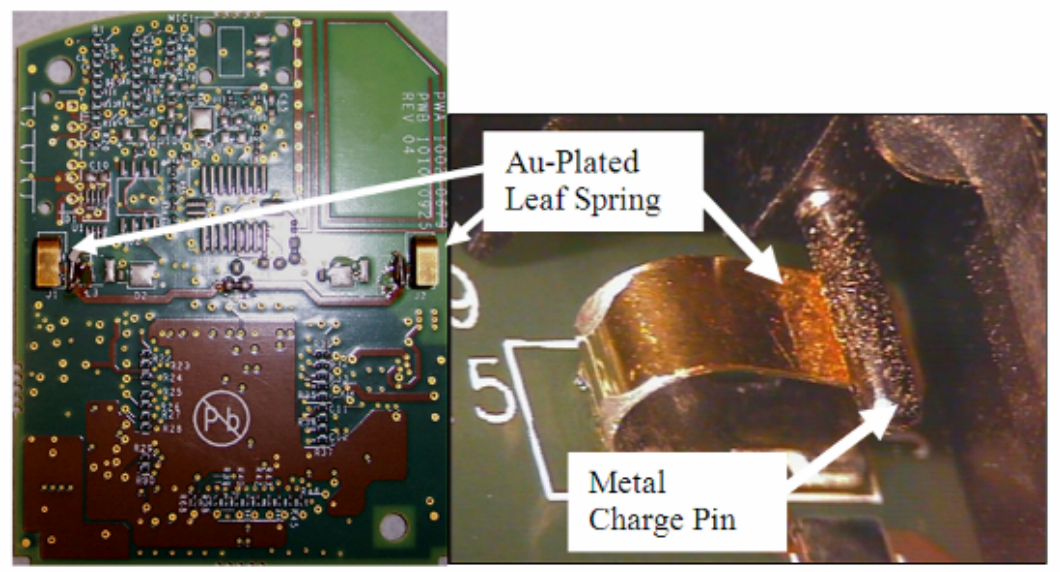

Figure 2-3 Au-plated leaf springs

\subsubsection{Approach and Test Matrix}

The challenge in system-level modeling of drop loading is that discrepancies between simulation and experimental results are difficult to trace back to the source of the problem. Therefore, troubleshooting is very difficult. To overcome this problem, a systematic building-block approach is used in this study, where the full complex system is divided into simpler constituent subsystems as shown in Figure 2-4. To eliminate some of the nonlinearities involved in drop test of complex assemblies, broad band and simple shock tests are performed on the subsystems prior to conducting drop test on the full product. The results of the sub-system tests are used to guide and calibrate finite element models of the test specimen (the simulation results are presented later in Part II of this study). Finding the natural frequencies of the PWB and the housing plays an important role in approximating the material properties and extracting the damping ratios with good accuracy. 


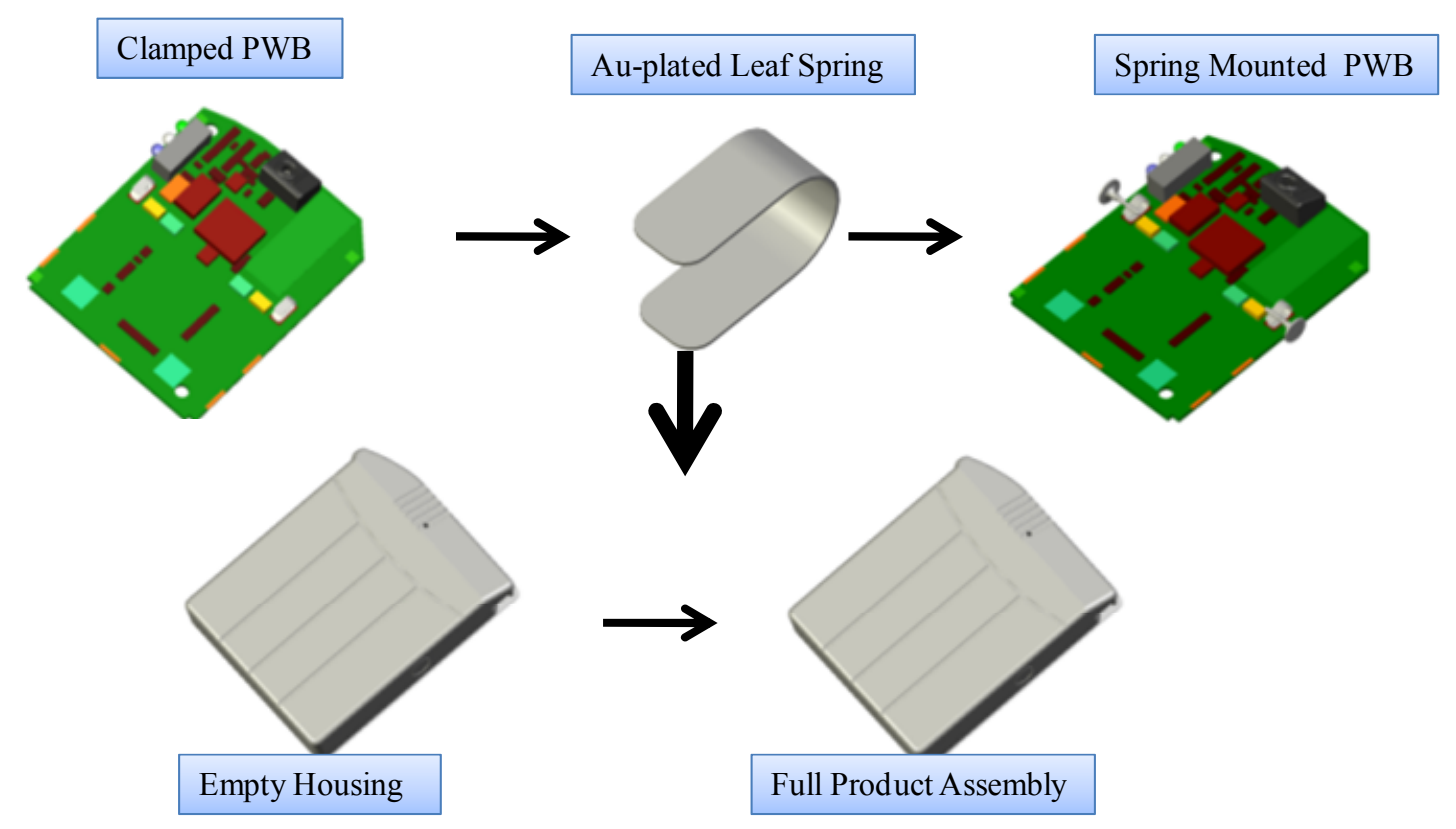

Figure 2-4 Test Approach

To ensure repeatability, each test is conducted several times. Acceleration levels and the test matrix are detailed in Figure 2-5. As shown in Figure 2-5 shock tests are performed on the bare PWB with clamped boundary conditions, as well as the full product assembly, at various acceleration levels, to investigate the effects of acceleration level on the dynamic response. Similarly, drop tests are also performed on clamped PWB and on the full assembly at different acceleration levels. The drop test matrix is also detailed in Figure 2-5.

\begin{tabular}{|c|c|c|c|c|c|c|}
\hline & Broad Band Test & & \multicolumn{3}{|c|}{ Half-sine Shock Test } & \multirow{2}{*}{$\frac{\text { Drop Test }}{1600 \mathrm{G}}$} \\
\hline \multirow{2}{*}{ Clamped PWB } & \multirow{2}{*}{$60-800 \mathrm{~Hz}$ at $.01 \mathrm{~g}^{2} / \mathrm{Hz}$} & Acceleration & $17 \mathrm{G}$ & $35 \mathrm{G}$ & $63 \mathrm{G}$ & \\
\hline & & No. of Trials & 10 & 10 & 2 & 10 \\
\hline $\begin{array}{c}\text { Spring Supported } \\
\text { PWB } \\
\end{array}$ & $60-800 \mathrm{~Hz}$ at $.01 \mathrm{~g}^{2} / \mathrm{Hz}$ & & & & & \\
\hline Empty Housing & $50-3200 \mathrm{~Hz}$ at $.01 \mathrm{~g}^{2} / \mathrm{Hz}$ & & & & & \\
\hline \multirow{2}{*}{ Full Product } & \multirow{2}{*}{$50-3200 \mathrm{~Hz}$ at $.01 \mathrm{~g}^{2} / \mathrm{Hz}$} & Acceleration & \multicolumn{3}{|c|}{$30 \mathrm{G}$} & $1500 \mathrm{G}$ \\
\hline & & No. of Trials & \multicolumn{3}{|c|}{10} & 5 \\
\hline
\end{tabular}

Figure 2-5 Test matrix 


\section{$\underline{2.3 \text { Shock Test Set Up }}$}

Section 2.3 discusses the test set up for the various shock tests that are detailed in Figure 2-5. The test results are discussed in section 2.5.

\subsubsection{Test Set Up for Broad Band and Shock Loading of Clamped PWB}

An electro dynamic shaker, shown in Figure 2-6, is used to perform a halfsine classical shock test on the specimen. As shown in the test matrix the shock test is performed at three $\mathrm{G}$ levels, 17G, 35G and 63G. Pulse duration is fixed at $6 \mathrm{~ms}$, according to the MIL-STD-810 standard. In addition to the shock test, a broad band

test with $0.01 \mathrm{~g}^{2} / \mathrm{Hz}$ PSD level over a frequency range of $60-800 \mathrm{~Hz}$ is conducted to find the natural frequencies of the test article. 


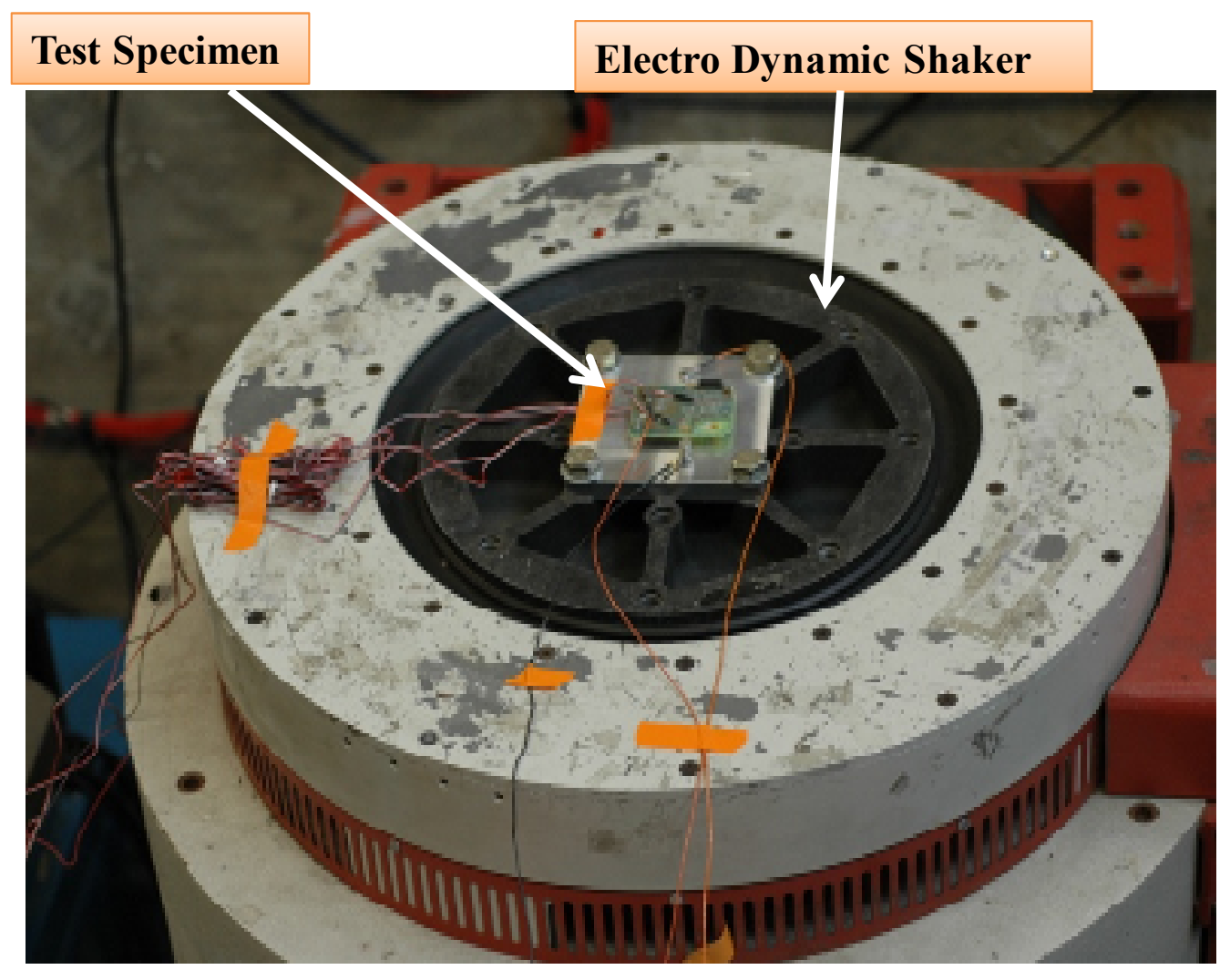

Figure 2-6 Electro dynamic shaker with test specimen

In both board-level and product-level shock and drop tests, the location of monitoring instrumentation requires careful attention. The Printed Wiring Assemblies (PWAs) are allowed to flex during these tests, resulting in non-uniform distributions of flexural strain and acceleration throughout the PWB. The distribution depends on how the PWB is mounted on the fixture. Figure 2-7 demonstrates how the bare PWB is mounted on the shaker in the shock test conducted in this study. The PWB sits on two spacers and is fixed at points 1 and 2 with nuts and washers. The accelerometer on top, Accelerometer $\mathrm{C}$, controls the shaker excitation. Acceleration at the free end of the board is expected to be higher than at other locations on the board. Thus, an accelerometer (Accelerometer B) is placed at this location. Moreover, to monitor the 
dynamic flexural strain of the test PWB, four strain gages are placed on the PWB. Two of the strain gages can be seen in Figure 2-7 (Labeled with red dotted circles); the rest of them are on the other side of the board, behind the Au-plated leaf springs. Considerable amount of flexural strain is expected at point one since it is fixed. Strain level, however, at other locations is expected to be small.

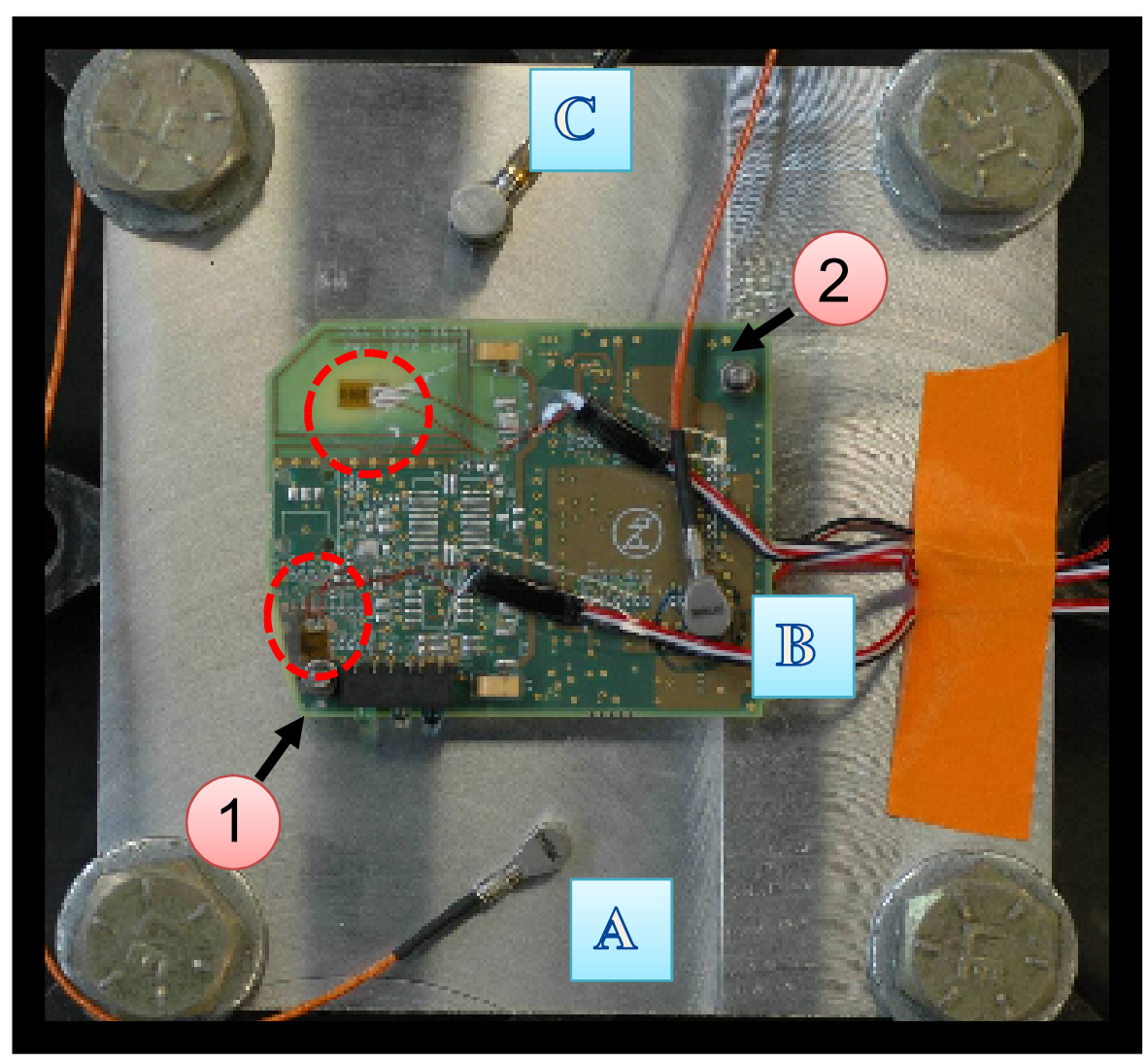

Figure 2-7 Bare board shock test set up. The PWB is fixed at points 1 and 2. Accelerometers A and B measure the fixture and PWB response. Accelerometer $\mathrm{C}$ controls the shaker excitation. Strain gages are labeled with red dotted circles.

The accelerometer on the bottom (Accelerometer A) measures the acceleration of the fixture. The output of this accelerometer will be used as an input pulse in the finite element modeling of bare PWB, reported later in Part II of this 
study. Data acquisition sampling rate for simple shock test is set at 5000 samples per second. Furthermore, a $10 \mathrm{KHz}$ low pass filter is used. A low pass filter provides a smoother signal by removing the high-frequency noise, leaving only the long-term trend. Before running the experiment, all the accelerometers were calibrated to ensure accurate readings. Two recently calibrated Dytran accelerometers (accelerometers A\&B in Figure 2-7) are calibrated with respect to each other. Figure 2-8 shows that there is at most, a $1.0 \%$ difference between these two accelerometers.

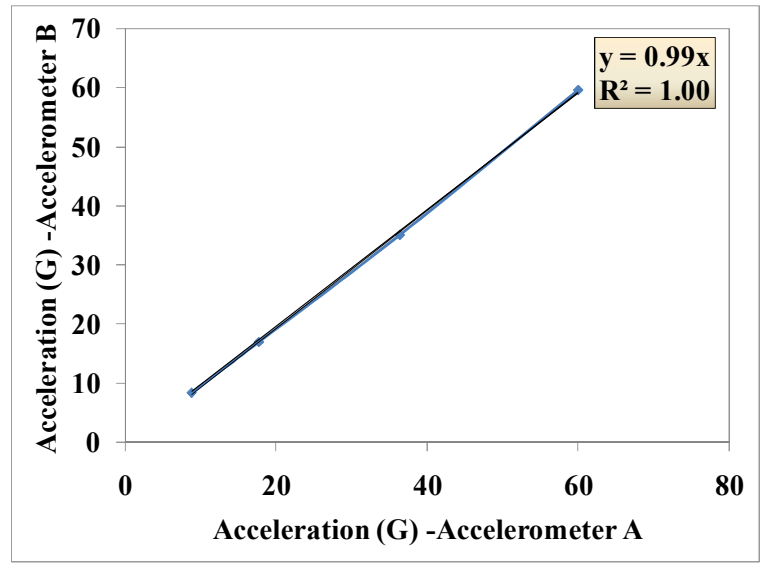

Figure 2-8 Accelerometer calibration

Accelerometer $\mathrm{C}$ was also calibrated with respect to the Dytran accelerometers. Figure 2-9 indicates that there is a $12 \%$ difference that will be taken into account throughout in the experiment. 


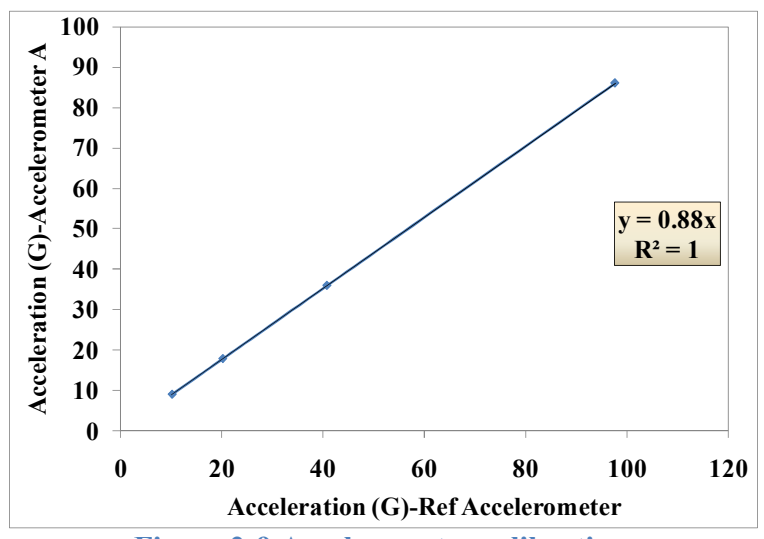

Figure 2-9 Accelerometer calibration

Figure 2-10 illustrates a schematic of the bare PWB test set up and how all the elements of the test are connected to each other. Data acquisition configuration and test set up for spring-loaded PWB and for the full assembly remain the same as that of the bare PWB. The only difference among these tests is the type of the fixture that is being used. Therefore, in the subsequent sections, only the fixtures for shock testing of the spring-loaded PWB and of the full assembly are discussed.

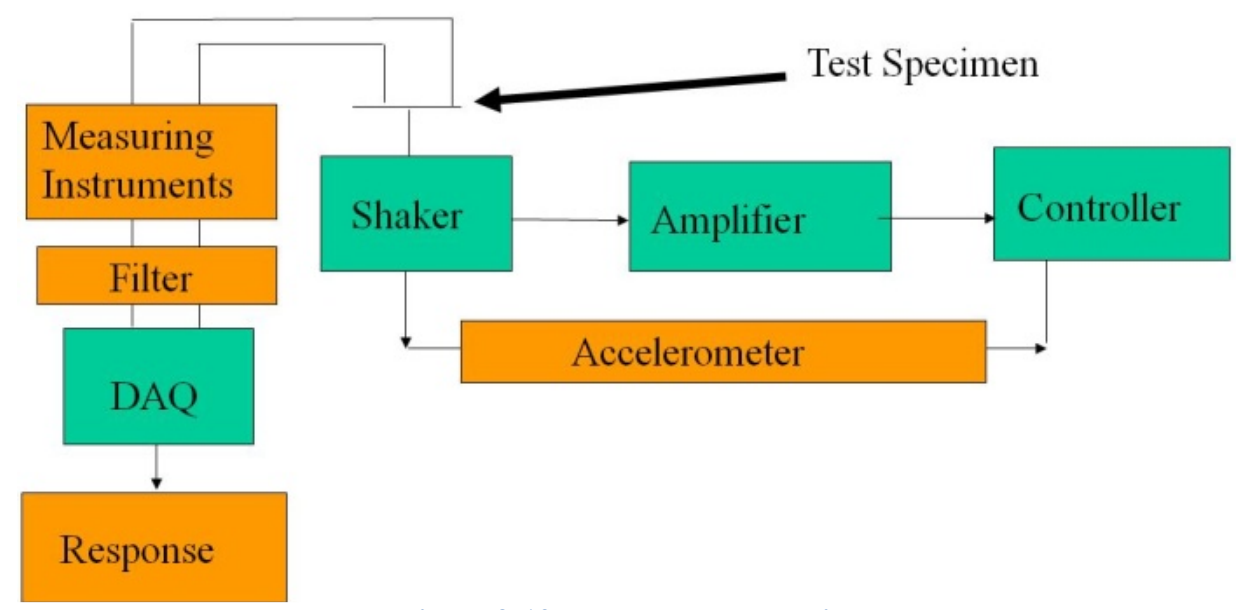

Figure 2-10 Test set up schematic

\subsubsection{Test Set Up for Broad Band Excitation of Spring-mounted PWB}

The test fixture for the spring-loaded PWB is illustrated in Figure 2-11. Similar to the test set up for shock loading of the clamped PWB, the spring loaded 
PWB is fixed at points 1 and 2 with nuts and washers. Additionally, as shown in Figure 2-3, there are two charge contact pins that are connected to the PWB through the Au-plated leaf springs in the actual product. Therefore, to roughly simulate the constraints in the actual product, two metal pins added to the fixture to push down on the Au-plated leaf springs. The location of the accelerometers and strain gages are determined in a similar fashion to that of bare PWB. Accelerometers A and B measure the fixture and PWB excitations respectively. Furthermore, accelerometer C controls shaker excitation.

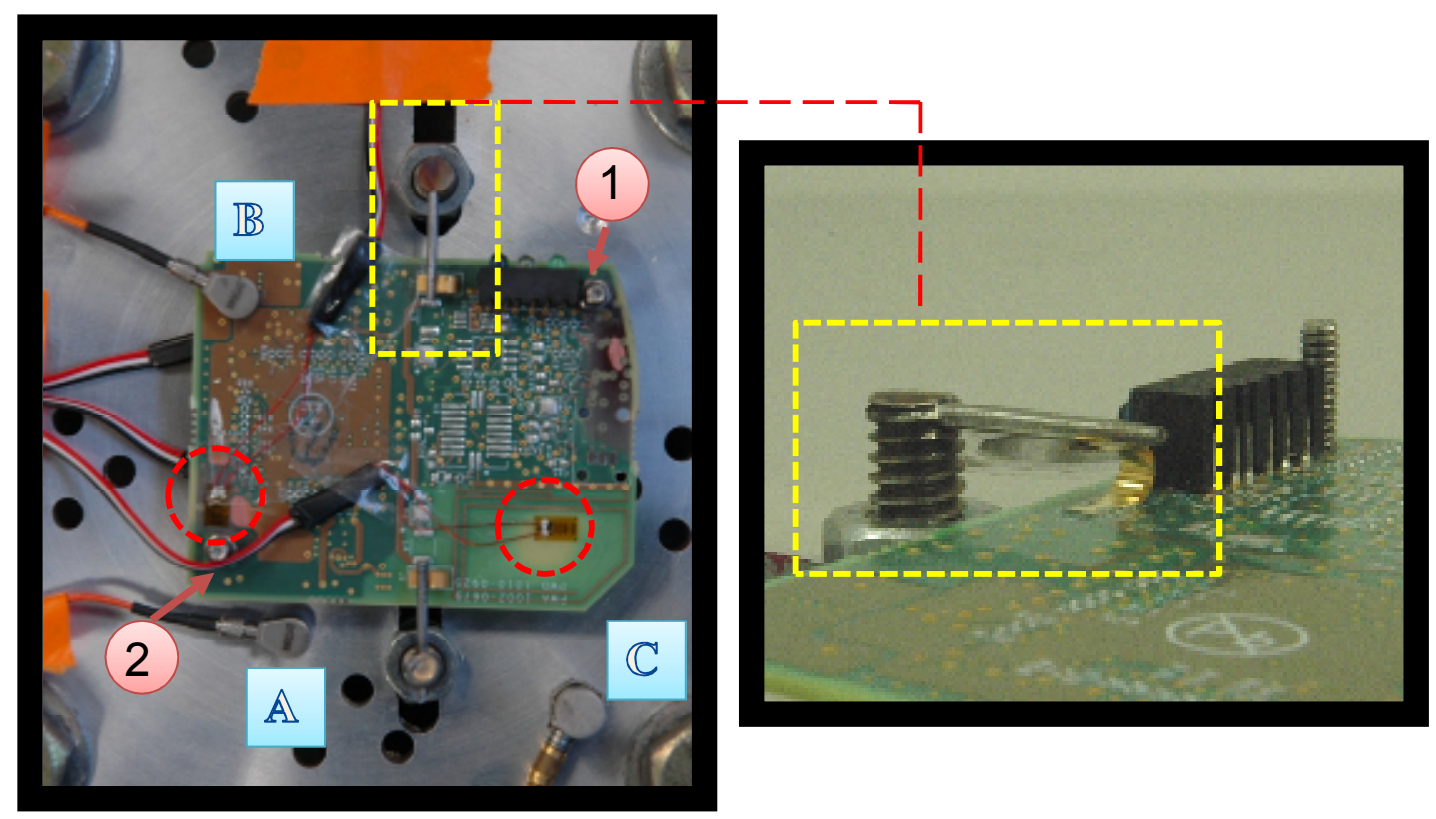

Figure 2-11 Spring-mounted board test set up- The PWB is fixed at points 1 and 2. Accelerometers A and B measure the fixture and $\mathrm{PWB}$ response. Accelerometer $\mathrm{C}$ controls the shaker excitation. Strain gages are labeled with red dotted circles.

\subsubsection{Test Set Up for Broad Band Excitation of Plastic Housing}

As mentioned in the preceding sections, finding the natural frequencies of the empty case is very useful for the tasks reported in Part II of this study, viz. to calibrate its damping properties and to verify the accuracy of the finite element 
model. Figure 2-12 demonstrates the fixture and instrumentation for the tests conducted on the empty plastic housing.

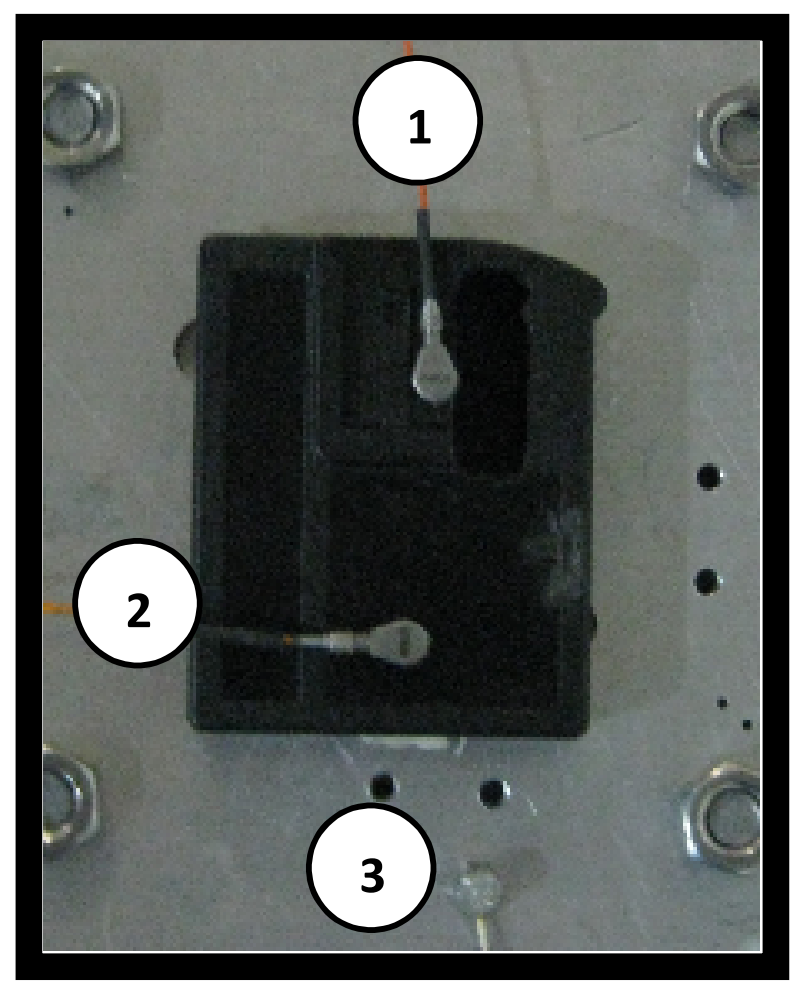

Figure 2-12 Test set up for broad band test on housing. Accelerometers 1 and 2 monitor the response of empty plastic housing. Accelerometer 3 controls the shaker excitation.

The empty plastic housing is placed flat on the fixture and the bottom surface is attached to the fixture with double sided tape. Accelerometers 1 and 2 monitor the response of the empty plastic housing. Since the plastic housing is attached to the fixture with double sided tape, it is possible for the double sided tape to provide only partial but not complete constraint on the bottom housing. This could cause the bottom housing to deform and consequently modify the natural resonant modes. According to Figure 2-13 and Figure 2-14, preliminary FEA analysis predicts that the second mode shape is quite sensitive to the boundary conditions and changes significantly as the boundary condition on the bottom surface is changed from 
completely fixed to partially constrained. In the partially constrained case, the bottom housing is only fixed along its edges.

As shown in Figure 2-14, for the partially constrained case, the bottom housing deforms rather than the top housing in the second mode. Therefore, it is essential to ensure that the correct natural modes are compared against simulation results. This is accomplished by placing two accelerometers at locations 1 and 2 shown in Figure 2-12 on the empty plastic housing, and monitoring their phase responses, as discussed later in Section 2.5.1.4.

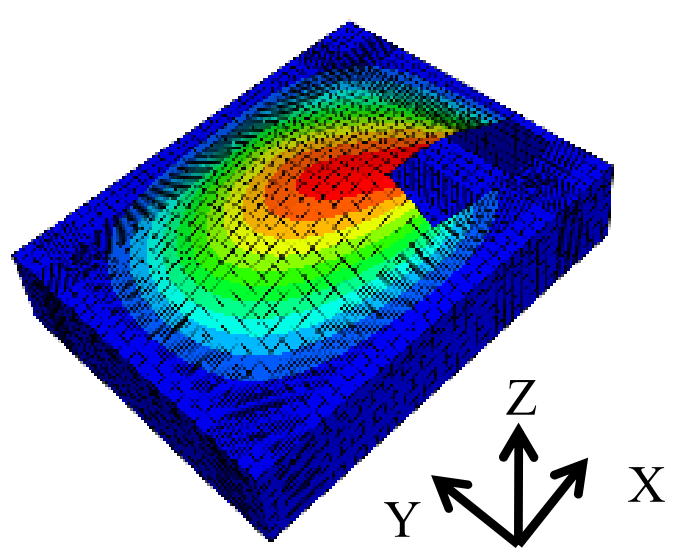

MODE 1

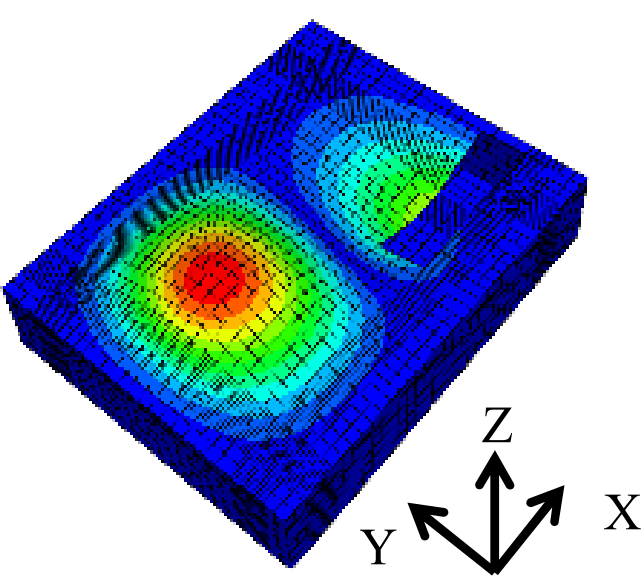

MODE 2

Figure 2-13 First two natural modes of the empty housing when the bottom of the housing is completely constrained. 

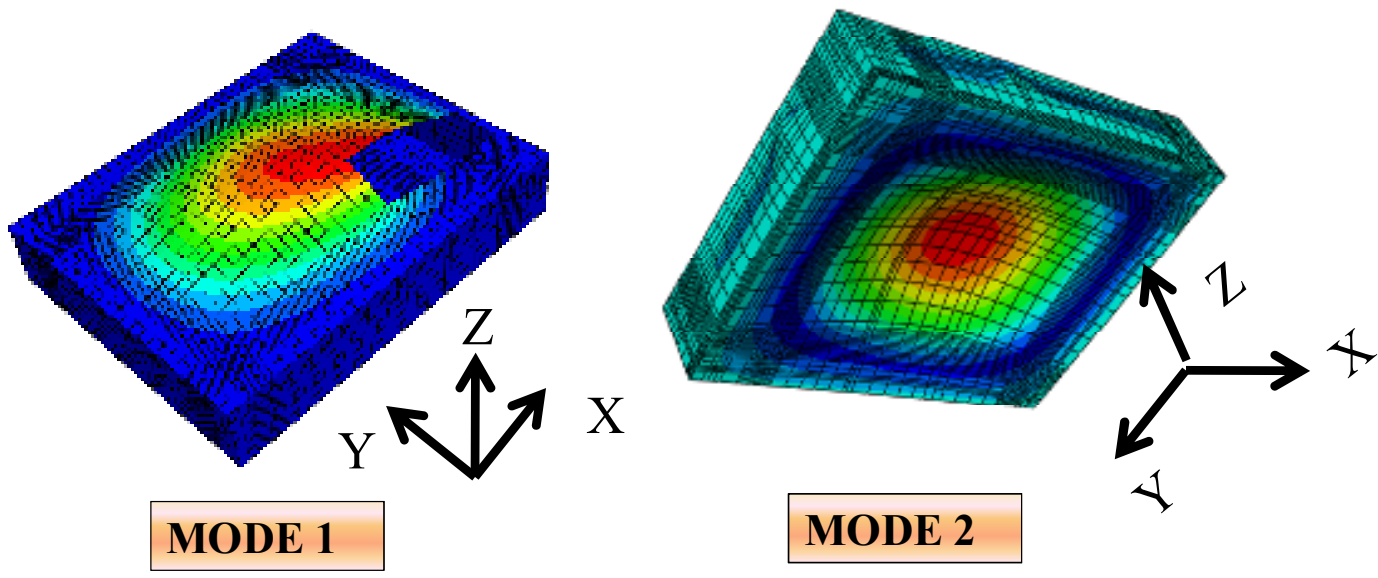

Figure 2-14 First two natural modes of the empty housing when the bottom of the housing is partially constrained.

Furthermore, Accelerometer 3 measures the fixture acceleration. The frequency range for the random vibration test is set to $50 \mathrm{~Hz}-3200 \mathrm{~Hz}$ at $0.01 \mathrm{~g}^{2} / \mathrm{Hz}$. The sampling rate for data acquisition is 8192 samples per second.

\subsubsection{Test Set Up for Broad Band Excitation of Complete Product Assembly}

The test fixture for broad band testing of the complete product assembly is shown in Figure 2-17. The product is laid down face up, flat on the fixture and clamped down at three points. As shown in Figure 2-18, small, thin aluminum plates are positioned underneath the clamps to distribute the load during the test. Part of the top housing is removed to accommodate the accelerometer (Accelerometer III) on the PWB. Accelerometer I and II measure the response of the fixture and the plastic housing, respectively. To ensure that the natural frequencies of the clamping fingers do not coincide with that of the full product, it is essential to obtain the natural frequencies of the clamping fingers prior to performing the broad band test on the full product assembly. Therefore, in a separate test an accelerometer is placed on the clamping fingers of the fixture as shown in Figure 2-15. The accelerometer in Figure 
2-15 measures the response of the clamping fingers to the broad band excitation. The frequency range for the random vibration test is set to $50 \mathrm{~Hz}-3200 \mathrm{~Hz}$ at $0.01 \mathrm{~g}^{2} / \mathrm{Hz}$. The sampling rate for data acquisition is 8192 samples per second.

Furthermore, in order to analyze the broad band test results of the full product, it is necessary to understand how the plastic housing and the PWB behave individually under the boundary conditions of the full product. This understanding will also distinguish among the natural modes of the system driven by the plastic housing and the PWB. As a result, the empty plastic housing, described in Section 2.3.4, is clamped down onto the fixture described earlier in this section for the broad band test of the full product. Subsequently, the same broad band test is performed on the clamped empty housing to find its natural frequencies. The fixture for the broad band test of the empty plastic housing is shown in Figure 2-16. Accelerometer $\mathrm{H}$ is placed on the same location on the plastic housing as the accelerometer II shown in Figure 2-17 and measures the response of the plastic housing. Accelerometer F measures the response of the fixture. 


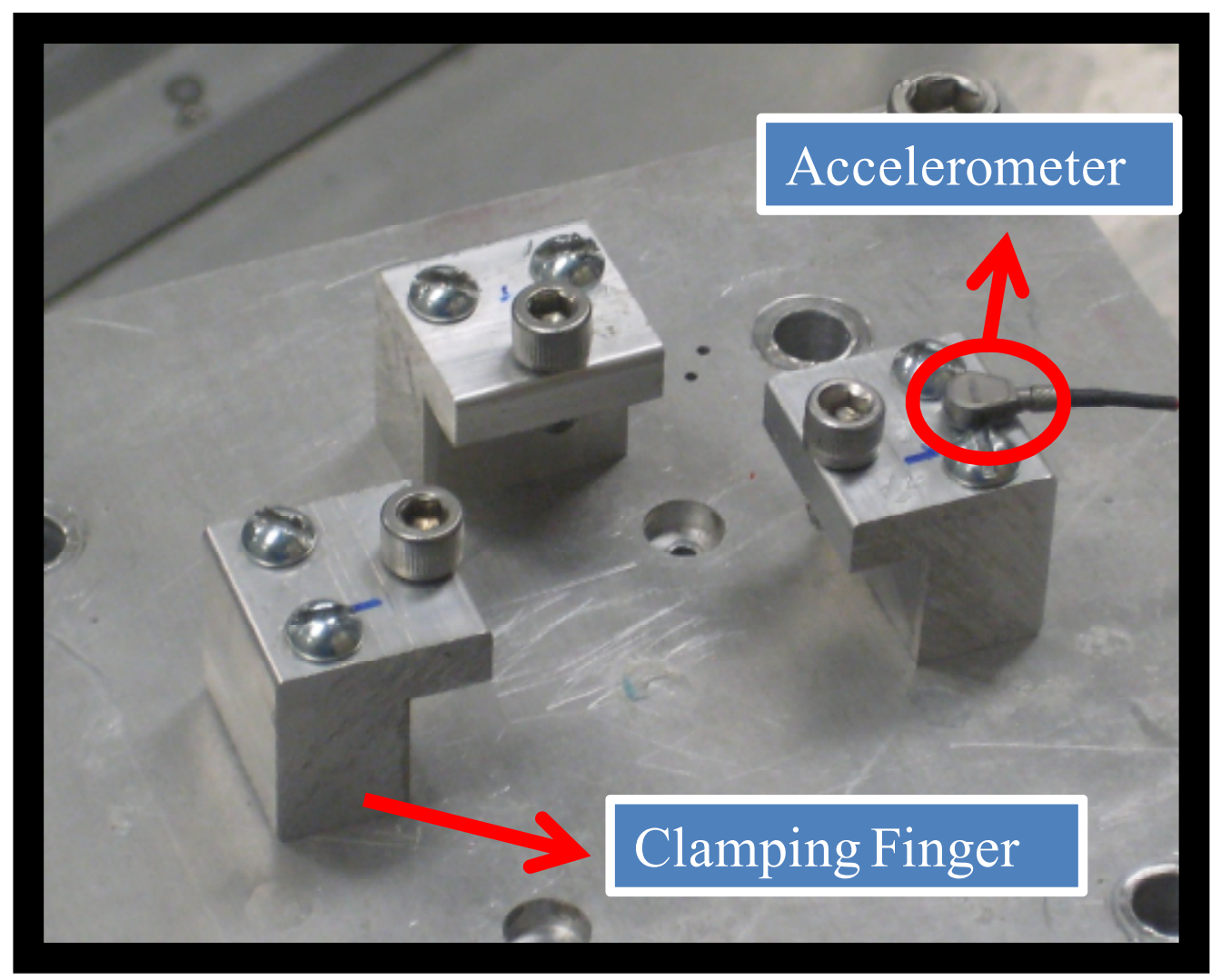

Figure 2-15 Test set up for broad band excitation of the full product and plastic empty housing. The figure illustrates the clamping mechanism used to fix the product to the fixture. The Accelerometer $r$ measures the response of the clamping finger to ensure its natural frequencies do not coincide with those of the full product. 


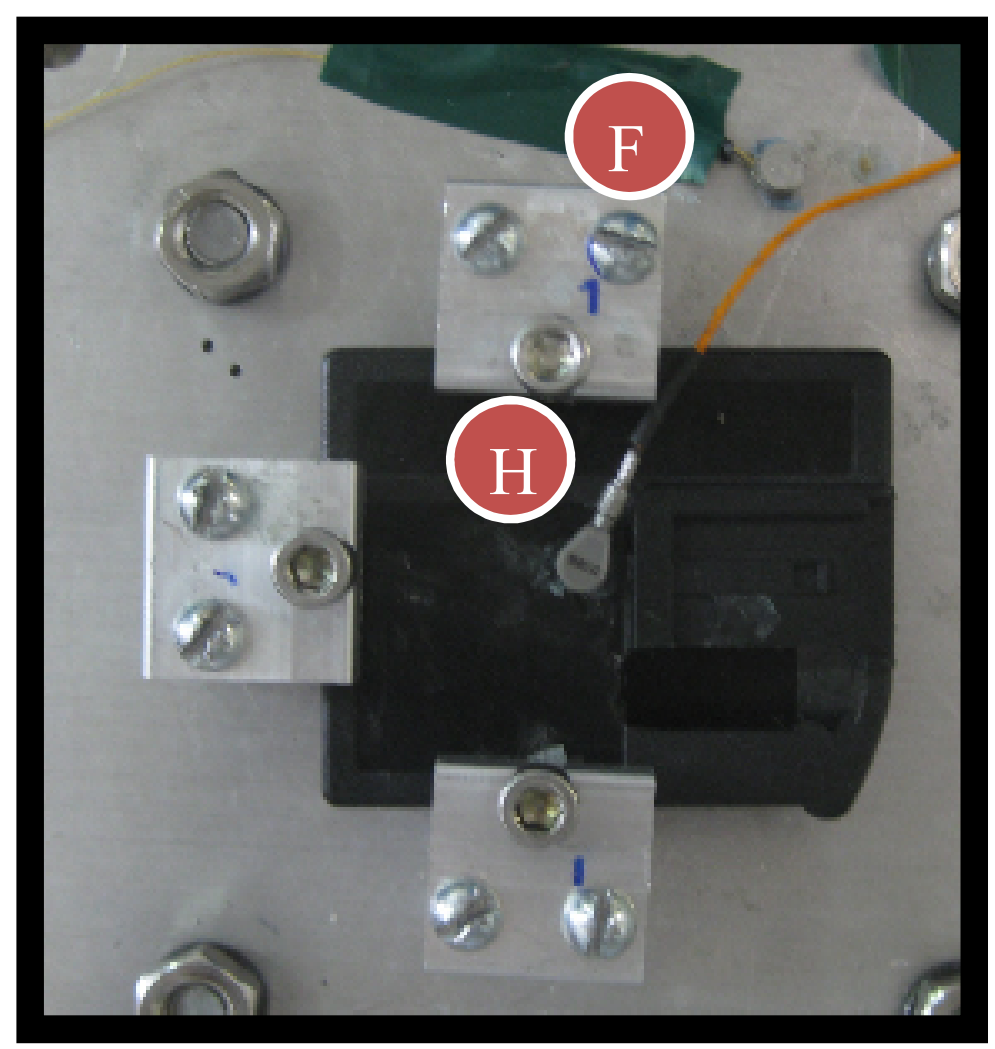

Figure 2-16 Test set up for the broad band test on empty plastic housing. Accelerometer $\mathbb{H}$ is placed on the same location as the accelerometer II shown in Figure 2-17 and measures the response of the plastic housing. Accelerometer $\mathrm{F}$ measures the response of the fixture.

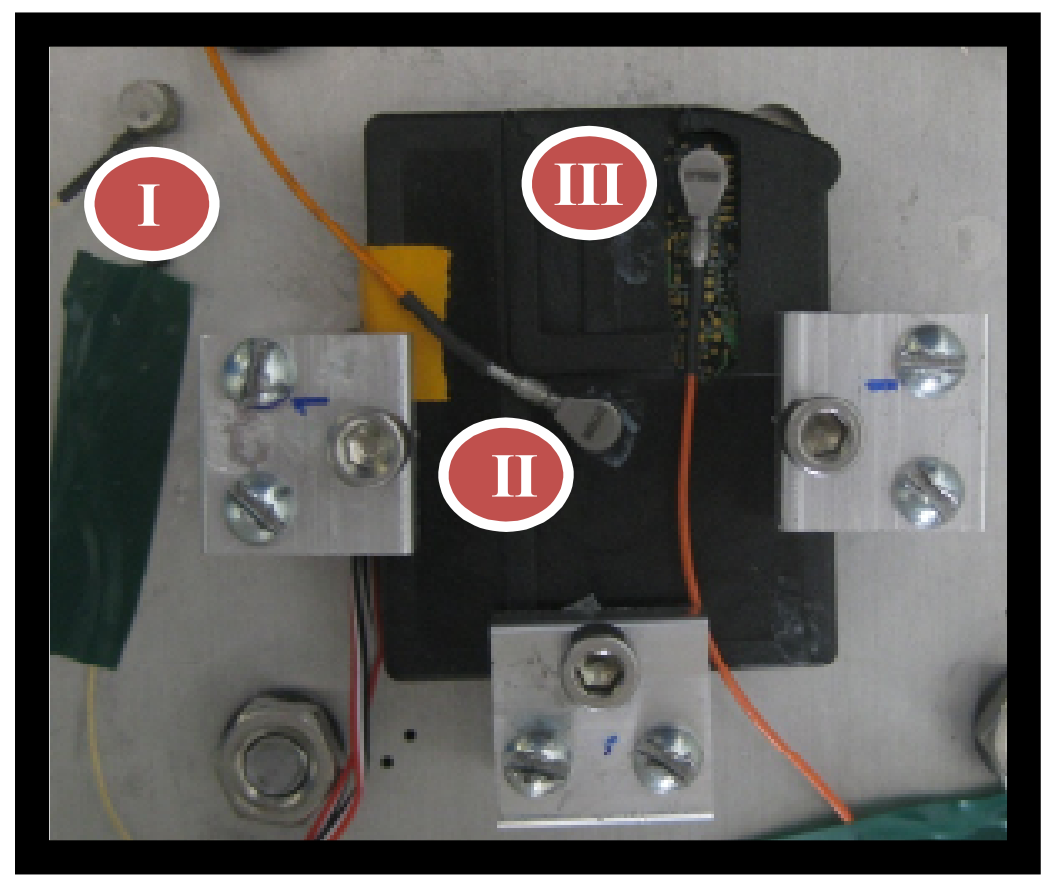

Figure 2-17 Test set up for broad band test on full product assembly. Accelerometer I monitors the fixture response. Accelerometer II and III measure the plastic housing and PWB response. 


\subsubsection{Shock Test Up of Complete Product Assembly}

The test fixture for shock testing of the complete product assembly is shown in Figure 2-18. The fixture of the full product shock test is very similar to that of shown in Figure 2-17 described in section 2.3.5. The accelerometer locations are, however, slightly different. The differences are explained in this section. Accelerometer B is located near the LED location, because of the possibility of high accelerations at this region due to the potential for impact between the LEDs and the side walls of the product housing. Two strain gages are mounted on the PWB at Points 1 and 2 to monitor the board response. The strain is measured in the $\mathrm{x}$ direction as shown in Figure 2-18. A hole is drilled on the side of the bottom housing to route the strain gage wires out of the product. Shock test on the full product is only executed at one acceleration level of $30 \mathrm{G}$. Similar to the bare PWB shock test, the pulse duration is fixed at $6 \mathrm{~ms}$. Furthermore, Accelerometer "A" measures the response of the fixture. 


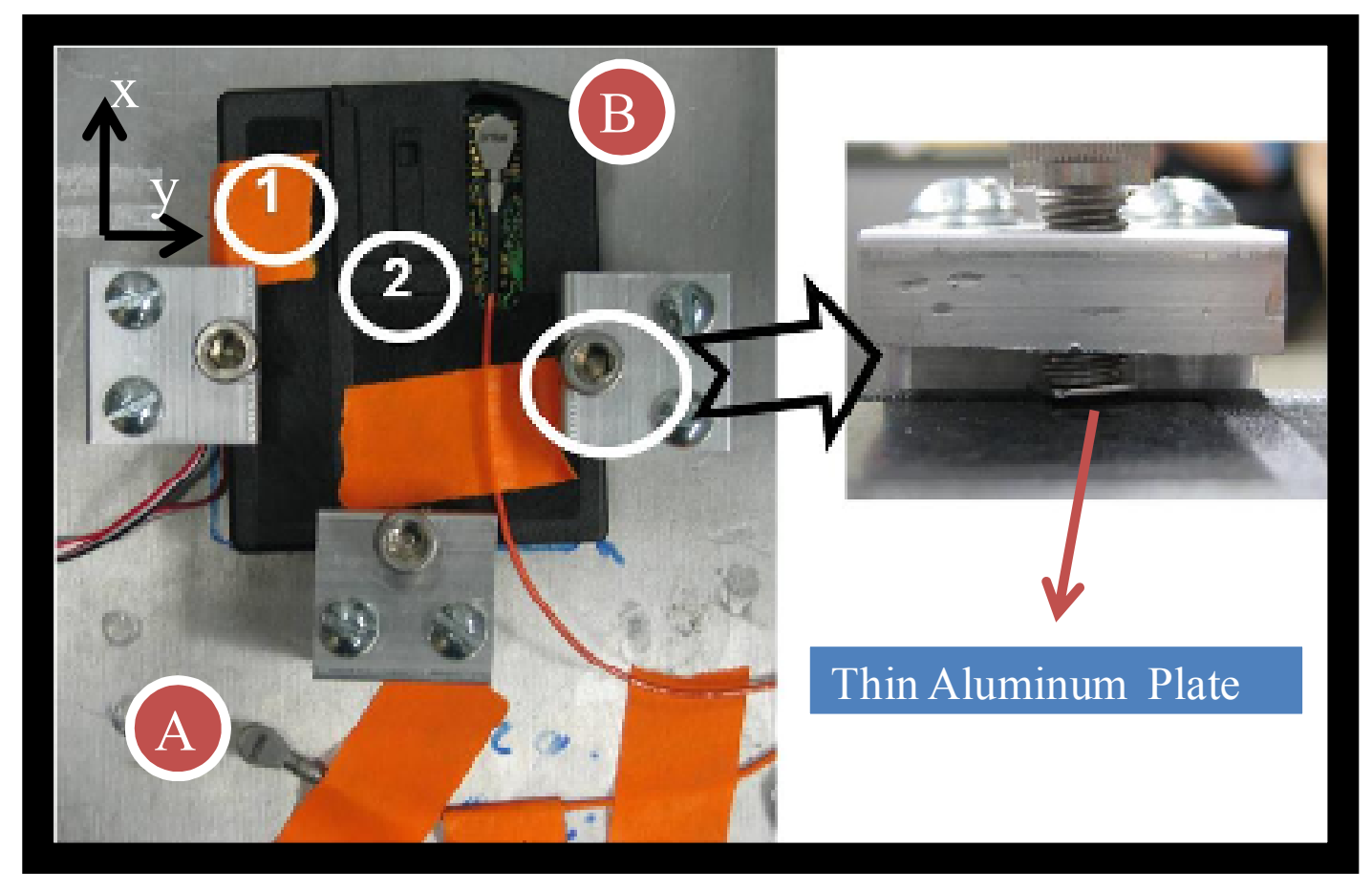

Figure 2-18 Full product shock test set up- Two strain gages are mounted on the board at Points 1 and 2 to monitor the board response. Strain is measured along the $\mathrm{x}$-direction. Accelerometer $\mathrm{A}$ and $\mathrm{B}$ monitor the response of the fixture and $\mathrm{PWB}$ respectively.

\section{$\underline{2.4 \text { Drop Test Set Up }}$}

As shown in Figure 2-19, drop tests on bare PWB and on the full product assembly are performed at three different acceleration levels. A commercial drop tester is used to conduct these tests. The drop tower is capable of generating half-sine excitation profiles. A typical operation of the drop tower involves raising the drop table to the desired height followed by the drop of the drop table along the four rigid guide rods, on to a rigid base covered by one or more layers of felt materials. The number and the thickness of the felt materials control the pulse shape and duration. Data acquisition configurations are similar to those in the earlier shock tests in all aspects except the sampling rate and filtering. Sampling rate is set at 20000 samples per second for the test and the $10 \mathrm{KHz}$ low pass filter that was used in shock tests is 
removed in drop tests. Figure 2-19 illustrates the drop tower. Drop test fixtures are discussed next in Sections 2.4.1.

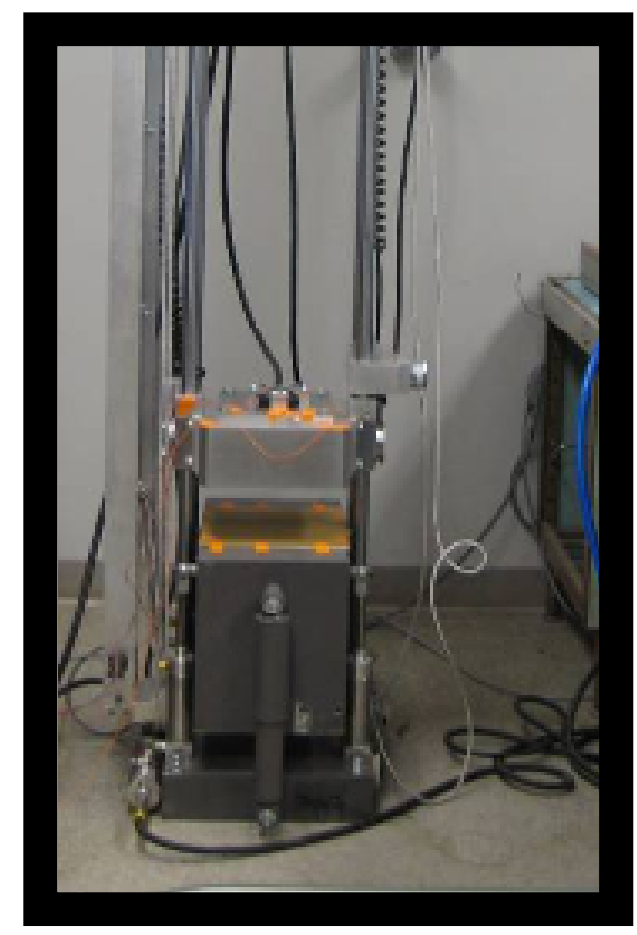

Figure 2-19-Lansmont Drop Tester

\subsubsection{Drop Test Set Up for Clamped PWB and Full Product Assembly}

The drop test fixtures for the bare PWB and for the full product assembly are identical to those used earlier in the shock tests, described earlier in Sections 2.3.1 and 2.3.5, and demonstrated in Figure 2-7 and Figure 2-18. In bare board drop test, the PWB is clamped. The main difference between the drop and shock tests is the excitation profile, which is specified in the test matrix. The bare PWB is dropped 10 times from a specified height and the results are averaged. The maximum acceleration generated as a result of drop loading is approximately 1600 Gs. The number of felt 
pads underneath the drop table is selected to maintain a pulse duration of $0.5 \mathrm{~ms}$. The instrumentation (accelerometers and strain gages) and their locations were also identical to those used earlier in the shock testing.

\section{$\underline{2.5 \text { Test Results }}$}

Section 2.5 contains the results for broad band, shock, and drop tests described in Sections 2.3 and 2.4. The shock and broad band test results are discussed first and followed by the drop test results.

\subsubsection{Broad Band and Shock Test Results}

Section 2.5.1 demonstrates the broad band and shock test results for simply and spring supported PWBs, as well as the plastic housing.

\subsubsection{Broad Band Response of Clamped and Spring-mounted PWBs}

As mentioned previously, broad band tests are performed with excitation of $0.01 \mathrm{~g}^{2} / \mathrm{Hz}$ over the frequency range of $60-800 \mathrm{~Hz}$, to find the natural frequencies of the PWBs with and without the spring-mounted boundary condition. As shown in Figure 2-20, the Fast Fourier Transform (FFT) of the response shows that the first and second natural frequencies of the clamped PWB occur at $231 \mathrm{~Hz}$ and $355 \mathrm{~Hz}$, respectively. In a similar manner the first two natural frequencies of the springmounted PWB are found to be $262 \mathrm{~Hz}$ and $390 \mathrm{~Hz}$, respectively, as shown in Figure 2-21. As expected, the addition of the spring-mounted boundary condition raises the natural frequencies by about $9-13 \%$. 


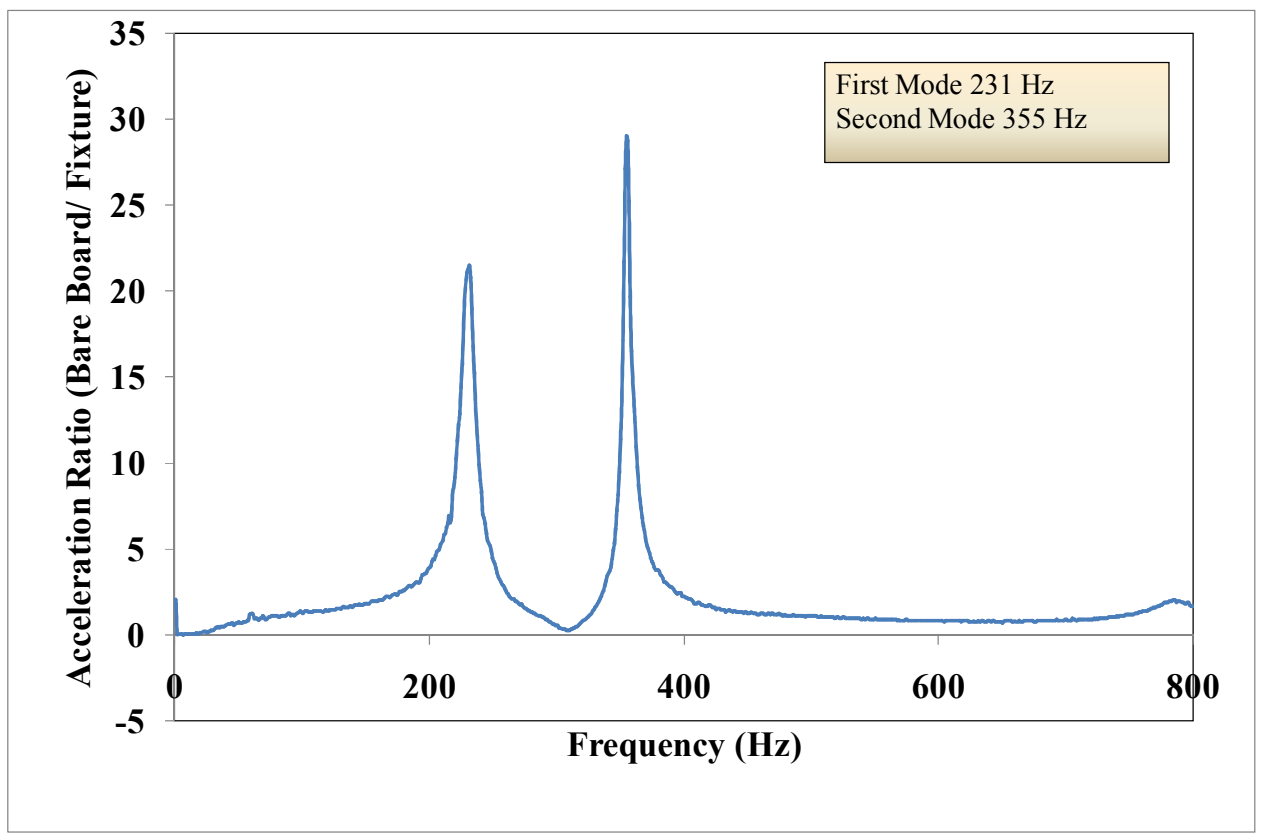

Figure 2-20 Frequency response function of clamped PWB

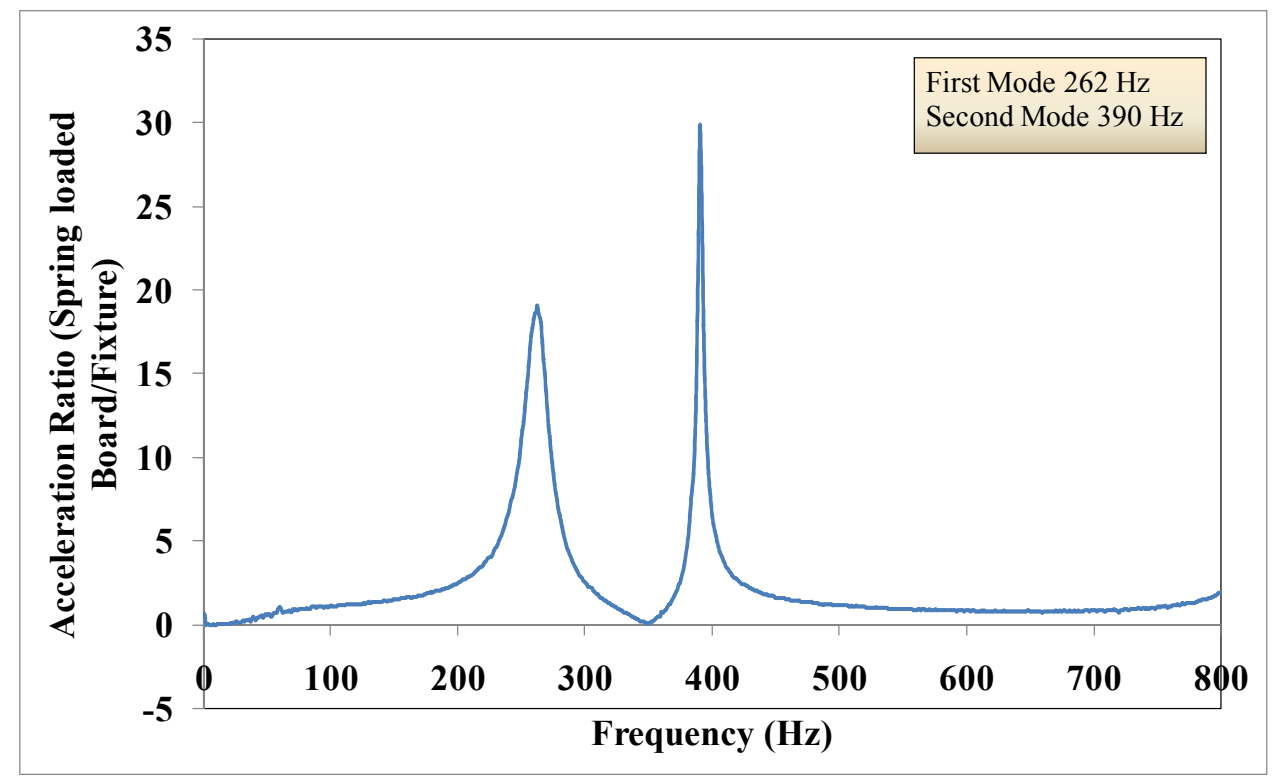

Figure 2-21 Frequency response function of spring-mounted PWB

\subsubsection{Shock Response of Clamped PWB}

The procedure and test set up for half-sine classical shock test have been explained in detail earlier, in Sections 2.3.1. Figure 2-22 compares the acceleration profiles of the PWB measured at the free end of the PWB as shown in Figure 2-7 and 
of the fixture. These results show a magnification from the peak excitation acceleration of 35 Gs (measured at the fixture) to the peak response acceleration of 45 Gs (measured at the free end of the PWB).

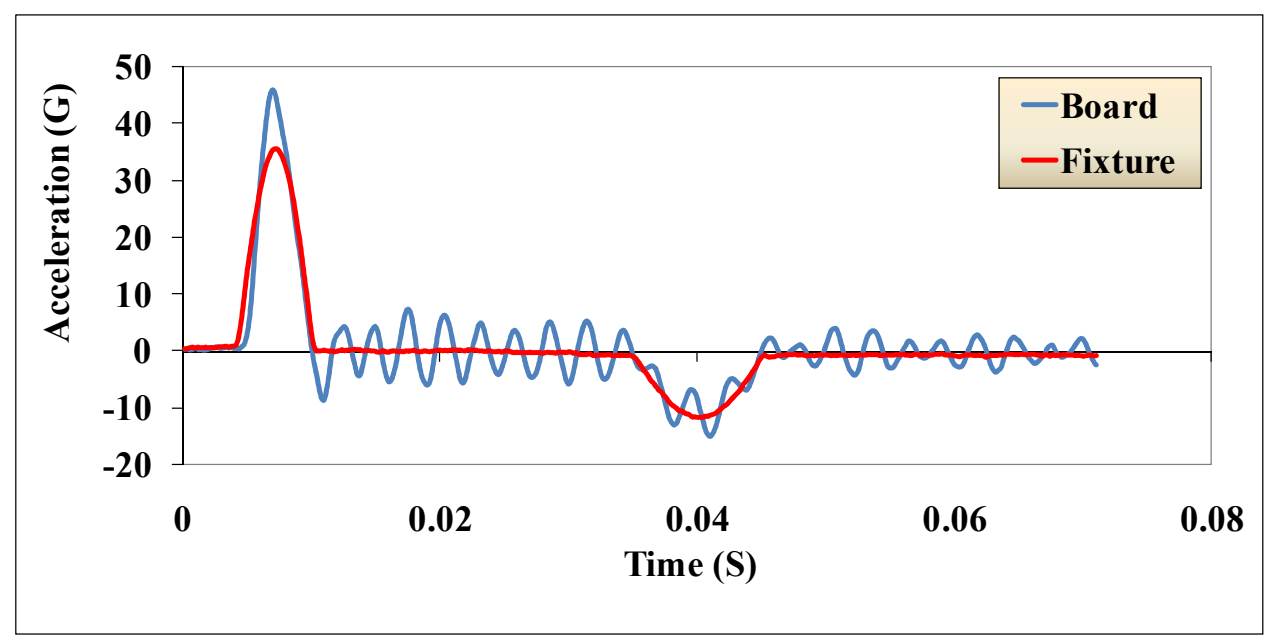

Figure 2-22 Shock response of clamped PWB

To ensure repeatability, each test was conducted several times. In Figure 2-23, each square represents the acceleration magnification factor for each test. As shown, the squares fall on top of each other which is a good indication of repeatability. Additionally, Figure 2-23 indicates that acceleration magnification factor is roughly 1.4 for $17 \mathrm{G}$ and $35 \mathrm{G}$ shock excitation. The acceleration magnification factor appears to marginally increases at $60 \mathrm{G}$ excitation, possibly due to nonlinearities. 


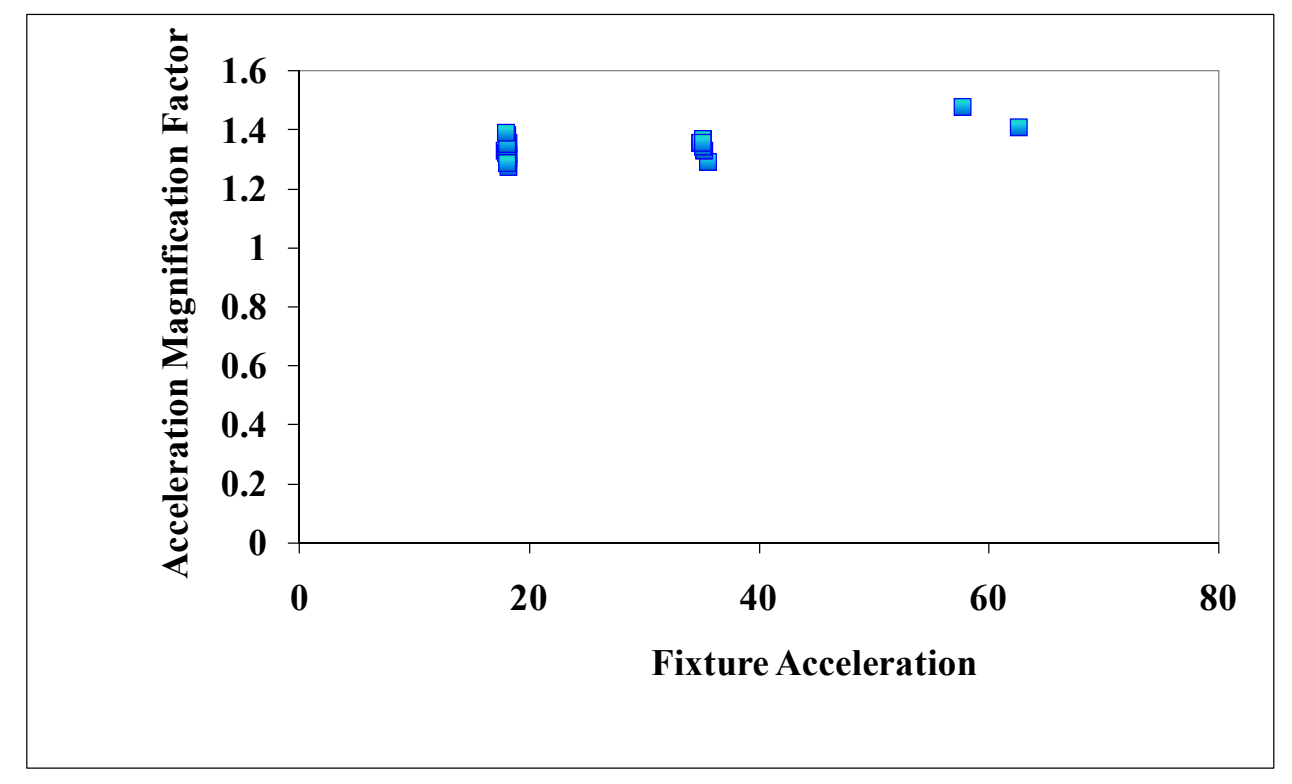

Figure 2-23- Acceleration magnification factor for clamped PWB

As explained in Sections 2.3.1 the flexural strain at the posts is notably higher than the strain levels at other locations, due to the localized constraints. Figure 2-24 demonstrates the strain history at the mounting post of the clamped PWB (The strain gage is marked with a red circle in Figure 2-24) for $35 \mathrm{G}$ shaker excitation. The peak flexural strain $\varepsilon_{\mathrm{XX}}$ at this location is 136 micro-strains.

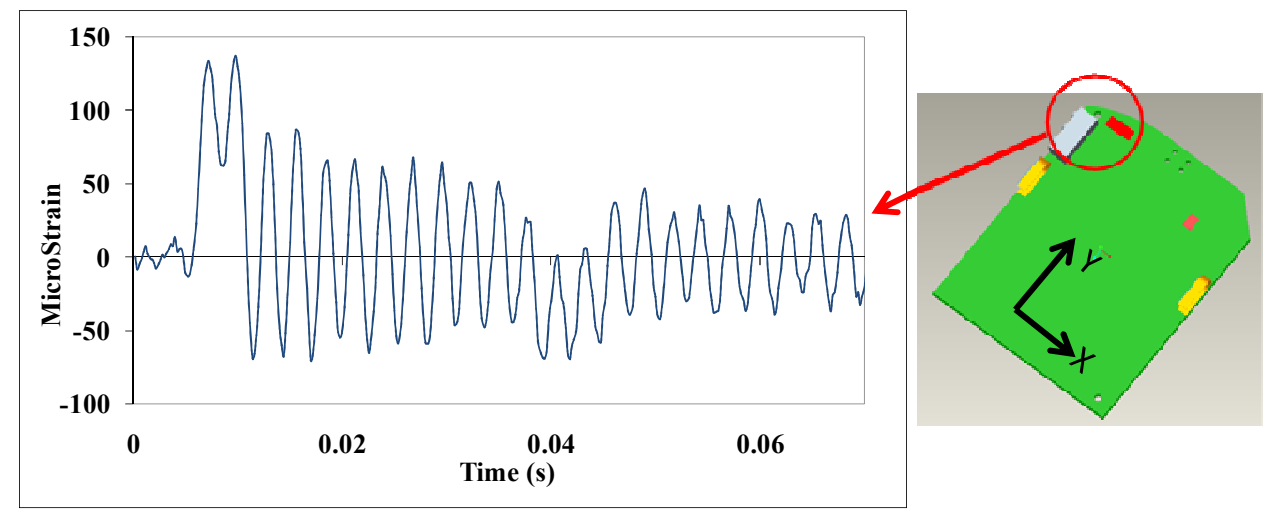

Figure 2-24 Flexural strain response $\varepsilon_{x x}$ on clamped PWB at the location shown in the Figure

Figure 2-25, illustrates the acceleration frequency response of the PWB measured at the accelerometer location shown in Figure 2-7. The first two excitation 
peaks, which occur at $254 \mathrm{~Hz}$ and $372 \mathrm{~Hz}$ respectively, are reasonably close to that of obtained from the broad band test. The slight difference is possibly due to the low resolution of the frequency response function $( \pm 20 \mathrm{~Hz})$. Additionally the FFT of the strain history is plotted in Figure 2-25. The results indicate that the second mode of the PWB (which occurs at $352 \mathrm{~Hz}$, as shown in Figure 20) is the dominant mode in this case, although freely vibrating systems are usually expected to respond predominantly at their lowest natural frequency.

This discrepancy can be explained by examining the shock spectrum of the fixture on which the board sits. The force exerted on the fixture is a half-sine force pulse that can be defined by the following function [6]:

$f(t)=F_{0} \sin \left(w_{0} t\right)\left[u(t)-u\left(t-\frac{\pi}{w_{o}}\right)\right]$

Equation 2.1

Where the pulse duration is $t_{0}=\pi / w_{0}$. The corresponding spectrum of the half sine pulse can be obtained by first taking the Laplace transform of $f(t)$ and then by setting $s=j \omega$. The end product is the amplitude density spectrum of the half-sine force pulse and it is represented by $\mathrm{G}_{\mathrm{hs}}(w)$ :

$G_{h s}(w)=\frac{2 F_{0}}{w_{0}}\left|\frac{\cos \left(\frac{\pi w}{2 w_{0}}\right)}{1-\left(\frac{w}{w_{0}}\right)^{2}}\right|$

Equation 2.2

Equation 1.2 is plotted in Figure 2-26. The graph indicates that the half-sine force pulse does not excite the system at $\left(w / w_{0}\right)=(2 k+1)$ where $k$ is a real number bigger than one. In other words, the spectral content of the excitation energy has periodic peaks and notches in the frequency domain. All modes that coincide with the peaks of the frequency response function (FRF) will be preferentially excited, 
while the modes that coincide with the notches in the excitation FRF will not be excited.

In the case of the shock test of the clamped PWB, the pulse duration is set to 6ms. Thus, $w_{0}$ can be calculated from $\mathrm{t}_{0}=\pi / w_{0}$ which is equal to $523.6 \mathrm{Rad} / \mathrm{s}$. Furthermore, Figure 26 shows that $\mathrm{G}_{\mathrm{hs}}$ vanishes, at $\left(w / w_{0}\right)=3$. Combining this with the value of $w_{0}$ above, we obtain a value of $w=250 \mathrm{~Hz}$ for the first notch in the frequency spectrum for our shock excitation. This frequency is very close to the first natural frequency of the PWB, hence the first modal response of the PWB is negligible in comparison to the second mode.

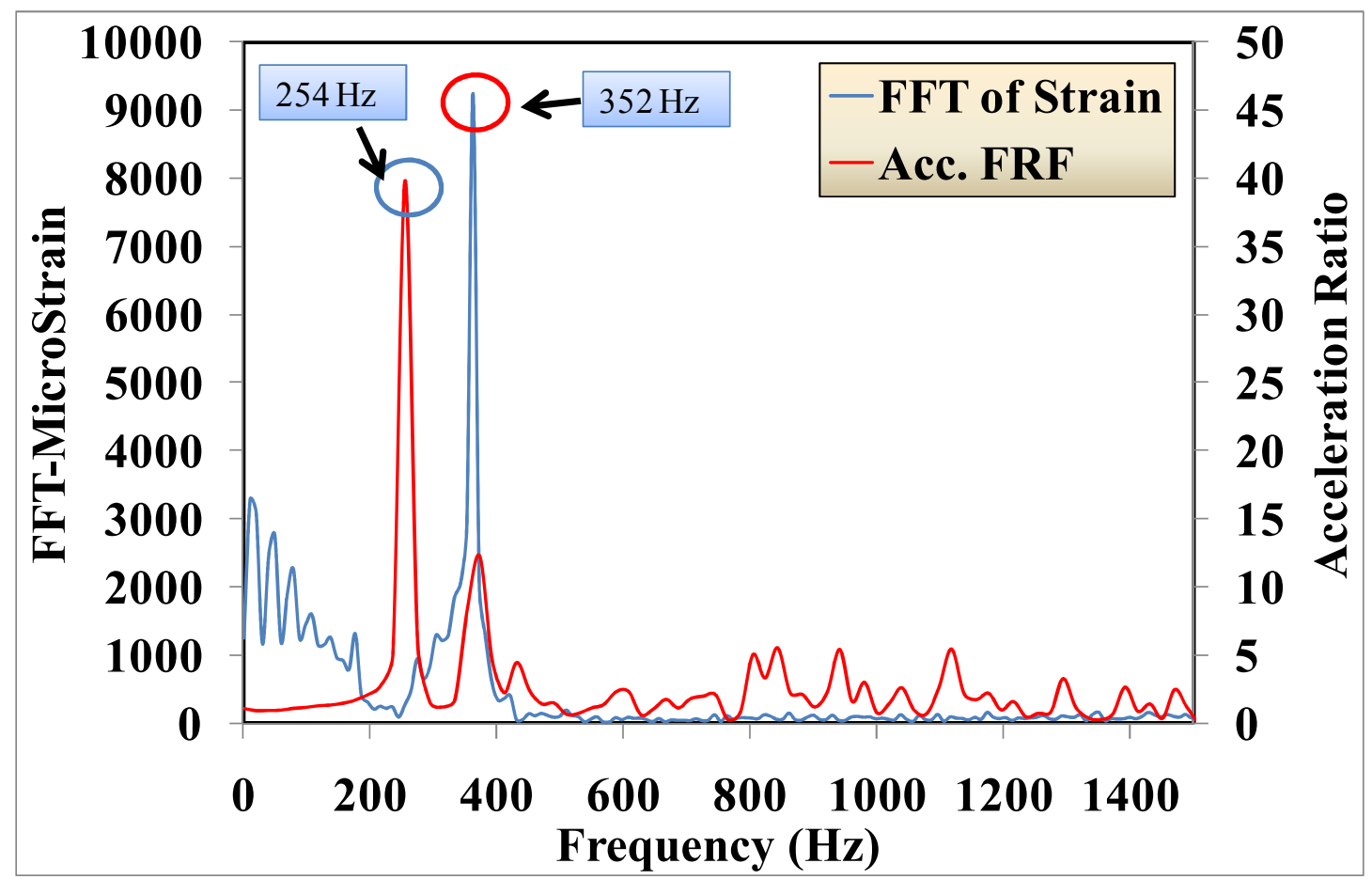

Figure 2-25 FFT response of the strain Gage, which is marked with a red Circle in Figure 2-24, to the half sine shock loading on clamped PWB. Frequency response function of the accelerometer on the free end of the PWB as shown in Figure 2-7. 


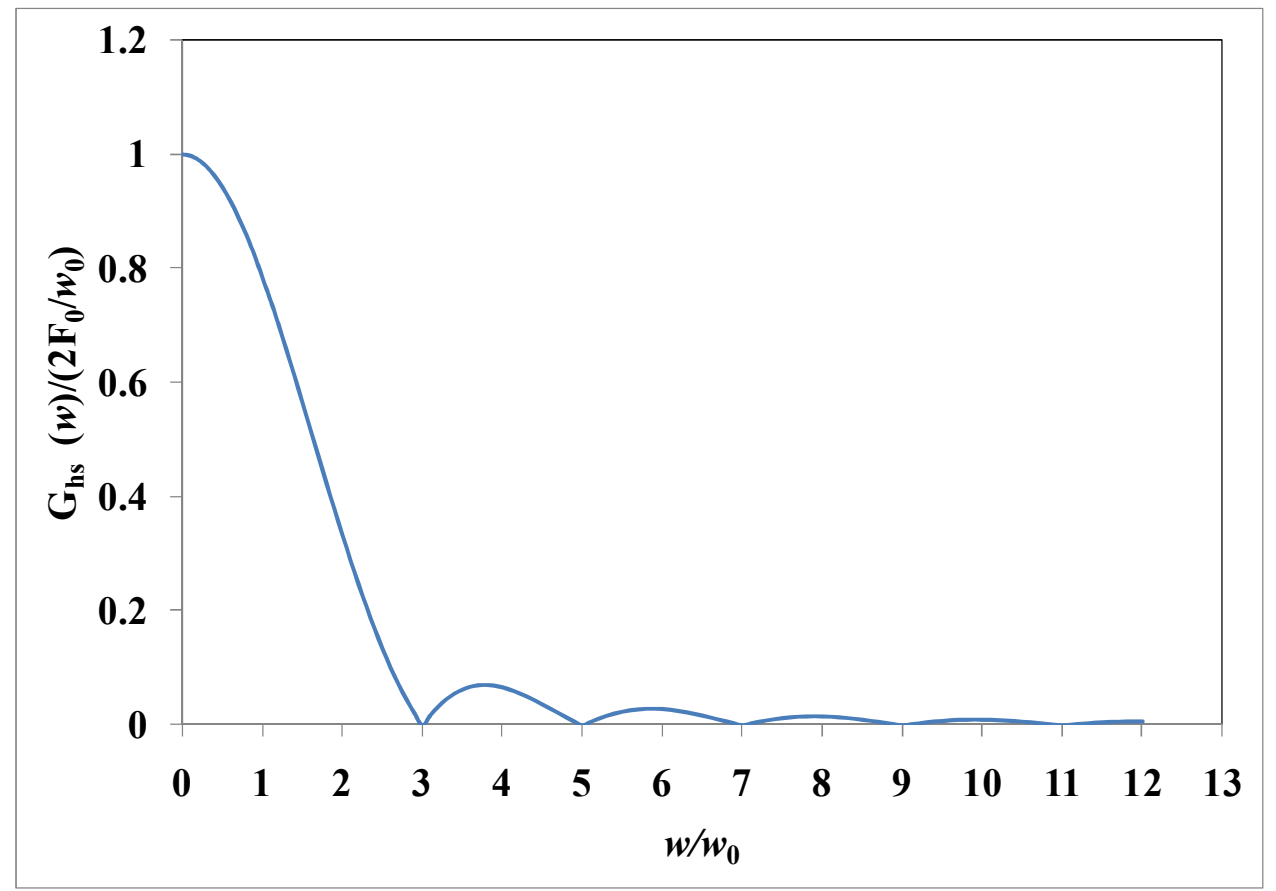

Figure 2-26- Amplitude Density Spectrum of Half-Sine Wave Pulse of Duration $\pi / w_{0}$

Alternatively, the theoretical results obtained above can be confirmed experimentally by plotting the FFT response of the fixture to the half-sine force pulse. This graph is shown in Figure 2-27. As demonstrated in Figure 2-27, the base fixture does indeed fail to provide excitation energy to the PWB at its first natural frequency. The additional notches in the shock spectrum of the input pulse marked with a red rectangle are due to the shock 'relaxation' typically observed in shock testing with electro dynamic shakers. These low frequency perturbations of the shock spectrum can filter out the low frequency response of the system. 


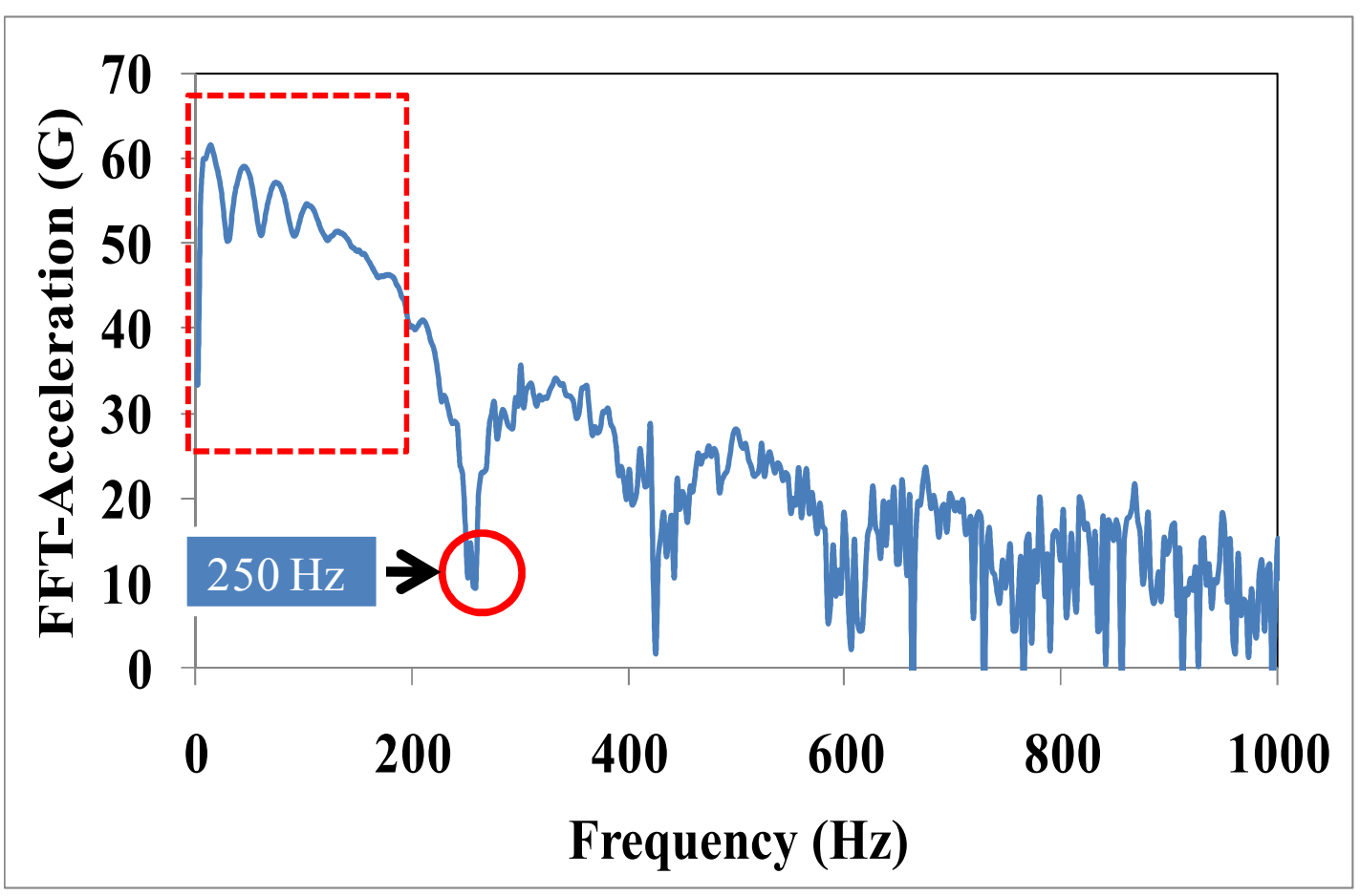

Figure 2-27 FFT of the fixture motion in response to the half sine shock loading

\subsubsection{Empty Case Broad Band Test Results}

Before performing broad band test on the empty housing, it is important to make sure the fixture natural frequencies do not lie within the frequency range of the excitation energy in the test. Figure 2-28, illustrates the FFT response of the accelerometer placed on the fixture, shown in Figure 2-12, to broad band excitation. It is apparent from the graph that the first three natural frequencies of the fixture occur in the region between $2000-3000 \mathrm{~Hz}$. Therefore, all test data beyond $2000 \mathrm{~Hz}$ is ignored throughout this study. 


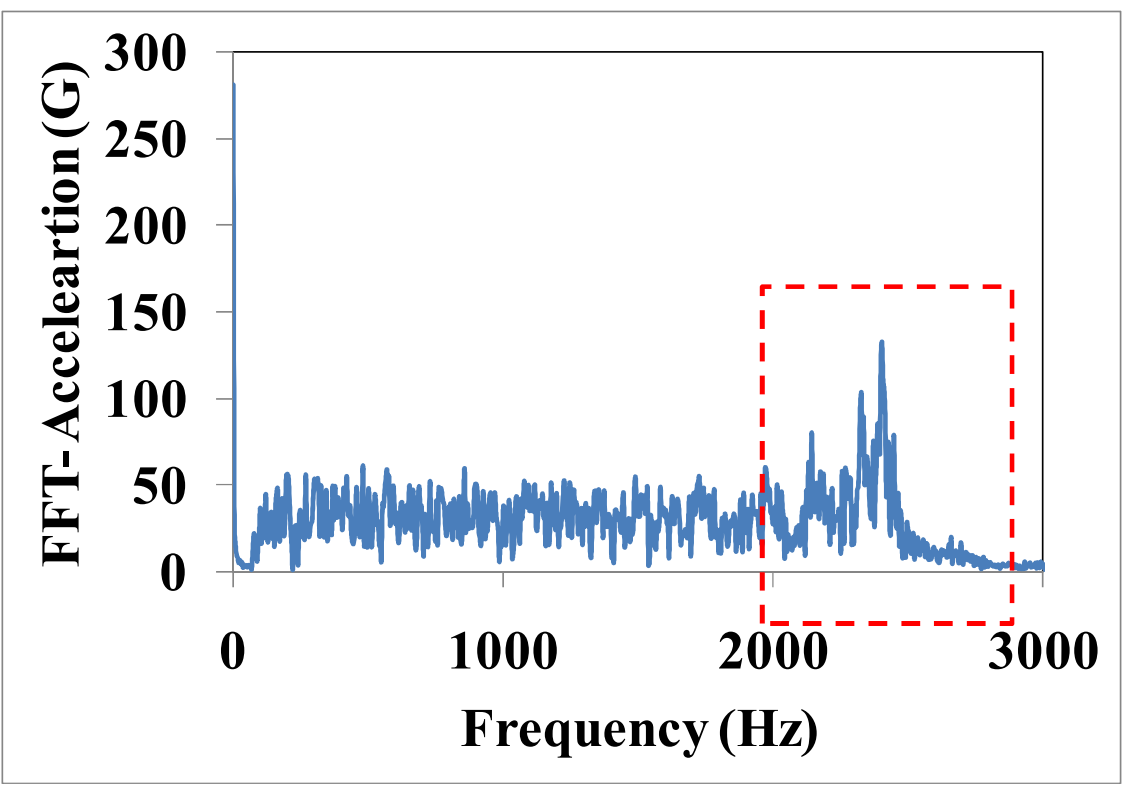

Figure 2-28 Frequency response function of the fixture used for broad-band test of the empty housing. The accelerometer is placed on the base of the fixture as shown in Figure 2-12. Due to the fixture excitation peaks that occur after $2000 \mathrm{~Hz}$, all test data will be ignored in the region marked with a red dotted rectangle.

Figure 2-29 shows the frequency response function of the empty case with respect to the fixture at the two accelerometer locations shown in the figure. First and second natural frequencies of the empty case take place at $723 \mathrm{~Hz}$ and $1016 \mathrm{~Hz}$, respectively. Ultimately, the goal in this study is to capture the same natural frequencies for the empty case with finite element modeling. In order to accomplish this task, it is very essential to ensure that the same mode shapes are obtained later in the finite element modeling conducted in Part II of this study. As discussed in Section 2.3.3, it is possible for the empty case to exhibit different mode shapes, if the double sided tapes do not completely fix the bottom of the empty case to the fixture. The possible mode shapes for the empty case are illustrated in Figure 2-13 and Figure 2-14. It can be concluded from these figures that the first mode remains the same regardless of the extent to which the bottom housing is constrained. Therefore, two 
accelerometers (designated ' $\mathrm{T}$ ' and 'B' accelerometers) are used, on the top housing, as shown in Figure 2-29, to verify the mode shapes. According to Figure 2-13 and Figure 2-14, both accelerometers should always be in phase at the first mode. However, for the second mode, these two accelerometers could be either in phase or out of phase depending on the boundary conditions at the bottom of the housing. In accordance with Figure 2-13, the accelerometers are out of phase in the second mode if the entire bottom housing is fixed. On the contrary, the accelerometers remain in phase if the bottom housing is partially fixed. In the partially fixed case, the bottom housing is only fixed along its edges.

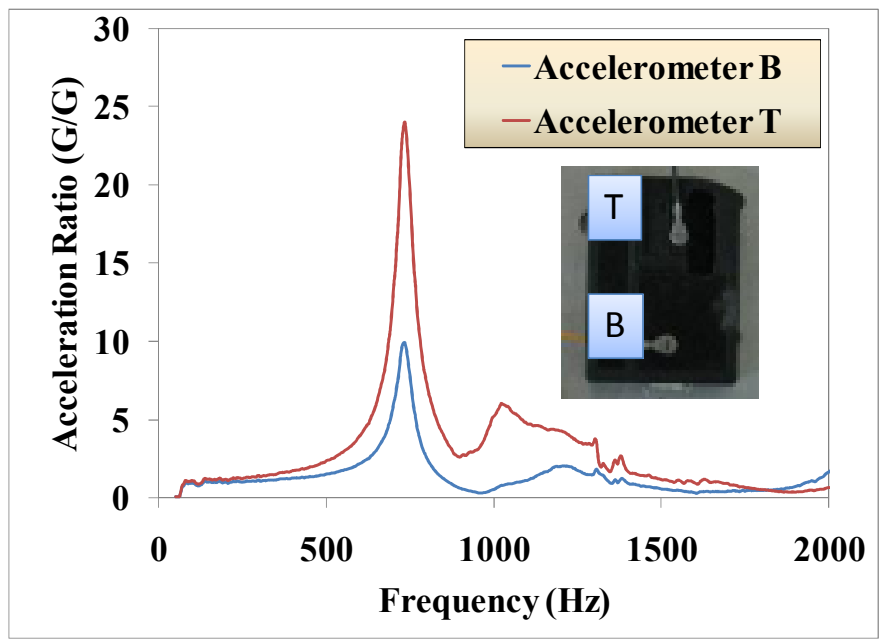

Figure 2-29 Frequency response function of the empty case at the two accelerometer locations in the figure

As marked in Figure 2-30, the phase difference between the $\mathrm{T}$ and $\mathrm{B}$ accelerometers is $360^{\circ}$ for the first mode, which specifies that both accelerometers are in phase, as expected. The phase difference, however, is $150^{\circ}$ for the second mode. The accelerometers can be considered to be almost out of phase for this mode, which corresponds to a fully-fixed boundary condition on the bottom of the housing. It is 
believed that the slight deviation from $180^{\circ}$ phase difference may be due to shaker movements.

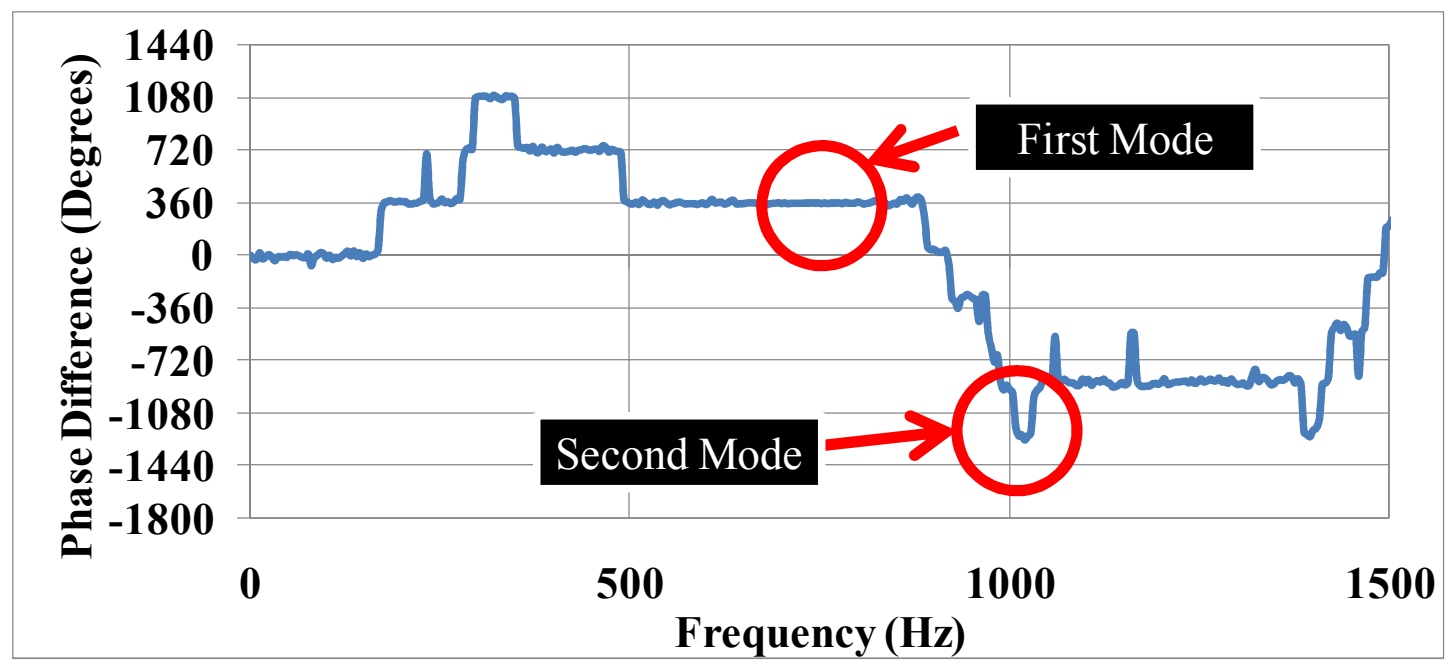

Figure 2-30 Phase Plot between Top and Bottom Accelerometers

The next Section discusses the response of the full product assembly to broad band excitation. To fully understand the contribution of individual components of the full product to the natural modes of the full system, it is important to investigate how components of the system behave individually when subjected to similar boundary conditions experienced in the full product assembly. In this section, the bottom of the plastic housing was fixed to the fixture and subsequently, the natural frequencies of the empty housing were obtained. However, since the full product is fixed to the fixture with three clamping fingers, it is imperative to investigate how the clamping fingers affect the natural frequencies of the empty housing. Figure 2-31 illustrates the natural frequencies of the clamped empty housing versus those obtained in this section. As expected, the clamping fingers stiffen the empty housing, hence higher natural frequencies. The first natural mode is increased from $723 \mathrm{~Hz}$ to $836 \mathrm{~Hz}$. The second natural mode is increased from $1016 \mathrm{~Hz}$ to $1024 \mathrm{~Hz}$. It seems that the 
clamping mechanism affects the first mode more than it does the second mode. This is possibly due to the relationship between the mode shapes and the location of the clamping fingers.

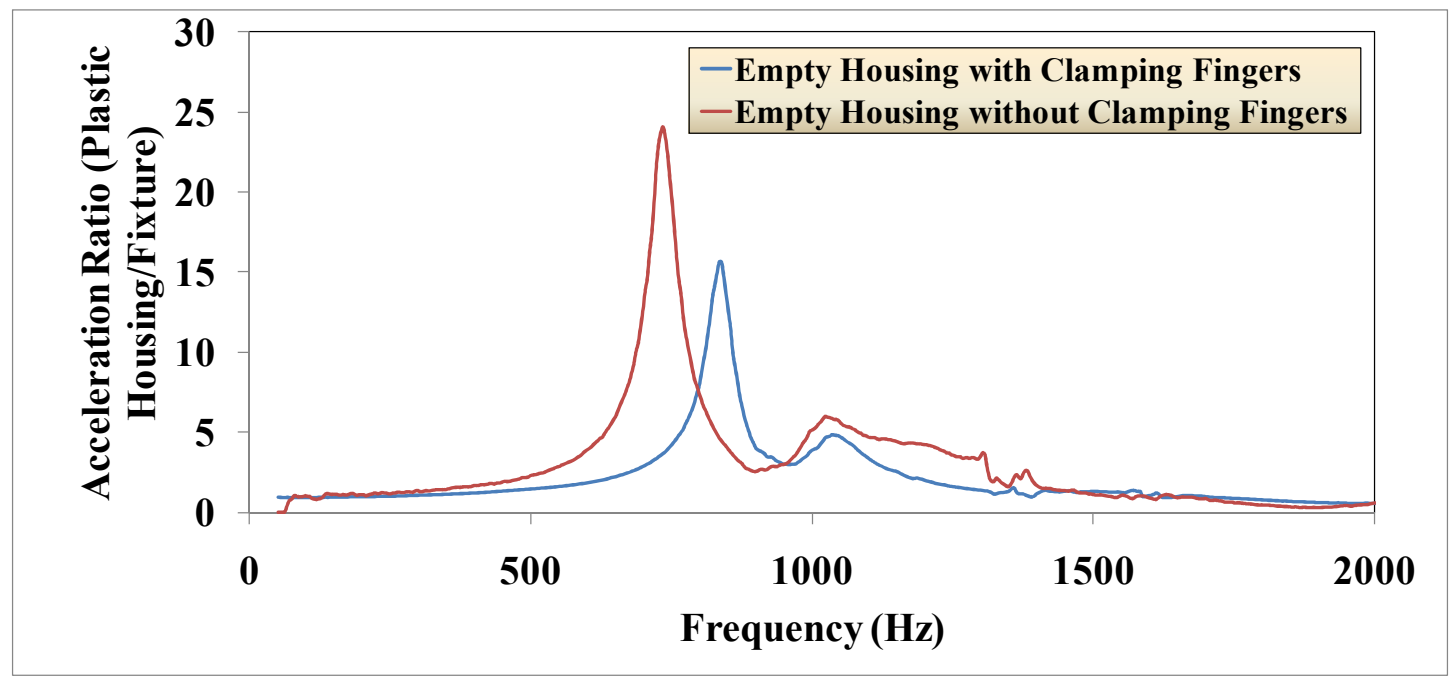

Figure 2-31 Frequency response function of the empty housing with clamping fingers versus the empty housing without the fingers. The accelerometer location for the case with the clamping fingers is shown in Figure 2-16. The accelerometer location for the case without the clamping fingers is at point $T$ as shown in Figure 2-29.

\subsubsection{Broad Band Test Results for Full Product Assembly}

As mentioned in Section 2.3.4, it is necessary to find the natural frequencies of the clamping fingers before analyzing the frequency response function of the full product assembly. Figure 2-32 illustrates the frequency response function of the accelerometer placed on top of the clamping finger as shown in Figure 2-15. It is evident that the first excitation peak occurs at $2300 \mathrm{~Hz}$. It was established in the previous section, Section 2.5.1.3, that all test results beyond $2000 \mathrm{~Hz}$ must be ignored. Therefore, the natural frequencies of the clamping fingers do not lie in the region of interest between $0-2000 \mathrm{~Hz}$.

The first four natural modes of the system are shown in the figure. In the previous section, the natural frequencies of the clamped empty housing were found to 
be $836 \mathrm{~Hz}$ and $1024 \mathrm{~Hz}$. Therefore, it is likely that the first two modes of the full product assembly are driven by the plastic housing and not the PWB. The frequencies of the first two natural modes are slightly different compared to those of the plastic housing. It should be noted that once the PWB is added to the empty housing both stiffness and mass of the system will change. Additionally, the interactions between the internal components of the full product are highly non-linear and affect the natural frequencies of the system. No conclusion can be drawn, at this point, on the third and forth natural modes. In part II of the study, finite element modeling will be conducted on the PWB which is subjected to similar boundary conditions to those in the full product assembly to determine whether or not the third and forth peaks were caused by the PWB.

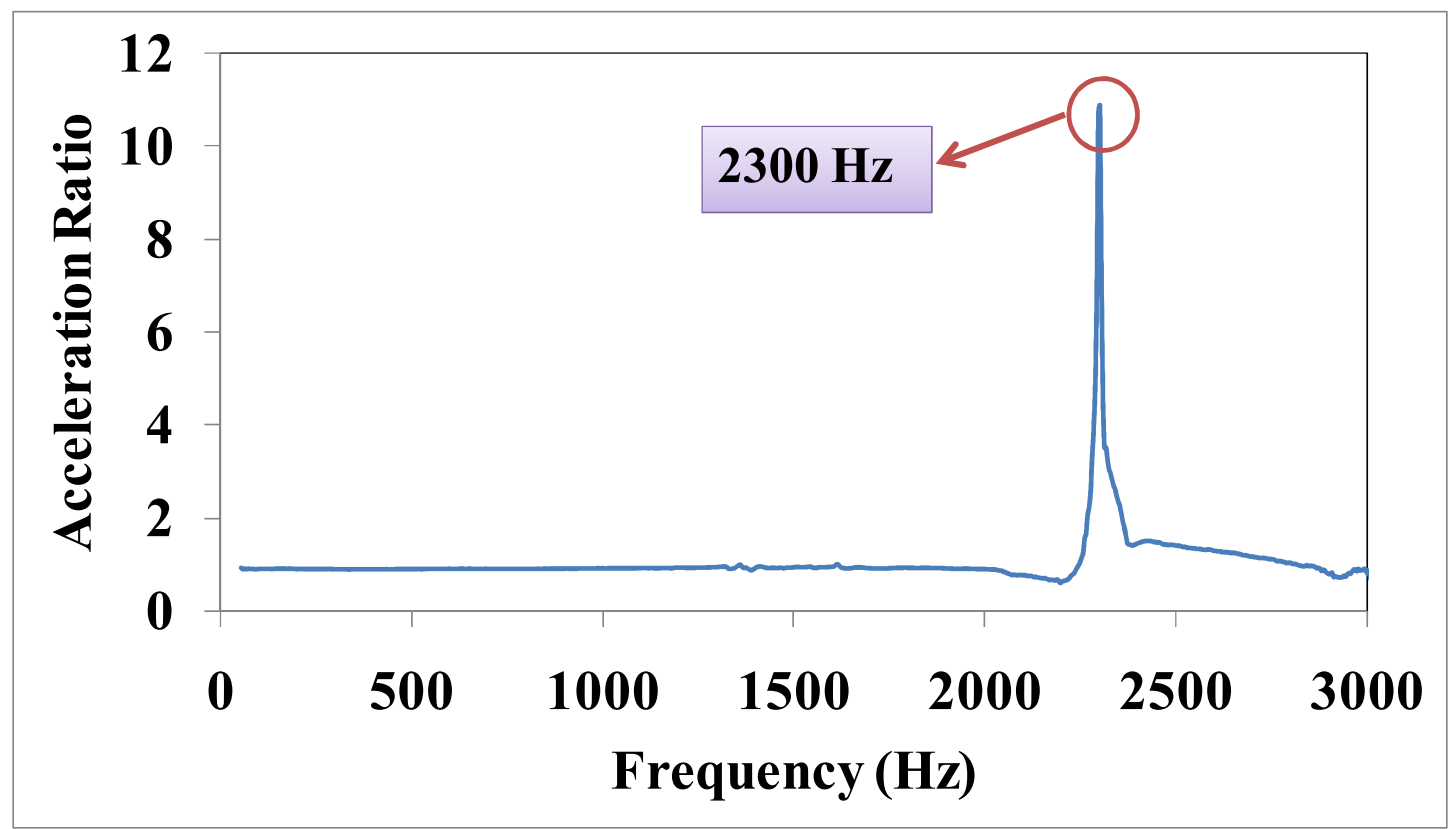

Figure 2-32 Frequency response function of the clamping fingers at the accelerometer location shown in Figure 2-15 


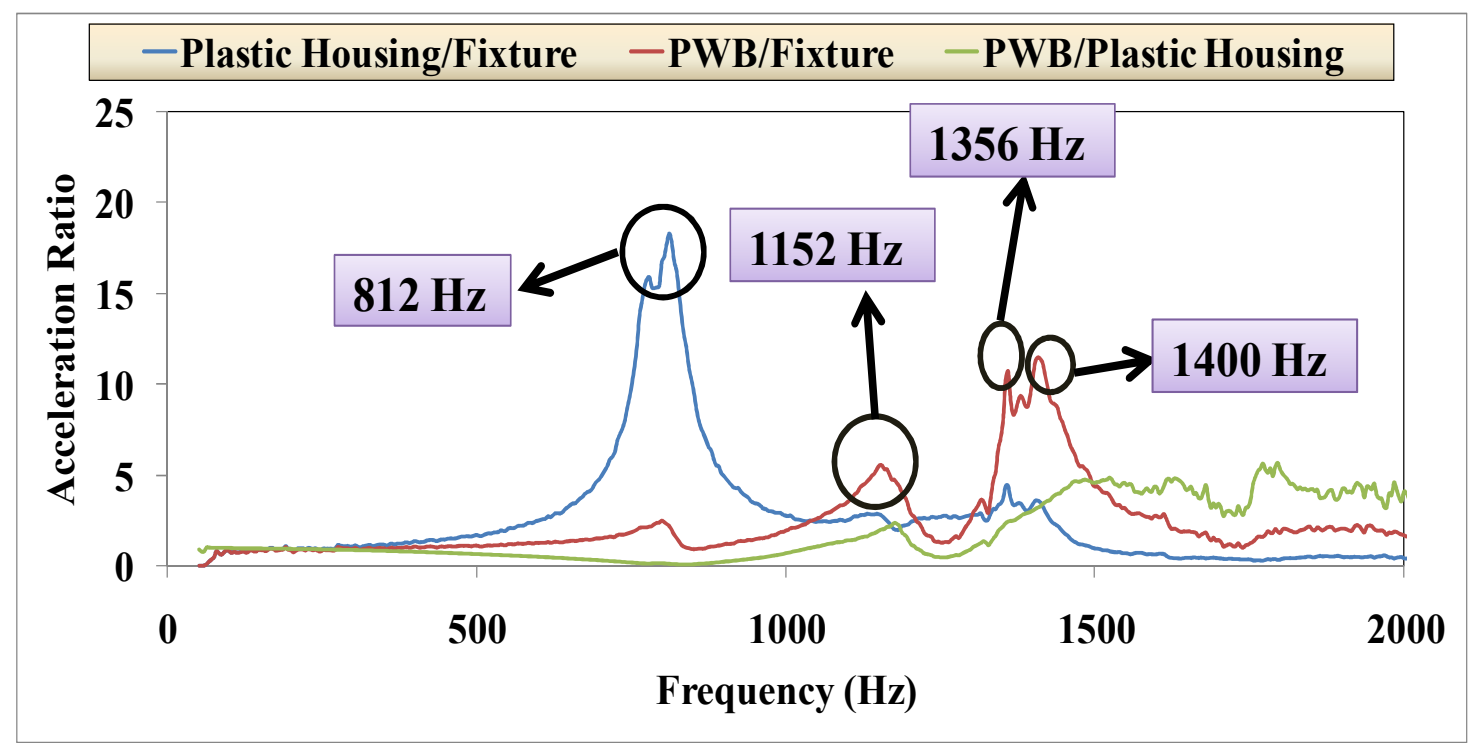

Figure 2-33 Frequency response function of the full product at the following accelerometer locations: the blue curve represents the accelerometer on the plastic housing. The red curve represents the accelerometer on the PWB. For exact accelerometers' locations on the PWB and plastic housing refer to Figure 2-17.

\subsubsection{Results of Shock Test on Full Product Assembly}

The fixture acceleration for product shock test is 30 Gs. Acceleration magnification factor measured by the accelerometer mounted on the PWB, as shown in Figure 2-18, is roughly 1.15 , which is lower than that of the clamped PWB under similar shock loading. The low acceleration magnification factor indicates that the PWB does not impact against the interior of the housing case during the test. The reduced magnification factor (compared to the clamped PWB) could be largely due to the attenuation and absorption of shock energy as it transmits through the outer plastic housing to the PWB within. Additionally, the PWB is supported differently in the housing than it is in the clamped PWB shock test. Figure 2-34 compares the fixture acceleration to that of the PWB measured at the location shown in Figure $2-18$ 


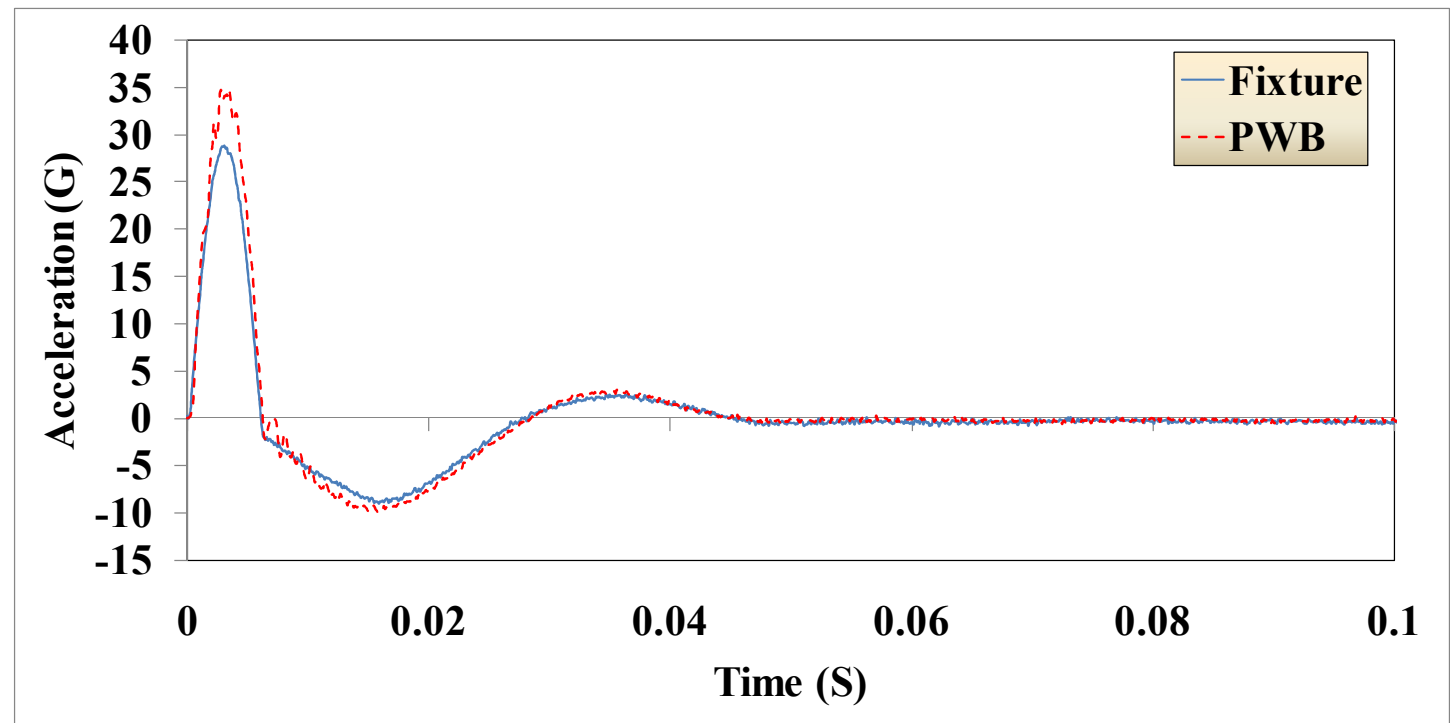

Figure 2-34 Fixture acceleration VS PWB acceleration response measured at location shown in Figure 2-18

To illustrate repeatability, the acceleration response of the PWB in the product is plotted for multiple trials in Figure 2-35. Accelerometer location is illustrated in Figure 2-18. As shown in Figure 2-35, the peak accelerations for all the trials are approximately the same and they all follow the same trend. Furthermore, strain at both measured locations (shown earlier in Figure 2-18 in Section 2.3.5) was insignificant.

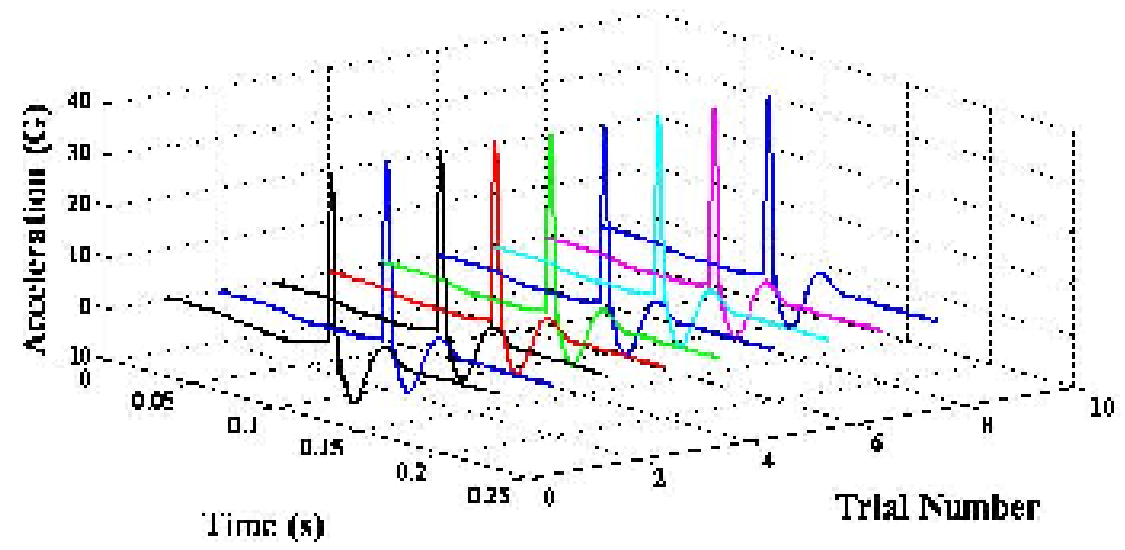

Figure 2-35 Product shock test repeatability

Figure 2-36 illustrates the FFT of the fixture motion in response to the half sine shock loading, as well as the FFT response of the accelerometer mounted on 
the PWB. Accelerometer locations are shown in Figure 2-18. As discussed in Section 2.5.1.2, the spectral content of the excitation energy has periodic peaks and notches in the frequency domain. Figure 2-36 also indicates that approximately $90 \%$ of the energy of the input pulse is pumped to the full product at low frequencies in the hatched region of Figure 2-36. Consequently, the energy transmitted to the system after the first notch is insignificant. As discussed in Section 2.5.1.5, the first natural frequency of the full product occurs far away from the hatched region. Therefore, the natural frequencies of the system are not excited due to low levels of excitation. Additionally, the existence of multiple notches in the spectral content of the input pulse contributes greatly to the disappearance of the natural frequencies of the full product. Therefore, Figure 2-36 does not provide any useful information about the locations of the natural modes of the full product assembly.

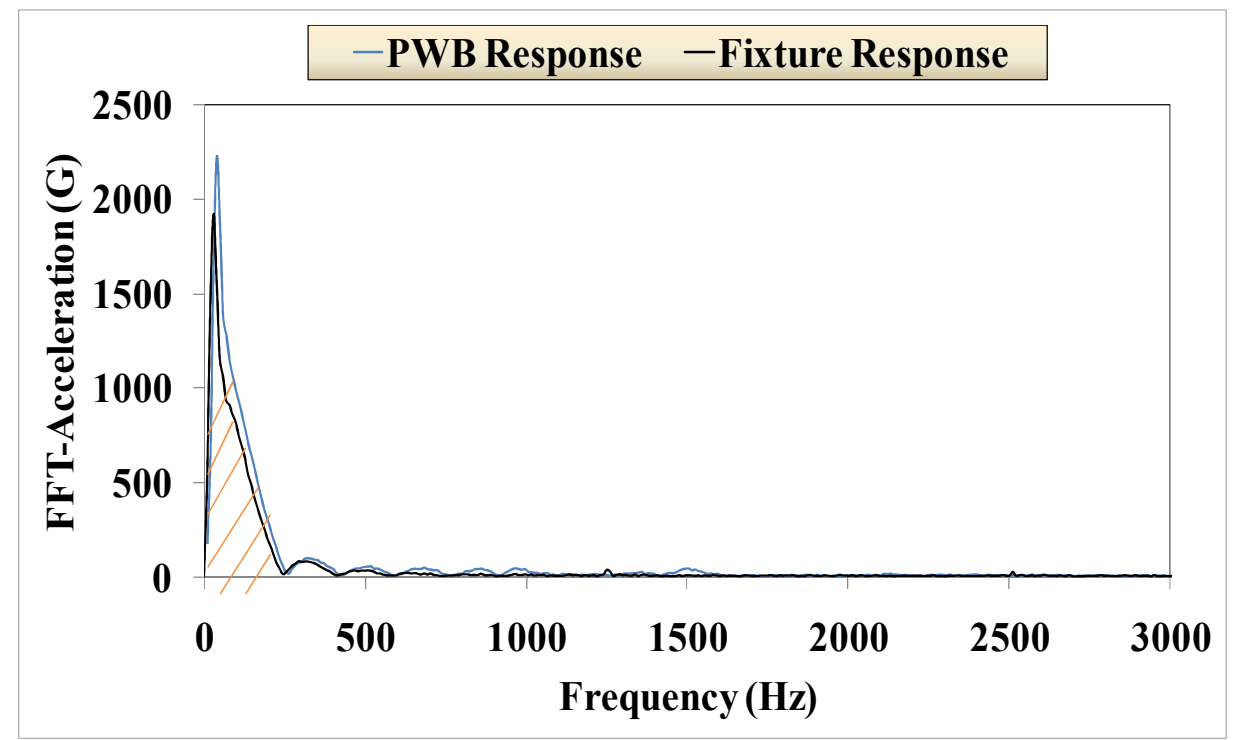

Figure 2-36 FFT of the fixture motion in response to the half sine shock loading. The notches of the frequency response function occur at $250,410580,740$, and $910 \mathrm{~Hz}$ respectively. 


\subsubsection{Drop Test Results}

Section 2.5.2 discusses drop test results for clamped PWB, as well as the full product assembly. Additionally, this section compares the drop test results with that of the shock test for both clamped PWB and full product assembly.

\subsubsection{Drop Test Results for Clamped PWB}

The fixture acceleration and the response of the clamped PWB, for one of the ten drops, are shown in Figure 2-37. The locations for the accelerometers were shown earlier in Figure 2-7of Section 2.3.1.

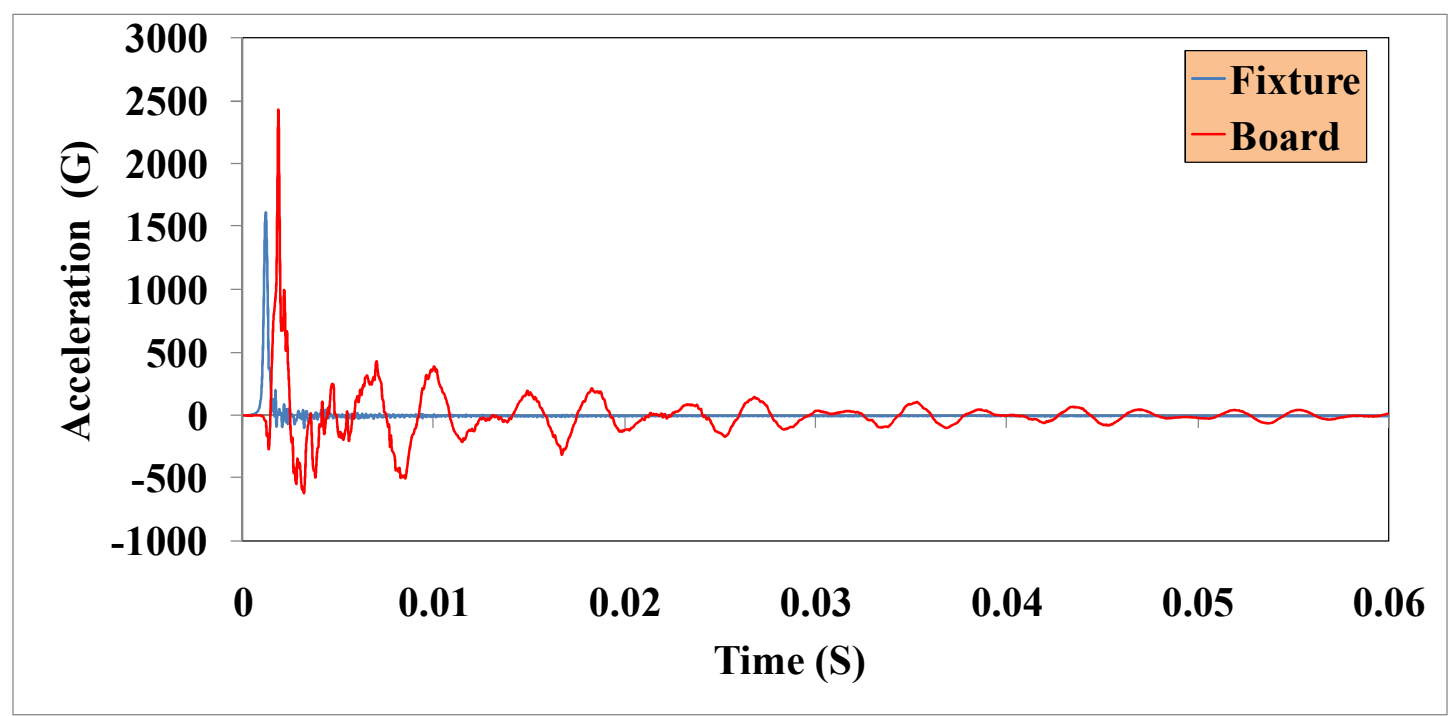

Figure 2-37 Fixture acceleration and PWB acceleration for drop test on clamped PWB

For the case shown above, the acceleration magnification is around 1.5. Figure 2-38 demonstrates that drop test is less repeatable in comparison to the shock test. The results for shock test were shown earlier in Section 2.5.1.2. Even though the fixture acceleration fluctuates by $1.5 \%$, the PWB acceleration magnification factor varies from 1.2 to 1.5 for a fixed height on the drop tower. 


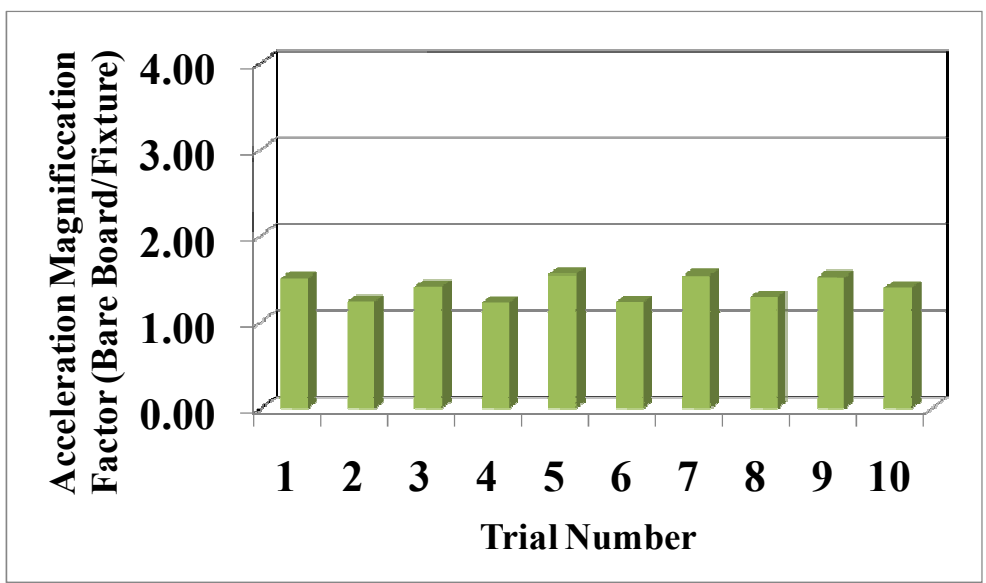

Figure 2-38 Acceleration magnification factor for drop test on clamped PWB

This variation in PWB response needs to be taken into consideration when comparing simulation results with experimental data later, in Part II of this study. Thus, the distribution that represents the peak values of each trial must be found and the accuracy of simulation results will be compared against the parameters of the experimental data distribution. If the data provided in Figure 2-39 are described with a normal distribution, then the mean and standard deviation values are $2252 \mathrm{G}$ and $217 \mathrm{G}$, respectively. 


\begin{tabular}{|c|c|c|c|}
\hline Trial No. & Fixture Acceleration (G) & Board Acceleration (G) & Acceleration Ratio \\
\hline 1.00 & 1612 & 2427 & 1.51 \\
\hline 2.00 & 1620 & 2004 & 1.24 \\
\hline 3.00 & 1597 & 2248 & 1.41 \\
\hline 4.00 & 1644 & 2006 & 1.22 \\
\hline 5.00 & 1639 & 2549 & 1.56 \\
\hline 6.00 & 1619 & 1987 & 1.23 \\
\hline 7.00 & 1611 & 2481 & 1.54 \\
\hline 8.00 & 1633 & 2108 & 1.29 \\
\hline 9.00 & 1616 & 2455 & 1.52 \\
\hline 10.00 & 1621 & 2261 & 1.40 \\
\hline
\end{tabular}

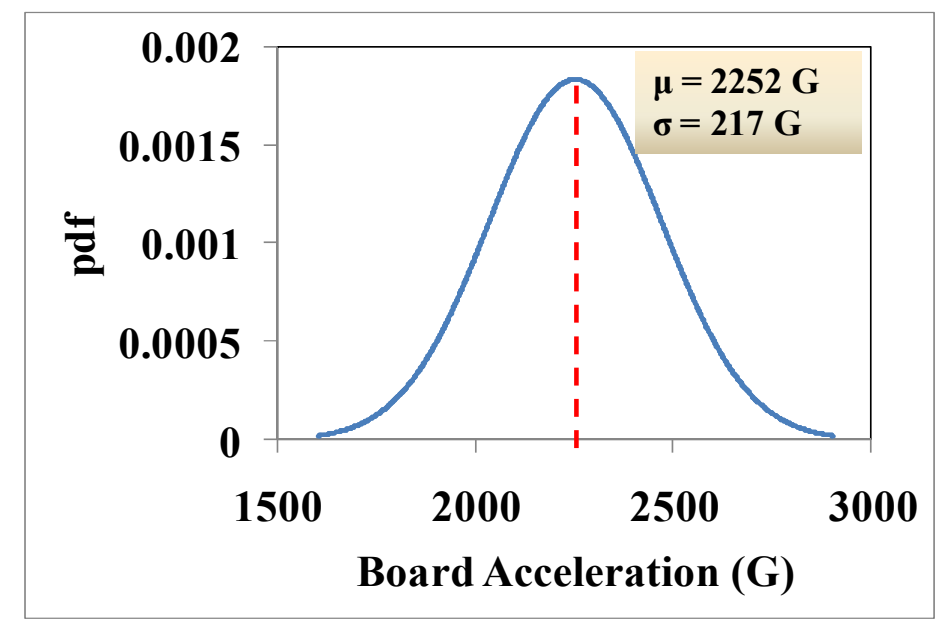

Figure 2-39- Bare Board acceleration response can be described with a normal distribution with mean and standard deviation values of $2252 \mathrm{G}$ and $217 \mathrm{G}$, respectively..

Strain response of the clamped PWB at the post (designated as Point A in Figure 2-40) is shown in Figure 2-40. The peak strain at this point is approximately 4200 microstrains for a $1600 \mathrm{G}$ drop. 


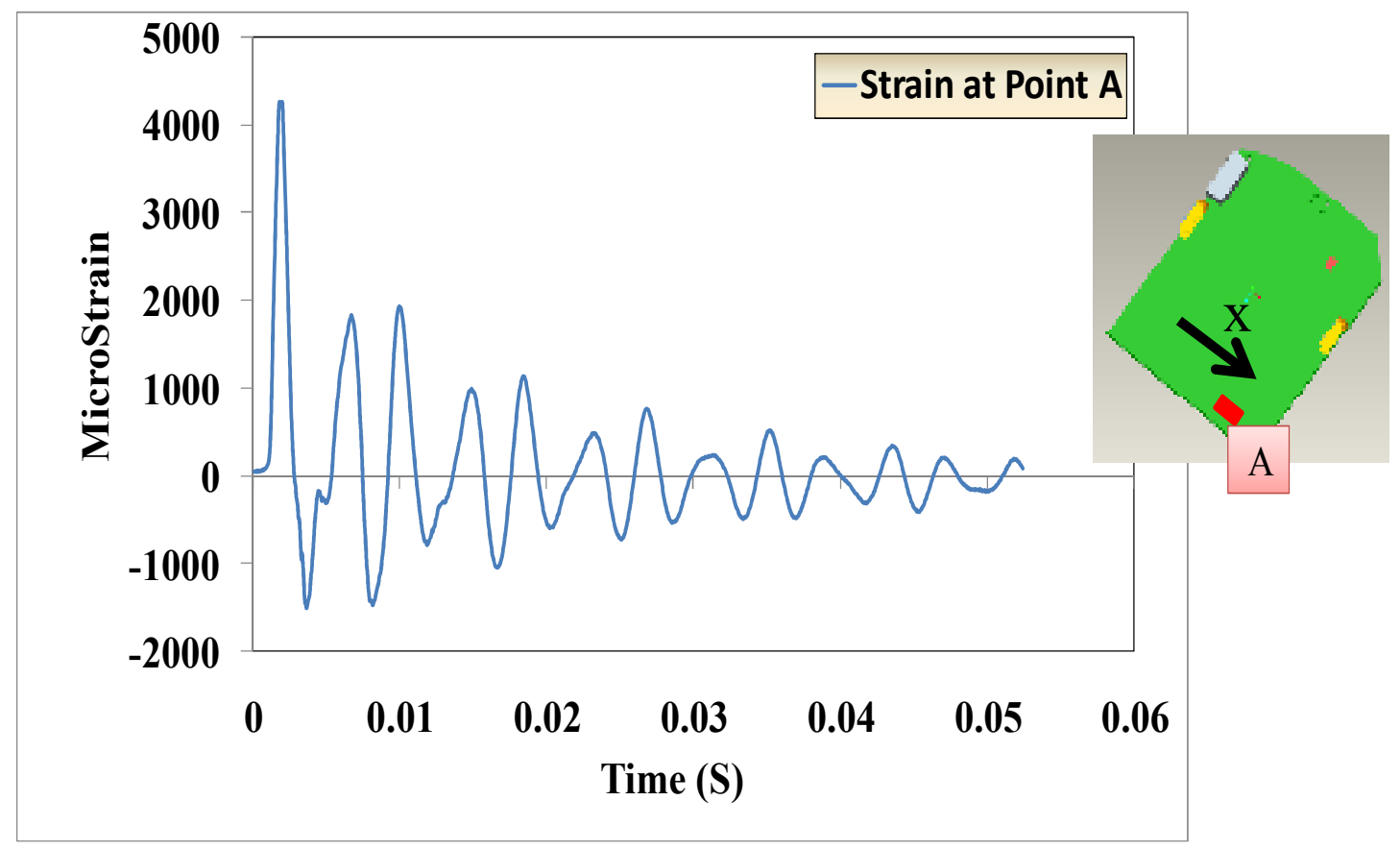

Figure 2-40- Strain response at point A measured in the x-direction, during drop test of clamped PWB

The shock spectrum of the drop table half-sine force pulse is plotted in Figure 2-41. Unlike, the shock test results reported earlier in Section 2.5.1.2, both natural modes of the board are excited in drop loading, because the significantly shorter pulse duration in the drop test does not produce any notches in the excitation spectrum at the first natural mode of the clamped PWB. Figure 2-42 and Figure 2-43 demonstrate the FFT response of the strain gage, shown in Figure 2-40, and the frequency response function of the accelerometer placed on the free end of the PWB to the drop half sine pulse. The frequencies at which the first two modes occur are in agreement with shock and broad-band test results presented in Section 2.5.1.2. 


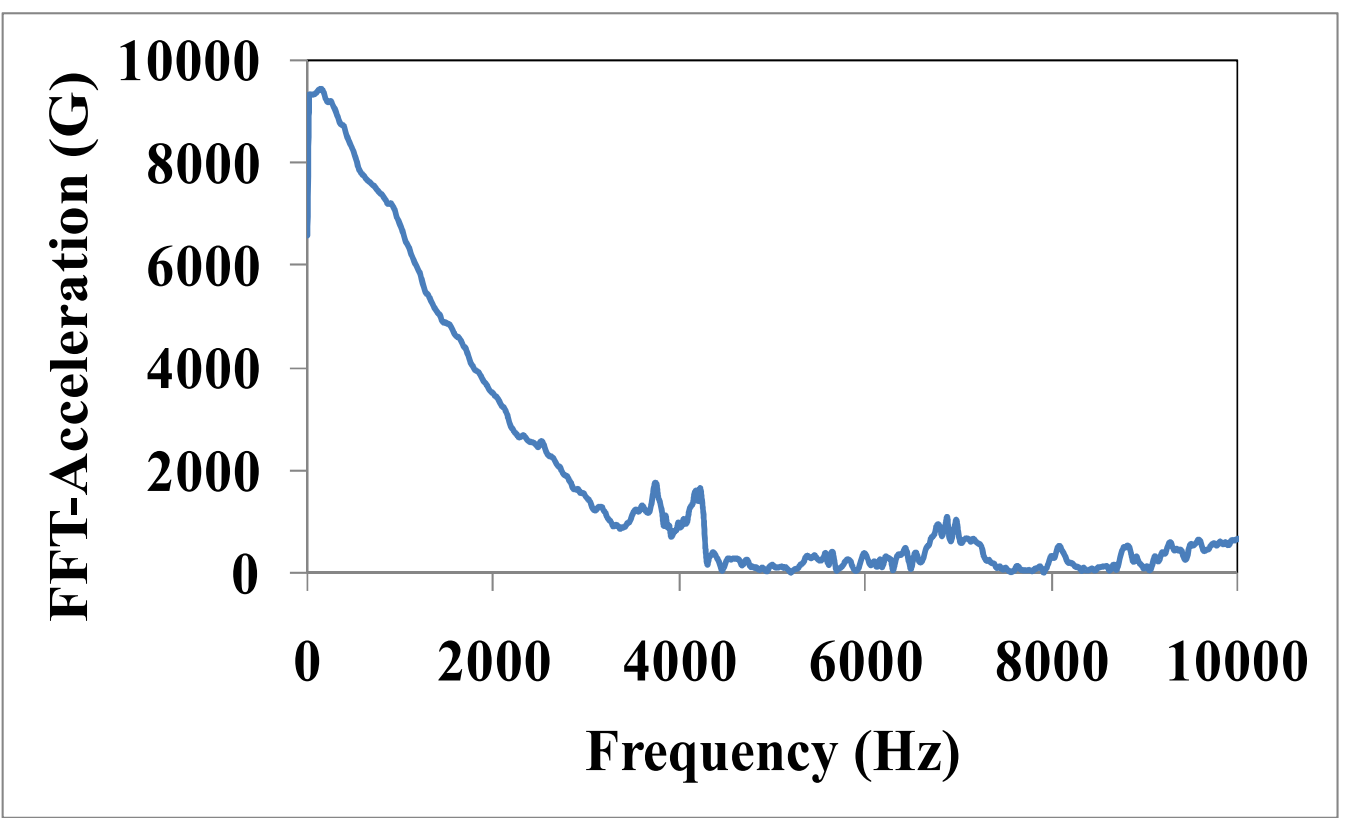

Figure 2-41 FFT response of the drop table fixture to the half sine drop loading

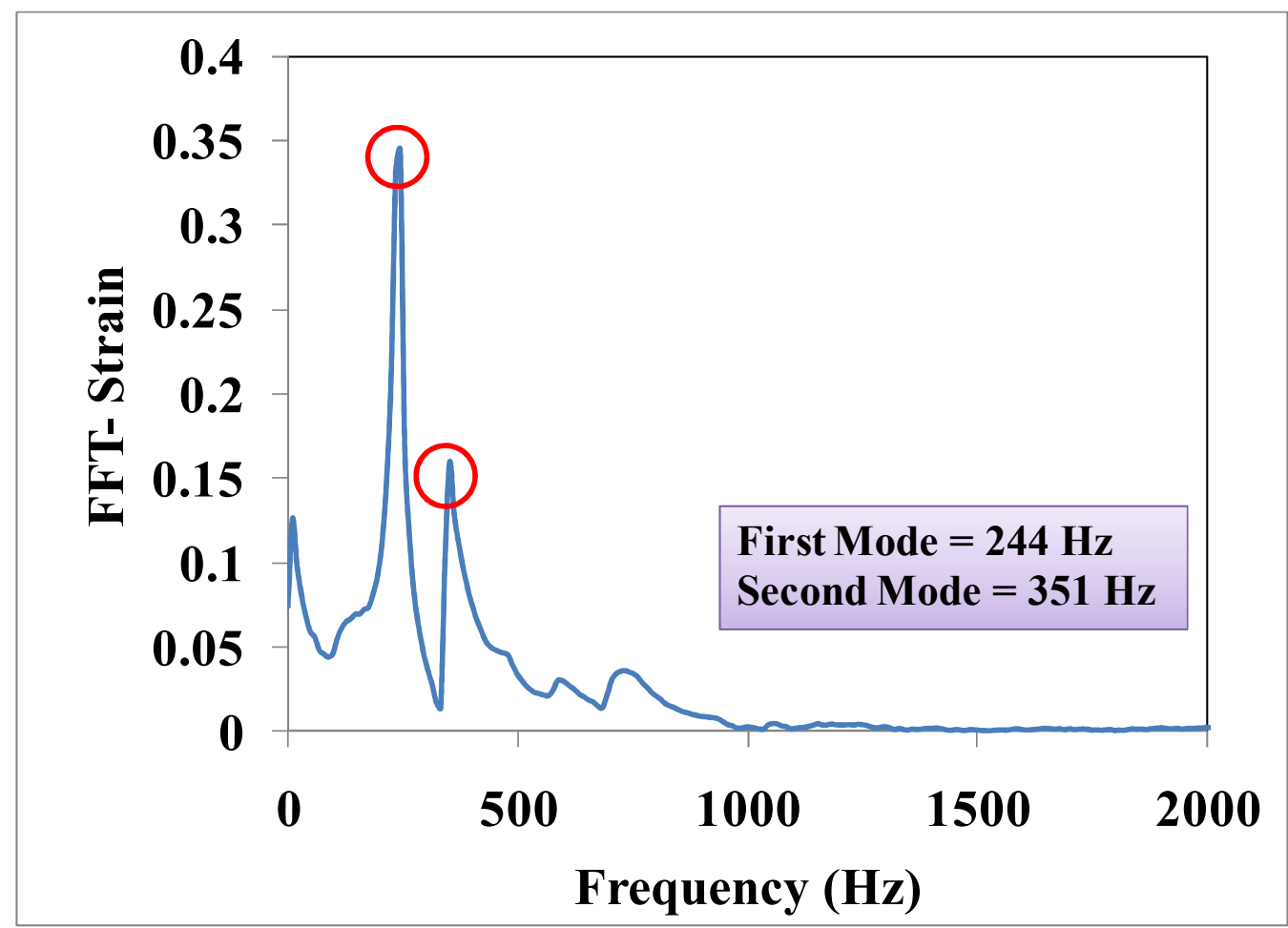

Figure 2-42- FFT response of strain gage in Figure 2-40 to half sine drop loading of clamped PWB 


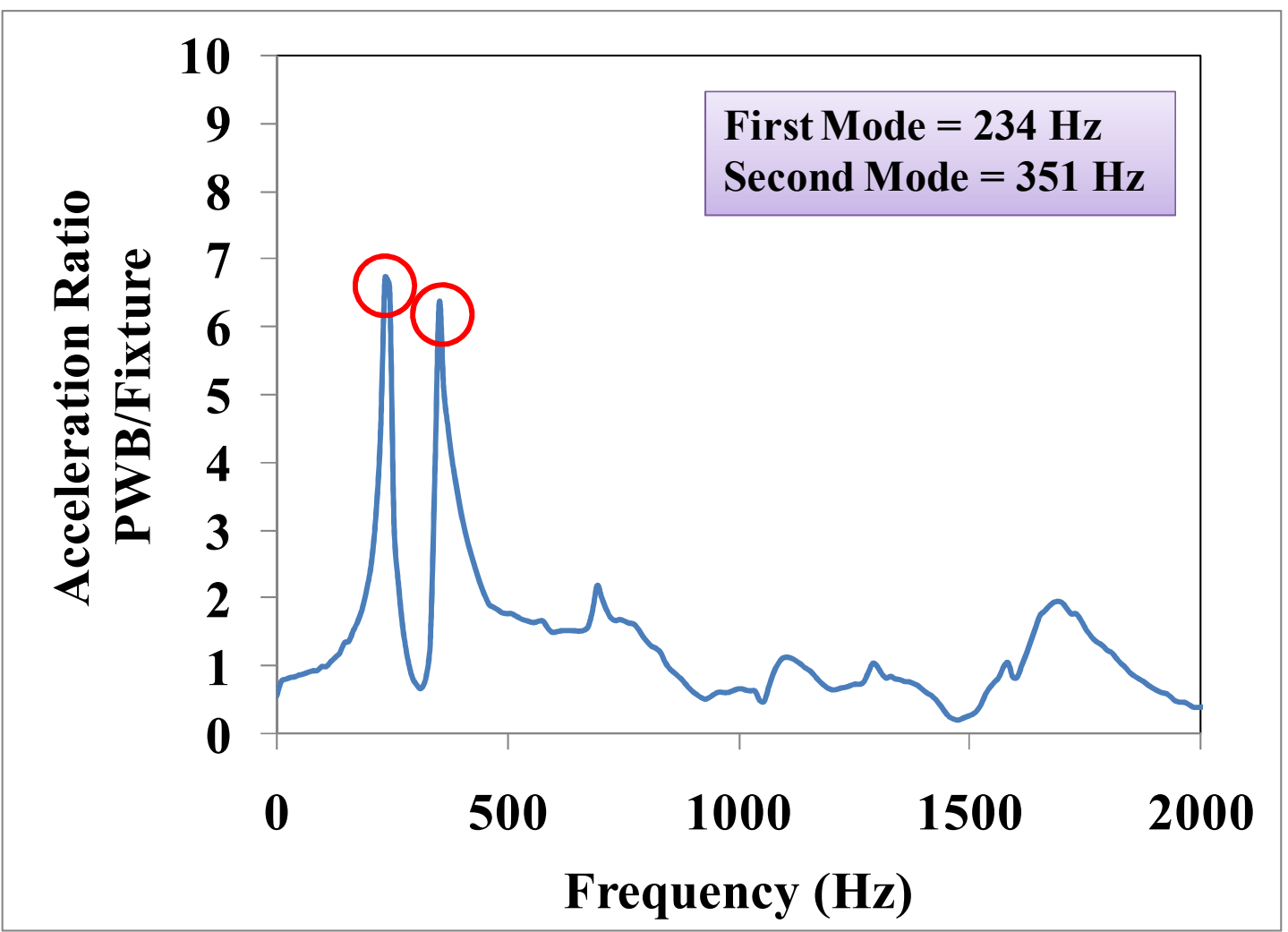

Figure 2-43 Frequency response function of the accelerometer on the free end of the PWB as shown in Figure 2-7.

\subsubsection{Full Product Drop Test Results for Full Product Assembly}

In drop test for the full product assembly, the test specimen is mounted to the drop table, as discussed earlier in Section 2.4.1 and dropped from various heights to determine the relationships between fixture acceleration and PWB acceleration, as well as PWB strain. The locations of the acceleration and strain sensors are described in Figure 2-18 in Section 2.4.1. The correlations of the peak values of these variables are expressed in Figure 2-44 and Figure 2-45, respectively. Linear regression lines are found for each set of data to express the transfer functions between the peak values of these variables. The fixture acceleration will be used later as an input impulse for the modeling in Part II of this study. Acceleration and strain profiles will also be used to compare the simulation and experimental results. To ensure repeatability, each test is 
repeated several times. Each point in Figure 2-44 and Figure 2-45 represents one trial. It is evident that the drop test repeatability decreases as fixture acceleration is increased above 2000 Gs.

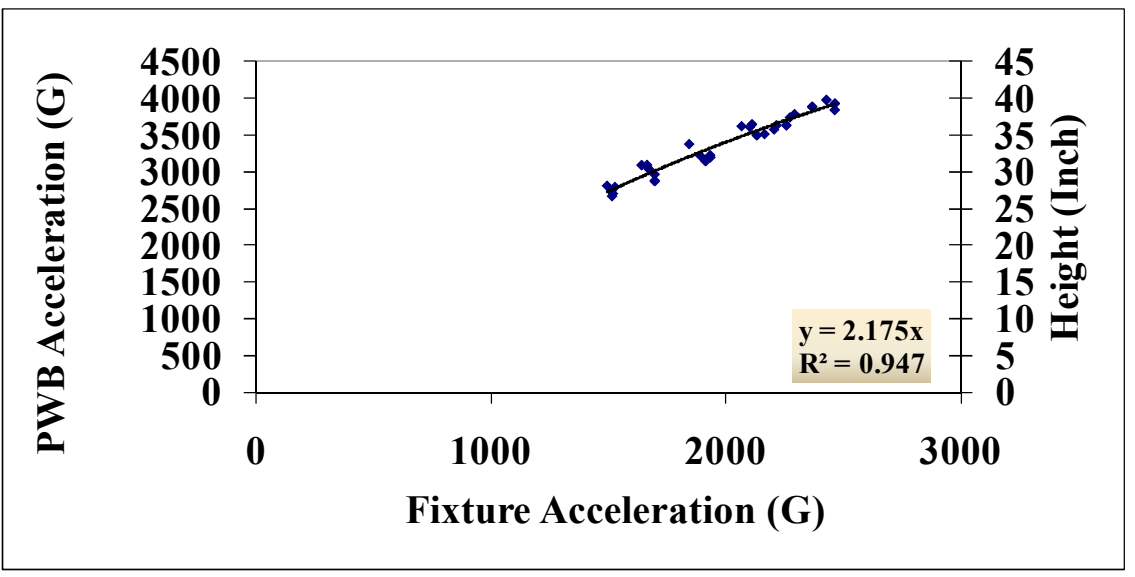

Figure 2-44 Transfer function between peak values of PWB acceleration and fixture acceleration in drop test of full product assembly

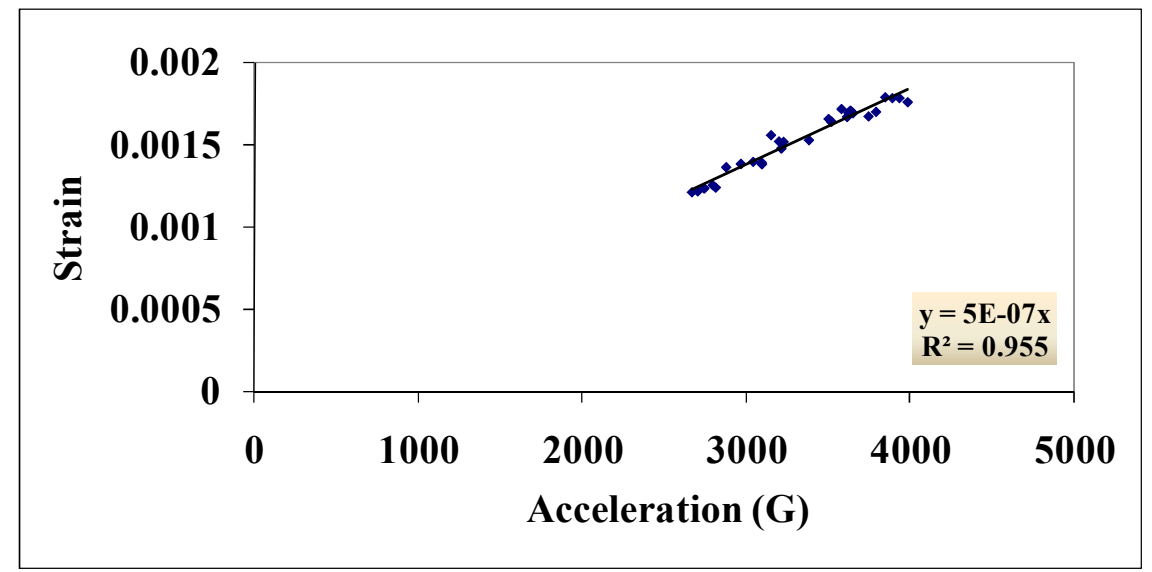

Figure 2-45 Transfer Function between Peak Values of PWB Strain and PWB Acceleration

Figure 2-46 and Figure 2-47 demonstrate acceleration and strain responses of the PWB mounted in the product housing, to $1500 \mathrm{G}$ fixture acceleration. The sensor locations are same as described earlier in Figure 2-18 in Section 2.4.1. Acceleration magnification factor for the case shown below is approximately 1.8 , which is much 
higher than in the shock test. Furthermore, strain at point 2 on the PWB (shown in Figure 2-47) is approximately 1400 microstrains.

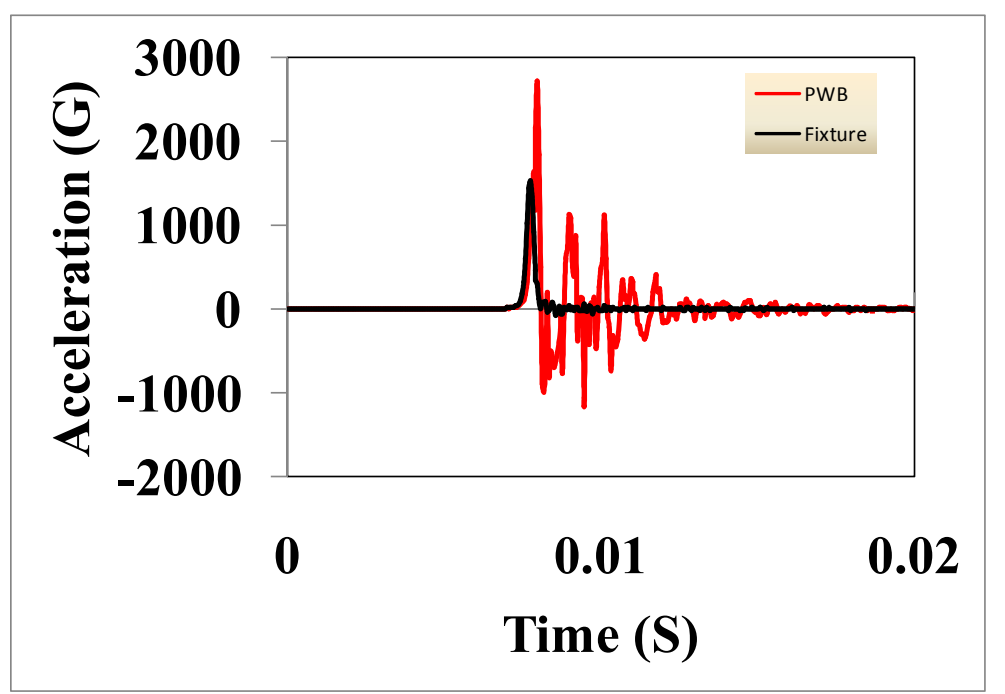

Figure 2-46- PWB Acceleration Vs Fixture Acceleration in Product Drop Test. The exact locations of the accelerometer are shown in Figure 2-18

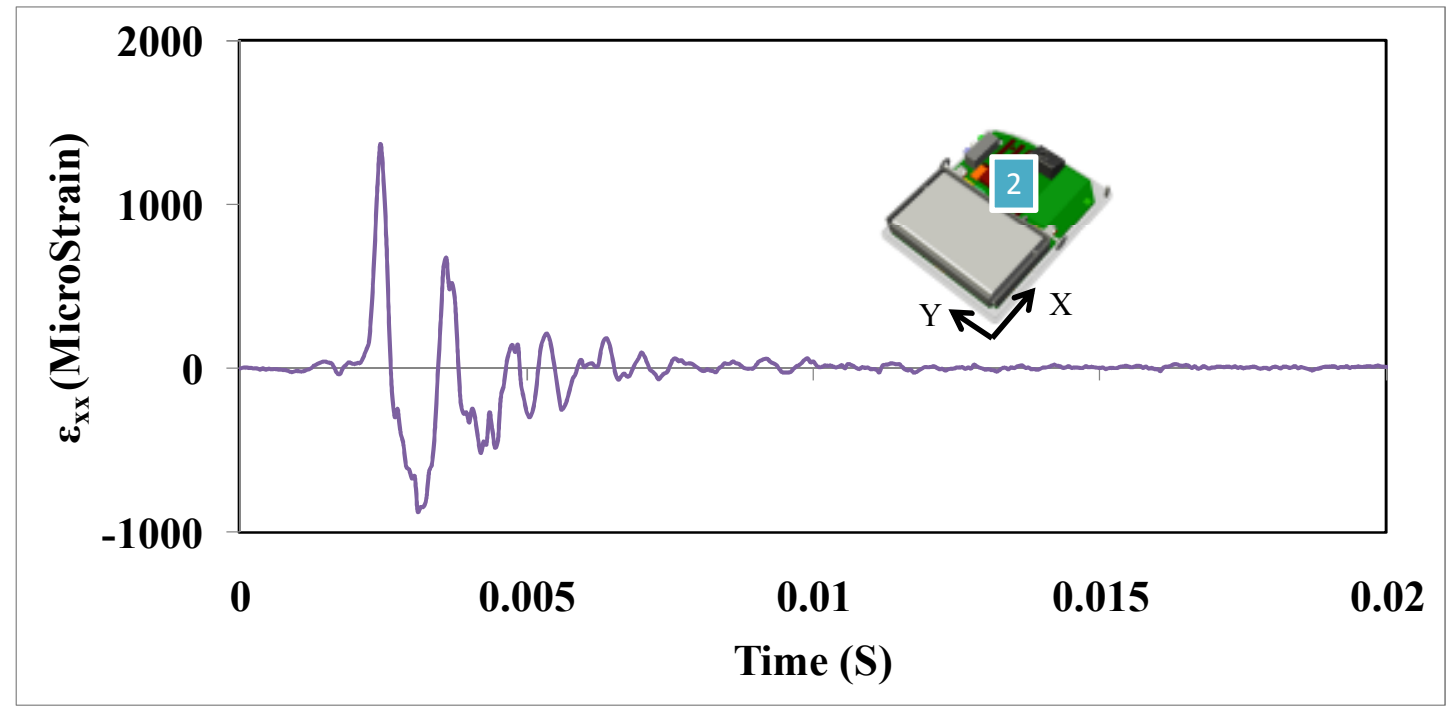

Figure 2-47 PWB strain Response at point 2 in product drop test

Even though the drop test of the full product is conducted at various heights, for simplicity purposes, Part II of this study will only focus on the $1500 \mathrm{G}$ fixture acceleration level. In order to draw accurate comparison between the simulation and experimental results in part II of the study, it is crucial to first study the variations in 
acceleration and strain response of the drop test of the full product. Figure 2-48 illustrates the strain response measured in the " $\mathrm{x}$ " direction, as shown in Figure 2-47, for 5 different trials. There seem to be a bump followed by a valley at the beginning of the strain profiles of the trials $2-5$. The first trial is the only one that does not follow the same trend at the beginning. Therefore, it can be concluded that this is a wire issue that occurred after the first run. The wire problem, however, seem to have insignificant effect on the subsequent peaks. The minimum and maximum values of the first peak amplitude are 1365 microstrain and 1539 microstrain respectively. The deviation in strain profiles beyond the first peak is negligible.

Similarly, Figure 2-49 illustrates the repeatability of the PWB acceleration, measured at the location shown in Figure 2-18, for five different trials. The first peaks of all five trials fall on top of each other. Furthermore, there is no significant deviation among the acceleration profiles beyond the first peak.

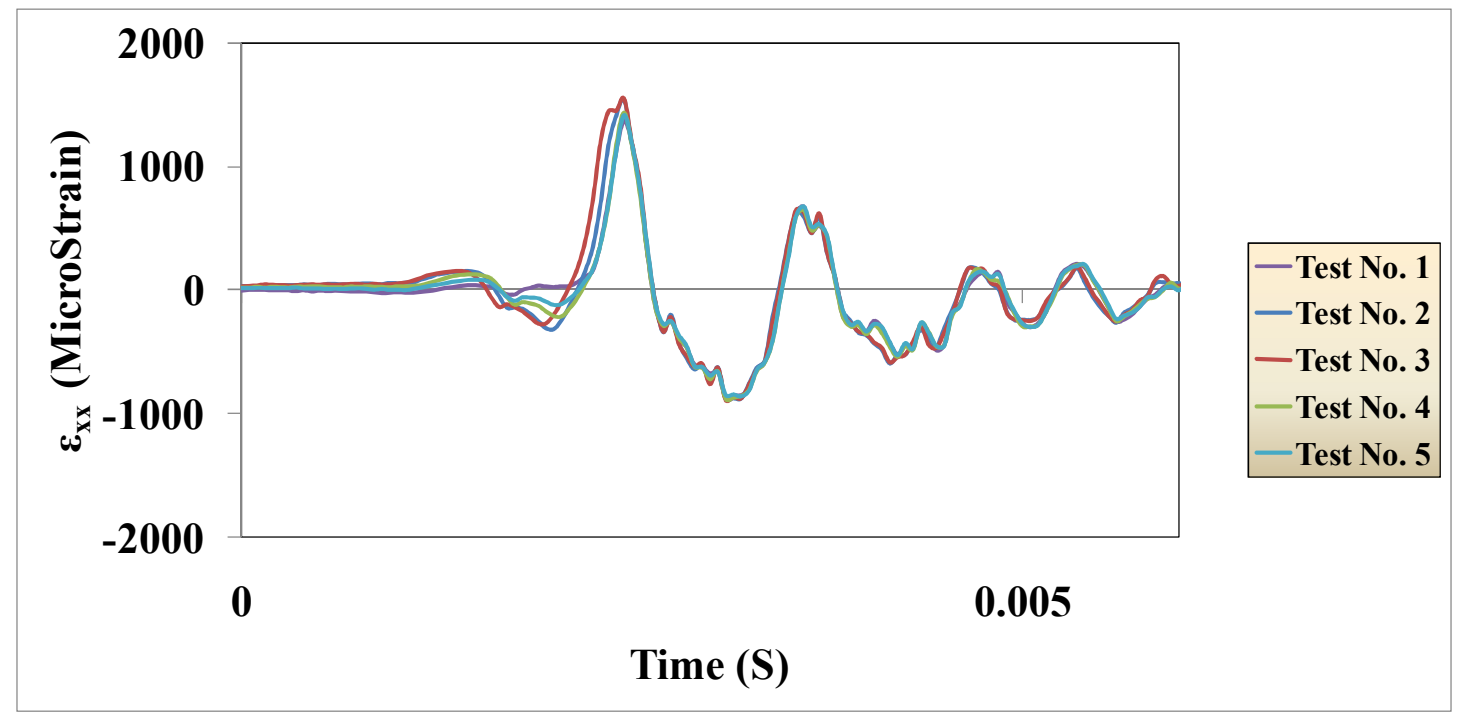

Figure 2-48 Illustrates the repeatability of the PWB strain response measured at point 2 as shown in Figure 2-47 for 5 different trials. Strain is measured in the $\mathrm{xx}$ direction. 


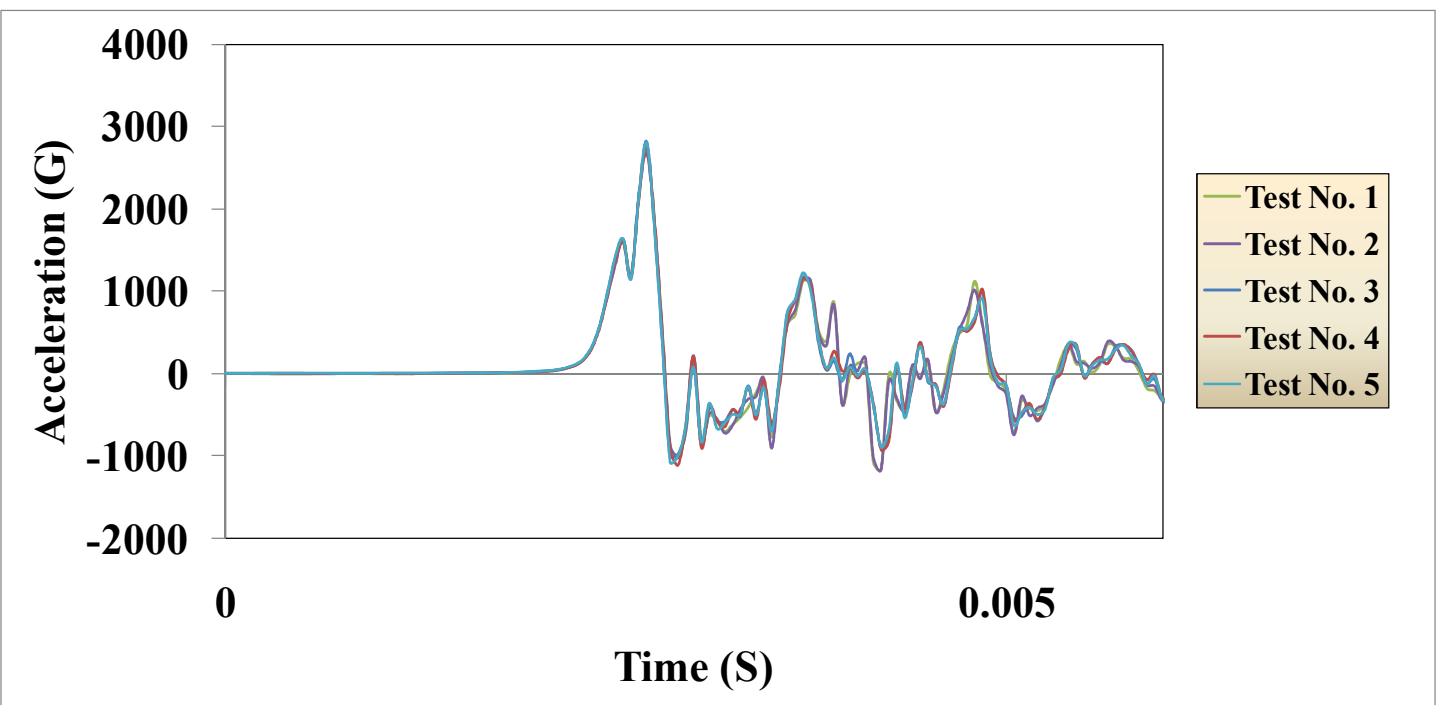

Figure 2-49 Illustrates the repeatability of the PWB acceleration response measured at point 2 as shown in Figure 2-47 for 5 different trails.

Figure 2-50 demonstrates the FFT response of the strain gage on the PWB, as well as the acceleration frequency response function of the PWB. Refer to section 2.3.4 for sensor locations. The first peak of the strain FFT response occurs at $782 \mathrm{~Hz}$, which is relatively close to that of obtained in the broad band test of the full product, $812 \mathrm{~Hz}$ (Refer to Figure 2-33). Furthermore, it seems that the second natural frequency is combined with the first peak. And for that reason, it does not appear in the strain FFT response. This could be due to low frequency resolution of the FFT graph $( \pm 40 \mathrm{~Hz})$. There is a very good match among the third $(1330 \mathrm{~Hz})$ and forth $(1448 \mathrm{~Hz})$ natural modes of the strain FFT response of the strain gage to drop loading to that of obtained in the broad band test of the full product, $1356 \mathrm{~Hz}$ and $1400 \mathrm{~Hz}$ respectively. Unlike the FFT response of the strain gage, the second natural mode obtained from the acceleration frequency response appears in the graph $(1071 \mathrm{~Hz})$ and the first natural frequency peak is combined with the second excitation peak. This is mainly due to that fact that acceleration and strain are measured at two different locations. Therefore, it can be concluded that the first mode shape of the system is 
more prominent at the location of the strain gage and vice versa. As explained above, response frequencies of the PWB in the full product match reasonably well in both broad band and drop tests, within the measurement resolution $( \pm 40 \mathrm{~Hz})$. Generally, the frequency response of a non-linear system is expected to change with the level and type of loading. In this case, the effects of non-linearities do not appear to be strong enough to be detectable with our measurement resolution.

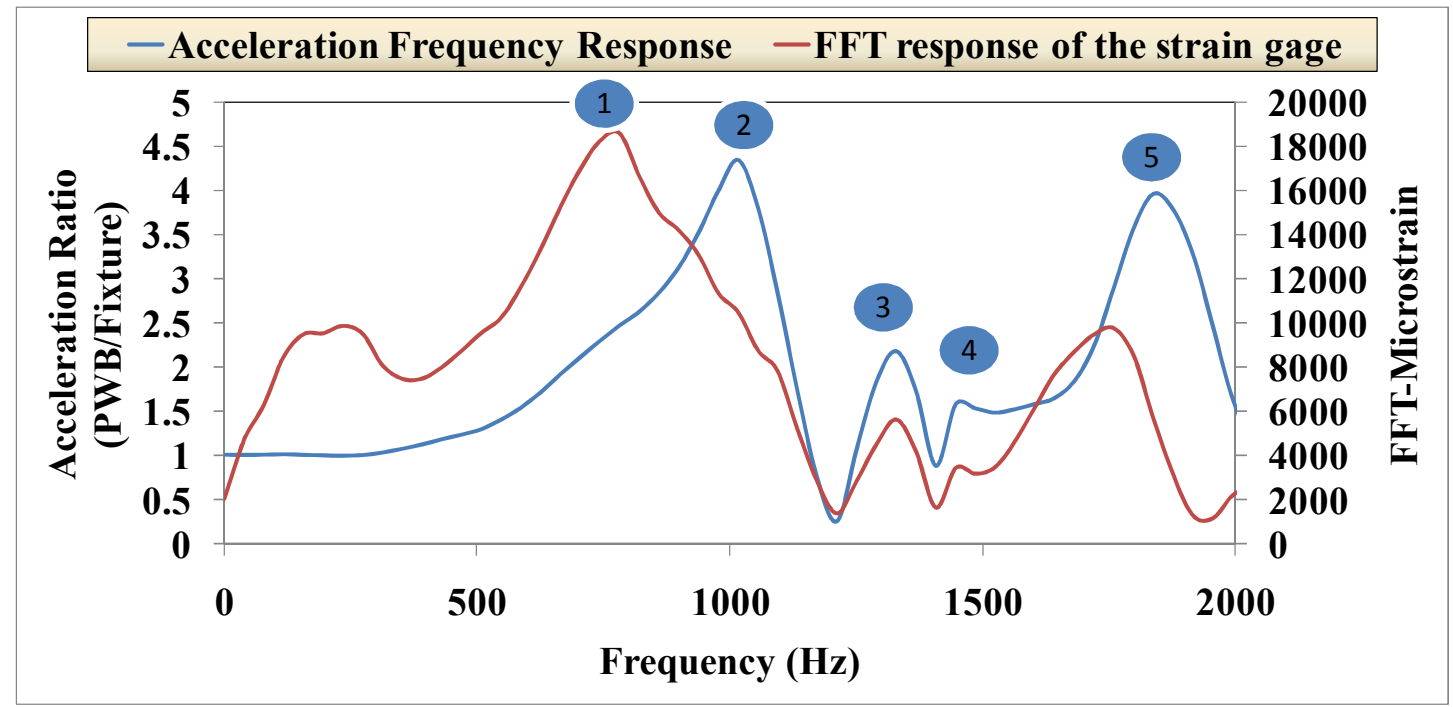

Figure 2-50 FFT response of the strain gage on the PWB, shown in Figure 2-47, to drop loading. Acceleration frequency response of the PWB measured at the location shown in Figure 2-18.

\section{$\underline{\text { 2.6 Summary }}$}

The ultimate goal of this paper is to develop a systematic modeling approach that is capable of capturing the dynamic response of reasonably complex portable electronic devices. This part of the study has presented a comprehensive experimental understanding of the dynamic behavior of the product under study and its subassemblies. A combination of shock, random vibration, and drop tests were conducted both on the full product and on its constituent components. The input accelerations measured in this part of the study will be used as boundary conditions later in the finite element modeling reported in Part II of the study. Additionally, in 
Part II of the study, the modeling results will be calibrated with respect to the experimental response measurements (accelerations and strains) reported in this paper. This research highlights the importance of first understanding the dynamic response of the fixture on which the test vehicle is mounted, before running the actual experiment. Failing to do so could result in inaccurate results. Furthermore, the importance of determining the dominant mode of the system is demonstrated. Furthermore, it was shown that the dominant natural mode of a freely vibrating system is decided by the zeros and peaks of the spectral content of the input pulse. The zeros of the input pulse are a function of pulse width. Our results also indicate that shock tests performed are more repeatable than drop tests. Another observation that was made was that the PWB acceleration magnification factor in product level shock test reduces to 1.15 from 1.4 in board level shock test. This is mainly due to absorbance of some of the energy by the plastic housing in product level shock test. Conversely, in product level drop test, the PWB acceleration magnification factor increased in comparison to that of PWB-level drop test. It is believed that internal components of the device impact against each other due to high accelerations generated in drop test which leads to a higher acceleration magnification factor. 


\section{Chapter 3 Modeling For Shock and Dynamic Loading in Portable Electronic Products}

The text of this chapter is taken from a paper that has been submitted for publication. This chapter is Part II of a two-part study. The first part was presented in Chapter 2 of this thesis. The aim of this chapter is to investigate the accuracy of computational models in predicting the transient response of complex portable electronic assemblies subjected to shock and drop loading. The problem is challenging because the many nonlinearities and uncertainties in such problems make it very difficult to find the root-cause of discrepancies between simulation and experimental results. In order to make the problem tractable, in this study the full assembly is divided into its constituent subsystems. Additionally, to isolate some of the nonlinearities involved in drop conditions, the response of the system and its subassemblies to half-sine shock loading and random vibration is first studied. Finite Element models for shock and random vibration tests are used to calibrate the material properties and boundary conditions for these less nonlinear conditions before performing Part I of this study, presented in Chapter 2, experimental results for random vibration, shock, and drop tests were presented. In this chapter, the input loadings that were used in the tests are applied as the boundary conditions to the finite element models. Furthermore, the test and simulation results are compared with each other, both in time and frequency domains, to calibrate and validate the finite element models. Our results indicate that performing modal analysis prior to shock and drop simulations is very crucial. Finding the natural frequencies and dynamic response

amplitudes of a system can greatly assist in providing accurate estimates for the 
modulus of elasticity as well as damping ratios. Good agreement is found between experimental and simulation results in shock and drop modeling of the subassemblies of the system. In the case of the full product a comprehensive parametric study is conducted on various mechanical contact property options available to roughly simulate the effect of plastic deformation. In this paper, random vibration and shock modeling results are presented first and followed by the drop modeling results.

\title{
SHOCK AND DYNAMIC LOADING IN PORTABLE ELECTRONIC ASSEMBLIES: PART II- MODELING \& SIMULATION RESULTS
}

\author{
Alex. F Askari Farahani, Moustafa Al-Bassyiouni, Abhijit Dasgupta (Corresponding \\ Author) \\ CALCE Electronic Products and Systems Center \\ Mechanical Engineering Department \\ University of Maryland, College Park, MD 20742 USA \\ Sheldon Tolchinsky, Jack Crystal \\ Arbitron INC. \\ 9705 Patuxent Woods Drive, Columbia, Maryland 21046
}

\section{Abstract}

In this study, the transient response of electronic assemblies to mechanical loading encountered in drop and shock conditions are investigated through modeling and simulation. The development of portable electronics poses design challenges when evolving new designs for high strain-rate life-cycle loading, such as in drop events, blast events, vibration, ultrasonic process steps, etc. Electronics designers have long sought to address these effects during the design phase, with the aid of computational models. However, such efforts have been difficult because of the uncertainties and inaccuracies caused by nonlinearities inherent in complex assemblies and by complex dynamic material properties. Our goal in this study is to 
investigate the ability of finite element models to accurately capture the transient response of a complex portable electronic product under shock and drop loading. The product consists of a circuit card assembly and a battery pack enclosed in a welded plastic housing. While many researchers have shown qualitative ability for such modeling, further work is still needed to demonstrate good quantitative agreement. This paper is the second portion of a two part study. In Part I, broad-band modal tests as well as shock and drop tests were conducted on the assembly as well as on its subassemblies, using an electrodynamic shaker and a drop tower.

In this paper, the focus is on simulating the tests that were run in Part I. The modeling is conducted in ABAQUSTM [16]. Flexural strains and accelerations are compared to assess the agreement between the model results obtained here and the experimental results reported earlier in Part I of this study. The long-term goal of this study is to demonstrate a systematic computational capability to predict the expected dynamic response and failure modes during the design phase of future products.

\section{$\underline{3.1 \text { Introduction }}$}

Designers have long realized the benefits of effective modeling and simulation during the design cycle. In particular, during the last decade, designers of portable electronic systems have explored the effective use of transient finite element modeling strategies for design assurance of products that are subjected to life cycles that include high-strain-rate loading conditions. Examples of high- strain rate loading include events such as drop, blast, shock, impact, vibration, ultrasonic processing 
steps, etc. The goal is to use the model results to perform early design iterations so as to anticipate and minimize costly trial-and-error empirical design-fixes later during qualification testing or after the product design has been finalized. The advantage of such a modeling capability is clear, because of its importance in developing design guidelines, in timely virtual qualification of new design concepts, and in helping extrapolate results of accelerated testing to field conditions.

Currently, engineers mostly use finite element models to study the local effects of drop loading, under the assumption of simple deformation at the contact areas at the time of impact [7]. As mentioned earlier, even though system-level modeling could be very advantageous during product development, further research is needed to simulate the impact response of the components and assemblies located in a portable electronic product subjected to high-strain rate loading conditions to identify any potential failure modes [8]. One of the main reasons is that system-level modeling is extremely challenging due to nonlinear effects such as contact stresses, large deformation, and complex dynamic material properties. Besides computational modeling, analytical models have been proposed as well. Goyal [7] modeled the facedown impact of a cellular phone with a linear spring mass system. He used beam theory to demonstrate the importance of structurally connecting the battery to the rest of the device, to minimize deflection at the top of the cellular phones. He also concluded that the geometry of the product plays an important role in drop survivability of the system and suggested various techniques to improve the ruggedness of hand-held electronic devices. Additionally, other studies [3][9] have confirmed that different drop orientations from the same height produce noticeably 
different acceleration levels. The acceleration experienced by the product largely depends on contact area at the time of impact. Irving [10] investigated the free drop performance of portable IC package, using an implicit solver to simulate the whole drop event from the release of the test vehicle to its final rest state after the impact. Scott Irving did not, however, provide any empirical results to validate the simulation results. Since it is tremendously difficult to define the correct surface contact and felt material properties, Luan [11] used an input G method to perform board level drop simulation. In input $\mathrm{G}$ method, an accelerometer is placed on the fixture and the accelerometer measurements are used as a boundary condition in drop modeling. Luan demonstrated that simulation results of the input $\mathrm{G}$ method for implicit transient analysis of a board correlate well to experimental results. This is particularly a good alternative solution for organizations that do not have access to explicit solvers [11]. Liu [12] also compared the measured drop response of a cell phone to simulation results, but the agreement there was poor. He concluded that the simplifications that were made in the geometry of the cell phone were the main reasons for the inconsistencies. To detect failure of the small internal components in electronic devices due to drop, one must rely on the simulation results of the whole device model [13]. This requires very fine mesh at the location of the small components which causes very long simulation times. As a result, $\mathrm{Wu}$ [13] proposed a global and local coupled analysis to detect failure in small electronic components. In this method, $\mathrm{Wu}$ replaces the solder joints and small electronic components with spring elements and then performs global analysis on a coarsely meshed model of the whole device. The simulation output of the global model is then used as a boundary 
condition to perform local analysis on a finely meshed solder joint. This study, however, was conducted solely to better understand and overcome the difficulties of component level analysis and no empirical results were provided to confirm the accuracy of the computational models.

The present study utilizes a combination of simple shock and drop tests on the full assembly, as well as on the main components and sub-assemblies, such as printed wiring board (PWB), spring contacts, and plastic housing. Finite element models are also developed for each phase of the drop and shock tests, and calibrated with the test results. The goal is to isolate some of the nonlinearities involved in drop tests by performing simple shock tests and to investigate whether or not the calibrated finite element models for simple shock tests or sub-assemblies can be used for drop test simulations of the final assembly.

\subsection{Test Specimen}

The test specimen has been described earlier in Part I of this study, and is summarized again here for completeness. The device in this study consists of a PWB which has three LEDs, a microphone, and its housing, as well as two Au-plated leaf springs mounted on it. The PWB sits inside a plastic housing on several ribs that are located along the walls of the top and bottom housings. It is also seated on the battery case of the plastic housing. The PWB is also held in place by two star-shaped tapered posts, one fixed on the bottom housing and the other is fixed on the battery case. 


\subsection{Broad Band and Shock Modeling Results}

Section 3.3 discusses Shock and broad band modeling results of all the subassemblies of the device under study as well as the full device. Modeling results are calibrated with respect to the experimental results obtained in the part I of this study.

\subsubsection{Finite Element Modeling of Clamped PWB}

The Newton-Millimeter-Ton-Second unit system is used for the finite element analysis. The main advantage of this unit system is that it can analyze sub-millimeter dimensions and deflections when the system is subjected to high force amplitudes, without losing accuracy because of the numerical round-off errors. This unit system is used throughout this paper for modeling of other components of the device as well as the full product under the study. The models created for modal and shock simulations of the bare board are very similar to each other. They only differ in boundary conditions applied to the models. Therefore, the simulation models for modal analysis and shock response of the clamped PWB are explained in this section together. The differences in boundary conditions are explained wherever necessary. Four-node, quadrilateral, stress/displacement shell elements with large-strain formulation are used to create the geometry of the bare board. The use of shell elements reduces the number of nodes and elements in the model and, hence, lowers simulation time. Post holes one and two, shown in Figure 3-1, are fixed in all directions for modal analysis to find the natural frequencies of the PWB. When modeling the shock response of the $\mathrm{PWB}$, a rigid constraint is applied to post holes one and two since they move together on the fixture during the shock tests. A reference point needs to be defined for the 
rigid constraint, as shown in Figure 3-1. The output of the accelerometer on the fixture is applied as a boundary condition to the reference point assigned to the rigid constraint for shock simulations. Accelerometer and LEDs masses are added to the model as point and distributed masses, respectively, at their designated locations, as shown in Figure 3-1. The PWB material defined in this analysis is FR4 and it is modeled as an orthotropic materials. FR4 material properties are obtained from literature [19] and are listed in Appendix B.

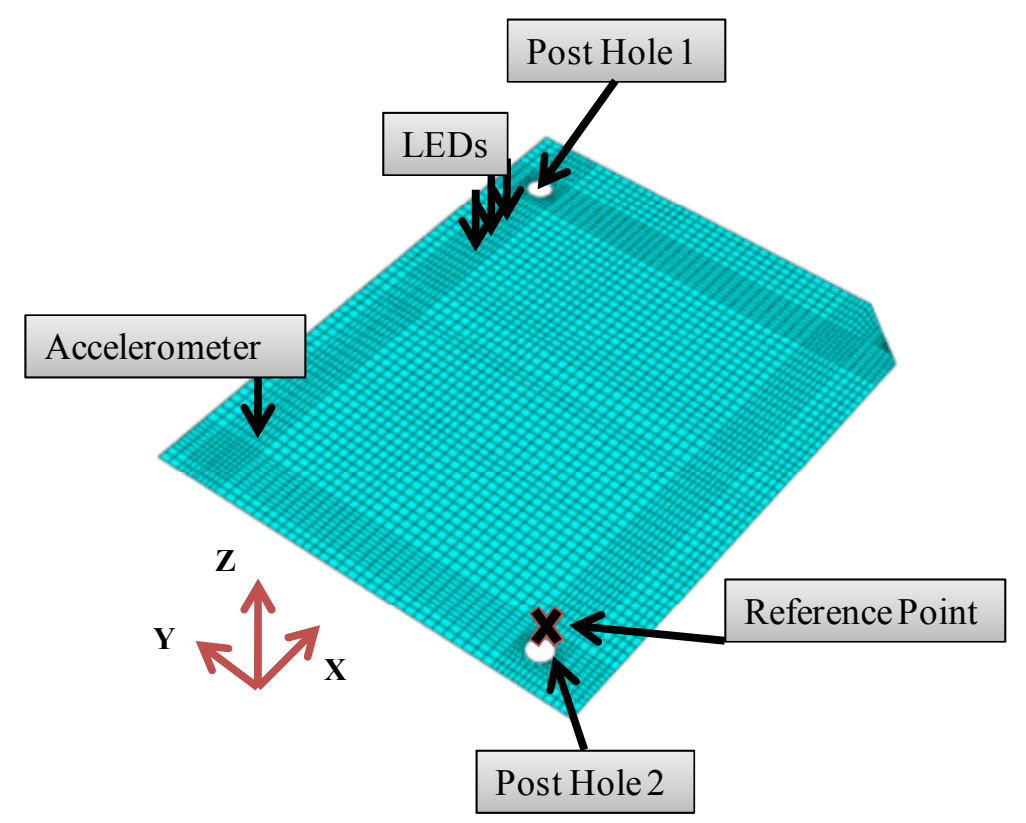

Figure 3-1- Clamped PWB meshed geometry. The PWB is fixed at points 1 and 2. Accelerometer and LEDs masses are added at their designated locations.

ABAQUS/Standard ${ }^{\mathrm{TM}}$ is used to conduct an eigenvalue extraction, in order to calculate the natural frequencies and the corresponding mode shapes of the system. As expected, the first two natural frequencies obtained from simulation, based on the FR4 properties [19], are lower than the natural frequencies obtained from the experiment, due to the stiffening effect of copper traces in the PWB. A $20 \%$ increase in the modulus of elasticity appears to satisfactorily account for this stiffening effect, 
since the two resulting natural frequencies $(229 \mathrm{~Hz}$ and $347 \mathrm{~Hz}$, respectively) now agree reasonably well with the experimental measurements $(231 \mathrm{~Hz}, 354 \mathrm{~Hz})$. The corresponding mode shapes are shown in Figure 3-2.

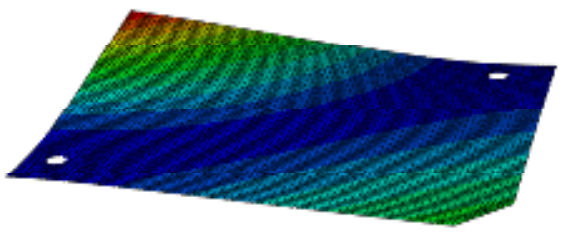

Mode $1229 \mathrm{~Hz}$

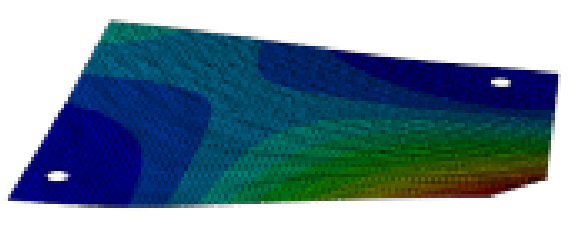

Mode $2347 \mathrm{~Hz}$

Figure 3-2- The first two mode shapes of the clamped PWB with $20 \%$ increase in elastic modulus

The modified material properties of the PWB are listed in Appendix B, Figure B.2. Another important piece of information that can be obtained from the results of broad band test is the damping ratio $\xi_{\mathrm{i}}$. To find $\xi_{\mathrm{i}}$ values, a mode-based steady-state dynamic step must be defined in ABAQUS/Standard ${ }^{\mathrm{TM}}$. This step calculates the steady-state dynamic linearized response of a system to harmonic excitation. This is accomplished by calculating the response based on the system's eigenfrequencies and modes obtained in the previous step explained above. A harmonic excitation with constant amplitude of $1 \mathrm{G}$ in the vertical direction is defined as a base motion for the model. Initially $\xi_{\mathrm{i}}$ values are set to zero for each mode and then simulation is run to acquire the frequency response of the system. As predicted, acceleration amplitudes at first and second natural frequencies increase without bounds. $\xi_{\mathrm{i}}$ values are then calibrated for each mode $\left(\xi_{1}=0.041\right.$ and $\left.\xi_{2}=0.0087\right)$ until acceleration amplitudes predicted by FEA simulation for first and second modes matched those measured in experiments, as shown in Figure 3-3. 
ABAQUS/Explicit ${ }^{\mathrm{TM}}$ is next used to perform shock simulation on the clamped PWB, because it is computationally efficient for the transient analysis of large models with relatively short dynamic response times. To perform dynamic explicit analysis, Rayleigh damping must be defined rather than the fraction of critical damping, $\xi_{i}$. Therefore, Rayleigh damping constants must be deduced from $\xi_{\mathrm{i}}$ values, using Equation 3.2. To define Rayleigh damping, two Rayleigh damping factors are needed: $\alpha$ for mass-proportional damping and $\beta$ for stiffness-proportional damping. In general, damping is a material property specified as part of the material definition and defined by Equation 3.1[14]:

$[C]=\alpha[M]+\beta[K]$

Equation 3.1

$[\mathrm{C}]=$ damping matrix of the physical system;

$[\mathrm{M}]=$ mass matrix of the physical system;

$[\mathrm{K}]=$ stiffness matrix of the system;

$\alpha$ and $\beta$ are pre-defined constants. For a given mode $i$ the fraction of critical damping, $\xi_{i}$, can be expressed in terms of the damping factors $\alpha$ and $\beta$ as [14]:

$$
\xi_{i}=\frac{\alpha}{2 \omega_{i}}+\frac{\beta \omega_{i}}{2}
$$

Equation 3.2

In the above equation, $\omega_{i}$ is the natural frequency of the $i^{\text {th }}$ mode. This equation implies that, generally speaking, the mass-proportional Rayleigh damping, 
$\alpha$, damps the lower frequencies and the stiffness-proportional Rayleigh damping, $\beta$, damps the higher frequencies.

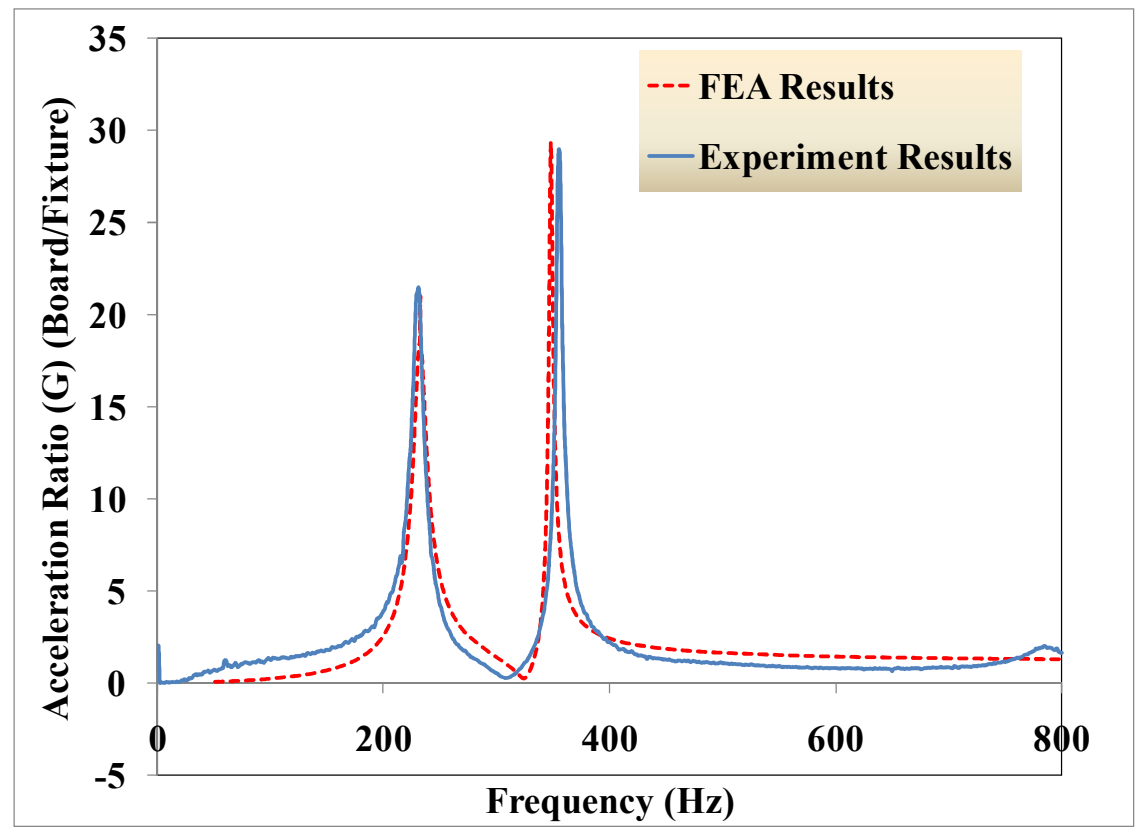

Figure 3-3-Natural Frequencies of the clamped PWB

The use of stiffness-proportional material damping can reduce the stable time increment dramatically and can lead to excessively long simulation times. Therefore $\beta$ is set equal to zero in all explicit analysis in this study. Alternatively, to predict the value of $\alpha$, assuming $\beta=0$, one could directly define separate $\alpha$ values for each mode of the system during modal analysis to obtain a similar graph to that of Figure 3-3. Subsequently, it is necessary to determine the dominant mode of the system and utilize the corresponding $\alpha$ value in dynamic explicit analysis.

For this particular case, $\alpha$ values turned out to be $69 \mathrm{Rad} / \mathrm{s}$ and $19 \mathrm{Rad} / \mathrm{s}$ for first and second modes, respectively. As discussed in Part I of this study, the shock spectrum of the input pulse showed that the electrodynamic shaker does not excite the first mode of the clamped PWB for a $6 \mathrm{~ms}$ pulse duration. As a result, the second 
mode is the dominant mode. Therefore the value of $\alpha$ is set to $19 \mathrm{Rad} / \mathrm{s}$ for the shock simulation of the clamped PWB. Furthermore, the calibrated material properties obtained in the PWB modal analysis (shown in Appendix B) are used in the shock simulations.

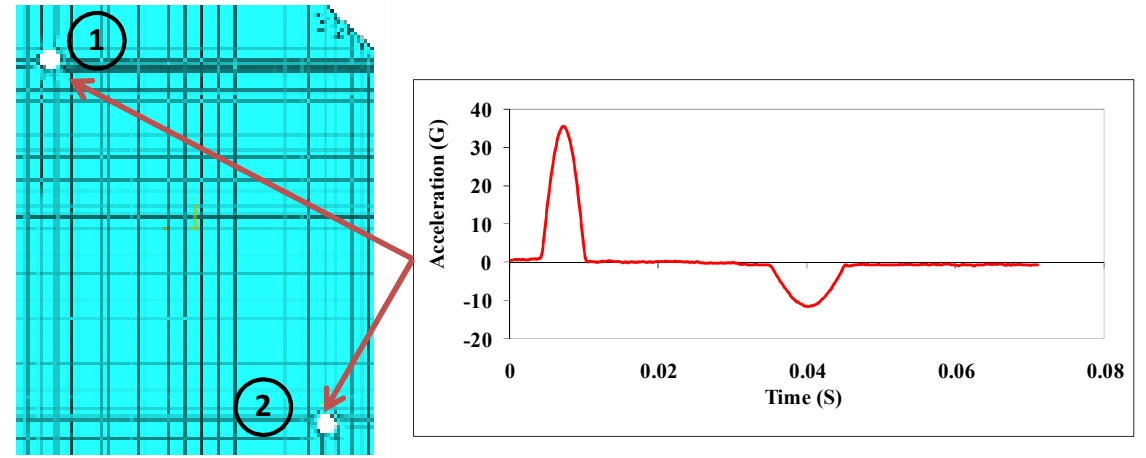

Figure 3-4- FEA input pulse applied at points 1 and 2

Figure 3-4 shows the input pulse that is applied as a boundary condition at post holes $1 \& 2$. Figure 3-5 and Figure 3-6 compare the results obtained from FEA analysis with those from experiment. As shown below, the FEA model is able to accurately capture the dynamic behavior of the board when subjected to a half sine shock test. Predicted values of the maximum acceleration at the free end and the maximum strain at the post match their corresponding measured values. Additionally, both acceleration and strain profiles obtained from the simulation follow the same trend as those obtained from the experimental measurements. 


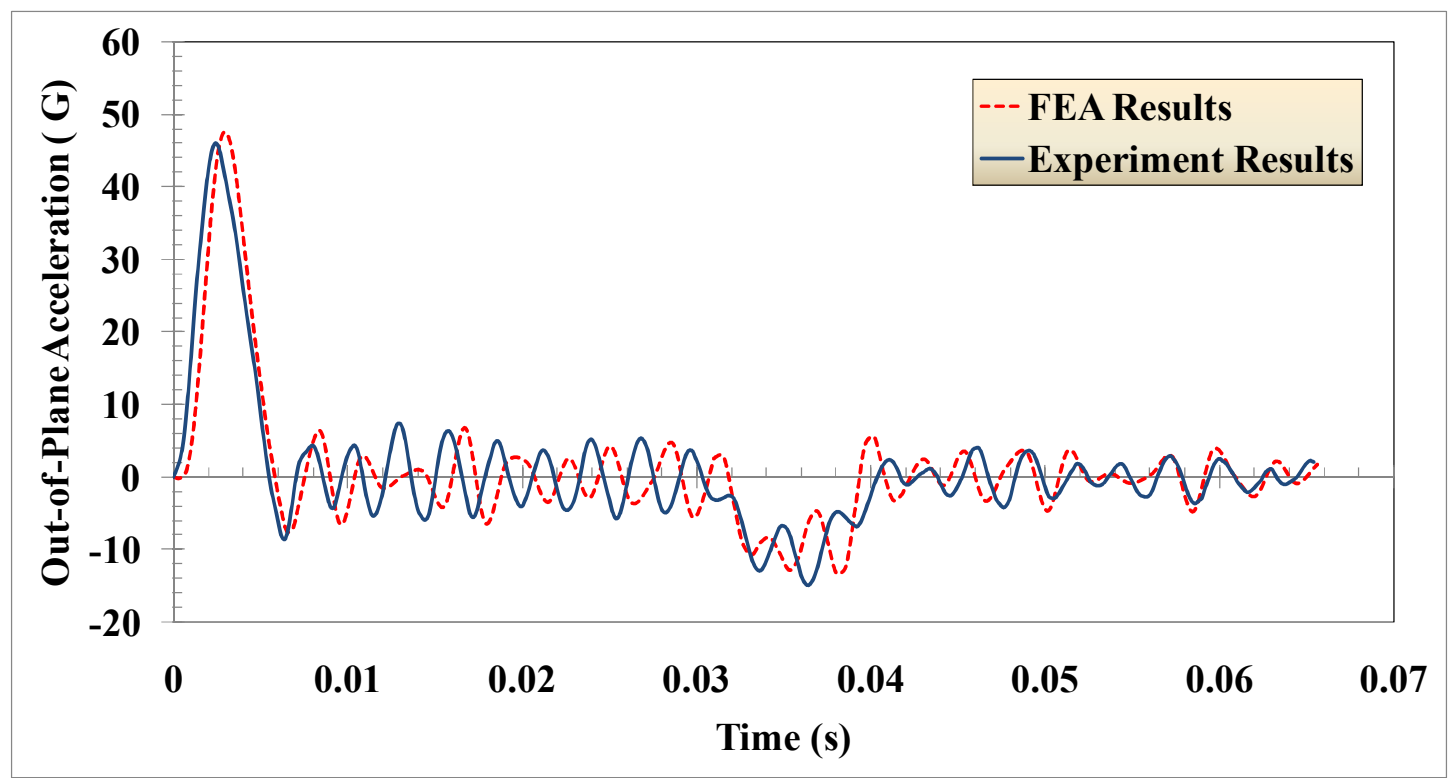

Figure 3-5 Acceleration at the free end of the clamped PWB. Acceleration is measured in the out-of-plane direction- FEA VS. Experiment

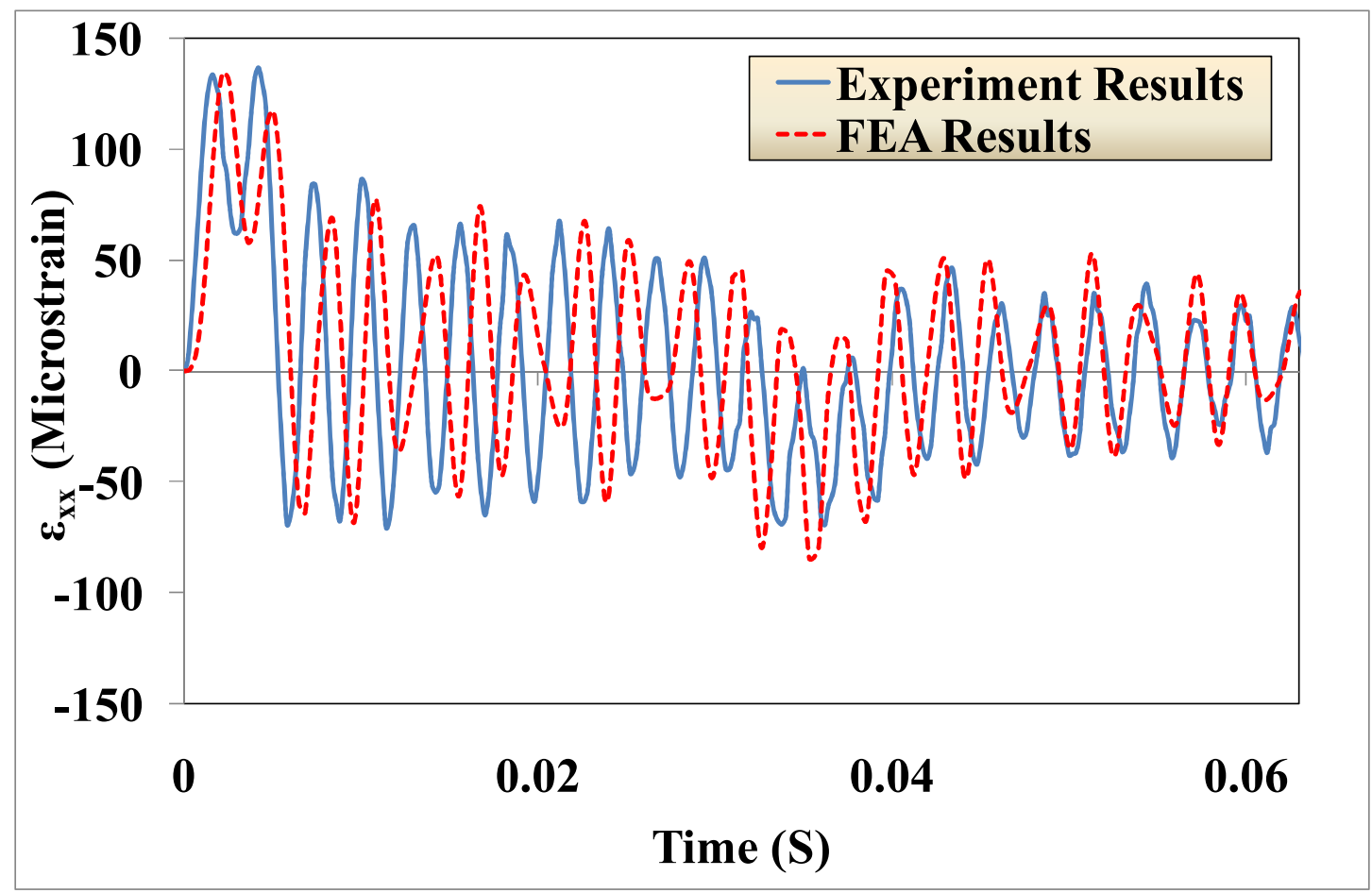

Figure 3-6 Strain at the post of the clamped PWB. Strain is measured in the $\mathbf{x x}$ direction as shown in Figure 2-24- FEA VS. Experiment-

As shown in Figure 3-5 and Figure 3-6, the amplitude of strain and acceleration after the first impulse also agree reasonably well with experimental data. 
This confirms the accuracy of the $\alpha$ value chosen for shock simulations. The good agreement found here between simulation and test emphasizes the importance of performing broad band tests and calibrating material properties before conducting shock simulations. Furthermore, the FFT response of the strain histories are shown in Figure 3-7, confirming that the second mode (occurring at $354 \mathrm{~Hz}$ ) is the dominant mode in the FEA analysis. The disagreement in the amplitude of the FFT histories, apart from the complexity of the system, is possibly due to the low resolution of the FFT graphs $( \pm 20 \mathrm{~Hz})$.

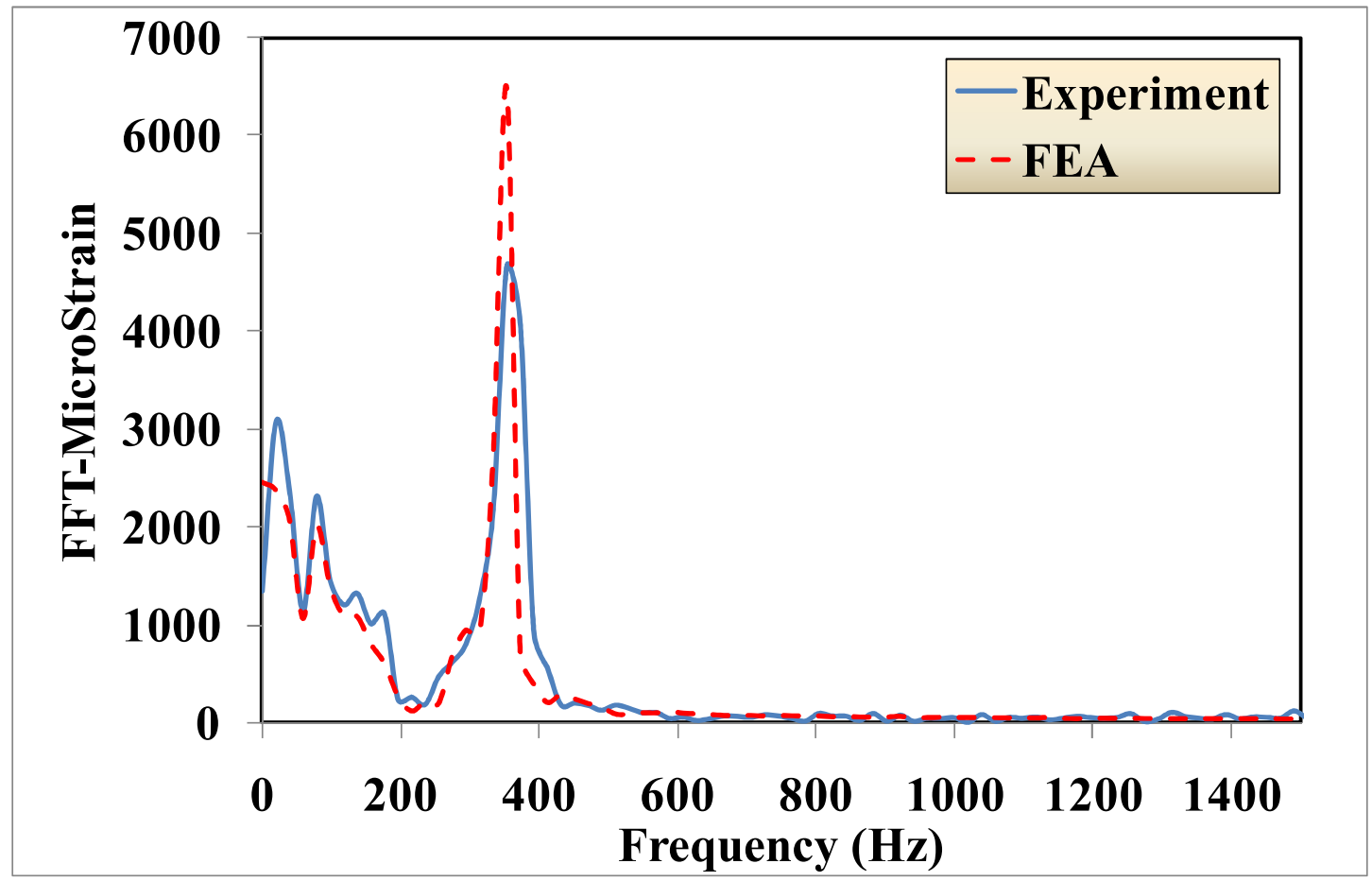

Figure 3-7- FFT response of strain gage mounted at the post of the clamped PWB shown in Figure 2-24FEA VS Experiment 


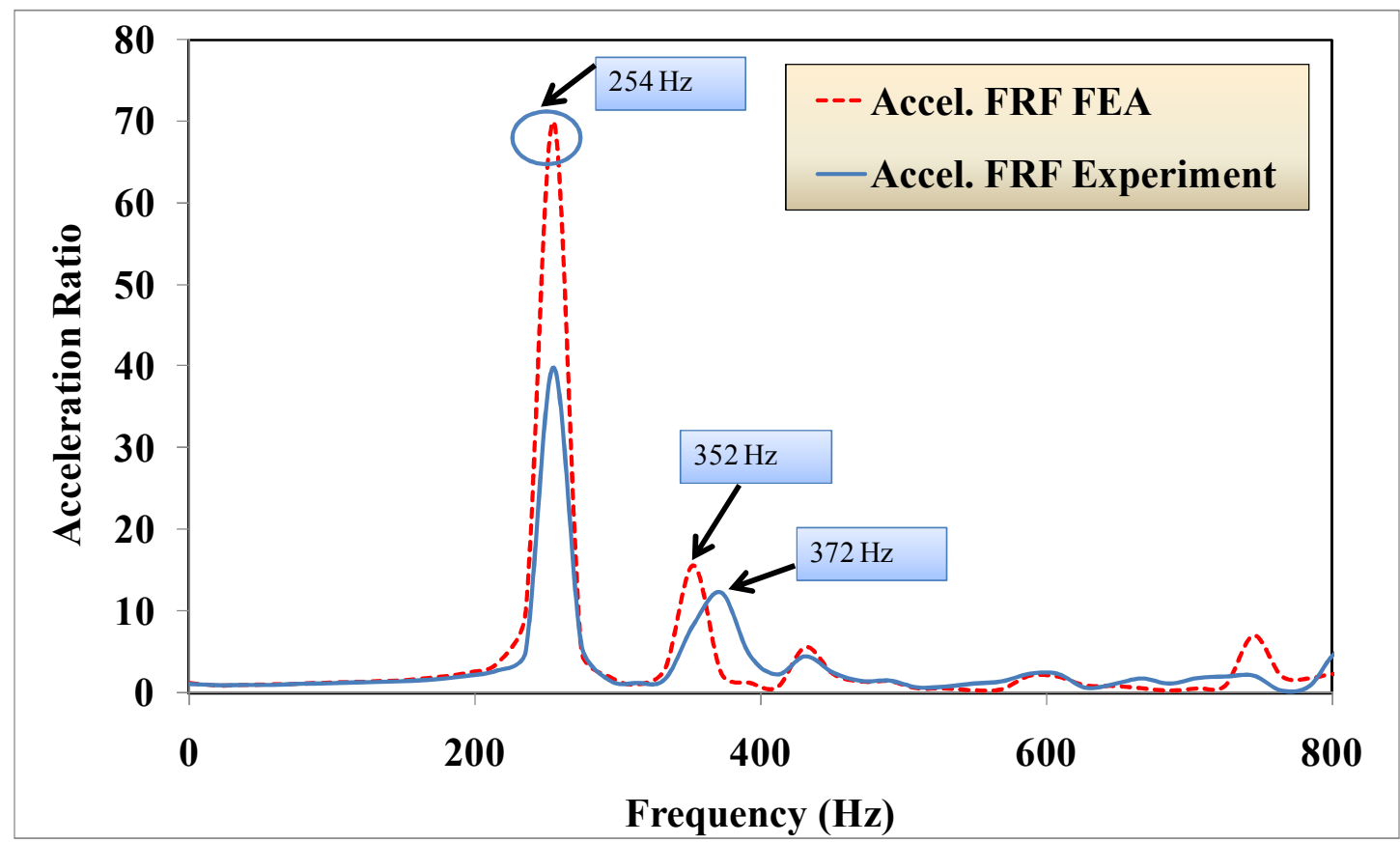

Figure 3-8 Frequency response function of the PWB measured at the accelerometer location B in Figure 2-7.

\subsubsection{Finite Element Model of Spring-Mounted PWB}

The FEA model of the spring-loaded PWB is very similar to that of the clamped PWB, as described in the previous section. In addition to the boundary conditions applied to the clamped PWB, the Au-plated leaf springs are rigidly constrained to the housing. To model the behavior of the Au-plated leaf springs, two translational Cartesian connection components are defined. Cartesian spring connectors in ABAQUS provide a connection between two nodes that allows independent behavior in three local Cartesian directions. The spring constant in the out-of-plane direction is defined through these connectors. In the course of a separate study, these spring constants were found both through simulation and experiment to be $2.91 \mathrm{~N} / \mathrm{mm}$. These spring constants were found for a static case and consequently their values would not be exactly the same for dynamic loading. Therefore, our aim is to find out how accurately the combined model (PWB \& leaf springs) can predict the 
experimental response to dynamic loading. These spring constants are expected to be more effective at predicting the lower natural frequencies with diminishing effectiveness for higher modes. The schematic of the spring-loaded model is shown in Figure 3-9.

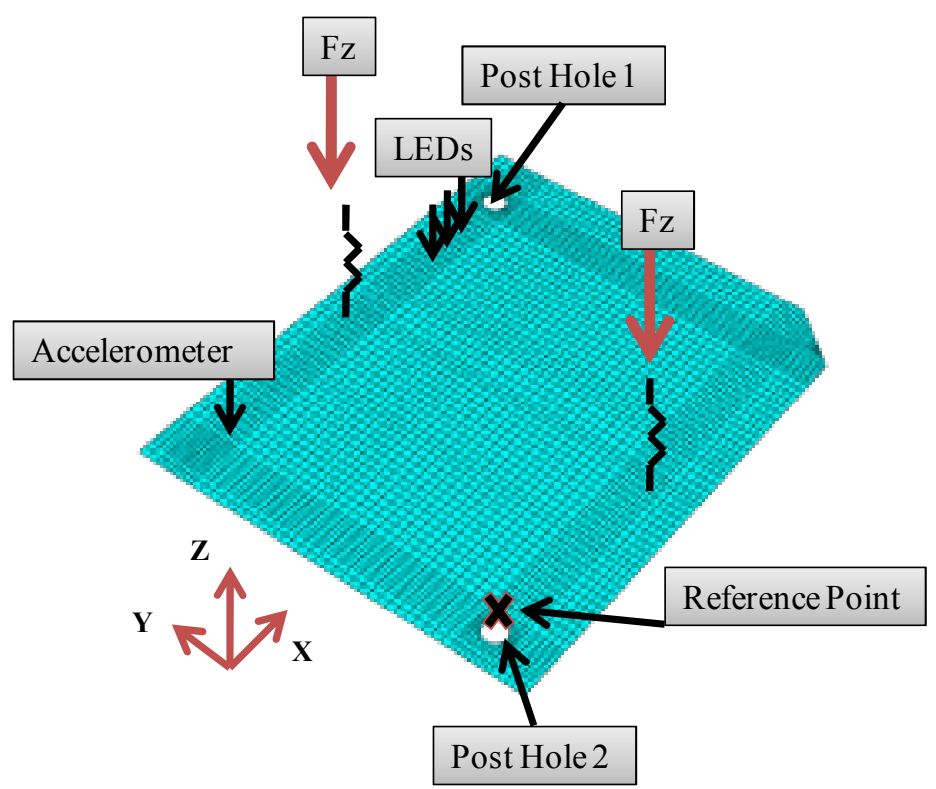

Figure 3-9- Spring-Mounted model

As in the prior study on the clamped PWB, the natural frequencies are first determined through modal analysis using ABAQUS/Standard ${ }^{\mathrm{TM}}$. The material properties and Raleigh damping factors are the same as those used earlier in Section 3.3.1 to model the clamped PWB. Figure 3-10 compares the FEA results for with the experimental results, for the spring-loaded case. As expected, the accuracy of this model is slightly less than that of the clamped PWB. Even though this model is less accurate it is still capable of generating the first two modes with good precision. From the graph below the first and second modes occur at $251 \mathrm{~Hz}$ and $366 \mathrm{~Hz}$, respectively. These values are underestimated by only $4.5 \%$ for the first mode and by $6 \%$ for the 
second mode by the model. As discussed previously, we suspect that the use of spring constants obtained from a static test has contributed to these slight disagreements between model and experiment. Additionally, the $\alpha$ value for the FEA model also changes as the boundary conditions change in the spring-loaded case. Results show that $\alpha$ remained relatively unaltered for the first mode but had to be increased for the second mode by a factor of 1.8 to $35 \mathrm{Rad} / \mathrm{s}$ to obtain a good match between the predicted and measured acceleration amplitudes.

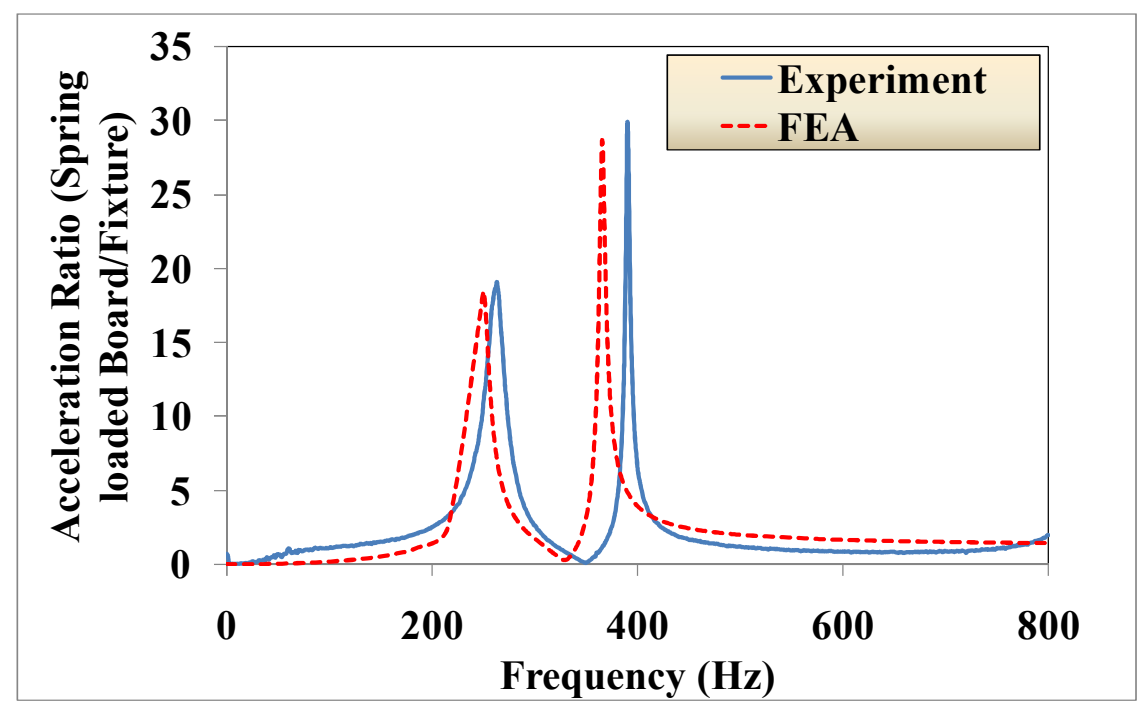

Figure 3-10- Natural Frequencies of the Spring Loaded Board

\subsubsection{Modal Analysis of Empty Housing}

To facilitate the finite element model development, the curved edges and corners of the housing are approximated with straight edges. Additionally, features that do not affect the dynamic response of the system are eliminated from the model, as shown in Figure 3-11 and discussed below. Using these simplifications significantly simplifies the meshing process and reduces the number of the elements 
used in the model, thus resulting in reduction of simulation time. These modifications are explained in detail in this section.

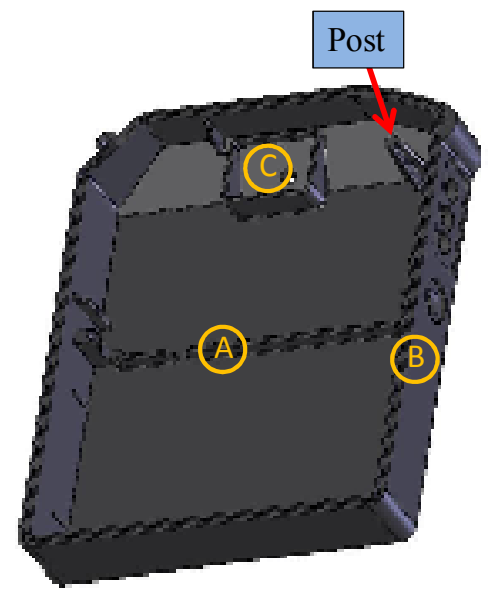

(I)

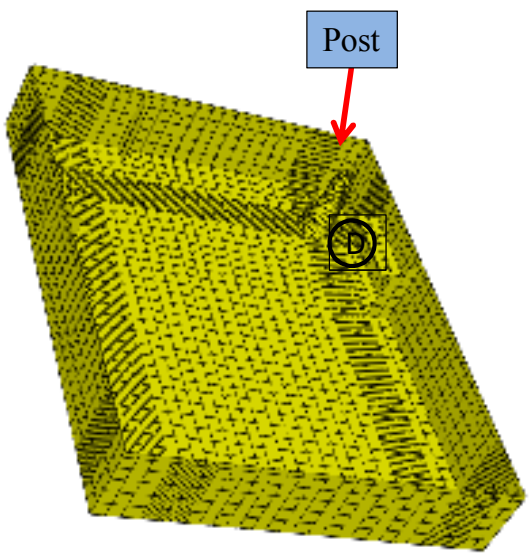

(II)

Figure 3-11- Actual device design versus simplified FEA model. (I) Bottom housing of actual device (II) Bottom housing of FEA model

The stopper in Figure 3-11, which is marked by letter "A", keeps the battery case aligned during the ultrasonic welding process and has no other function. Therefore, it is not included in the FEA model. As discussed in the prior sections, the LEDs and microphone do not physically exist in the model but instead their masses are added as distributed masses to their designated areas on the PWB. Therefore, the three LED holes on the edge B as well as the microphone ribs marked by letter "C" are also not included in the FEA model. Moreover, as discussed previously, the Auplated leaf springs are replaced by spring connecters in the FEA model. As a result, the charge contact pins do not affect the dynamic response of the system without the existence of Au-plated leaf springs anymore and for that reason the hole in which the contact charge pin is inserted on edge B is eliminated. The extruded part " $\mathrm{D}$ " is added to the FEA model to simulate any potential impact between the LEDs and the bottom 
case, as a result of drop impact. The location of the extruded part with respect to the PWB in the model assembly is determined by measuring the clearance between the LEDs and the bottom case in the actual product. This extruded part is modeled as a rigid extension so it will not deflect during simulation. Additionally, the star shaped post of the bottom case is modeled as a tapered circular post.

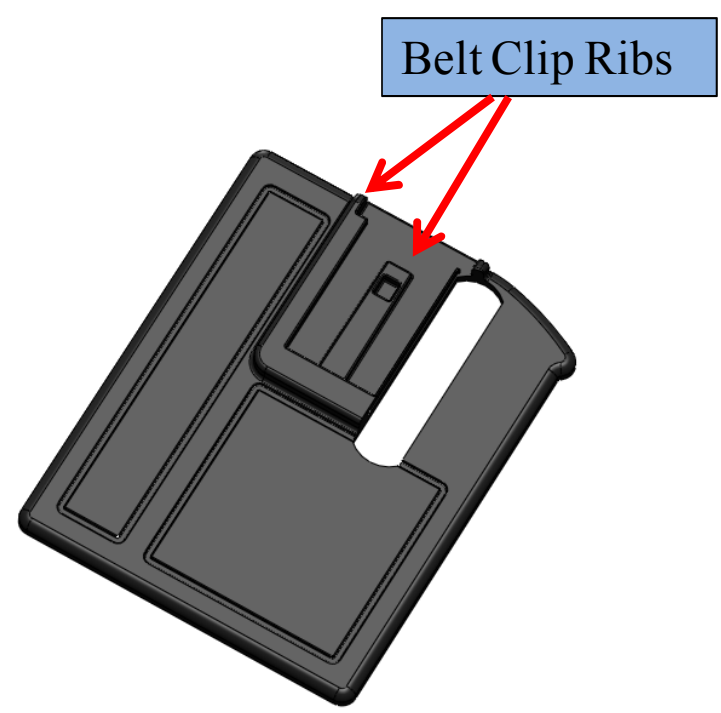

(I)

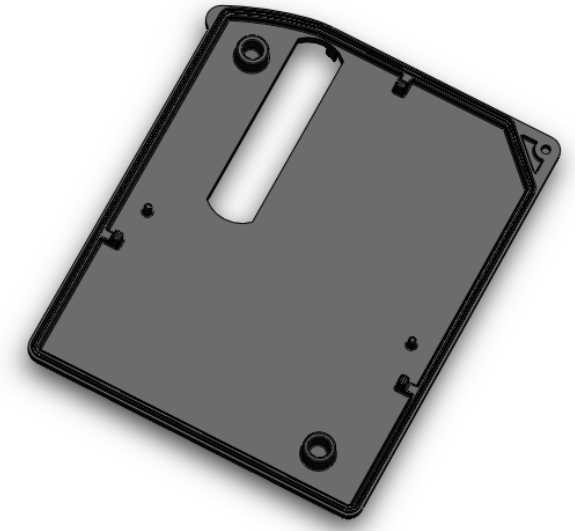

(III)

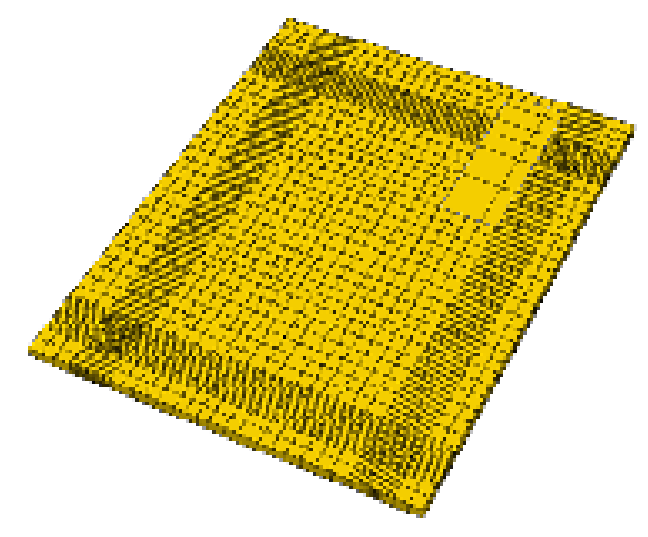

(II)

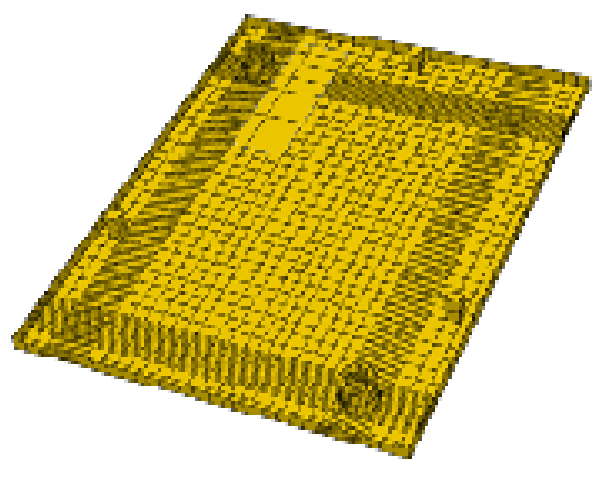

(IV)

Figure 3-12- Actual device design versus simplified FEA model- Top Housing. (I) and (III) Top housing of actual device (II) and (IV) Top housing of FEA Model

Figure 3-12 illustrates the geometrical differences between the actual device top case and its equivalent FEA model representation. As shown in Figure 3-12, the 
belt clip ribs are not included in the FEA model. However, since the belt clip ribs locally stiffen the top case, the modulus of elasticity of the housing is proportionately increased locally in the finite element model in under the footprint of the belt clip ribs (Refer to Figure 3-13). To calculate the equivalent modulus of elasticity for the stiffened areas, the rigidity (EI) of the L-shaped cross-section of the belt ribs are set equal to that of the stiffened areas, as shown in Figure 3-13, and Equation 3.3 is solved for the equivalent modulus $\mathrm{E}_{2}$ :

$E_{1} \times I_{1}=E_{2} \times I_{2}$

Equation 3.3

Where $\mathrm{E}$ is modulus of elasticity and $\mathrm{I}$ is the second moment of inertia. Subscript 1 indicates the physical configuration and subscript 2 indicates the equivalent simplified representation in the FEA model.

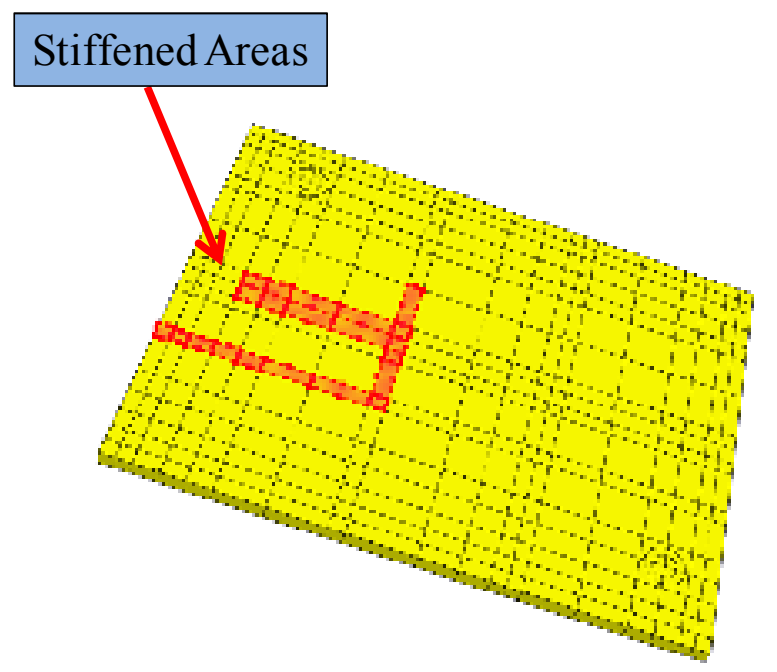

Figure 3-13- Stiffened areas to represent the stiffener ribs of the belt clip on the top housing 


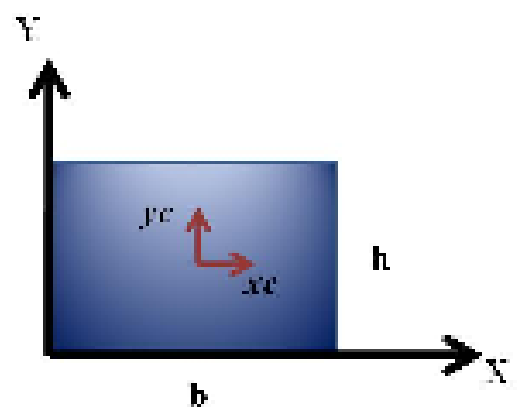

$I_{x c}=\frac{b h^{3}}{12}$

Equation 3.4

$I_{X}=I_{x c}+A d^{2}$

Equation 3.5

The second moment of inertia $I_{1}$ of the belt clip ribs is estimated based on the cross sectional area of the stiffener rib of the belt clip, shown in Figure 3-14. Likewise, Equation 3.5 is utilized to evaluate the equivalent modulus of elasticity for other portions of the belt clip ribs, shown in Figure 3-15 and Figure 3-16.

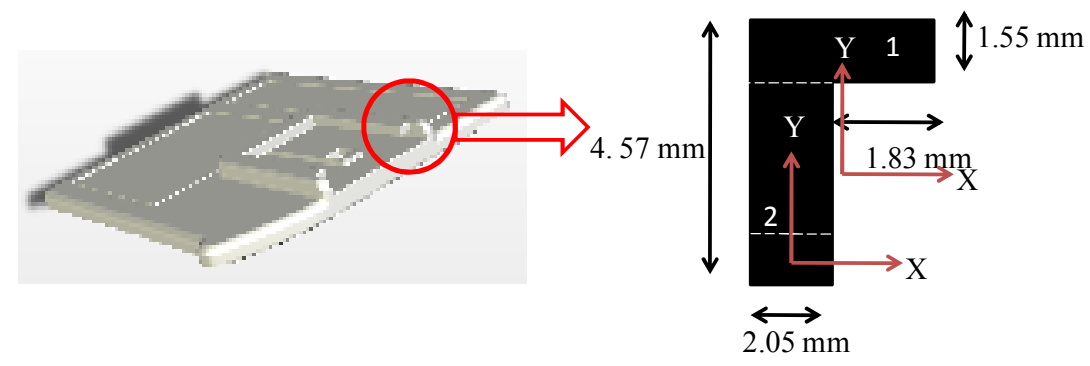

Figure 3-14- Cross sectional area stiffener ribs of the belt clip

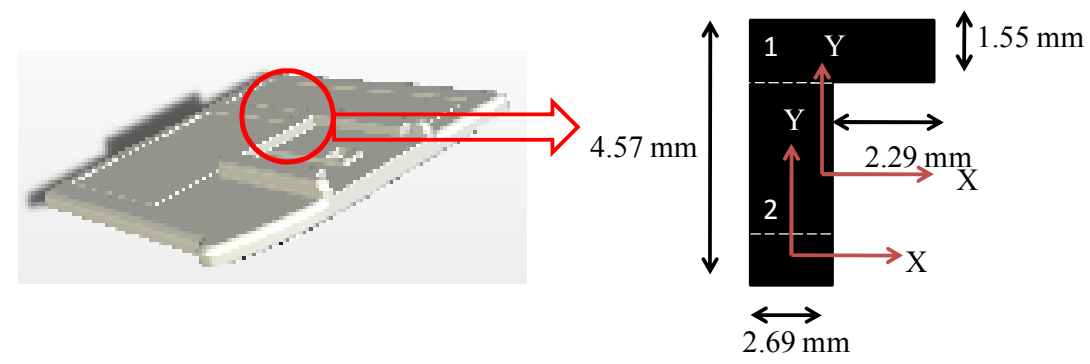

Figure 3-15- Cross sectional area stiffener ribs of the belt clip 


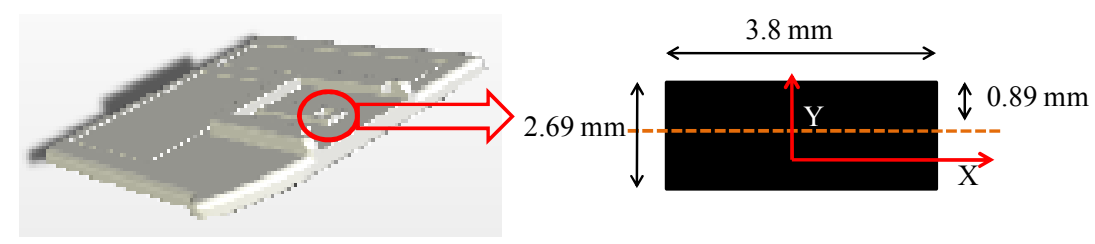

Figure 3-16- Cross sectional area stiffener ribs of the belt clip

Although these ribs stiffen the top housing predominantly in one direction, along the length of the rib, in the FEA model, isotropic $E_{2}$ material stiffness is assigned to these sections as well as to other parts of the housing. This is acceptable because the high geometric aspect ratio of the stiffener ribs provide structural stiffening only in one direction, even for isotropic material properties. Figure 3-17 summarizes the relationship between the housing modulus of elasticity and the stiffened areas.

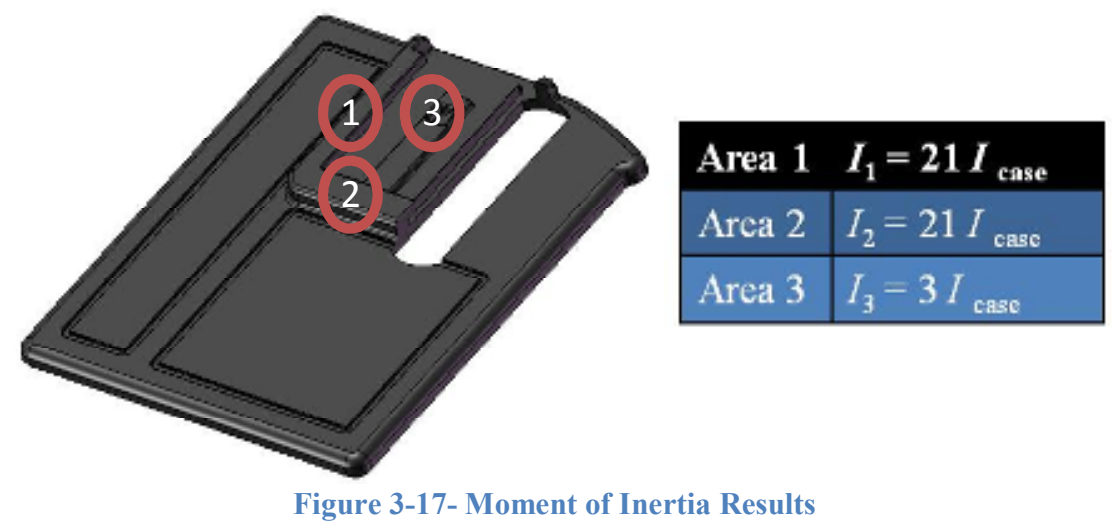

Furthermore, some of the material is removed from the top housing to accommodate the accelerometer on the PWB, as shown in Figure 24. This area in the housing is removed in the FEA model. The unmeshed areas in ABAQUS ${ }^{\mathrm{TM}}$ are not included in the FEA simulations. Figure 3-18 demonstrates the differences between the actual battery cover and the one in the FEA model. As shown, the geometries of 
the two are similar, except for the star shaped cap on the top of the battery cover, which is modeled with a circular cylinder.

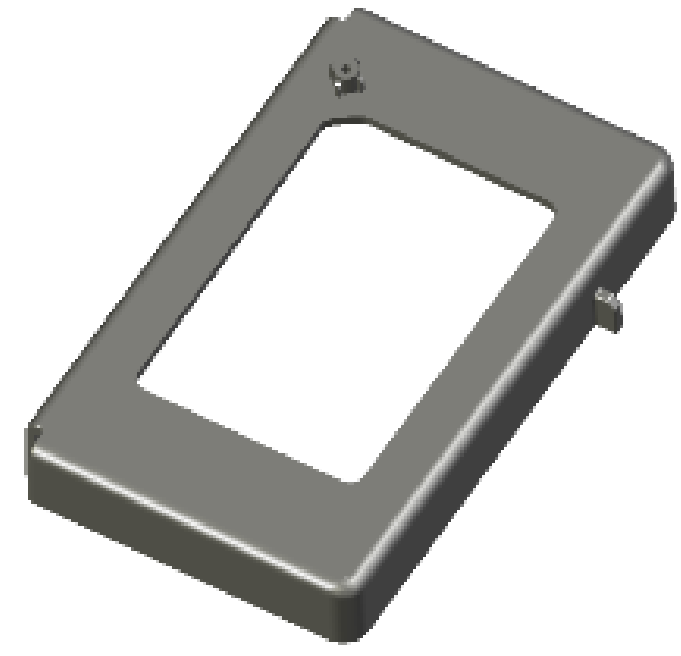

(I)

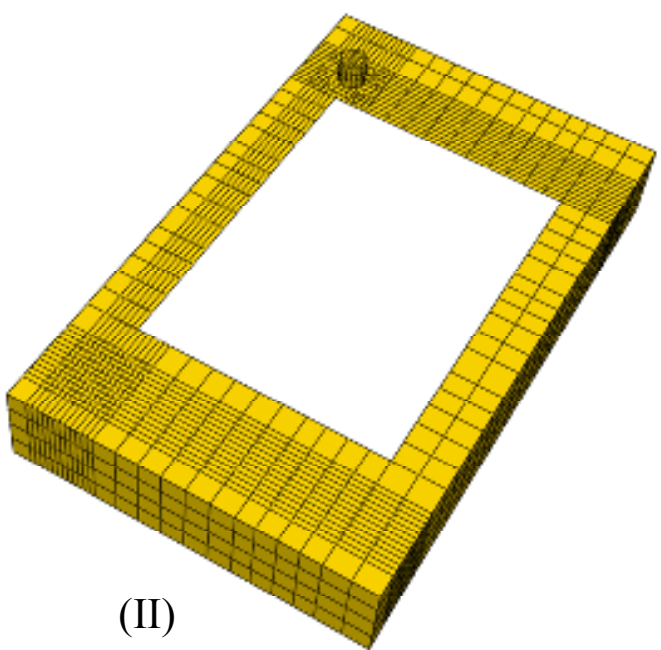

(II)

Figure 3-18 Battery Case Geometry vs. Model

The battery cover is tied to the bottom housing along its edges. Similarly, top and bottom housing are tied together (Refer to Figure 3-19). The bottom of the housing is modeled as a rigid section, since it is clamped to the fixture table. For modal analysis, the reference point assigned to the rigid body motion is fixed in all directions. Accelerometer masses are added to the system as distributed masses at their designated locations. 


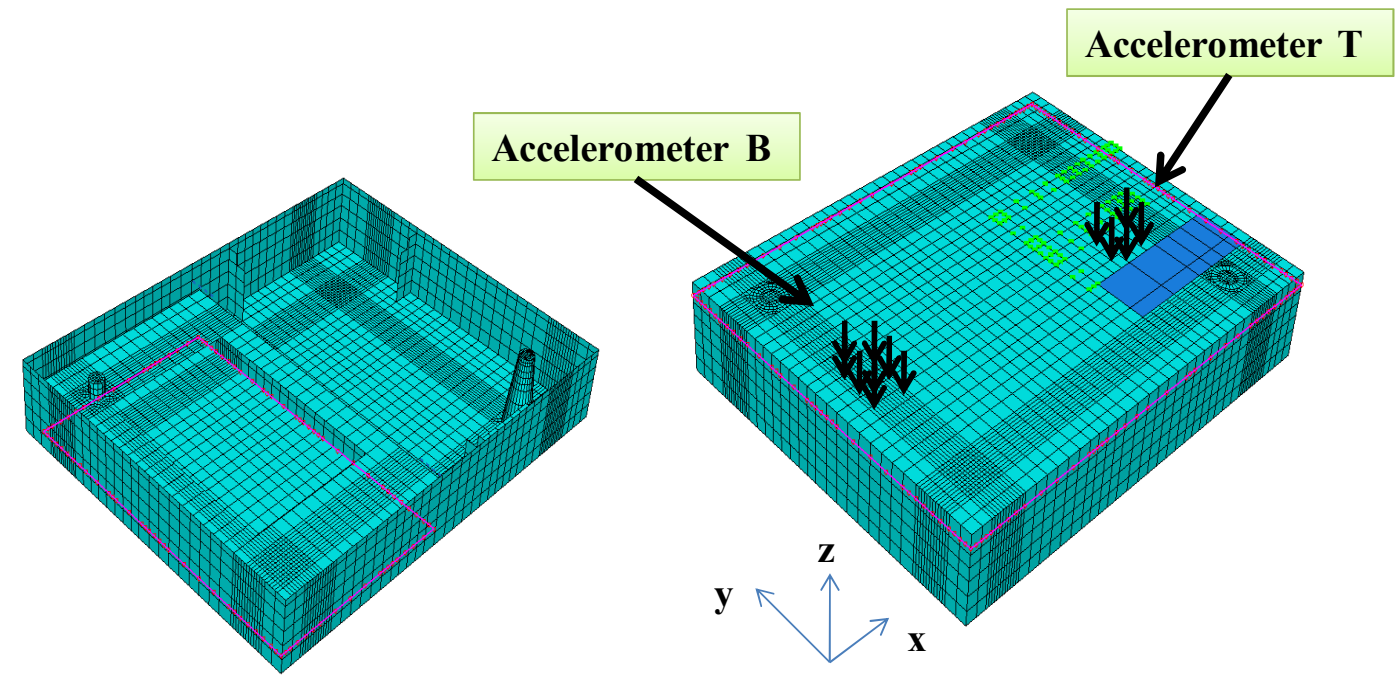

Figure 3-19- FEA model of empty case assembly- Accelerometer masses are added to the model as distributed masses as shown in the figure. Acceleration is measured in the $\mathrm{z}$ direction.

Four-node quadrilateral, stress/displacement shell elements with large-strain formulation are used to model top and bottom housing as well as the battery case. On the other hand, 8-node linear brick elements are used to model posts and caps. Similar to the bare board modal analysis, a frequency step followed by a mode-based steady state dynamic step is configured to find the natural frequencies of the empty case. In the mode-based steady state dynamic step the empty case is subjected to a $1 \mathrm{G}$ harmonic base excitation. Subsequently, the damping coefficient $\alpha$ for each mode was obtained by matching the acceleration amplitudes of simulation and experimental results. The mode shapes as well the frequency response function of the empty case are illustrated in Figure 3-20 and Figure 3-21. As shown, the first two natural frequencies of the FEA model match the experimental results to a good accuracy. The $\alpha$ values for the first two modes are 225 and $255 \mathrm{Rad} / \mathrm{s}$, respectively. These values 
differ from the $\alpha$ value obtained from the cantilever beam modal analysis $(\alpha=110)$, clearly indicating that Raleigh damping coefficients are structure-dependent and not strictly material properties.

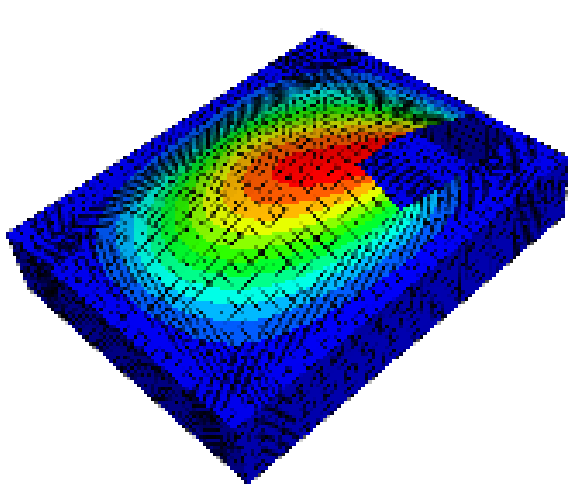

MODE 1

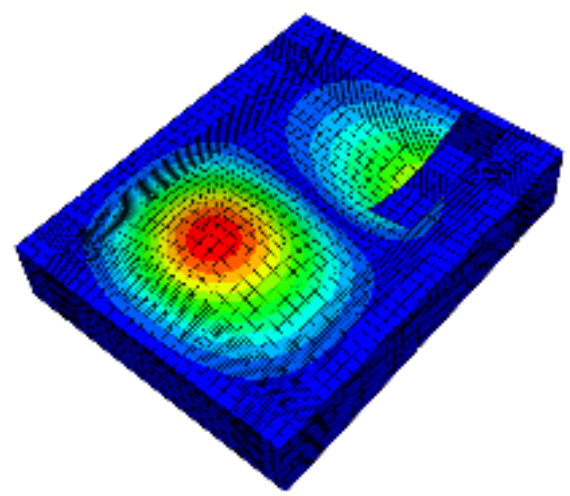

MODE 2

Figure 3-20 Dynamic mode shapes for the empty housing

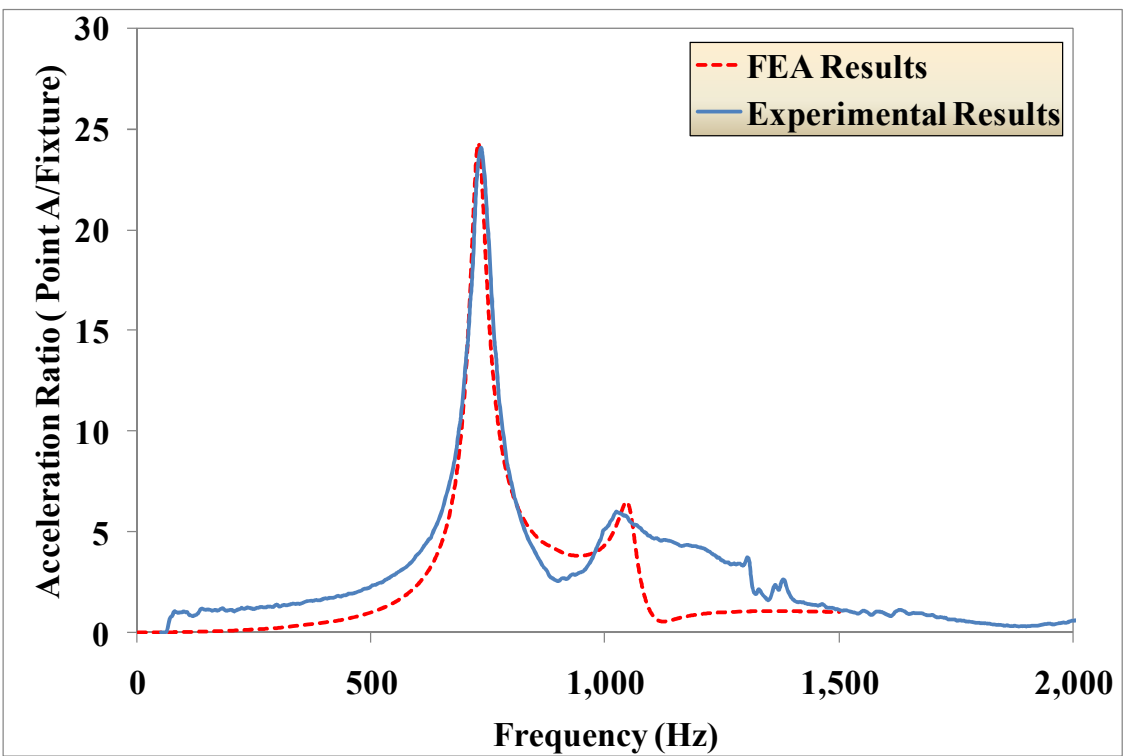

Figure 3-21- Frequency response function of the empty housing measured at the accelerometer $\mathrm{T}$ shown in Figure 2-12 


\subsubsection{Broad Band Simulation of Full Product Assembly}

In part I of the study an attempt was made to distinguish among the natural modes of the full product assembly that were driven by the PWB and the plastic housing. In section 2.5.1.4, it was concluded that the first two natural modes of the system were likely driven by the plastic housing; but no conclusion was drawn on the remaining natural modes. The last part of this section, tries to determine whether or not the remaining natural modes are the natural modes of the PWB. To find the natural frequencies of the PWB, modal analysis needs to be performed on the PWB with the same constraints that the PWB is subjected to in the full product. In the full device, the PWB is supported by the top housing stoppers, caps, and the bottom housing ribs. Therefore, as shown in Figure 3-22, the PWB is fixed at the support locations. Additionally, the masses of the accelerometer, LEDs, and the microphone housing are added as distributed masses at their designated locations (Refer to Figure $3-22)$.

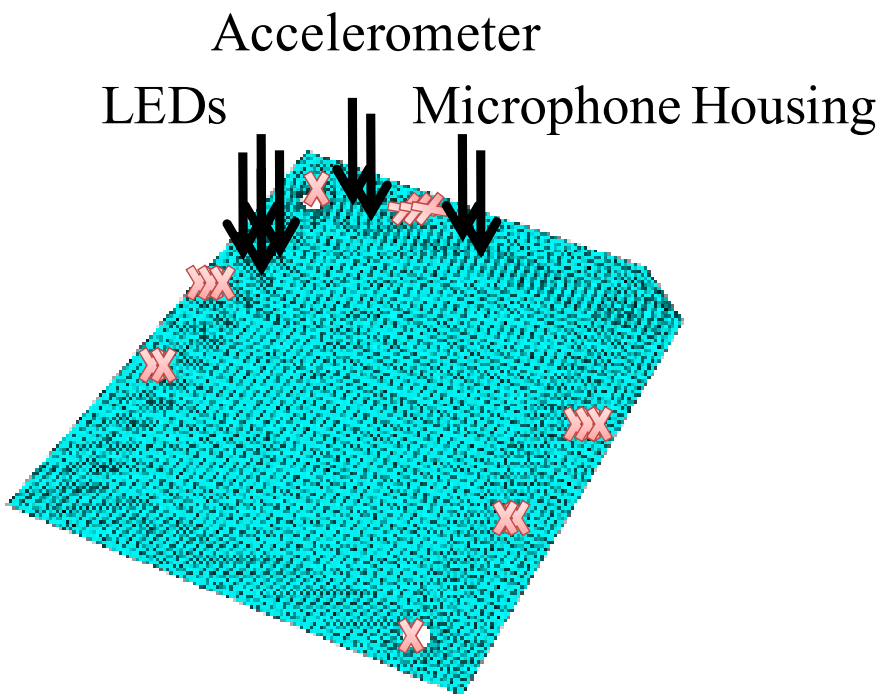

Figure 3-22 The red crosses indicate the areas at the which the PWB is fixed to roughly simulate the boundary conditions of the PWB inside the full product. 
Figure 3-23 illustrates the mode shapes and the corresponding natural frequencies of the PWB when subjected to the boundary conditions similar to that of experienced in the full product. It is important to note the modal analysis results shown in Figure 3-23 are only an estimate and do not represent the exact natural frequencies of the full product which are driven by the PWB. This is mainly due to the fact that this analysis lacks the interactions between the PWB and other components of the full product such as the battery cover. Additionally, the impact among the internal components during the test is a non-linear effect, which could change the natural frequencies of the system. However, it is expected that the values of the natural frequencies of this model to be close to that of the full product driven by the PWB. The first natural frequency of the PWB, $1154 \mathrm{~Hz}$, as shown in Figure 3-23 is very close to the second mode of the full product $(1152 \mathrm{~Hz})$, which was discussed in Section 2.5.1.5, Figure 2-33. In Section 2.5.1.5, it was also concluded that the second mode of the full product is likely driven by the plastic housing. Therefore, according to the modal analysis results of this section, it is possible that the natural frequencies of the PWB and empty housing are coinciding at this particular frequency. Furthermore, the third natural mode of the full product, which occurs at $1356 \mathrm{~Hz}$, is very close to the second natural frequency of the PWB obtained in this section $(1300 \mathrm{~Hz})$. As a result, it can be concluded that the third mode of the full product is driven by the PWB. The fourth natural frequency of the full product $(1400 \mathrm{~Hz})$ does not correspond to the third mode of the PWB shown in Figure 3-23. Therefore, no conclusion can be made with certainty on whether or not the forth natural mode of the full product was driven by the PWB or the plastic housing. 

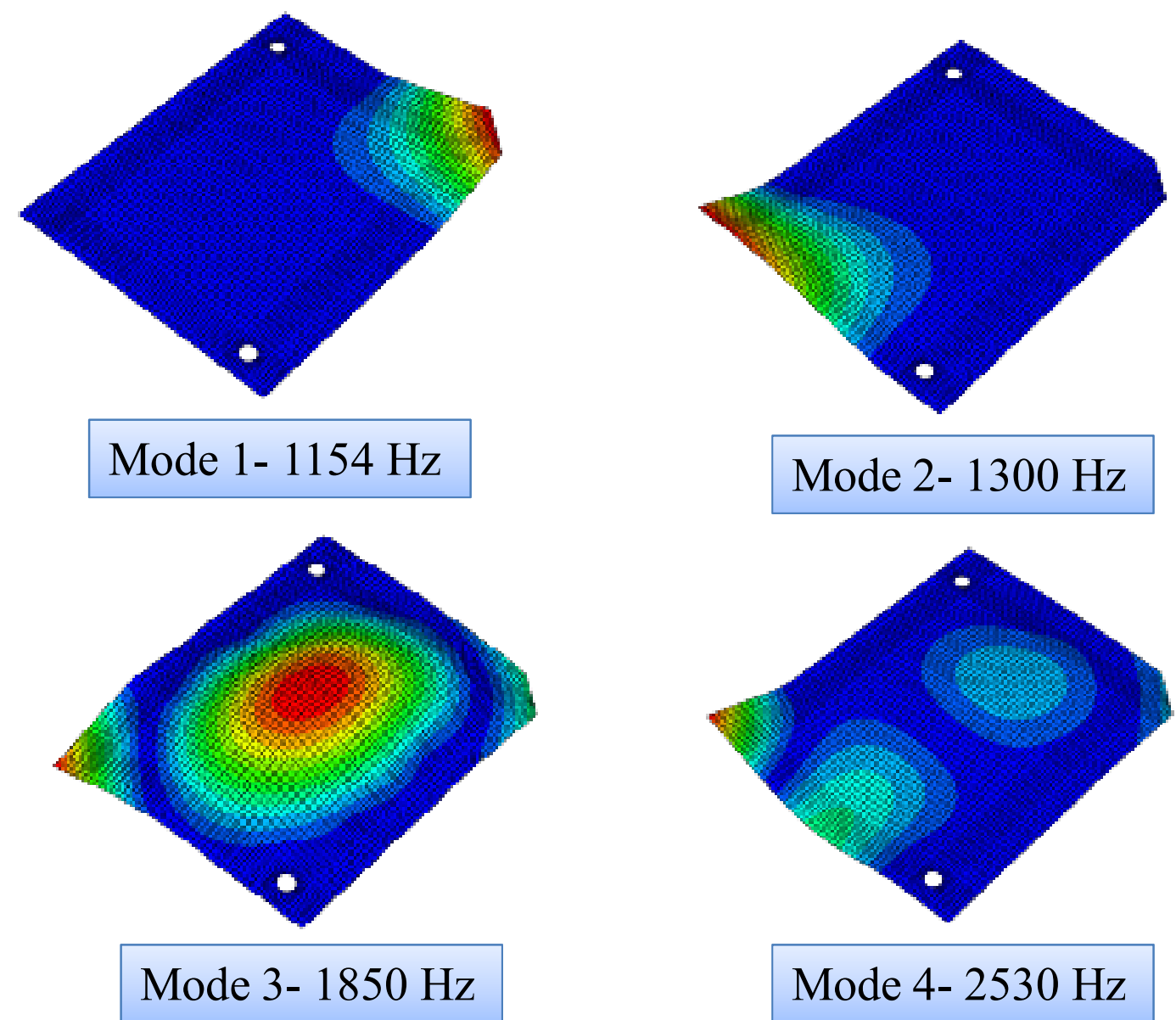

Figure 3-23- Mode shapes and natural frequencies of the PWB when subjected to the constraints similar to that of experienced in the actual device. 


\subsubsection{Shock Simulation of Full Product Assembly}

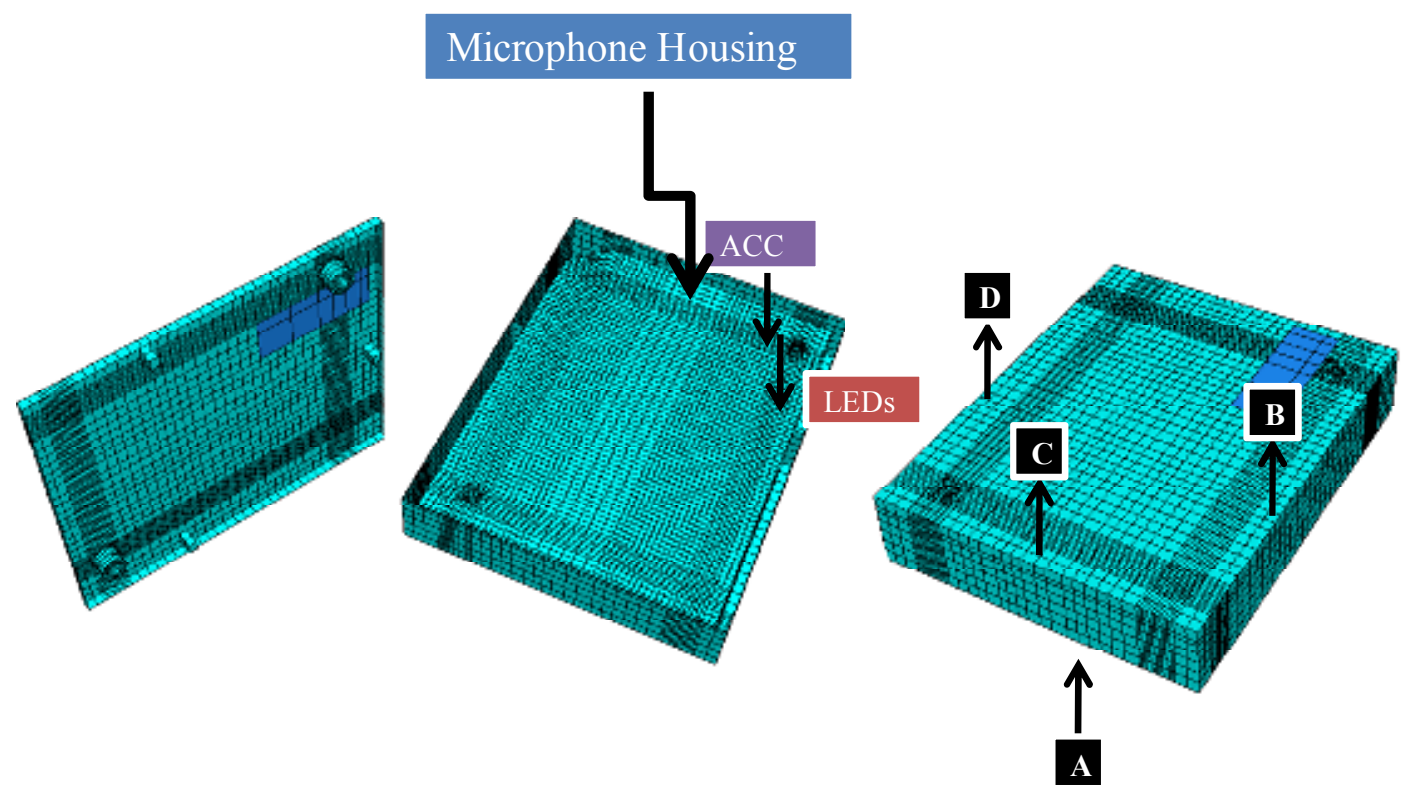

Figure 3-24- Full Product Assembly

To construct the FEA model of the full product, the PWB is inserted in the empty case assembly which has been discussed in the previous section. The same material properties and Raleigh damping factors that were obtained in the preceding sections for the PWB and the plastic housing are used in the full product FEA model. The bottom of the product as well as the areas that are fixed by the clamps (as shown in Figure 2-18 of Part I) are modeled with rigid constraints. The input shock pulse is applied as an acceleration boundary condition to the reference points that are assigned to the rigid areas. These areas are marked by letters, A, B, C, and D in Figure 3-24. Additionally, accelerometer and LED masses ( 0.9 gms and 0.9 gms, respectively) are added to the model as distributed loads at their designated areas, as shown in Figure 3-24. For simplicity, frictionless contact is defined between all components of the system, to model any potential impact among the components during the shock response. The battery is tightly packed in the battery cover with a cushion. Since the 
battery is tightly mounted to the clamped bottom of the housing, it its effect on the dynamic response of the PWB is ignored for the particular orientation under study.

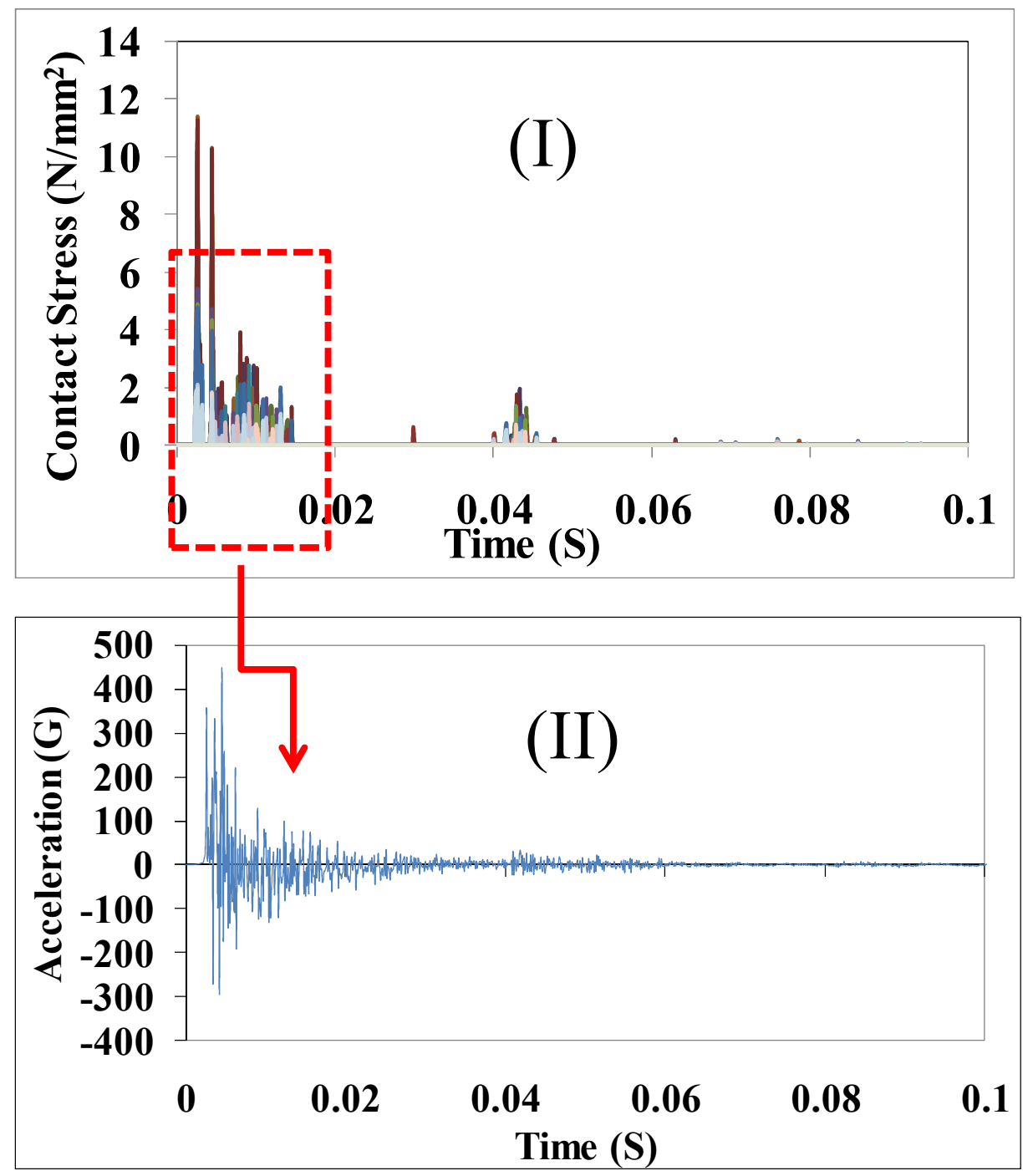

Figure 3-25- Full product FEA results when the board is not fixed to the top housing caps. (I) illustrates the contact stresses around the bottom housing post caused by impact between the guide post on the housing and the PWB guide hole. (II) Demonstrates the unrealistic accelerations predicted by the FEA model due to the impact of internal components.

As discussed previously in Section 2.5.1.5 of Part I of this study, the measured acceleration magnification factor for the full product shock test was roughly 1.15 . However, Figure 3-25 demonstrates that the acceleration magnification factor predicted by the FEA model is approximately 10. Figure 3-25 also illustrates the 
contact stresses around the bottom housing post caused by impact between the guide post on the housing and the PWB guide hole. These results suggest that the high acceleration levels in the FEA model are results of impact between the internal parts, e.g. between the PWB and the post. Similar impact can also occur between the PWB and other parts of the housing (e.g. the ribs on the bottom housing, the stoppers on the top housing), and also between the LED and the housing, and between the microphone and the housing. These impact-induced high accelerations are not seen in the actual product shock test, because the contact surfaces are neither frictionless, nor elastic. To truly capture the contact stresses and the impact decelerations correctly, the contacts must be modeled with suitable damping and non-conservative behavior. Additionally, the strain gage and accelerometer wires routed inside the device can also provide constraints that significantly lower the acceleration levels experienced by the PWB.

Furthermore, the fingers that clamp down the device to fixture could lower the clearance between the PWB and other components. Therefore, to more accurately simulate the real test boundary conditions, the PWB is fixed to the top housing caps to prevent some of contact stresses between the PWB and bottom housing post. Figure 3-26 compares the acceleration response of the PWB predicted by the FEA model with that of the experiment. As shown, the peak acceleration value, as well as pulse duration obtained from FEA modeling match the experimental results quite well. Additionally, both acceleration profiles follow the same trend. The accuracy of the FEA results indicates that unrealistically high elastic contact stresses were indeed 
the main source of the unrealistically high acceleration levels observed in the original FEA model.

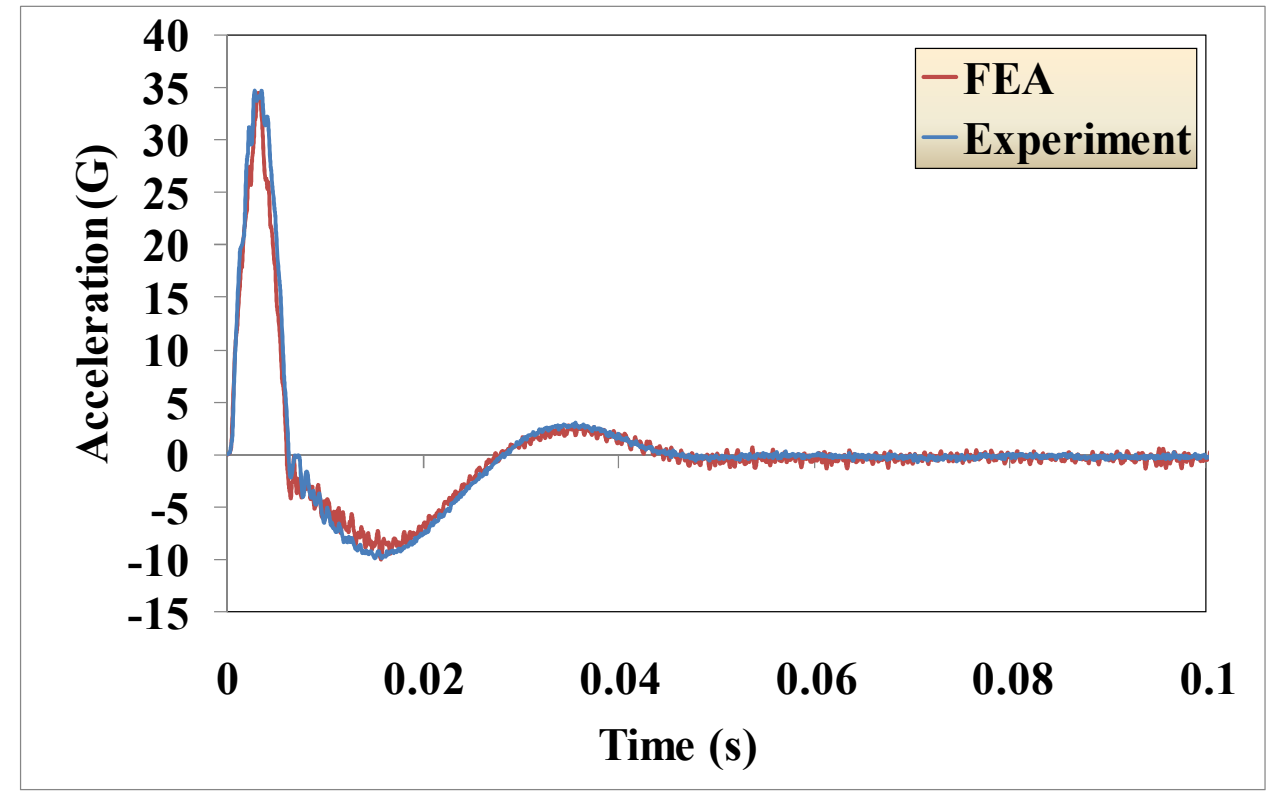

Figure 3-26- Shock test FEA results VS. Experiment results. The acceleration is measured on the PWB in the $z$ direction as shown in Figure 3-24.

\section{$\underline{3.5 \text { Drop Test Modeling Results }}$}

Section 3.5 discusses the drop test modeling of the clamped PWB and the full product assembly. Section 3.5.1 demonstrates the differences between modeling for shock and drop loading. Section 3.5.1 attempts to improve the same FEA model developed for the shock test of the full product, discussed in Section 3.4.5., by performing a parametric study on various parameters of the Contact Property module of ABAQUS/Explicit. 


\subsubsection{Drop Modeling of clamped PWB}

The drop finite element model for the clamped PWB is developed in a similar fashion to that of the shock model described in Section 3.3.1. The only difference is in the input excitation which is roughly $1600 \mathrm{Gs}$ and the damping parameters. For details of the model, refer to section 3.3.1. As described in Section 2.5.2.1, both natural modes of the PWB are excited in drop loading, because the significantly shorter pulse duration in the drop test does not produce any notches in the excitation spectrum at either natural modes of the clamped PWB. Therefore, unlike the shock modeling, the value of $\alpha$ is set to $70 \mathrm{Rad} / \mathrm{s}$ which corresponds to the first natural mode of the PWB. The readings of the accelerometer placed on the free end of the PWB, shown in Figure 2-40, are plotted against the FEA simulation results in Figure 3-27. The first peak of the acceleration is slightly higher than the corresponding experimental value shown in this graph. However, as explained in section 2.5.2.1, unlike strain measurements, there is a small variation in board acceleration response from one trial to the other. The details are discussed in section 2.5.2.1. This variation needs to be taken into account when comparing simulation results with experimental data. The mean value of the distribution which represents the experimental data is 2252 Gs. If the mean acceleration value of $2252 \mathrm{Gs}$ is compared with that of simulation, $2200 \mathrm{Gs}$, the error percentage will be roughly $2 \%$. On the other hand, the subsequent excitation peaks of the FEA graph are much larger than the experimental ones. This indicates that the $\alpha$ value estimated from a linear test (broad band test of the clamped PWB) cannot predict the response of the same PWB subjected to a different loading. Therefore, it can be concluded that $\alpha$ varies with loading possibly 
due to material non-linearities. Similarly, the first peak of the strain history is predicted pretty well compared to that of the experiment. However, the subsequent peaks are much larger as shown in Figure 3-28.

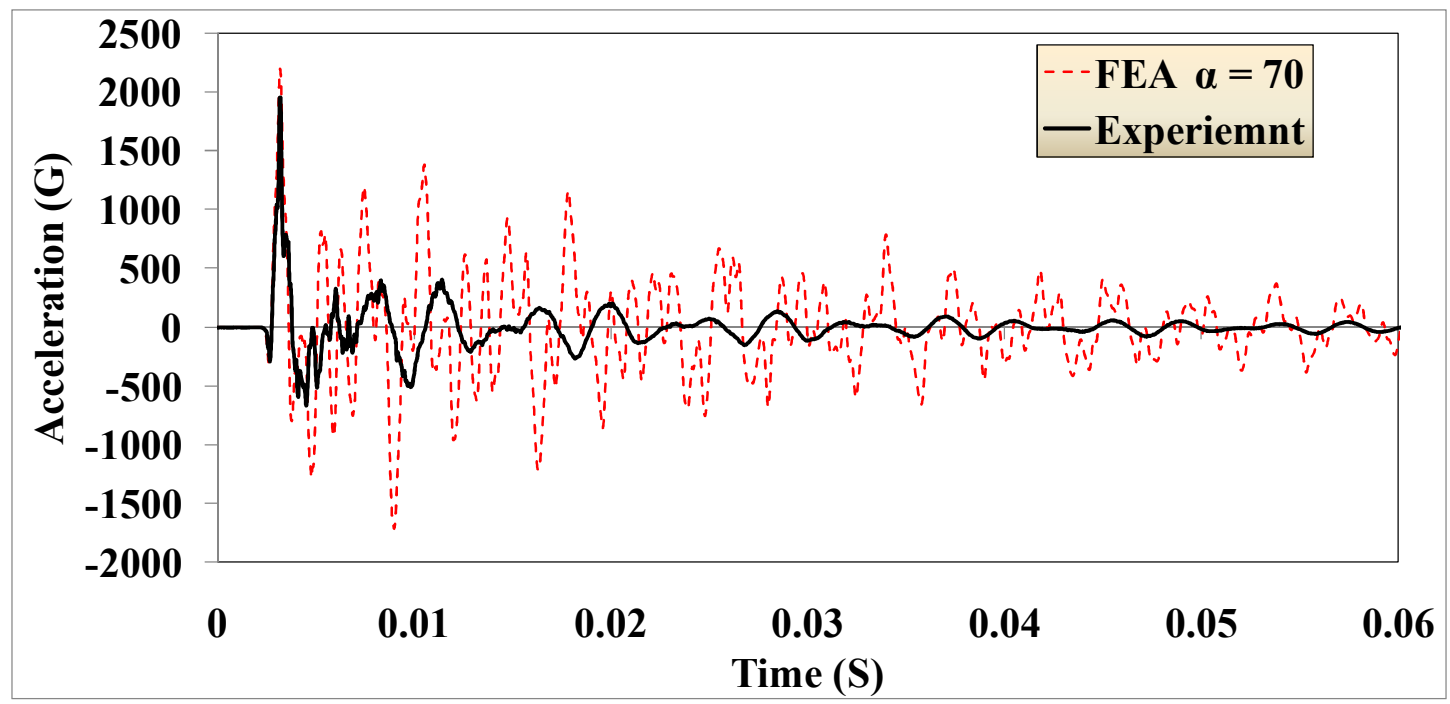

Figure 3-27- Acceleration at the free end of the clamped board as shown in Figure 2-39- FEA VS. Experiment

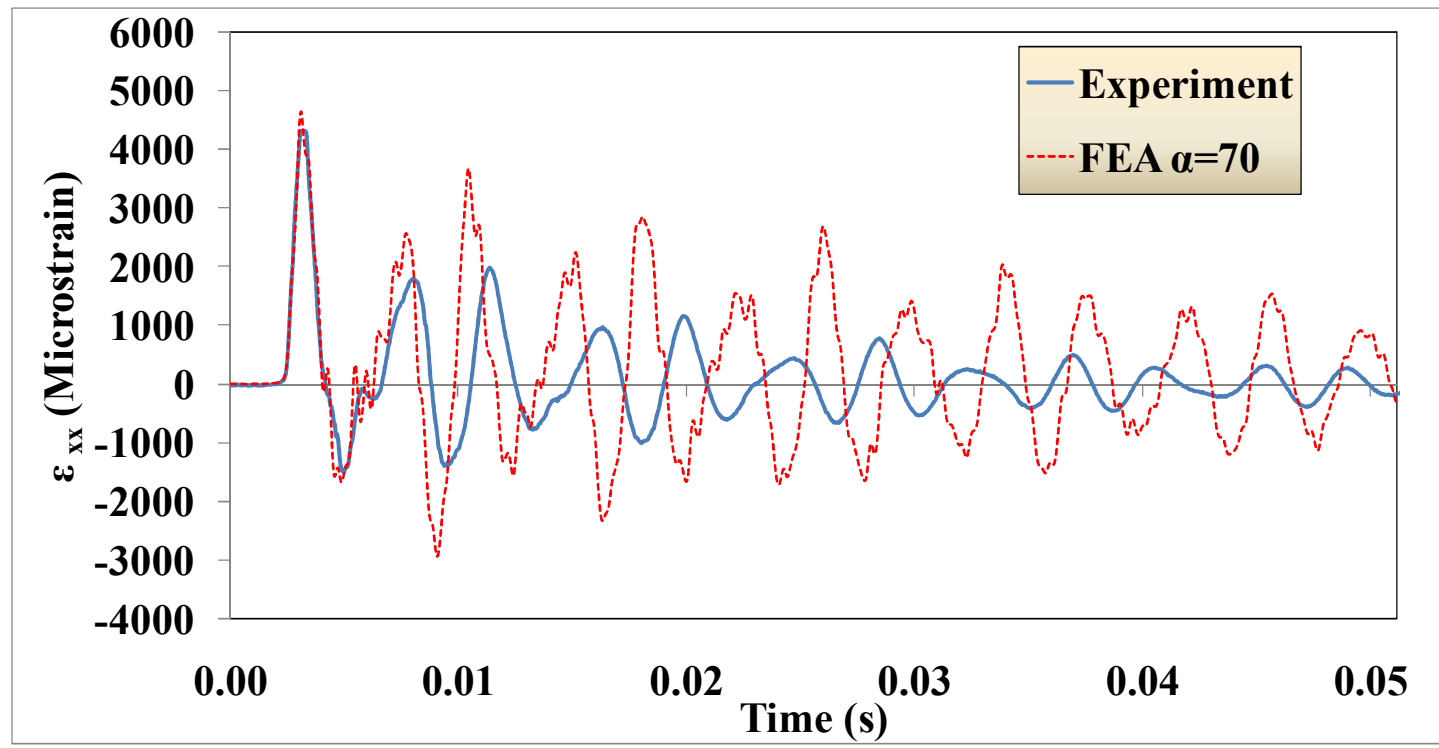

Figure 3-28 Strain at the post of the clamped PWB as shown in Figure 2-40-FEA vs. Experiment

To obtain a better agreement between FEA and experiment, the value of $\alpha$ is increased a by a factor of 2.5. As shown in Figure 3-29 and Figure 3-30, there is a very good agreement between the test and simulation results. However, if the mean 
acceleration value of 2252 Gs is compared with the simulation peak acceleration, 1993 Gs, the error percentage will be roughly 10\%. Additionally, the FFT response of the strain gage mounted next to the post of the PWB, shown in Figure 2-40, is compared to that of the simulation results in Figure 3-31. Figure 3-31 indicates that experimental and simulation results are in agreement with each other in the frequency domain as well. The slight discrepancy among the natural frequencies is within the measurement resolution $( \pm 40 \mathrm{~Hz})$. Similarly, the frequency function of the accelerometer at the free end of the PWB is shown in Figure 3-32.

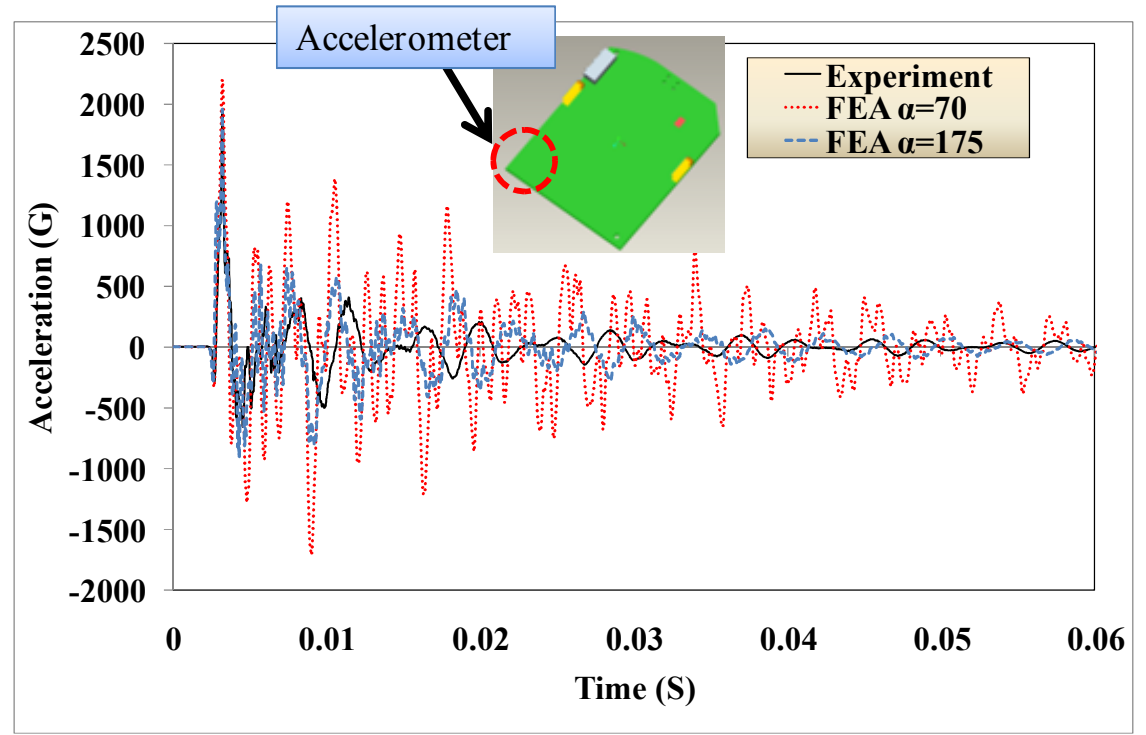

Figure 3-29- Acceleration at the Free End of the Bare Board - FEA VS. Experiment. $\alpha=175$ Rad/s for the case shown. 


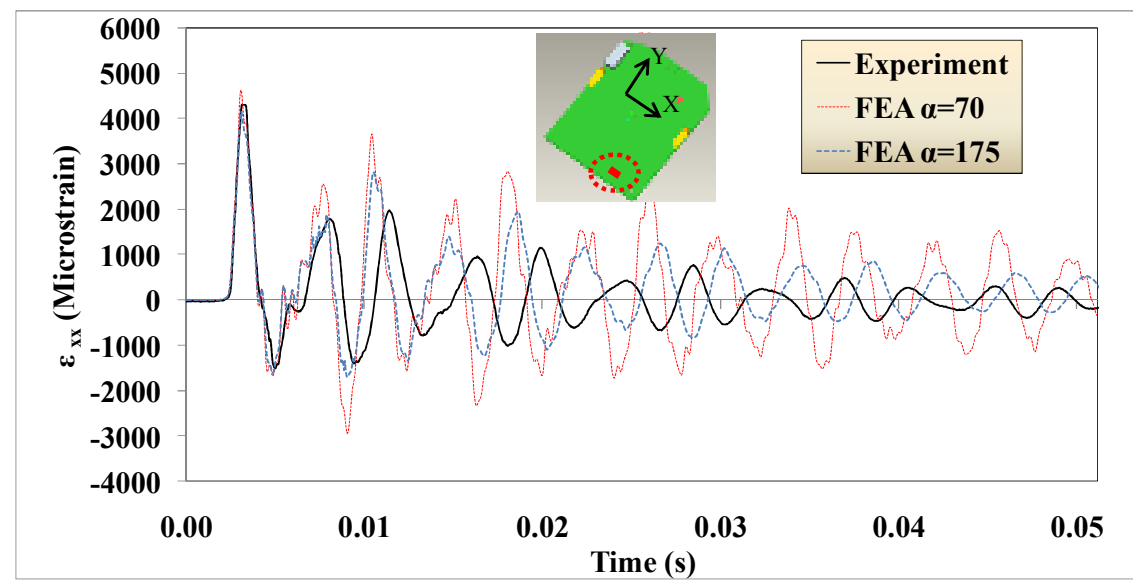

Figure 3-30- Strain at the post of the clamped PWB- FEA VS. Experiment. $\alpha=175 \mathrm{Rad} / \mathrm{s}$ for the case shown.

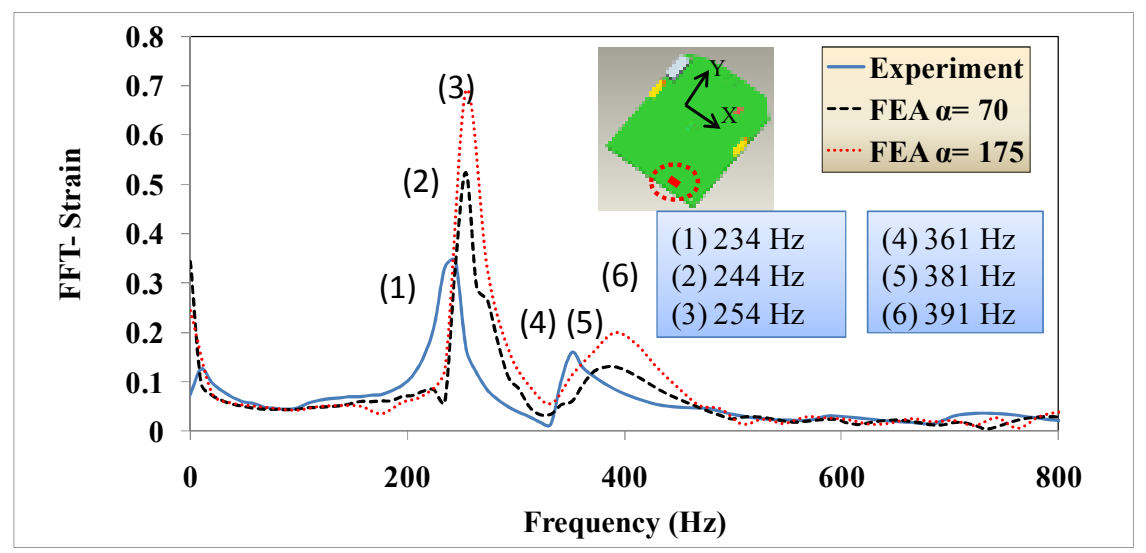

Figure 3-31- Strain FFT Response- FEA VS Experiment

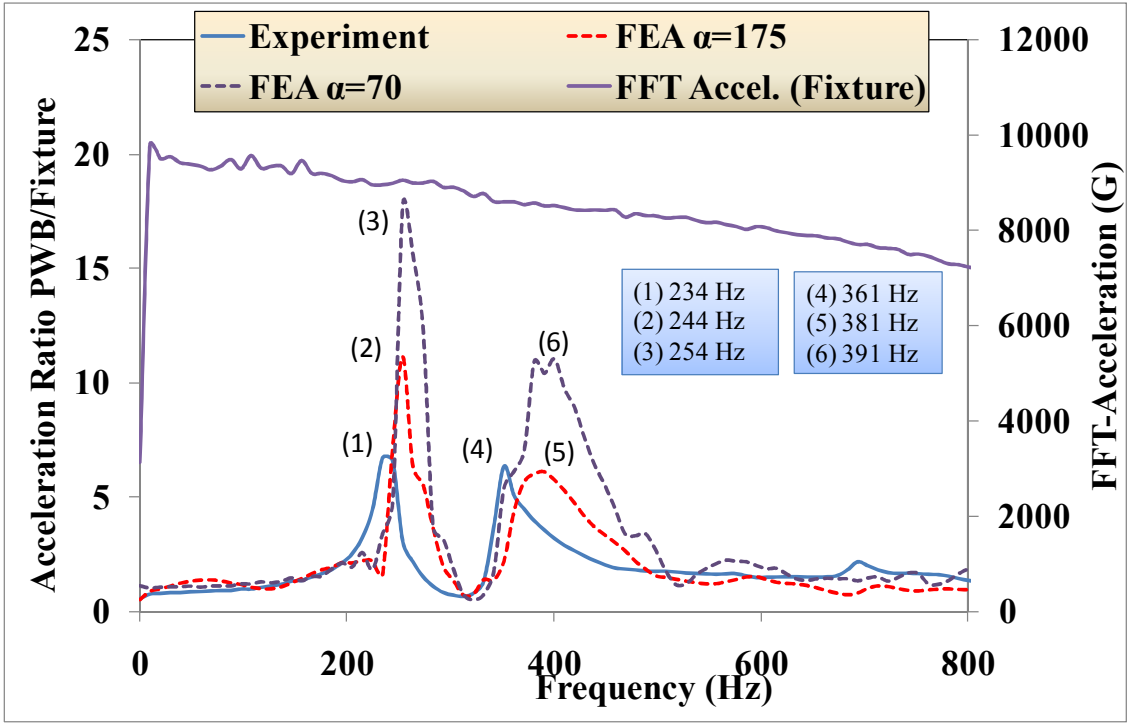

Figure 3-32- Frequency response function of the PWB measured at the accelerometer location shown in Figure 2-7. 


\subsubsection{Drop Simulation of Full Product Assembly}

The same model that was constructed for the shock simulation of the full product assembly is utilized to perform drop simulation on the full product. There are, however, some differences in boundary conditions and contact properties assigned to the model that will be discussed throughout this section. In the shock simulation of the full product, it was demonstrated that the unrealistic high accelerations resulted from the impact among the internal components can be prevented by fixing the top housing caps to the PWB. This technique, however, is not as effective in drop simulation. Despite fixing the PWB to the top housing caps, the internal components of the system still come in contact with each other. This leads to unrealistically high acceleration levels, because as discussed in section 3.4.5 the contact surfaces are neither frictionless, nor elastic. Therefore, suitable contact properties must be defined to capture the response of the full product.

When using linear elastic material properties in FEA models realistic estimates of the contact stresses and the impact decelerations require the use of suitable non-conservative features such as 'soft' contact, contact damping, and contact friction. In this study, a parametric sensitivity study was conducted to explore the various methodologies available in ABAQUS for modeling non-conservative contact. The goal was to find out how contact property parameters and damping parameters need to be defined to effectively simulate the effects of plastic deformation and contact friction and other dissipation mechanisms, using models with elastic material properties. 
The accuracy of the drop modeling assumptions are assessed by comparing two important parameters between the model and experiments: the cyclic range of the first response cycle after the drop impact, as well as the RMS value over the entire time history of the record. Although these metrics are monitored for both the acceleration response as well as the strain response at key locations on the PWB inside the plastic housing, priority is given to the errors in the strain prediction. This is due to the fact that flexural strain is one of the leading drivers for most failures on PWAs under drop loading. Additionally, matching the cyclic range of the first strain cycle also has priority over matching the RMS value, because roughly $90 \%$ of the drop damage occurs during the first cycle.

Three different types of mechanical contact property can be defined in ABAQUS/Explicit, which are as follows; Tangential and normal behaviors, as well as damping. In this paper, no parametric study is conducted on the coefficient of friction and a constant value of 0.3 is used for all cases. There are four different options in the normal behavior section that can define a pressure-overclosure relationship between surfaces. The four options include hard contact, as well as three types of softened contact relationships. The pressure-overclosure relationship for softened contact can be prescribed by using a linear law, tabular piecewise-linear law, or an exponential law. This study, however, only focuses on the hard contact, as well as the linear law to define a pressure-overclosure relationship. The only parameter that needs be prescribed for the linear law contact property option is contact stiffness which is the slope of the pressure-overclosure curve. In addition to the contact pressure overclosure relationship, a damping factor can be defined to oppose the relative 
motion between the surfaces. For this option, a unitless damping coefficient in terms of the fraction of critical damping associated with the contact stiffness needs to be defined.

Initially, an investigation is conducted to fully understand how acceleration, contact stresses, and flexural strain are affected when soft contact is used rather than hard contact. Histograms of acceleration and strain, as well as the acceleration and strain response of the PWB in the time domain, are studied to better visual the changes in the PWB response. For the purpose of this comparison, soft contact stiffness is set equal to $0.5 \mathrm{~N} / \mathrm{mm}^{2}$. As shown in Figure 3-33, defining soft contact reduces contact stresses enormously in comparison with when hard contact is defined.

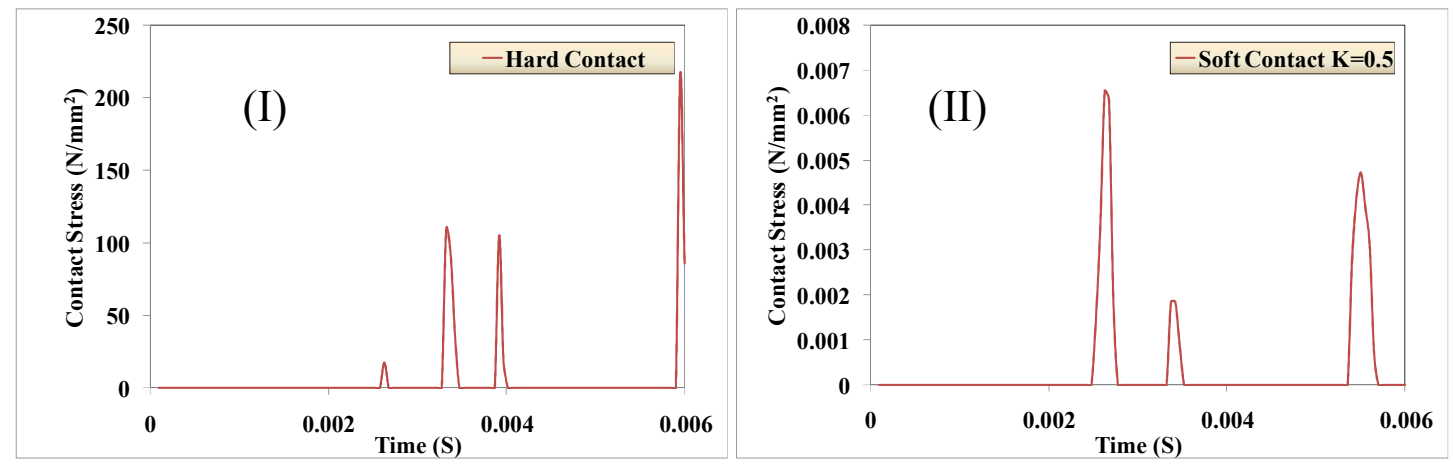

Figure 3-33 (I) Contact stresses at a node next to the tapered post when hard contact is defined. (II) Contact stresses at a node next to the tapered post when soft contact is defined.

Additionally, comparison of the experimental acceleration histogram, as well as the acceleration time response with the models that include soft and hard contact indicate that some of the high accelerations caused by the impact between the internal components of system disappear when soft contact is defined ( Refer to Figure 3-34 and Figure 3-35). 


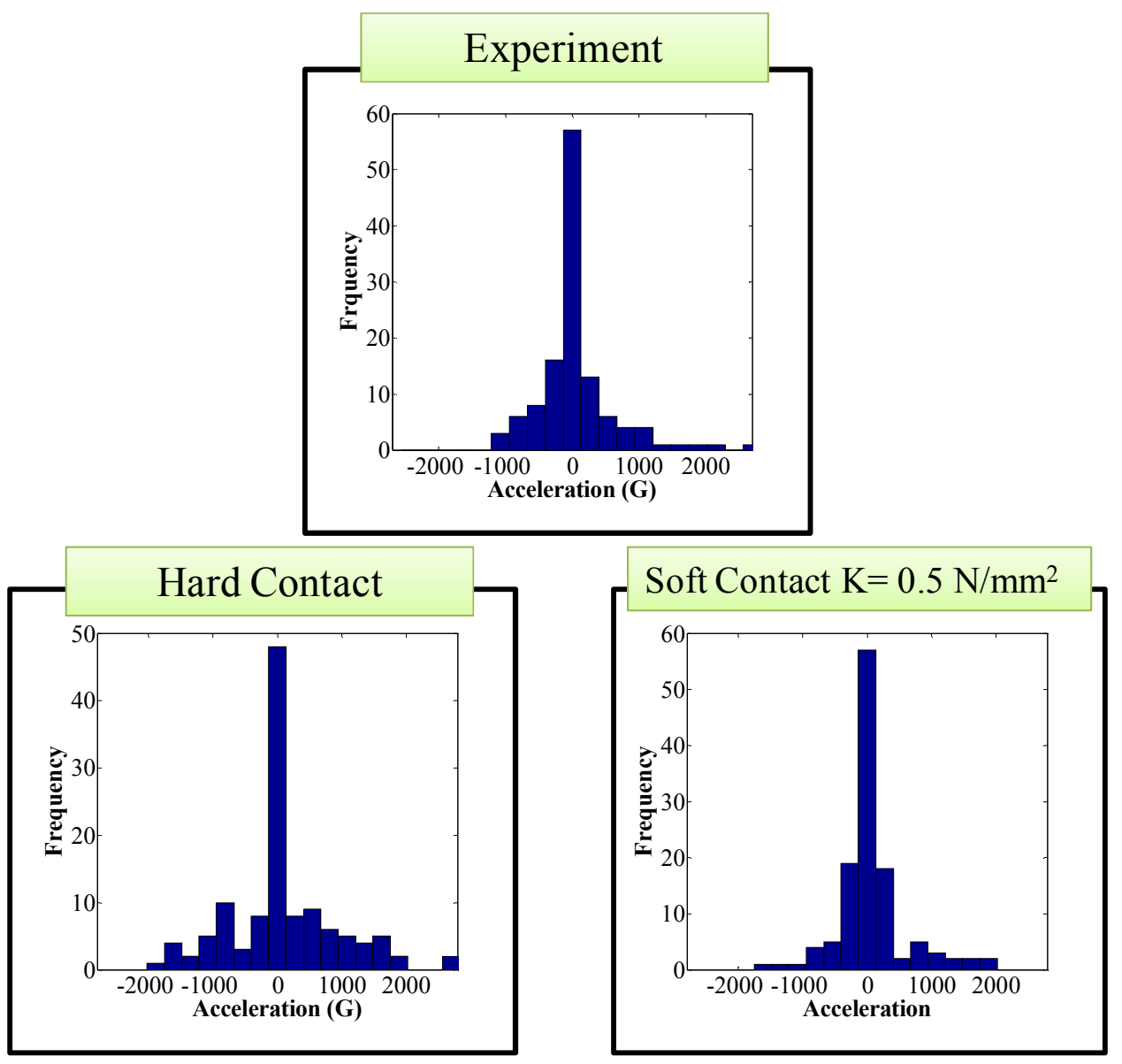

Figure 3-34- Comparison of the experimental acceleration histogram with those of the FEA models with soft and hard contact properties.

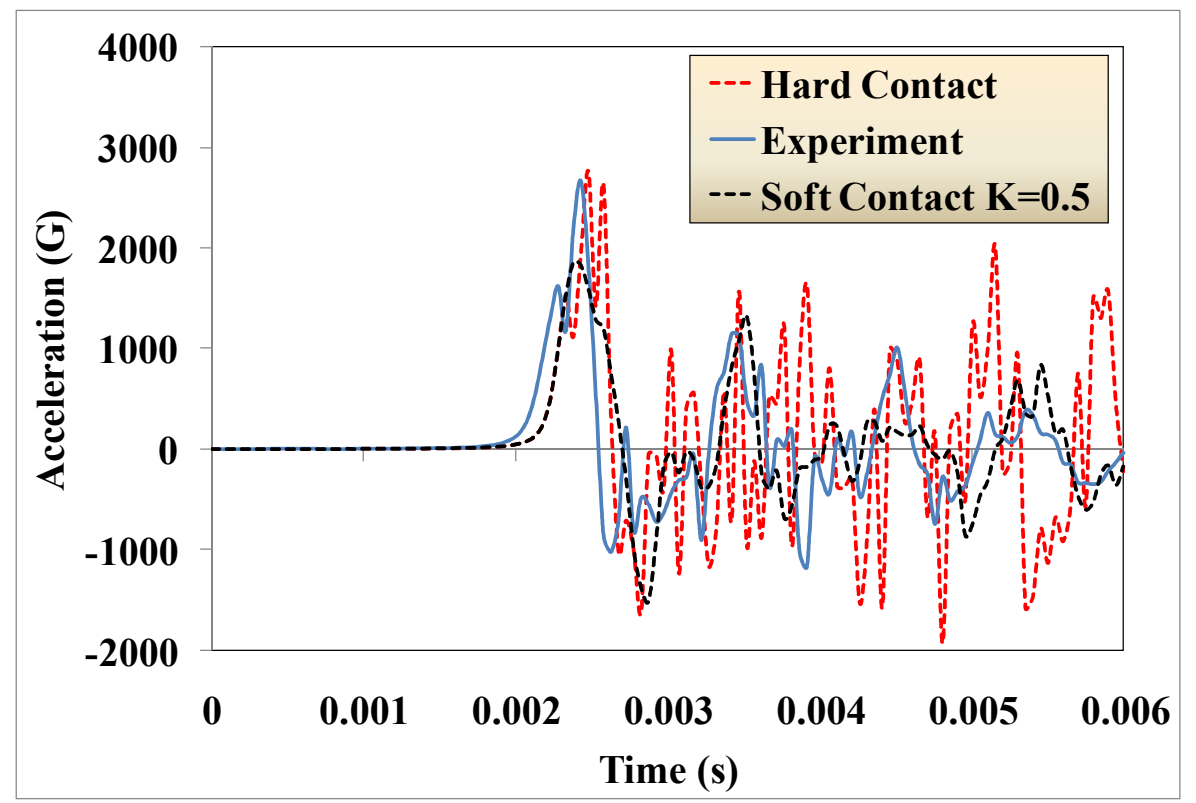

Figure 3-35- Comparison of the experimental acceleration response with those of the FEA models with soft and hard contact properties. 
On the other hand, comparison of the experimental strain histogram with those of obtained by modeling soft and hard contact show no significant reduction in strain when soft contact is used rather than hard contact. These results suggest that the acceleration measured next to the post correlates better with the post contact stress than does the strain amplitude relatively far away from the post. In other words, the contact between the internal components is causing localized plastic deformation at the point of contact, but is not having a dramatic effect on the overall global deflections of the PWB.

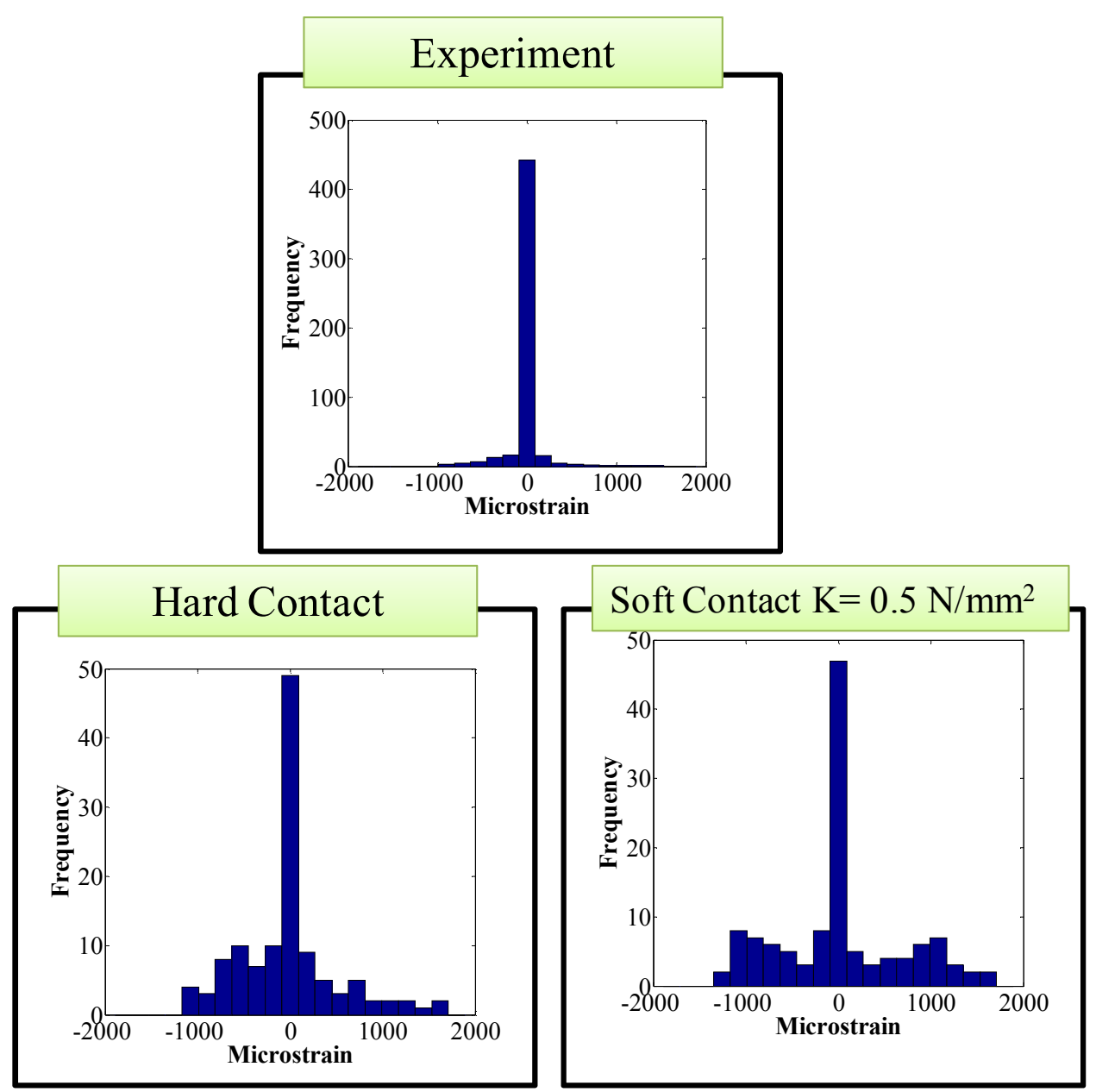

Figure 3-36 Comparison of the experimental strain histogram with those of the FEA models with soft and hard contact properties. 


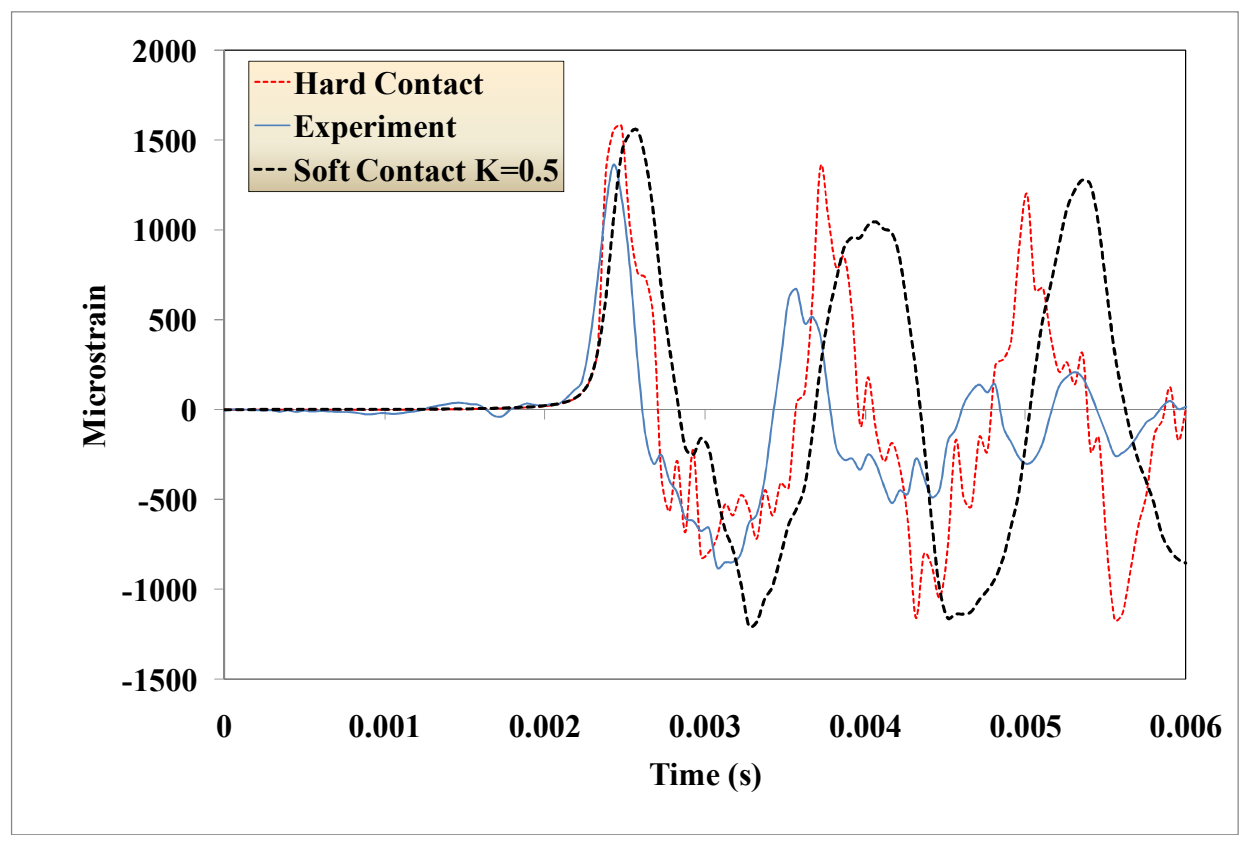

Figure 3-37- Comparison of the experimental strain response of the PWB with those of the FEA models with soft and hard contact properties.

Now that is established that modeling soft contact help predict the dynamic response of the full product more efficiently, a parametric study is conducted to find the optimal contact stiffness. In the first step of the parametric study, no damping is defined and the contact stiffness is varied according to Error! Reference source not found..

Table 3-1- Illustrates the effect of contact stiffness on the range of the first cycle of the strain history. Strain is measured at point in the 1 direction as shown in Figure 2-47.

\begin{tabular}{|c|c|c|c|c|c|c|c|}
\hline $\begin{array}{l}\text { Contact Stiffness } \\
\qquad\left(\mathrm{N} / \mathrm{mm}^{2}\right)\end{array}$ & 0.5 & 2 & 5 & 10 & 20 & 50 & Experiment \\
\hline Strain Range $\mu \varepsilon$ & 2752 & 2649 & 2609 & 2584 & 2586 & 2638 & 2241 \\
\hline
\end{tabular}

Figure 3-38 illustrates how the slope of the pressure-overclosure curve affects the range of the first cycle of the strain history obtained from the simulation. Strain is 
measured at point 2 in the " $x$ " direction as shown in Figure 2-47. The results of the parametric studies of the contact mechanics indicate that as the contact stiffness increases from $0.5 \mathrm{~N} / \mathrm{mm}^{2}$ to $50 \mathrm{~N} / \mathrm{mm}^{2}$, the normalized strain RMS with respect to hard contact decreases 10\% (Refer to Figure 3-39). On the other hand, the cyclic range of the first strain cycle varies non-monotonically with the closest agreement with experiment occurring at $10 \mathrm{~N} / \mathrm{mm}^{2}$ (15\% higher than the experimental value). Therefore $10 \mathrm{~N} / \mathrm{mm}^{2}$ is chosen as the optimal contact stiffness value. On the other hand, both the acceleration normalized RMS and the acceleration range of the first cycle increase as the contact stiffness increases. In the modeled product, the acceleration RMS error value increased by $49 \%$ and the acceleration range of the first cycle increased by $50 \%$, as the contact stiffness increased from $0.5 \mathrm{~N} / \mathrm{mm}^{2}$ to 50 $\mathrm{N} / \mathrm{mm}^{2}$ (Refer to Figure 3-40).

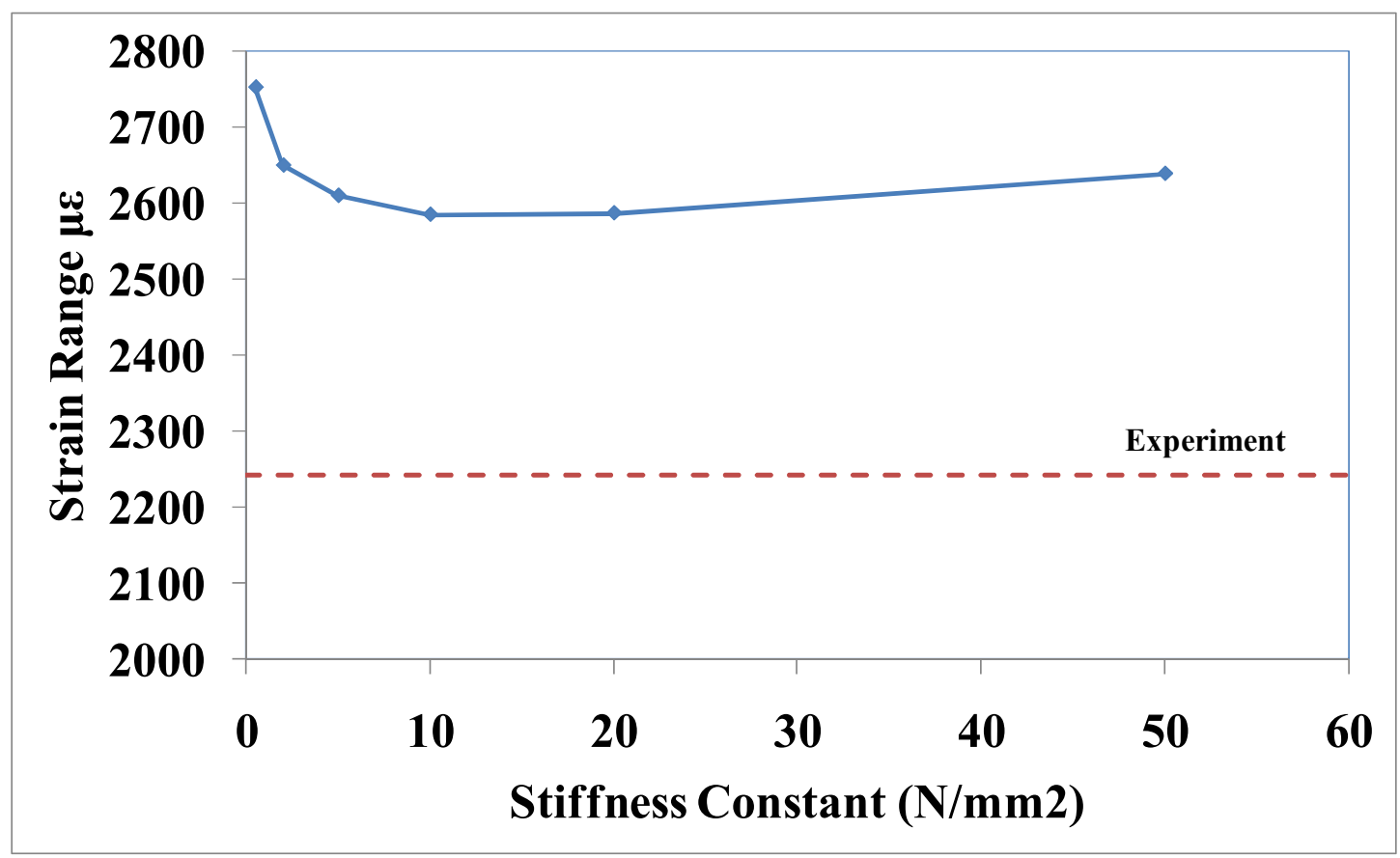

Figure 3-38 Illustrates the effect of contact stiffness on strain range variation, calculated from the first peak and valley of strain profiles. Strain is measured at point in the 1 direction as shown in Figure 2-47. 


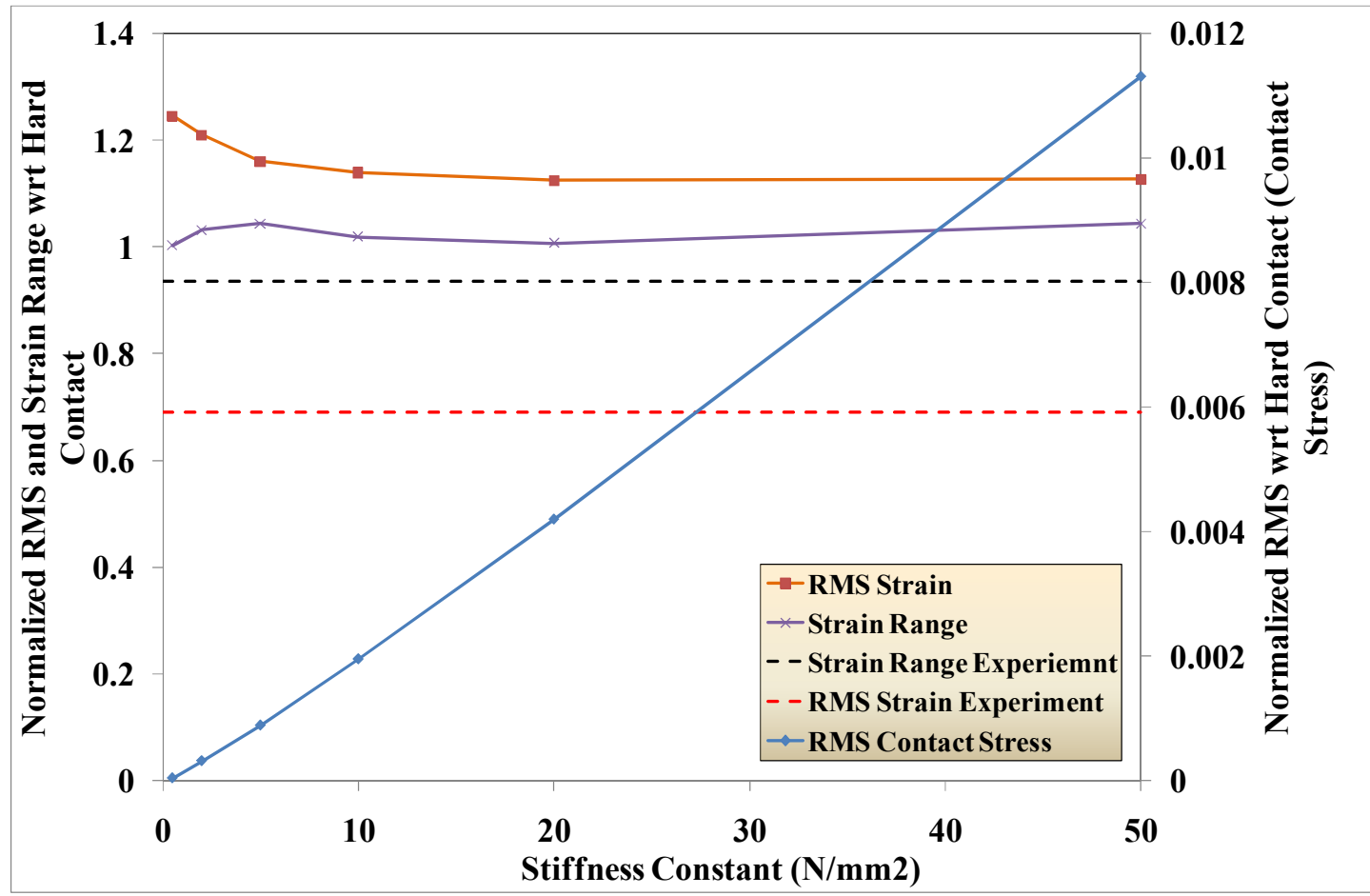

Figure 3-39 Illustrates the normalized RMS strain and the strain range values based on various contact stiffness. Strain is measured at point 2 in the $x$ direction as shown in Figure 2-47. RMS and Strain range values are normalized with respect to hard contact.

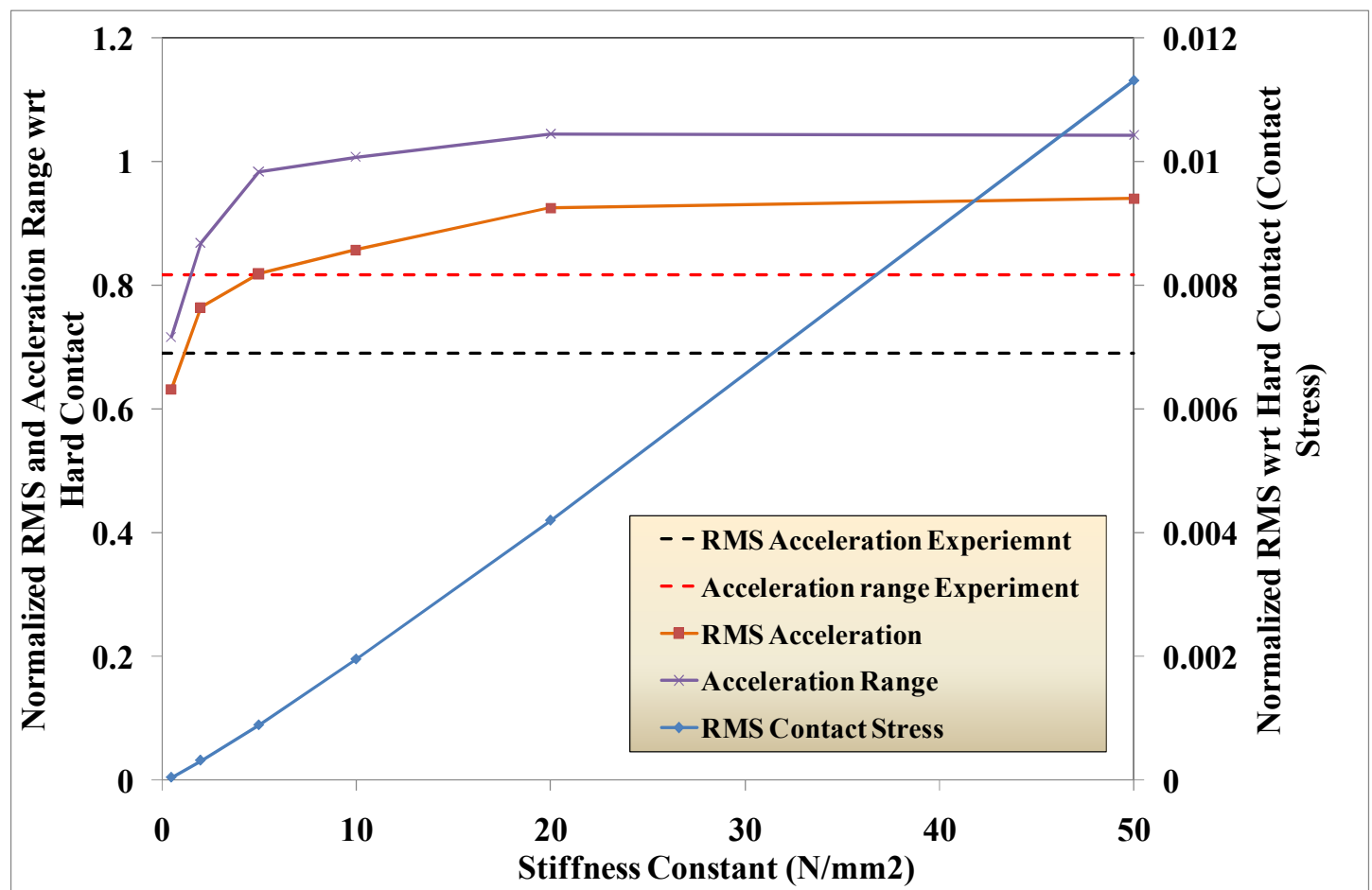

Figure 3-40 Illustrates the normalized RMS acceleration and the acceleration range values based on various contact stiffness. RMS and acceleration range values are normalized with respect to hard contact. Acceleration is measured on the PWB according to Figure 2-18. 
In the next step of the parametric study, the contact stiffness is kept constant at $10 \mathrm{~N} / \mathrm{mm}^{2}$, while the fraction of critical damping is defined in the contact property module and varied according to Error! Reference source not found..

Table 3-2 Illustrates how strain range of the first cycle of the strain history varies as a function of critical damping fraction. Strain is measured at point in the 1 direction as shown in Figure 2-47.

\begin{tabular}{ccccccc} 
Fraction of & undamped & $\mathbf{0 . 8}$ & $\mathbf{2}$ & $\mathbf{8}$ & Experiment \\
Critical Damping & & & & & \\
& & & & & \\
\hline Strain Range $\boldsymbol{\mu \varepsilon}$ & 2584 & 2463 & 2486 & 2588 & 2241 \\
\hline
\end{tabular}

The results indicate that the strain RMS value decreased monotonically by $22 \%$ as the damping was increased from 0 to 8 times the critical damping. However, the first-cycle strain-range varied non-monotonically and was closest to the experimental value ( $9 \%$ higher than the experimental value) when the fraction of critical damping was set to 0.8 . Therefore, this value is selected as the optimal damping level for this structure. The corresponding acceleration RMS was nonmonotonic, with a minimum at around critical damping ratio of 2 . On the other hand, the acceleration range of the first cycle decreased monotonically by $10 \%$ over the range of damping examined. 


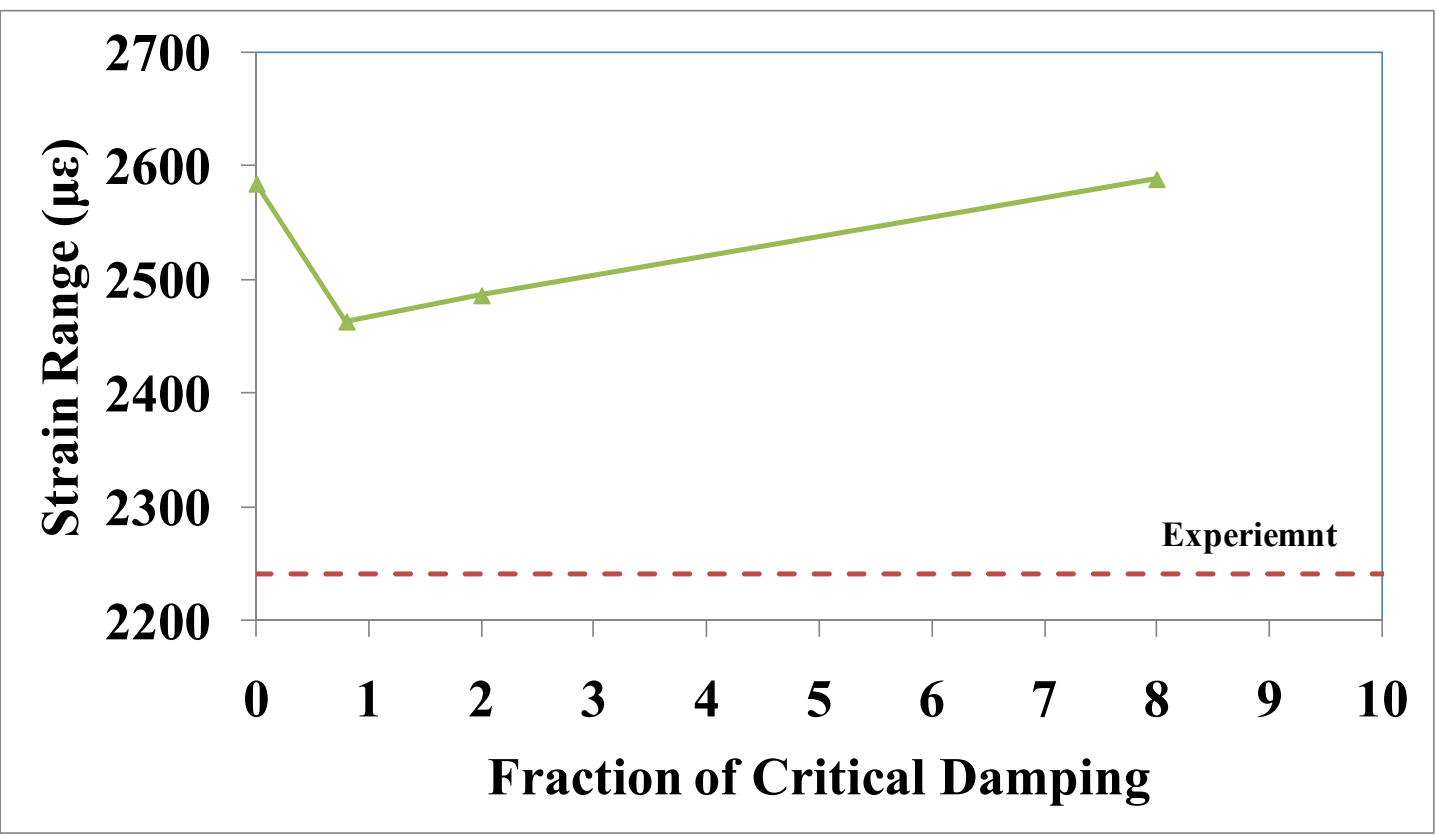

Figure 3-41 Illustrates the effect of fraction of critical damping on strain range of the first cycle. Strain is measured at point 2 in the $x$ direction as shown in Figure 2-47.

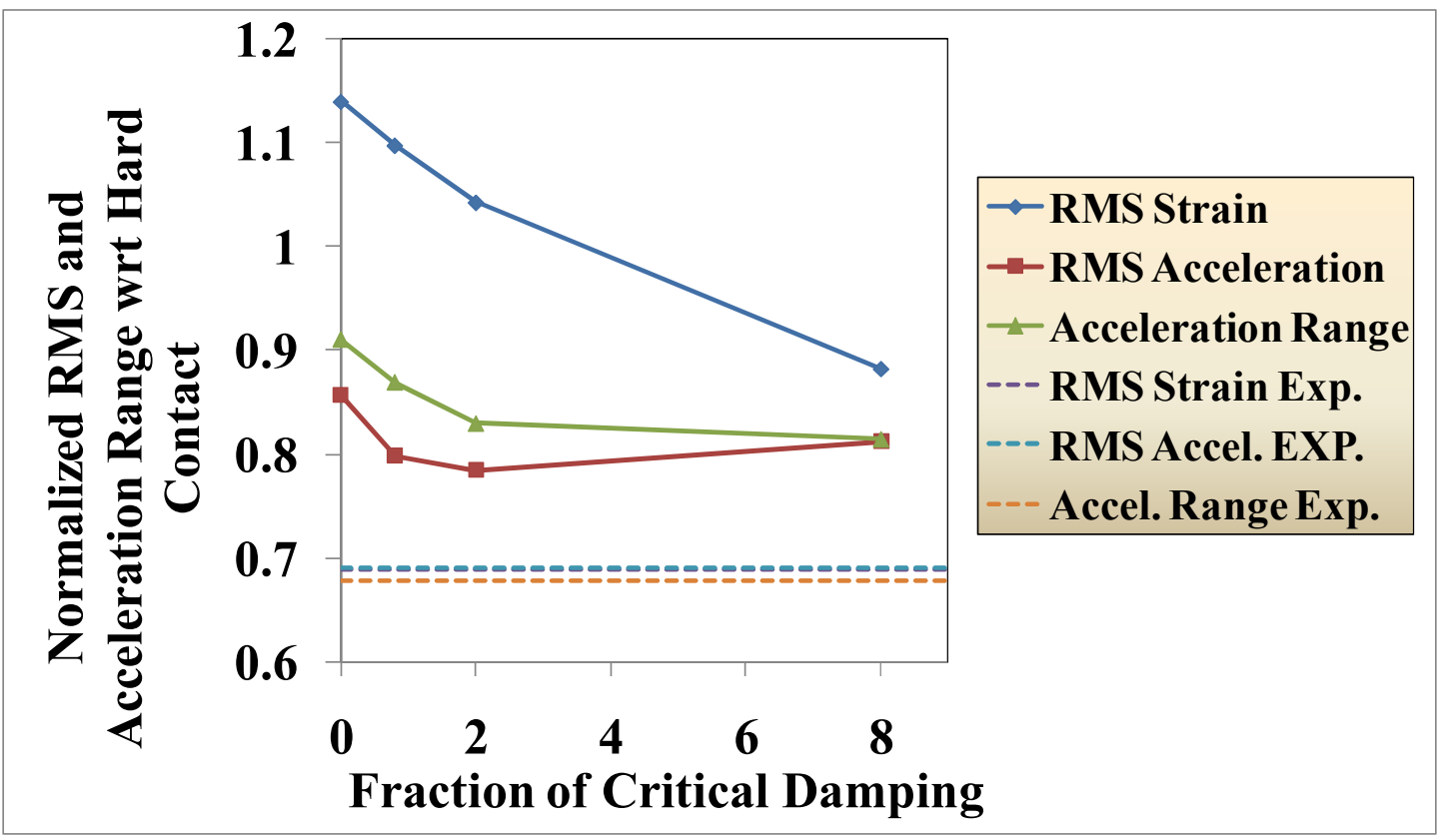

Figure 3-42- Illustrates the RMS of the strain and acceleration profiles, as well as the acceleration range of the first cycle based on various fraction of critical damping values. All the values are normalized with respect to hard contact.

The last step of the parametric study is to increase the mass-proportional Raleigh damping factor, $\alpha$, to achieve a better agreement between experimental and 
simulation results. It was established in the preceding sections that $\alpha$ is highly structure and load dependent. As a result, the $\alpha$ values of the PWB and the plastic housing, obtained from modal analysis will be different when individual components of the full product are assembled together. This is due to an increase in the number of the constraints and the level of loading imposed on the individual components in the full product assembly.

Therefore, the Raleigh damping factors, $\alpha$, of the PWB and the plastic housing were parametrically increased by $300 \%$ and $500 \%$. The results indicate that both the strain RMS value and the error in the strain range of the first cycle decreased when the Raleigh damping factor, $\alpha$, was increased. The errors in the strain range for the first cycle and the RMS strain value both decreased monotonically over this range. Similarly, the acceleration range of the first cycle decreased by $7 \%$ and the acceleration RMS value decreased by $10 \%$ over this range. The optimal Raleigh damping factors in the final assembly were therefore chosen to be 5 times that obtained by testing the PWB and housing individually. 


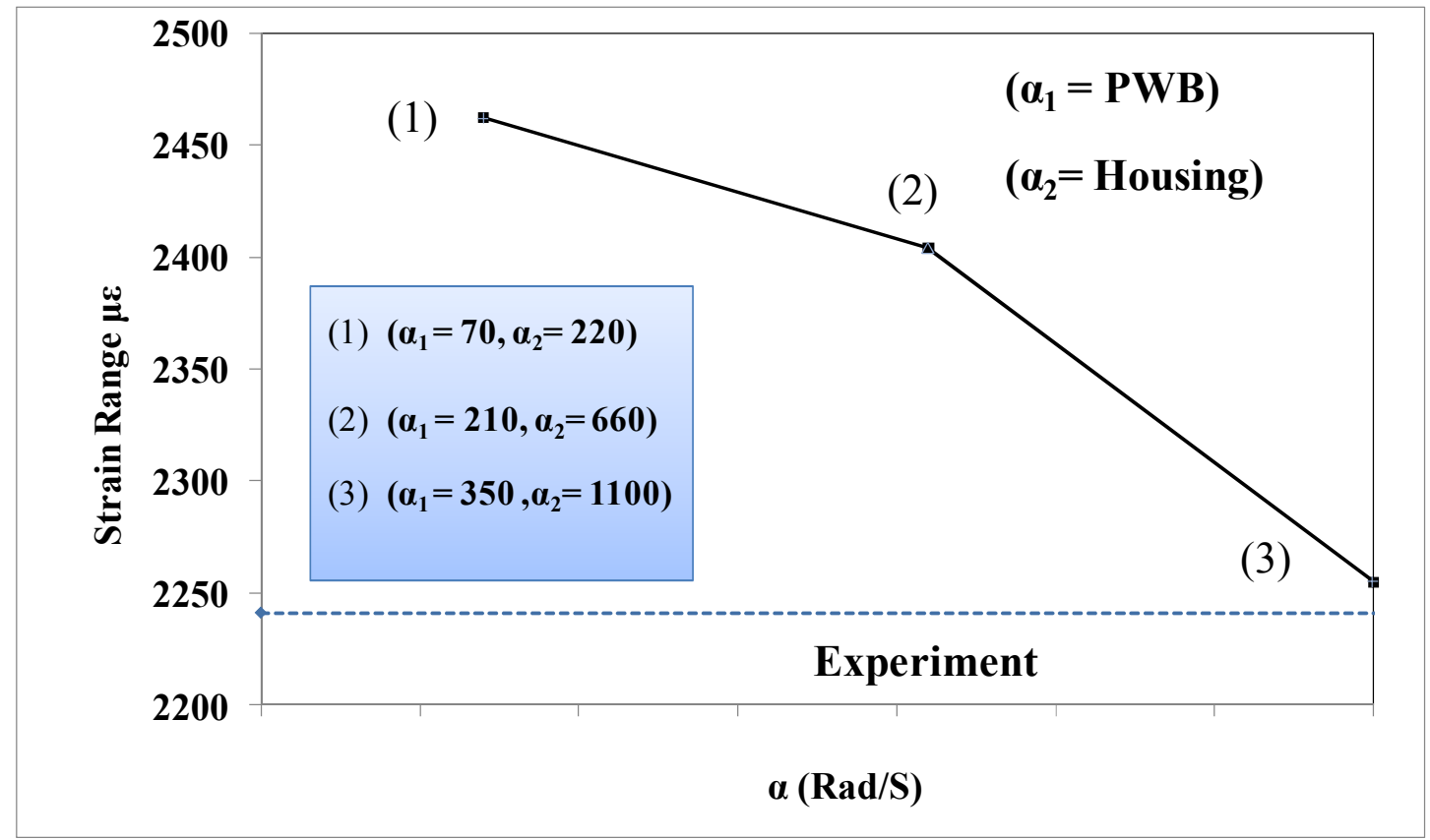

Figure 3-43 Illustrates the effect of Raleigh damping on strain range of the first cycle. Strain is measured at point 2 in the $x$ direction as shown in Figure 2-47. The Raleigh damping factors $\alpha_{1}, \alpha_{2}$ belong to the PWB and the plastic housing respectively.

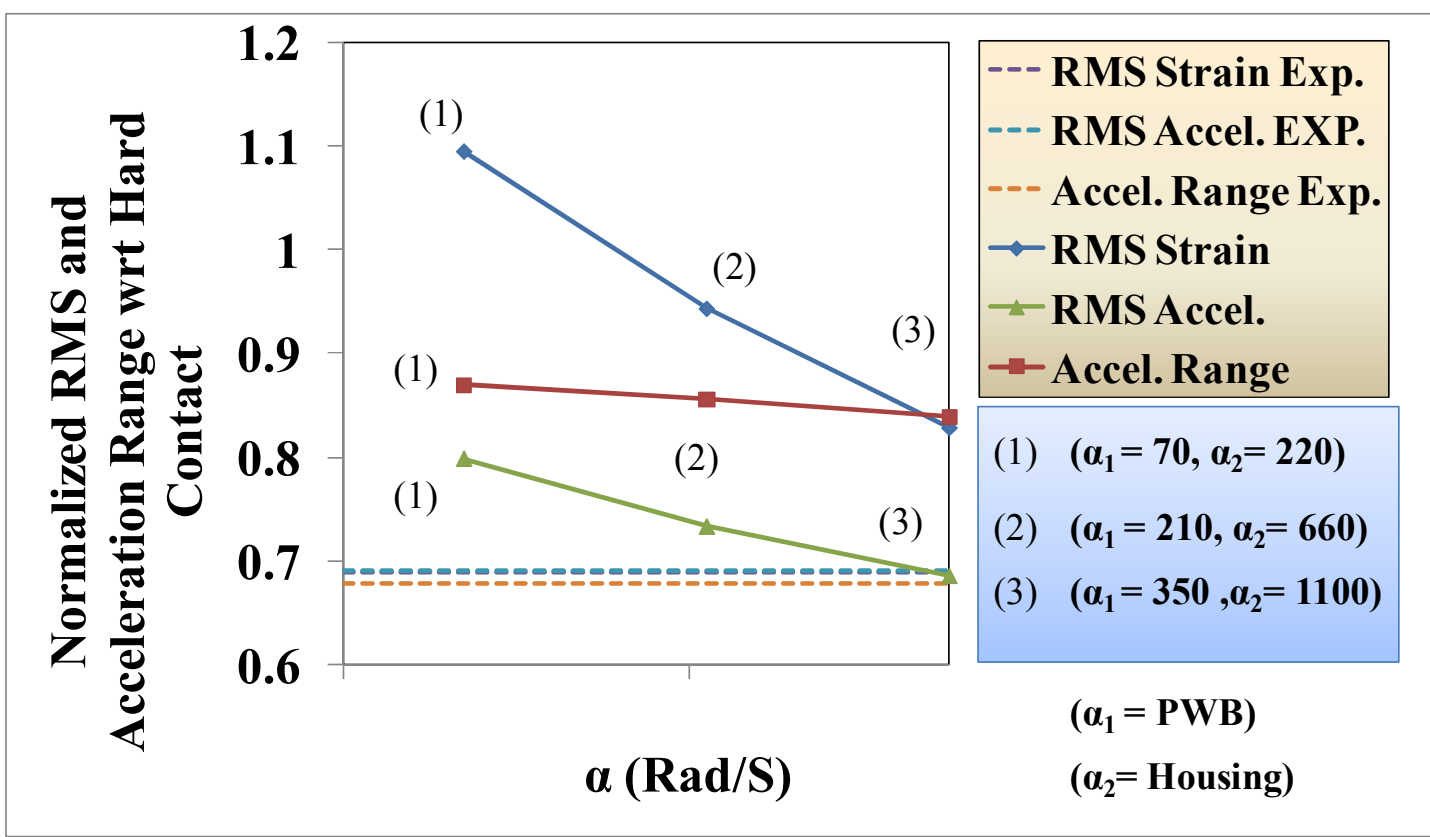

Figure 3-44 Illustrates the acceleration and strain RMS based on various Raleigh damping values. The Raleigh damping factors $\alpha_{1}, \alpha_{2}$ belong to the PWB and the plastic housing respectively.

Figure 3-45 to Figure 3-48 compares the FEA results, obtained using the optimized Raleigh damping and mechanical contact property parameters, to that of the experiment. In these figures strain is measured at point 2 as shown in Figure 2-18 
and acceleration is measured on the PWB also shown in Figure 2-18. Figure 3-45 illustrates that there is a fairly good agreement between the first peak of strain profiles, as well as the strain range of the first cycle. However, it seems that the PWB in the actual test damps out much faster than the FEA model. According to Figure $3-46$, the first peak of the FEA acceleration is underestimated by $10 \%$ but the first valley of the simulation result is much higher than that of the experiment and consequently results in a higher acceleration range for the model. It is possible that the strain gage wires, routed inside the device, restrict the motion of the PWB inside the full product. These wires are not incorporated in the FEA model, hence a higher acceleration is predicted for the valley of the first cycle of the acceleration profile. It should be noted that for reliability analysis purposes, the focus should be on the time history of strain and acceleration profiles rather than the frequency response functions of the strain and acceleration measurements to drop loading. However, FFT graphs provide good clues on how the FEA model can be improved to obtain a better agreement between simulation and experiment results. The FFT graphs shown in Figure 3-47 and Figure 3-48 suggest there is a slight change in the natural frequencies of the FEA model compared to that of the experiment. On the other hand, there is a significant difference in the amplitudes of the natural frequencies. This discrepancy, apart from complexity of the system, as well as the low resolution of the FFT graphs, is possibly due to the fact that in the FEA model, there is only one global damping defined, whereas in reality each natural mode requires its own damping parameter. 


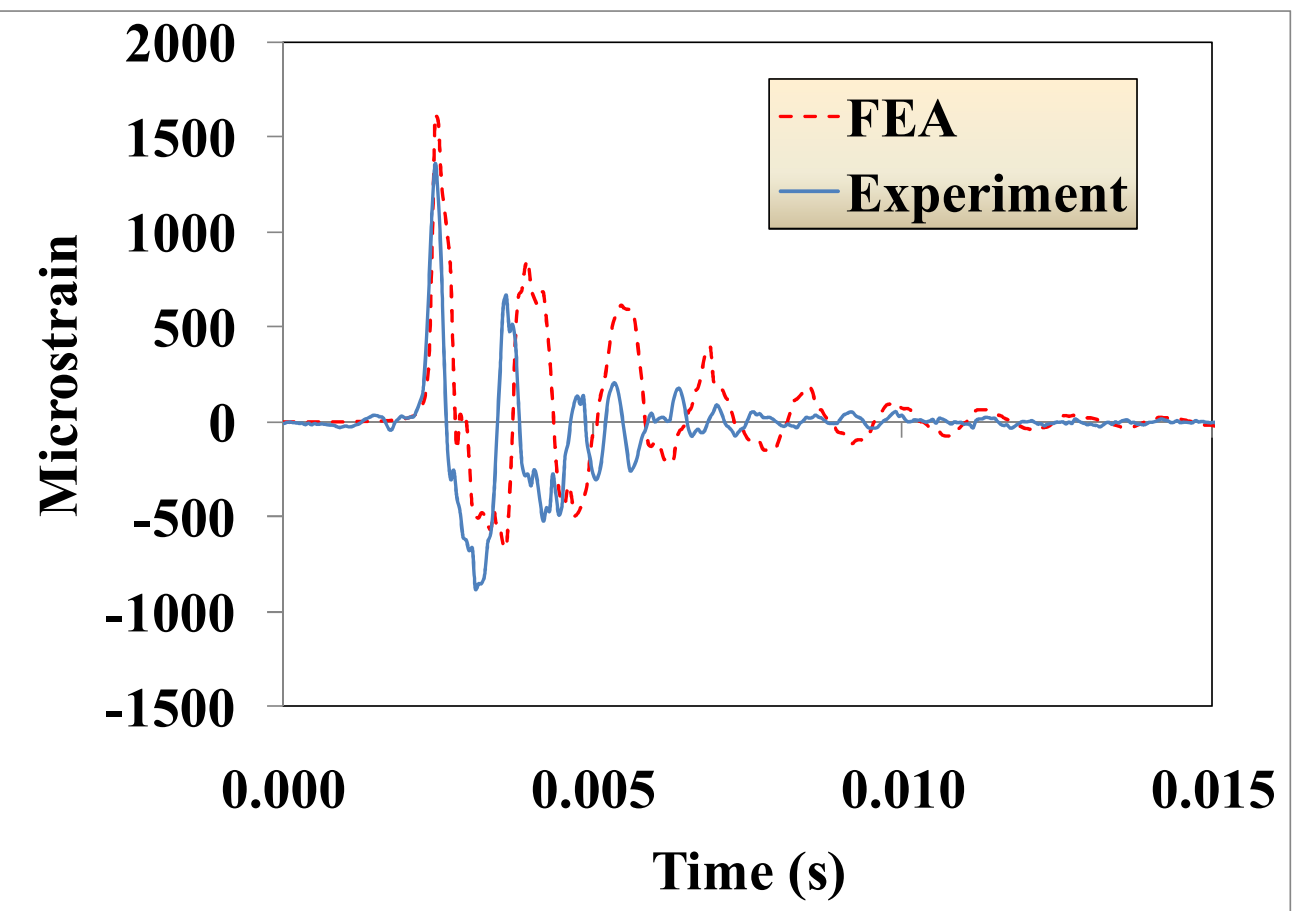

Figure 3-45 Drop test FEA results VS. Experiment results- Strain is measured at point 2 in the "1" direction as shown in Figure 2-47.

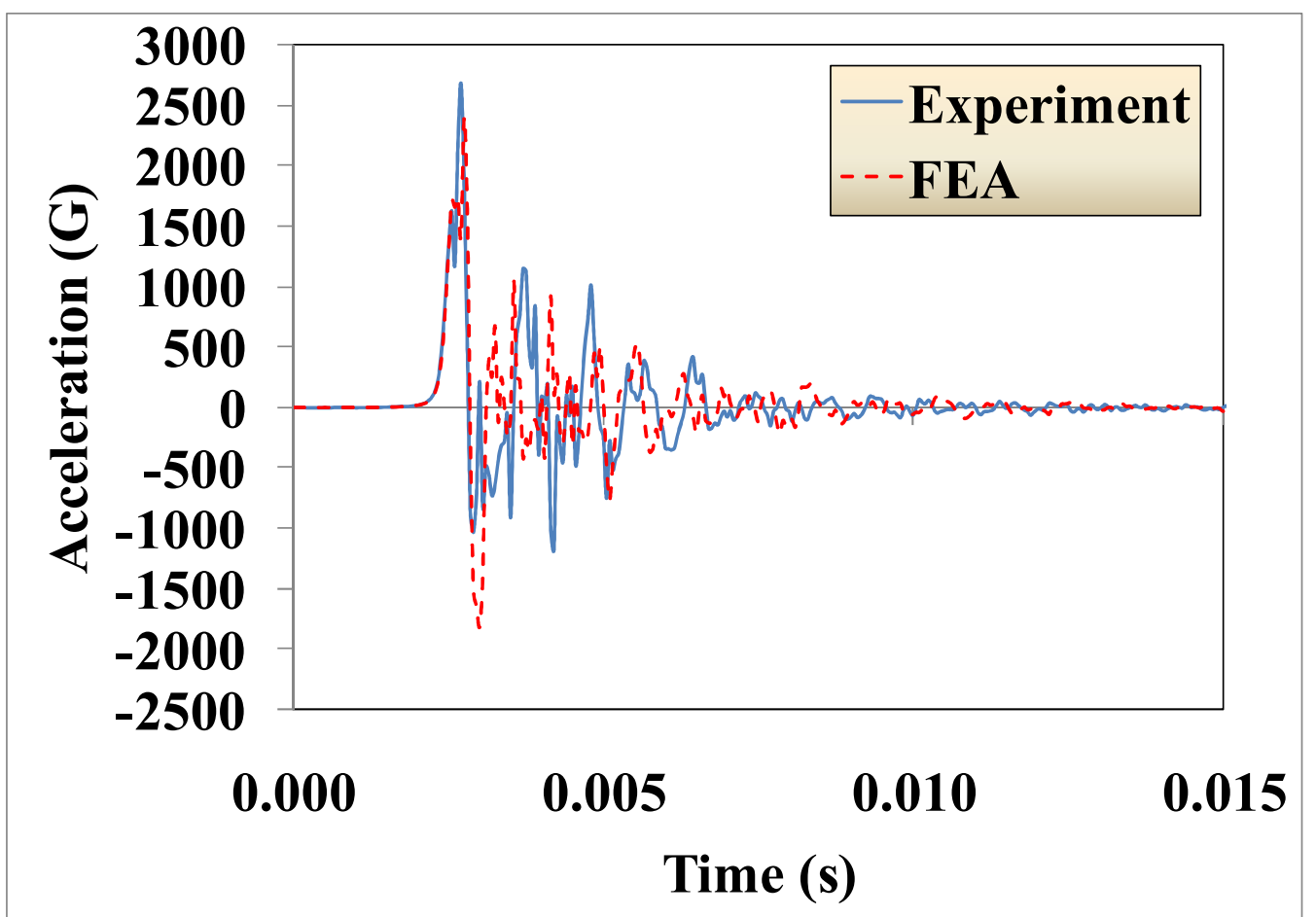

Figure 3-46 Drop test FEA results VS. Experiment results. Acceleration is measured on the PWB in the outof-plane direction. Refer to Figure 2-18 for exact location of the Accelerometer. 


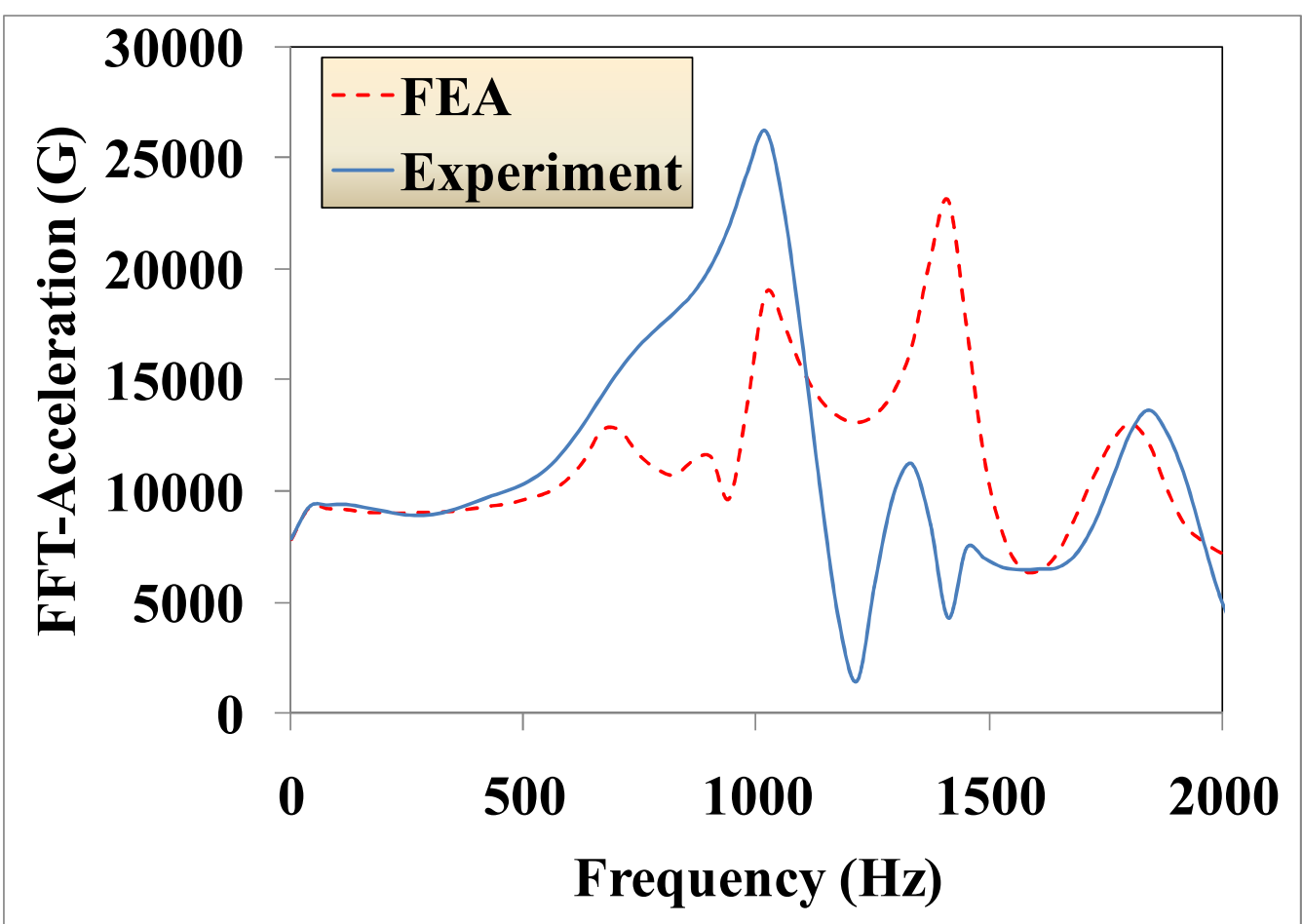

Figure 3-47- FTT response of the accelerometer on the PWB shown in Figure 2-18 vs. FEA results.

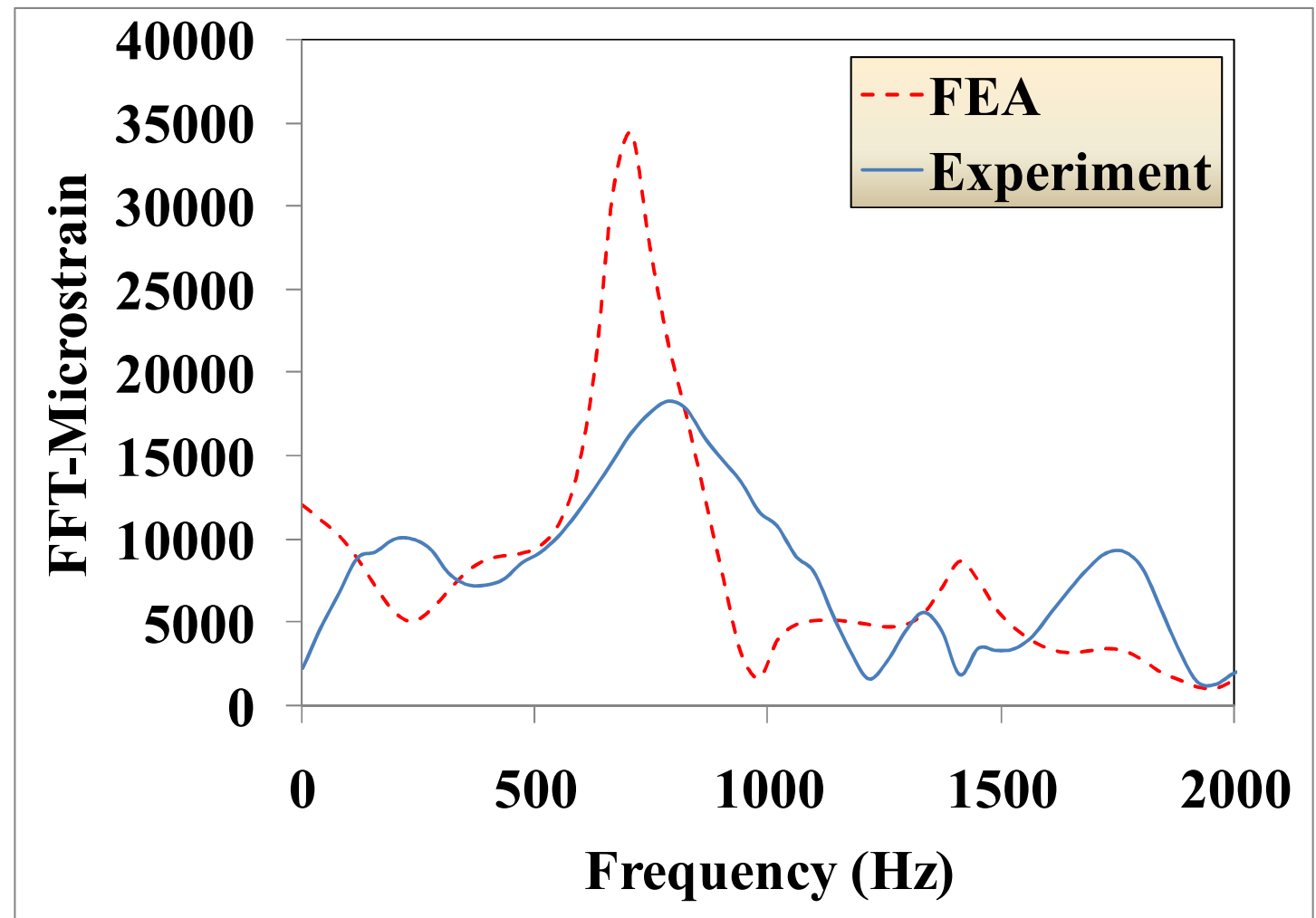

Figure 3-48 FTT response of the strain gage at point 2 as shown in in Figure 2-47 vs. Experiment results. 


\section{$\underline{3.6 \text { Discussion }}$}

The objective of this study was to investigate the ability of finite element models to accurately predict the transient response of complex portable electronic devices for shock and drop loading. Finite element modeling is carried out using a progressive hierarchical multi-step technique in which material properties and boundary conditions of individual subassemblies are calibrated from simple shock and vibration tests prior to proceeding to drop simulation of the full product. It is shown in this study that, the validated modulus of elasticity and damping parameters obtained from the modal test and simulation can precisely capture the dynamic response of the PWB both in drop and shock simulations. This signifies the importance of performing modal analysis prior to shock and drop simulation. The results of drop and shock simulation of the full product suggest that the high acceleration levels in the FEA model are resulted from impact between the internal parts. However, these impact-induced high accelerations are not seen in the actual product shock and drop tests, because the contact surfaces are not elastic and in reality plastic deformation occurs due to high acceleration levels. To truly capture the contact stresses and the impact decelerations correctly, the contacts must be modeled with suitable damping and non-conservative behavior. In this study, a parametric study was conducted on the various available parameters of contact property module in ABAQUS. The goal was to find out how contact property parameters need to be changed to effectively simulate the effects of plastic deformation for elastic materials. 


\section{Chapter 4 Summary}

The main conclusions of this thesis as well as the contributions of this project are presented and discussed here, along with recommendations for future work.

\subsection{Conclusions and Discussions:}

This thesis explores a systematic approach for modeling the dynamic response of complex portable electronic devices to highly non-linear excitations such as shock and drop loading. A testing program was first described in Chapter 2 of this thesis, to provide a comprehensive experimental understanding of the dynamic behavior of the product under study and its subassemblies. The product is a portable electronic device consisting of an organic laminated circuit card assembly in a plastic clam-shell welded housing. Various broad band, shock, and drop tests were conducted on the product and on its individual sub-assemblies (PWB and housing), to identify relevant material properties, structural nonlinearities, boundary conditions and loading conditions. Chapter 3 utilizes the experimental results obtained in Chapter 2 to explore adequate methods to develop finite element models of the product under study. FEA modeling methods for drop simulation are very important in the design of portable electronic products because FEA models can be very useful to detect failure modes under drop loading and to optimize and ruggedize the design early in the design phase before the product is built.

The challenge in system-level modeling for drop testing is that discrepancies between simulation and experimental results are tremendously difficult to trace back to the source of the problem. Therefore to facilitate the troubleshooting process the 
whole device was divided into its constituent 'building blocks'. Subsequently, various broad band, shock, and drop tests were performed on each component and subassembly of the product, prior to the full product drop testing. The goal is to identify and isolate some of the nonlinearities involved in drop testing by comparing against the results of simple shock and broad band tests on the subassemblies. Initially, broad band tests were performed on the subassemblies of the product because the vibration tests are well suited to first estimate the modulus of elasticity as well as the damping parameters of the constituent components of the system, prior to performing shock and drop simulation of the full product. In the next step, the modulus of elasticity, as well as the damping parameters, which were obtained in the broad band test, were validated by performing shock simulations on the PWB. Finally, the validated modulus of elasticity and damping parameters were used as inputs for conducting drop simulation of the entire product. The central goal is to investigate simple FEA techniques for capturing the dynamic response of the device under study.

The results of shock and drop simulation of the full product show that when there are clearances between internal parts, FEA models with linear elastic material properties predict unrealistically high accelerations due to elastic impact between neighboring parts. However, these impact-induced high accelerations are not seen in experiments, because in reality the contact dynamics are not elastic and plastic deformation at the contact surface may reduce the acceleration levels. Therefore, when using linear elastic material properties in FEA models realistic estimates of the contact stresses and the impact decelerations require the use of suitable non- 
conservative features such as 'soft' contact, contact damping, and contact friction. In this study, a parametric sensitivity study was conducted to explore the various methodologies available in ABAQUS for modeling non-conservative contact. The goal was to find out how contact property parameters and damping parameters need to be defined to effectively simulate the effects of plastic deformation and contact friction and other dissipation mechanisms, using models with elastic material properties.

The accuracy of the drop modeling assumptions are assessed by comparing two important parameters between the model and experiments: the cyclic range of the first response cycle after the drop impact, as well as the RMS error over the entire time history of the record. Although these metrics are monitored for both the acceleration response as well as the strain response at key locations on the PWB inside the plastic housing, priority is given to the errors in the strain prediction. This is due to the fact that flexural strain is one of the leading drivers for most failures on PWAs under drop loading. Additionally, matching the cyclic range of the first strain cycle also has priority over matching the RMS value, because roughly $90 \%$ of the drop damage occurs during the first cycle.

The conclusions from the experimental and simulation tasks in this study are summarized below:

- The responses of the product can be examined only up to $2000 \mathrm{~Hz}$ because the fixture used in the broad band and drop test of the full product (and also of the empty plastic housing) has fundamental resonant frequency at around $2000 \mathrm{~Hz}$. Similarly, the cantilever beam used to identify the elastic modulus of the plastic 
material used in the housing is only characterized up to $800 \mathrm{~Hz}$ because of the first resonant frequency of the cantilever fixture used in this test.

- In shock test on clamped PWB, the acceleration magnification was constant for excitation levels less than $40 \mathrm{Gs}$ and it increased as acceleration increased beyond 40Gs. This is probably due to nonlinearities in the system.

- Adding contact springs under the charge contact pads of the PWB increased the acceleration magnification factor of shock response by $7 \%$ compared to just simple support at the two locator holes. Mounting the PWB in the product reduced the acceleration magnification factor of shock response by $25 \%$, compared to just simple support at the two locator holes. This could be due to attenuation of some of the shock energy by the plastic housing in the product level shock test.

- Conversely, mounting the PWB in the product increased the acceleration magnification factor of drop response by $20 \%$, compared to just simple support under the two locator holes in the PWB. The higher accelerations in the product are believed to be as a result of impact between the PWB and the housing at various contact points due to lack of sufficient clearance.

- In the shock test of the clamped PWB, the first natural frequency of the PWB is found to coincide with the first notch of the shock spectrum of the fixture on which the board sits. Therefore, the first mode of the PWB is not excited and consequently, the second natural mode of the PWB is the dominant natural mode. However, in the shock test of the spring-mounted PWB, as well as in the drop test of the clamped PWB, the notches of the shock spectrum of the input 
excitation did not coincide with the first natural frequency of the PWB. Therefore, as expected, the first natural mode was the dominant mode.

- The shock 'relaxation' typically observed in shock testing with electro dynamic shakers is found to cause low frequency perturbations of the shock spectrum, causing additional notches in the shock spectrum that can filter out the low frequency response of the system.

- Response frequencies of the PWB in the full product match reasonably well in both broad band and drop tests, within the measurement resolution $( \pm 40 \mathrm{~Hz}$ ). Generally, the frequency response of a non-linear system is expected to change with the level and type of loading. In this case, the effects of non-linearities do not appear to be strong enough to be detectable with our measurement resolution.

- The modulus of elasticity and damping parameters obtained from the random vibration test and FEA modal analysis were found to provide reasonably good estimates of the dynamic response of the PWB in shock. However, in drop simulations the damping parameter needed be increased.

- Raleigh damping parameters obtained for individual components of the system such as the PWB and the housing are found to be dependent on the structural boundary constraints and therefore do not predict the assembly response very well. This brings attention to the fact that defining Raleigh damping is not the most efficient way to represent system damping and more effective methods are needed to model damping mechanisms in complex assemblies.

- The results of shock and drop simulation of the full product show that the FEA models predict unrealistically high accelerations due to elastic impact between 
the internal parts. However, these impact-induced high accelerations are not seen in the experiment, because in reality the contact dynamics are not elastic and plastic deformation at the contact surface may reduce the acceleration levels.

- The results of the parametric studies of the contact mechanics indicate that as the contact stiffness increases from $0.5 \mathrm{~N} / \mathrm{mm}^{2}$ to $50 \mathrm{~N} / \mathrm{mm}^{2}$, the strain RMS error decreases $25 \%$. On the other hand, the cyclic range of the first strain cycle varies non-monotonically with the closest agreement with experiment occurring at 10 $\mathrm{N} / \mathrm{mm}^{2}\left(15 \%\right.$ higher than the experimental value). Therefore $10 \mathrm{~N} / \mathrm{mm}^{2}$ is chosen as the optimal contact stiffness value. On the other hand, both the acceleration RMS error and the acceleration range of the first cycle increase as the contact stiffness increases. In the modeled product, the acceleration RMS error value increased by $73 \%$ and the acceleration range of the first cycle increased by $50 \%$, as the contact stiffness increased from $0.5 \mathrm{~N} / \mathrm{mm}^{2}$ to $50 \mathrm{~N} / \mathrm{mm}^{2}$.

- In next step of the parametric study, contact damping was added to the model of the product. The results indicate that the strain RMS error value decreased monotonically by $7 \%$ as the damping was increased from 0 to 8 times the critical damping. However, the first-cycle strain-range varied non-monotonically and was closest to the experimental value ( $9 \%$ higher than the experimental value) when the fraction of critical damping was set to 0.8 . Therefore, this value is selected as the optimal damping level for this structure. The corresponding acceleration RMS error was non-monotonic, with a minimum at around critical damping ratio of 2 . On the other hand, the acceleration range of the first cycle decreased monotonically by $10 \%$ over the range of damping examined. 
- In the last step of the parametric study, the Raleigh damping factors, $\alpha$, of the PWB and the plastic housing were parametrically increased by $300 \%$ and $500 \%$. The results indicate that both the strain RMS error value and the error in the strain range of the first cycle decreased when the Raleigh damping factor, $\alpha$, was increased. The errors in the strain range for the first cycle and the RMS strain error both decreased monotonically over this range. Similarly, the acceleration range of the first cycle decreased by $7 \%$ and the acceleration RMS error value decreased by $10 \%$ over this range. The optimal Raleigh damping factors in the final assembly were therefore chosen to be 5 times that obtained by testing the PWB and housing individually.

\subsection{Contribution of Thesis}

- Presented a systematic and comprehensive, process to gain insights into the drop response of complex assemblies. The method is based on hierarchical modeling and testing of the dynamic response of each major sub-assembly in the product under progressively more severe conditions such as broad-band modal analysis, shock loading and drop loading.

- Presented several guidelines for testing and simulation of the dynamic response of complex portable electronic assemblies subjected to shock and drop loading, based on systematic parametric studies:

- Quantified the importance of studying the effects of pulse duration on the dynamic response of a system. 
- Demonstrated the inadequacy of the Raleigh damping factors when a progression is made from subassembly-level to assembly-level simulation.

- Conducted parametric sensitivity studies to explore the various methodologies available in ABAQUS for modeling "soft" nonconservative contact between internal parts in complex assemblies.

\subsection{Future Work}

The following suggestions for future work are outlined below:

- In this thesis, product drop test was conducted only in one orientation. The heavy battery for this portable device was assumed to not affect the PWB response for this particular orientation. Therefore, additional drop testing, as well as finite element modeling are needed for drop along other orientations, to study the effect of battery mass on the PWB response. Additionally, there is a need to perform shock and drop tests and simulations on a fully populated PWB, to study the effects of components on the dynamic response of the PWB.

- The PWB and other components of the product are likely to experience severe local plastic deformation at the contact interfaces and constraint locations during drop testing. Therefore, another area that needs further research is computationally effective on method to incorporate localized plasticity in the finite element models.

- Identification of the correct mode shapes and the corresponding frequencies was important in various parts of the study. Multiple accelerometers had to be 
placed on the device at various locations to obtain the frequency response function, as well as the phase plots between the accelerometers. Since the mass of these accelerometers perturb the modal response, non-contact methods such as laser vibrometer should be explored for more accurate vibration measurements.

- The metric used to assess the accuracy of the simulations in the parametric studies was the strain range of the first cycle of the drop response. A more useful metric in future studies will be the total damage per drop based on integration of some damage law over the entire drop response history. 


\section{Appendix A Dimensions of the Product}

All dimensions are in millimeters:

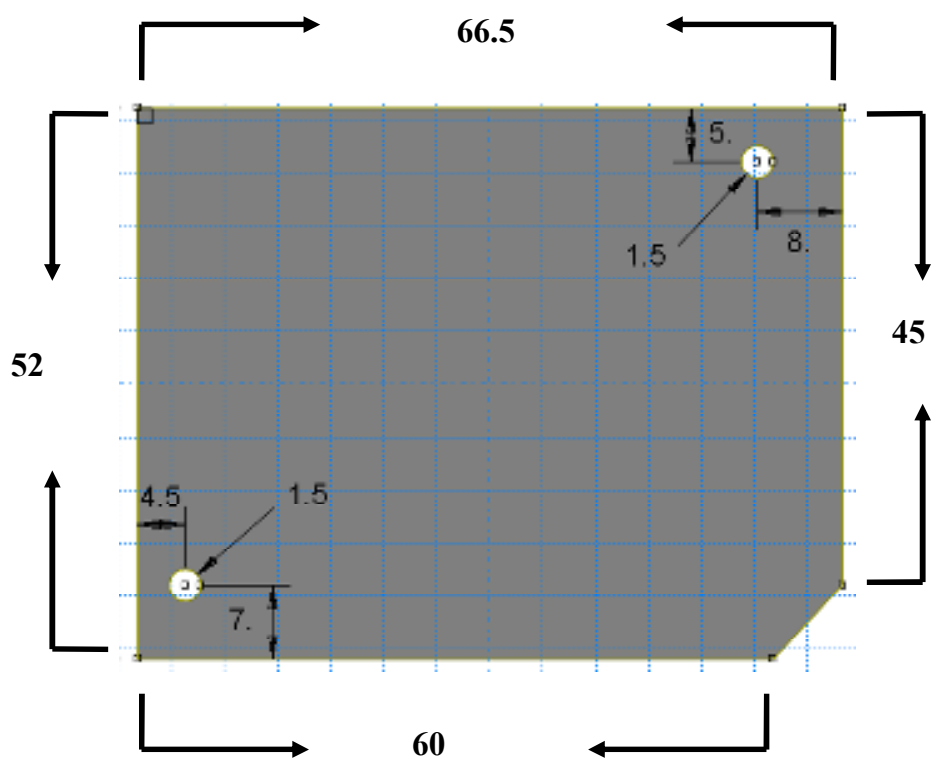

Figure A-1 PWB- Shell thickness is equal to 1.03

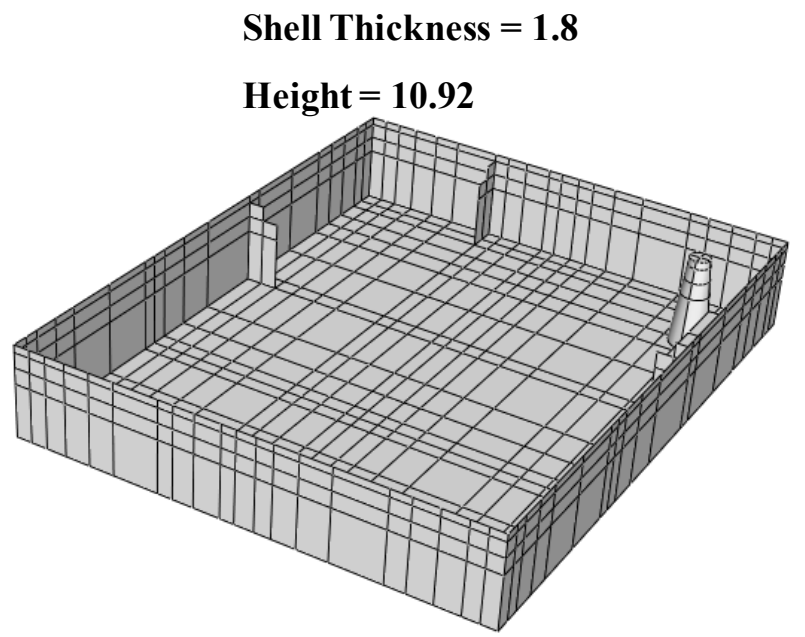

Figure A-2 Bottom Housing- Shell thickness is equal to 1.8 


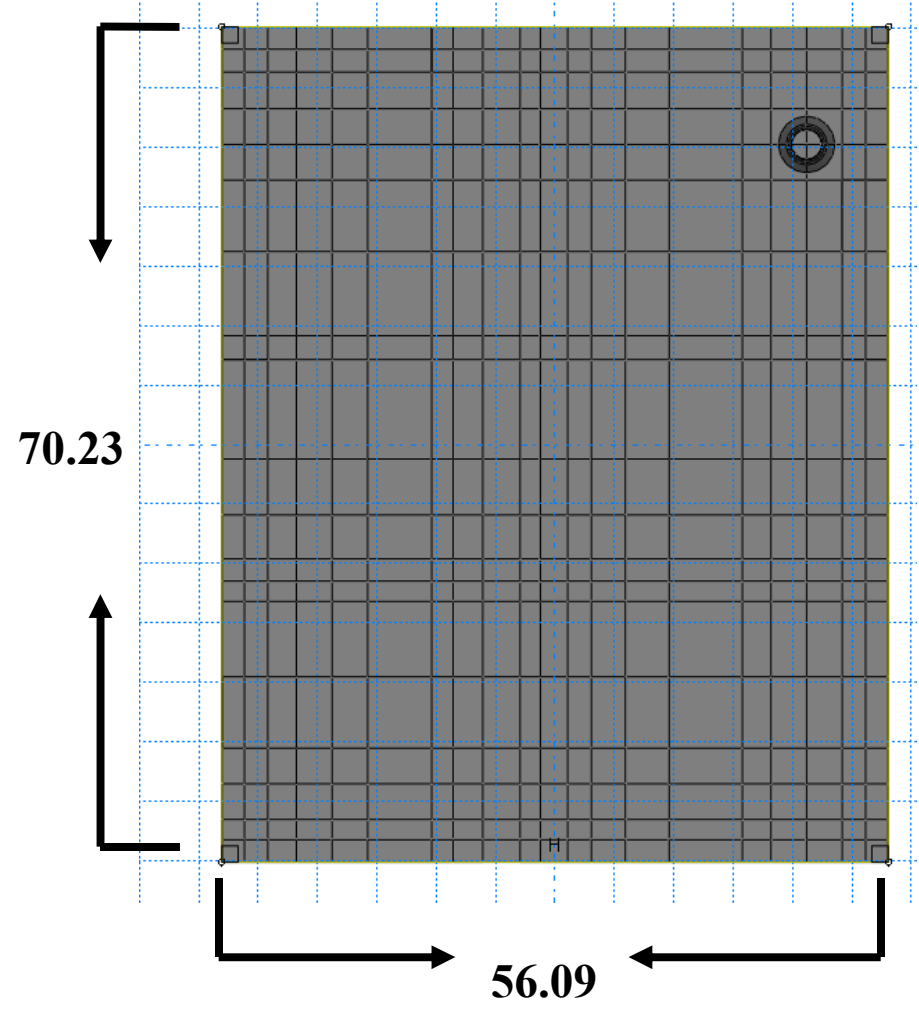

Figure A-3 Bottom housing top view

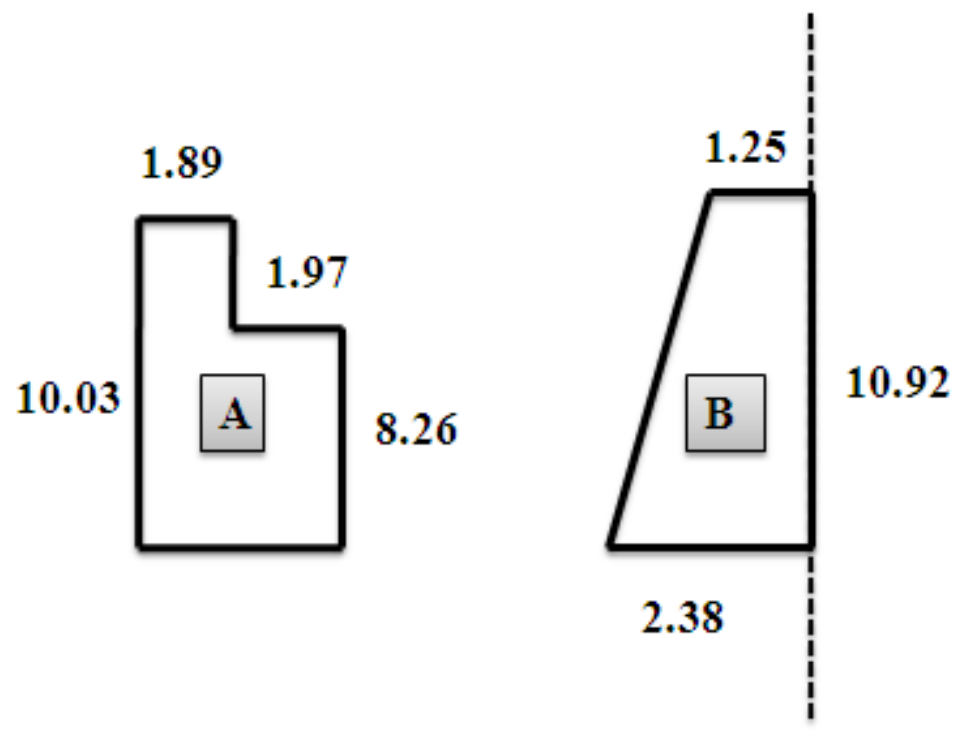

Figure A-4 Bottom housing A) Rib B) Tapered post 


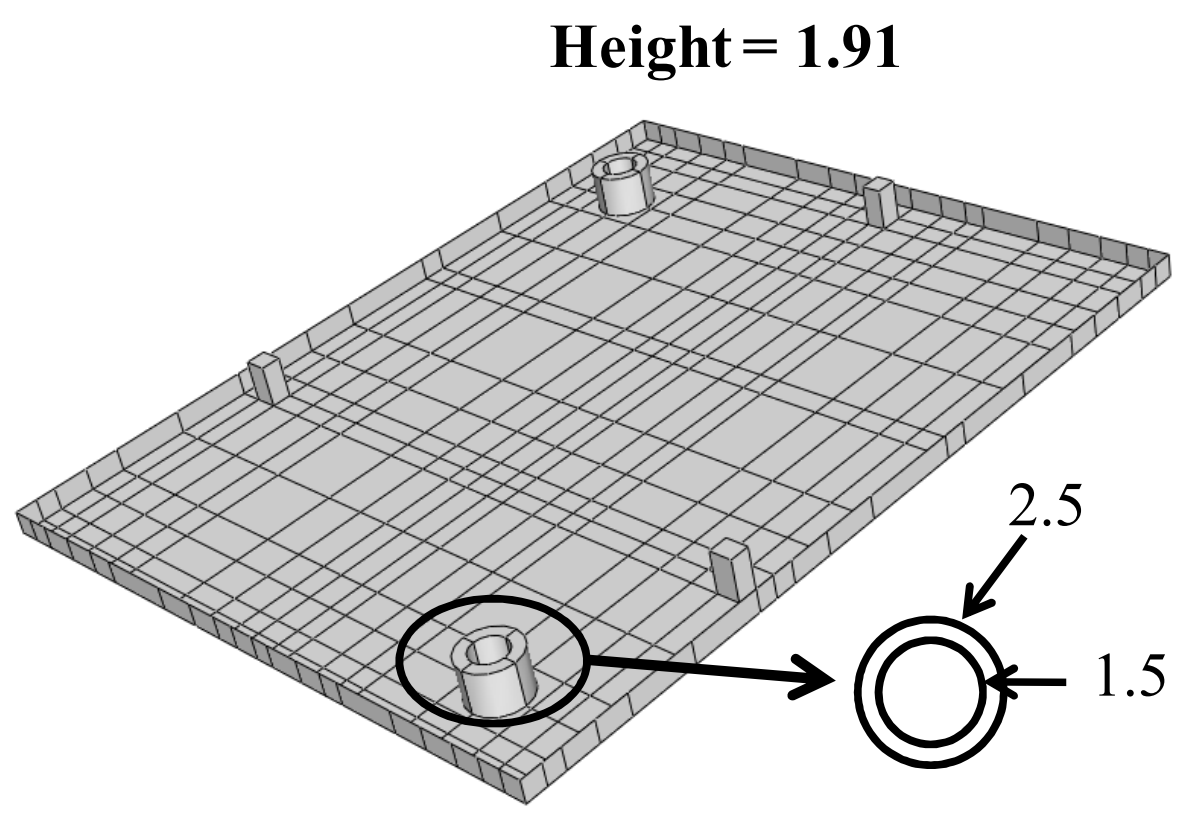

Figure A-5 Top housing

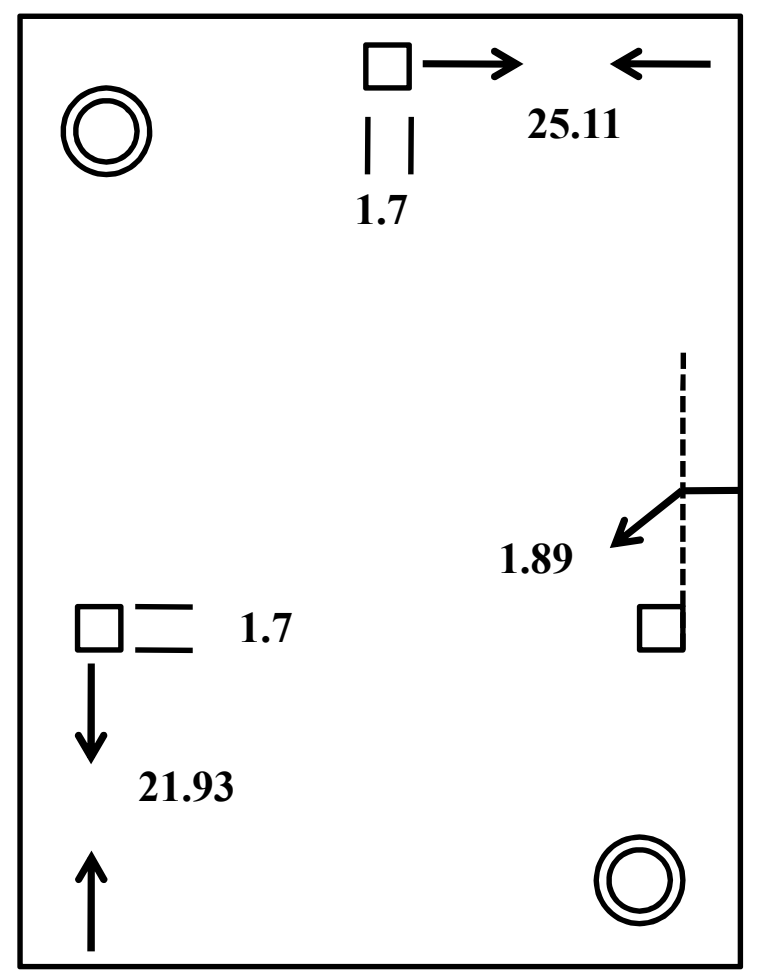

Figure A-6 Top housing top view 


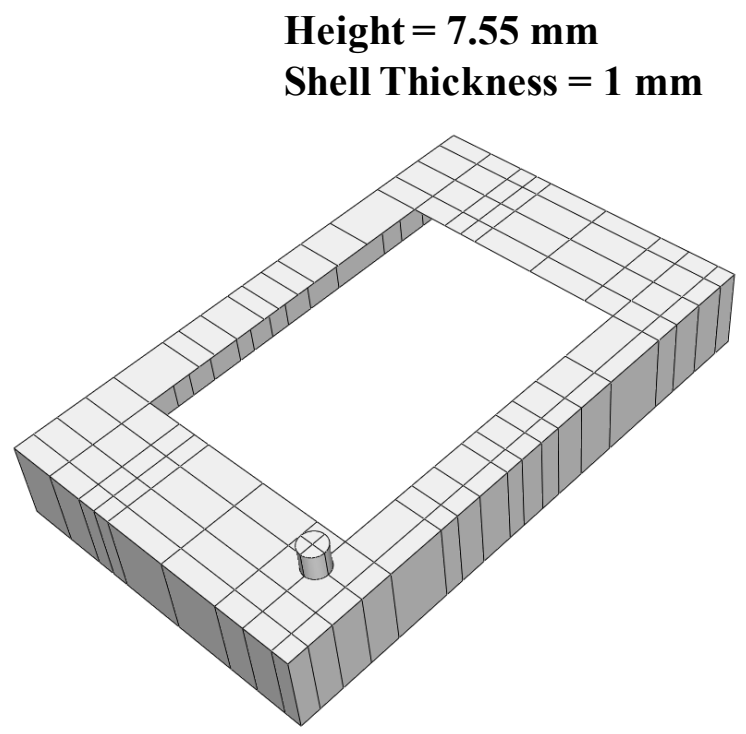

Figure A.7 Battery case-shell thickness is equal to $1 \mathrm{~mm}$

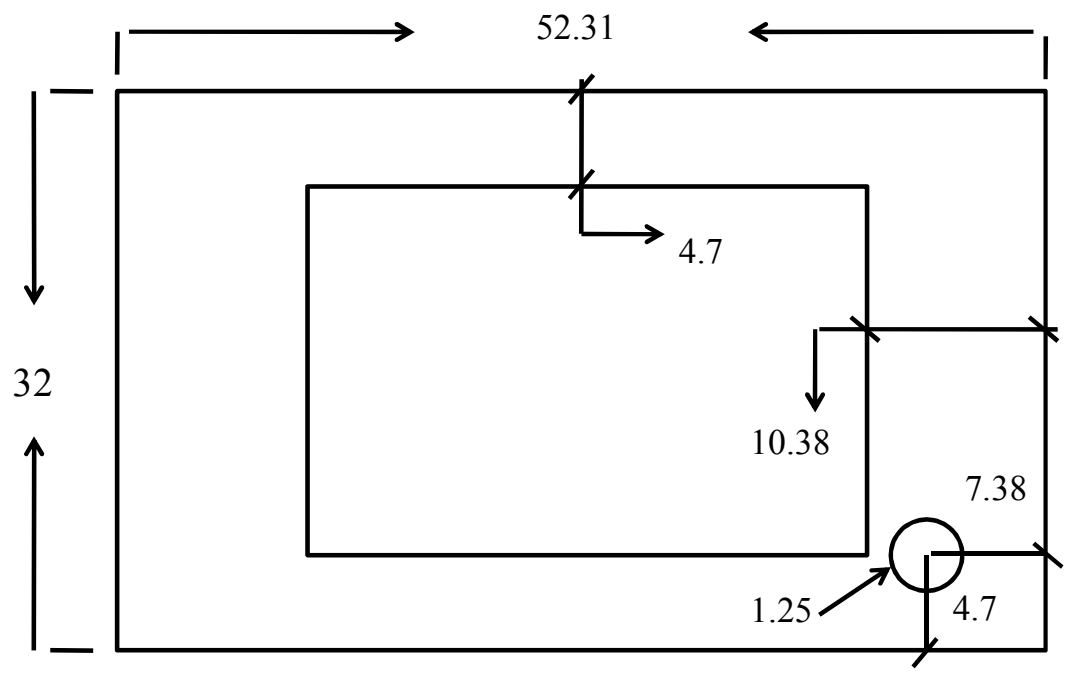

Figure A.8 Battery case top view 


\section{Appendix B Material Properties}

A) The PWB material defined in this study is FR4 and it is modeled as an orthotropic material.

Table B-1 FR4 Material properties [19]

\begin{tabular}{|c|c|}
\hline E1(MPA) & 19000 \\
\hline E2(MPA) & 19000 \\
\hline E3(MPA) & 9000 \\
\hline G12(MPA) & 3700 \\
\hline G21(MPA) & 3700 \\
\hline G13(MPA) & 2900 \\
\hline G23(MPA) & 2900 \\
\hline v12 & 0.14 \\
\hline v21 & 0.14 \\
\hline v13 & 0.39 \\
\hline v23 & 0.39 \\
\hline v31 & 0.18 \\
\hline v32 & 0.18 \\
\hline Dennsity & $1.80 \mathrm{E}-09$ \\
\hline
\end{tabular}

Table B-2 Modified FR4 material properties used in the simulations of Part II of the study

\begin{tabular}{|c|c|}
\hline E1(MPA) & 22800 \\
\hline E2(MPA) & 22800 \\
\hline E3(MPA) & 9000 \\
\hline G12(MPA) & 3700 \\
\hline G21(MPA) & 3700 \\
\hline G13(MPA) & 2900 \\
\hline G23(MPA) & 2900 \\
\hline v12 & 0.14 \\
\hline v21 & 0.14 \\
\hline v13 & 0.39 \\
\hline v23 & 0.39 \\
\hline v31 & 0.18 \\
\hline v32 & 0.18 \\
\hline Density & $1.80 \mathrm{E}-09$ \\
\hline
\end{tabular}


B) The Plastic housing material defined in this study is Lexan 500 and it is modeled as an isotropic material.

\section{Appendix C Shock Response of Spring-mounted PWB}

The PWB acceleration is magnified during shock loading of the spring supported PWB, similar to that seen in the case of the clamped PWB. The acceleration magnification factor is, however, marginally higher than that of clamped PWB. Figure C-1 demonstrates that the acceleration magnification factor is constant at 1.5 , for all acceleration levels. Moreover, Figure C-1 exhibits the repeatability of the shock test on the spring-mounted PWB.

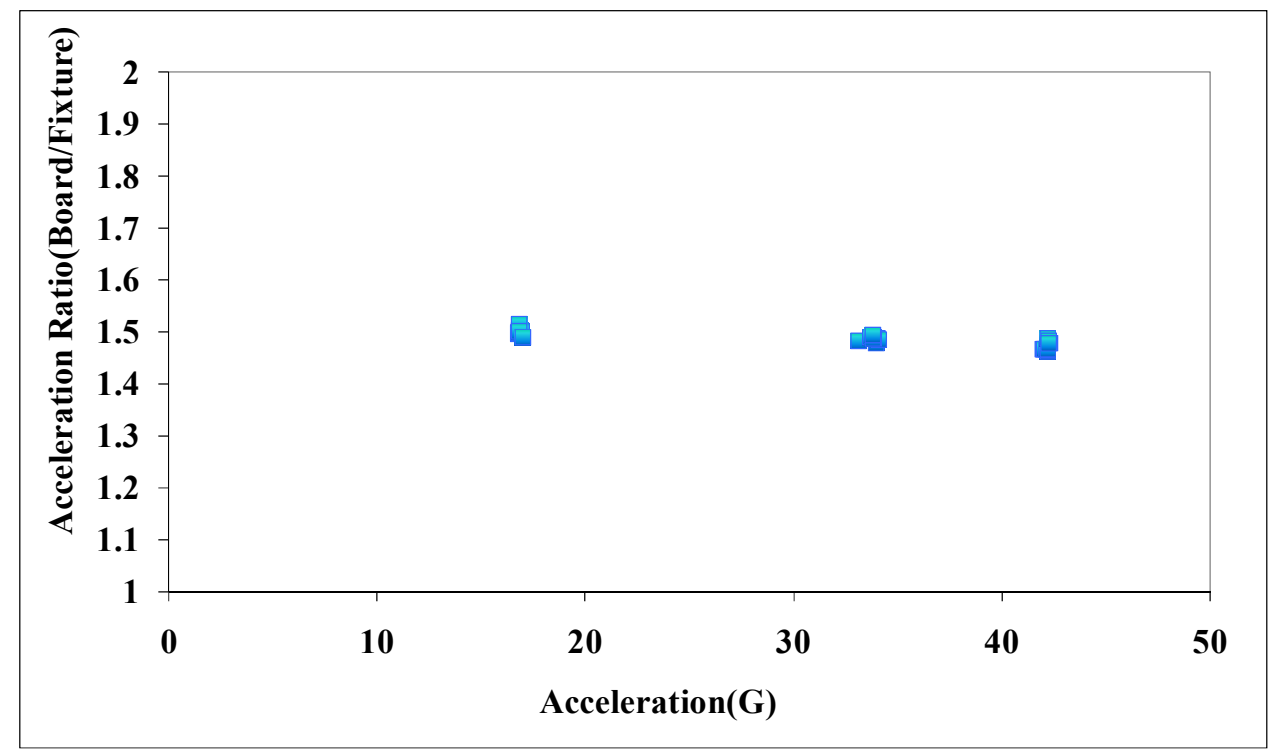

Figure C-1 Acceleration magnification factor for spring-mounted PWB

Figure C-2 compares the acceleration response of the spring mounted PWB at its free end as shown in Figure 2-11 with that of the fixture excitation. For the case shown in Figure C-2, PWB peak acceleration is 50 Gs for a fixture excitation of only 
33 Gs. Figure C-3 shows the strain history at the post of the spring mounted PWB for $35 \mathrm{G}$ excitation. The peak $\varepsilon_{\mathrm{yy}}$ strain at this location is 360 micro-strains.

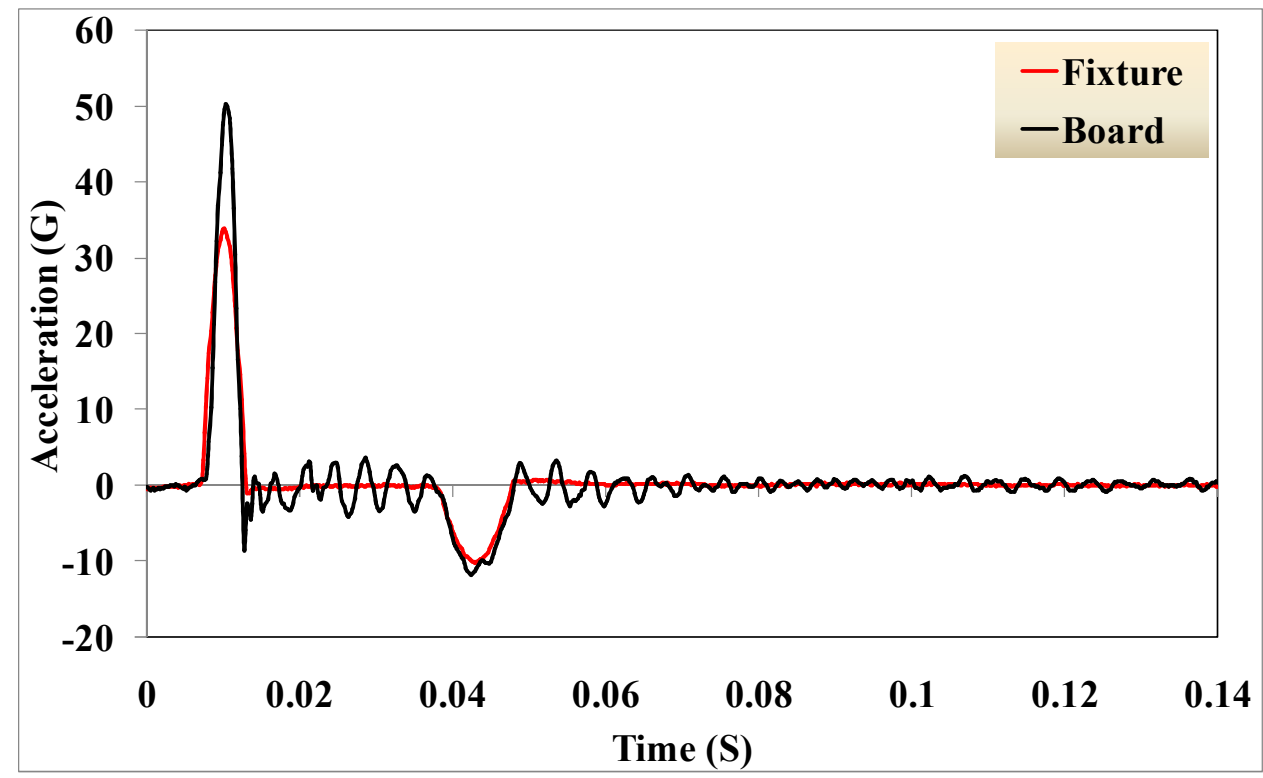

Figure C-2 Fixture acceleration VS spring-mounted board acceleration response measured at its free end

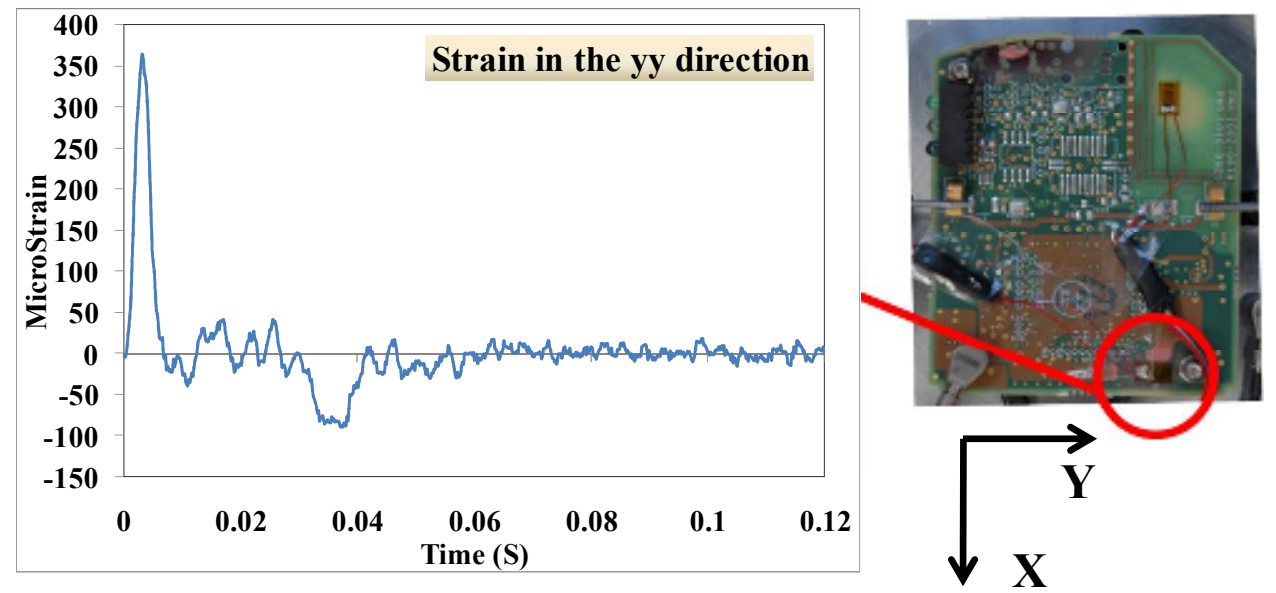

Figure C-3 Strain history $\varepsilon_{\mathrm{yy}}$ at the post of the spring-mounted board 


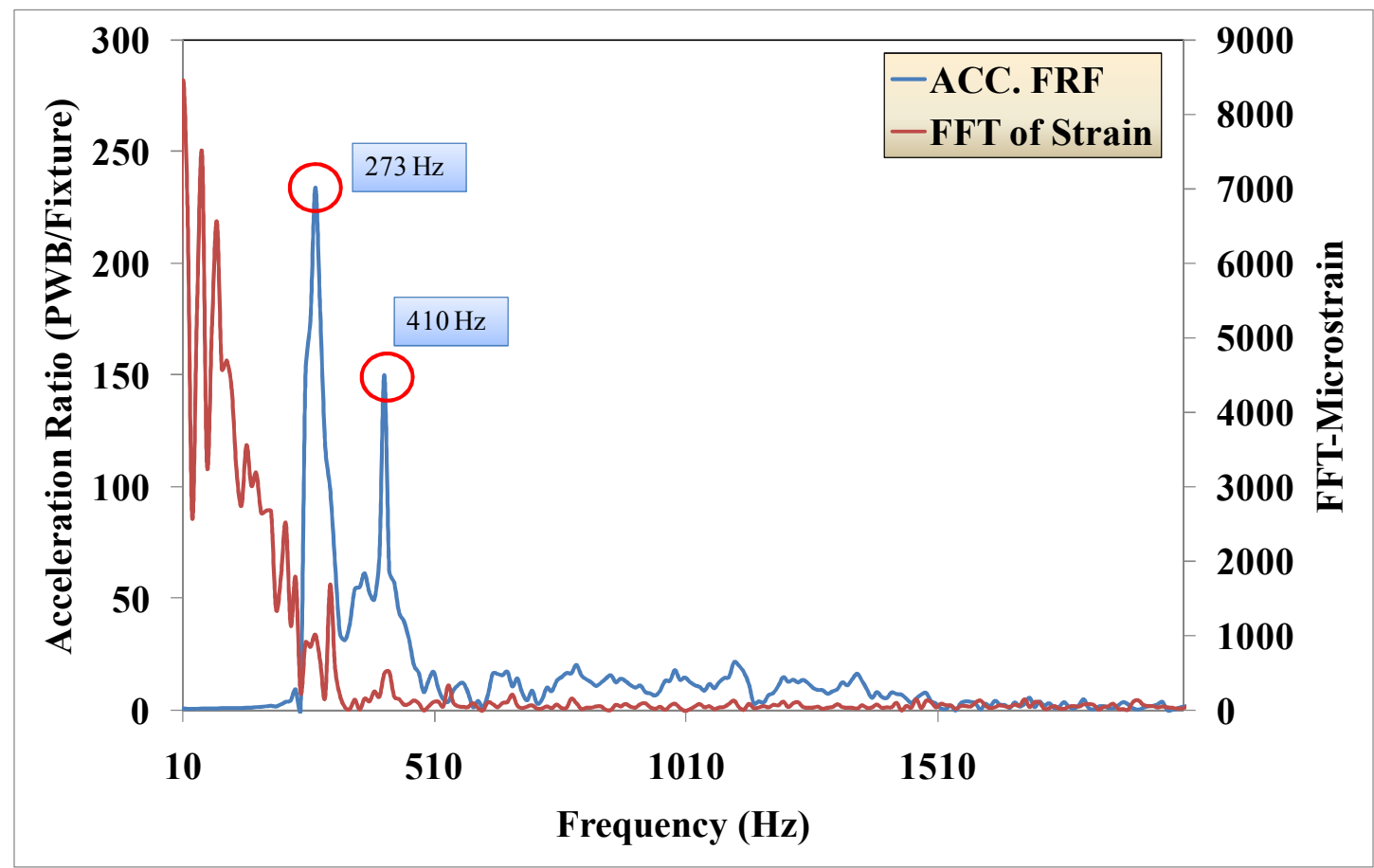

Figure C-4 Frequency response function of accelerometer B in Figure 2-11 in response to the half sine shock loading. FFT response of the strain gage, shown in Figure C-3.

As discussed in 2.5.1.1 adding the Au-plated springs to the board stiffens the system. Consequently, the first natural frequency of the spring mounted PWB no longer coincides with the notch on the excitation FFT. Therefore, unlike in the clamped PWB, the first resonant mode dominates the shock response of the springmounted PWB, as shown in Figure C-4. 


\section{Appendix D Finite Element Model of Spring-Mounted PWB}

In the case of the shock test model for the spring-loaded PWB, the boundary conditions for the input pulse must also be applied at the base of the Au-plated leaf spring locations, in addition to Points 1 and 2 (Refer to Figure 3-9). Thus, two elastic Cartesian spring connectors are used to tie these areas to Point 1, as shown in Figure 3-9. In the experimental part of the study (Part I), the first mode of the spring loaded board was seen to be the dominant response mode under shock loading, unlike in the case of the clamped PWB. Therefore, for the spring loaded shock simulation, the value of $\alpha$ is set to $69 \mathrm{Rad} / \mathrm{s}$, which corresponds to the first mode. Figure D-1 and Figure D-2 compare finite element simulation results with experimental measurements. These graphs indicate that, the model is able to predict the dynamic response of the spring loaded PWB under shock loading, with reasonable accuracy.

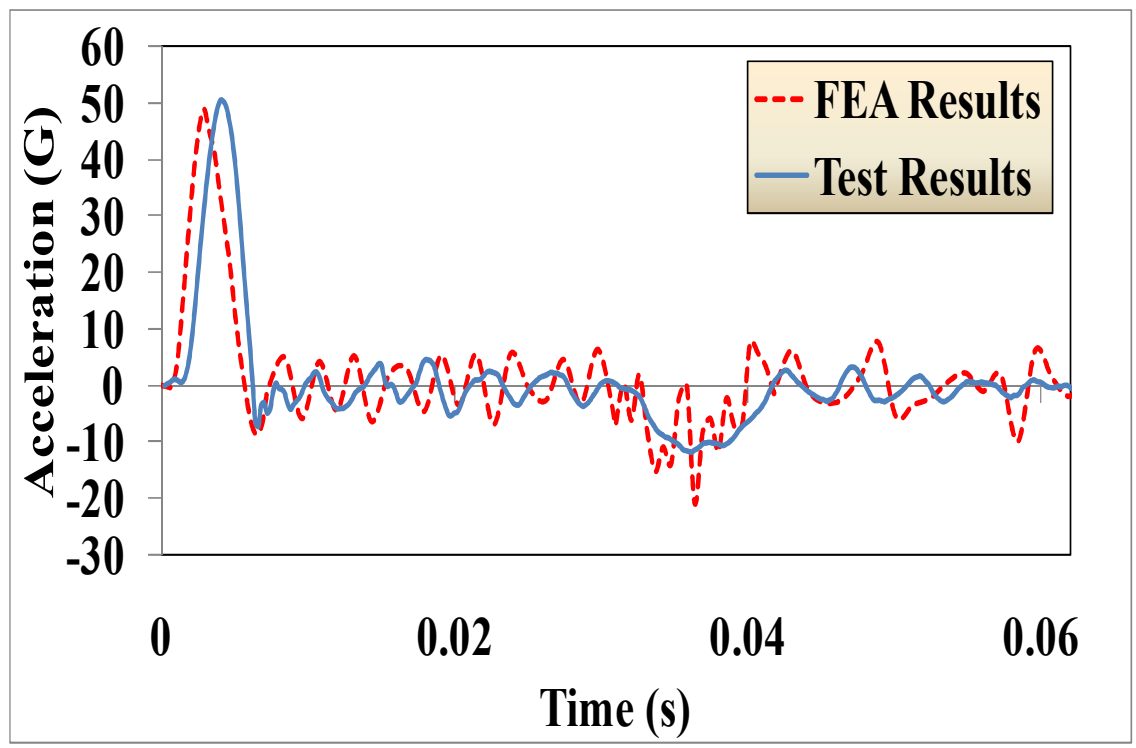

Figure D-1 Acceleration at the Free End of the Spring Loaded Board- FEA VS. Experiment 


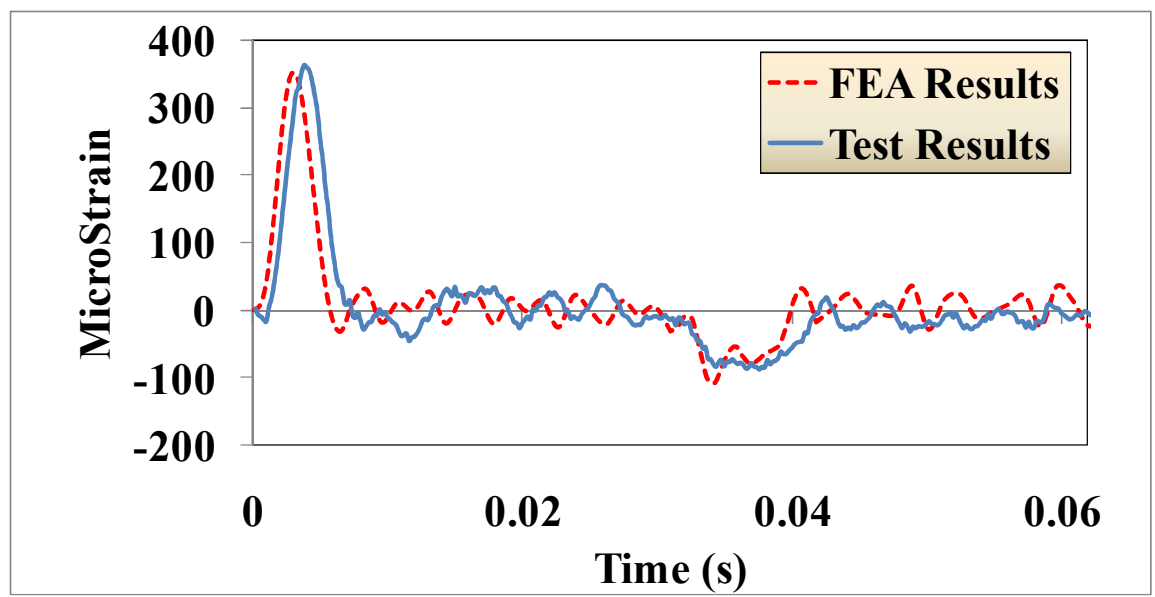

Figure D-2 Strain at the Post of the Spring Loaded Board- FEA VS. Experiment 


\section{Appendix E Test Set Up for Mechanical Property of Housing Material}

A separate broad-band test is used to identify the mechanical properties of the housing material. The test specimen is a small rectangular cantilever beam cut out of the top housing. The natural frequencies of the cantilever beam are identified from this broad-band test so that the Young's modulus of the Lexan 500 material documented can be obtained by finite element modeling and verified against the values documented in the literature, as discussed later in Part II of this study. In Section 2.3.4, the same broad-band test will be performed on the empty plastic housing. The goal is to investigate how accurately finite element modeling can predict the dynamic response of the plastic housing, based on the material properties obtained from the cantilever beam test. As shown in Figure E-1, the plastic beam is fixed at its end with aluminum bars. The aluminum bars are bolted to the base fixture with two bolts and several washers and nuts. There is a strain gage on the plastic beam close to the clamped base since the flexural strain is highest at that location. The cantilever beam broad band test is conducted twice. In the first trial, there is no accelerometer on the cantilever beam whereas in the second trial an accelerometer is placed on the free end of the beam (Accelerometer C) where acceleration is maximum. The change in natural frequency due to the mass of this accelerometer must be considered in the simulations presented later in Part II of this study. Another accelerometer is placed next to the bolt (Accelerometer B) to ensure that the bolt natural frequencies do not coincide with that of the cantilever beam. Furthermore, an 
accelerometer is placed on the base fixture (Accelerometer C) to control the shaker. A broad-band excitation of $0.01 \mathrm{~g}^{2} / \mathrm{Hz}$ is applied over a frequency range of $50 \mathrm{~Hz}$ $3200 \mathrm{~Hz}$. Additionally, the sampling rate for data acquisition is set to 8192 samples per second.

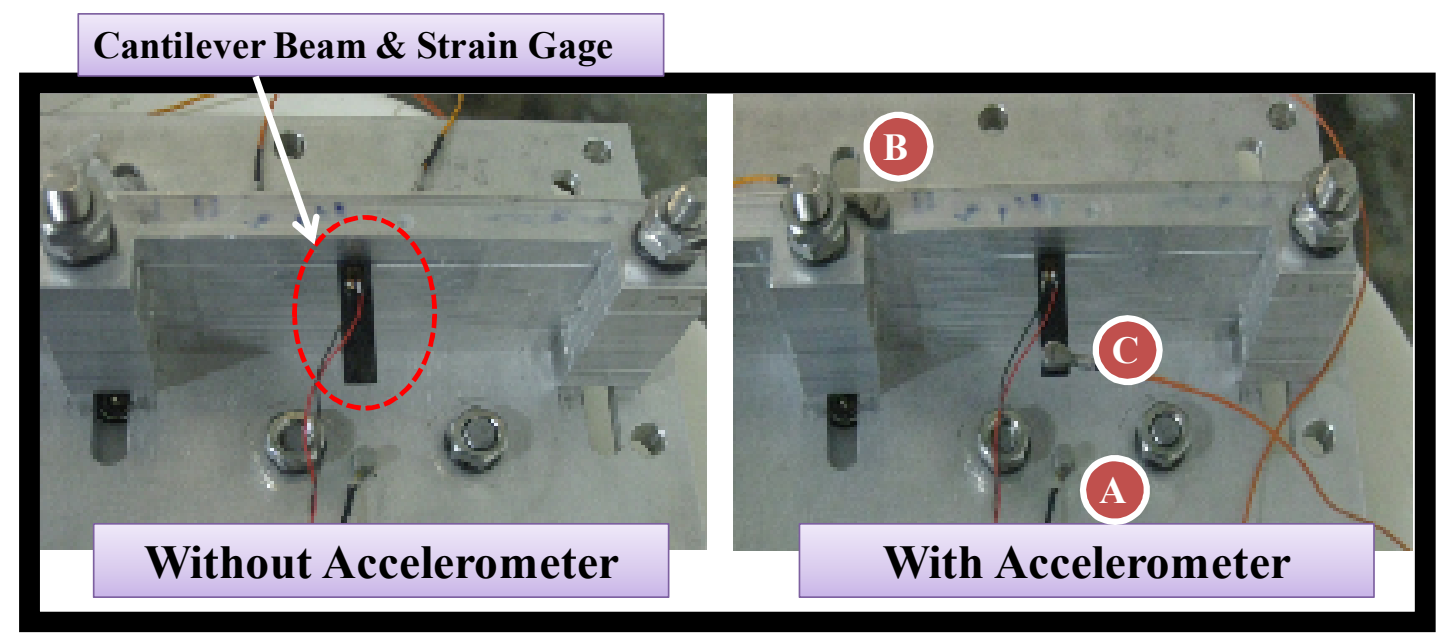

Figure E-1 Cantilever beam broad band test set up. The test specimen and the strain gage are labeled with a red dotted circle. Accelerometer A controls the shaker. Accelerometer B measures the bolt natural frequencies. Accelerometer $\mathrm{C}$ measures the plastic beam response. 


\section{Appendix F Test Set Up for Mechanical Property of Housing Material}

This section presents the natural frequencies of the cantilever beam constructed from the housing material. Figure F-1 illustrates the acceleration response of the cantilever beam, bolt, and the base fixture in the frequency domain. Additionally, Figure F-2 illustrates the transfer function between acceleration responses of the cantilever beam to the accelerometer mounted next to the bolt. As

shown in Figure F-1, the acceleration response of the base fixture is a constant line which implies that the natural frequencies of the base do not lie in 0-2500 frequency range. On the other hand, the accelerometer on the clamping mechanism clearly indicates many resonant modes, starting from about $800 \mathrm{~Hz}$. The accelerometer at the tip of the cantilever shows a clear peak at $154 \mathrm{~Hz}$ and many more beyond $800 \mathrm{~Hz}$. In the frequency range denoted by the dashed rectangle (beyond $800 \mathrm{~Hz}$ ), all the peaks from the accelerometers on the bolt and cantilever tip coincide with each other. Thus, it is difficult to conclude with certainty whether any of the cantilever response peaks except for the first peak at $154 \mathrm{~Hz}$ are due to its higher resonant modes or due to excitation peaks caused by the resonant modes of the clamping system. Therefore, for purposes of simplicity, the focus of this study will be only on the first peak at $154 \mathrm{~Hz}$, which is clearly the first resonant mode of the cantilever (with the mass of the accelerometer included at its tip). 


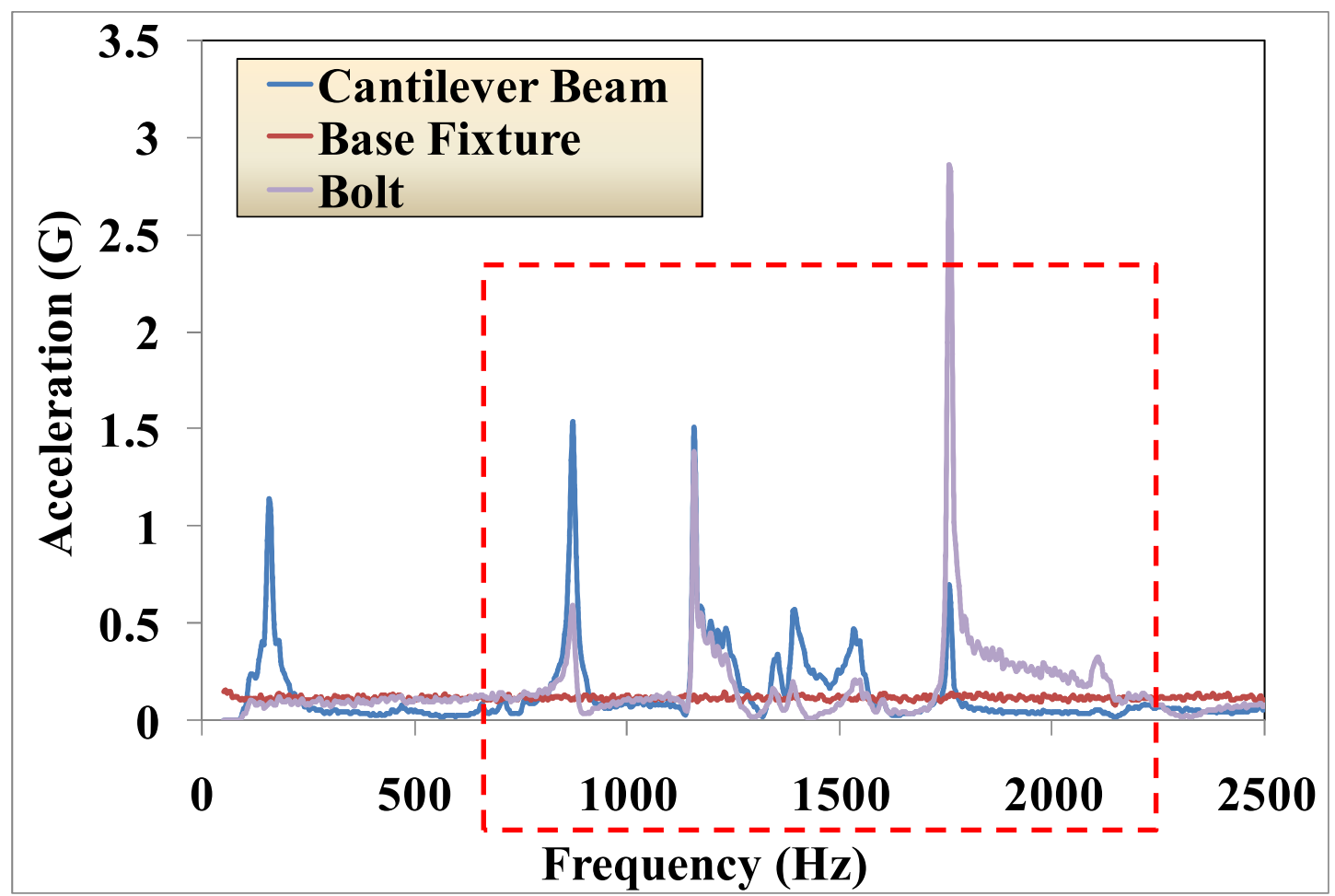

Figure F-1 Acceleration response of the cantilever beam, bolt, and the base Fixture in the Frequency domain. Refer to Error! Reference source not found. for accelerometer location.

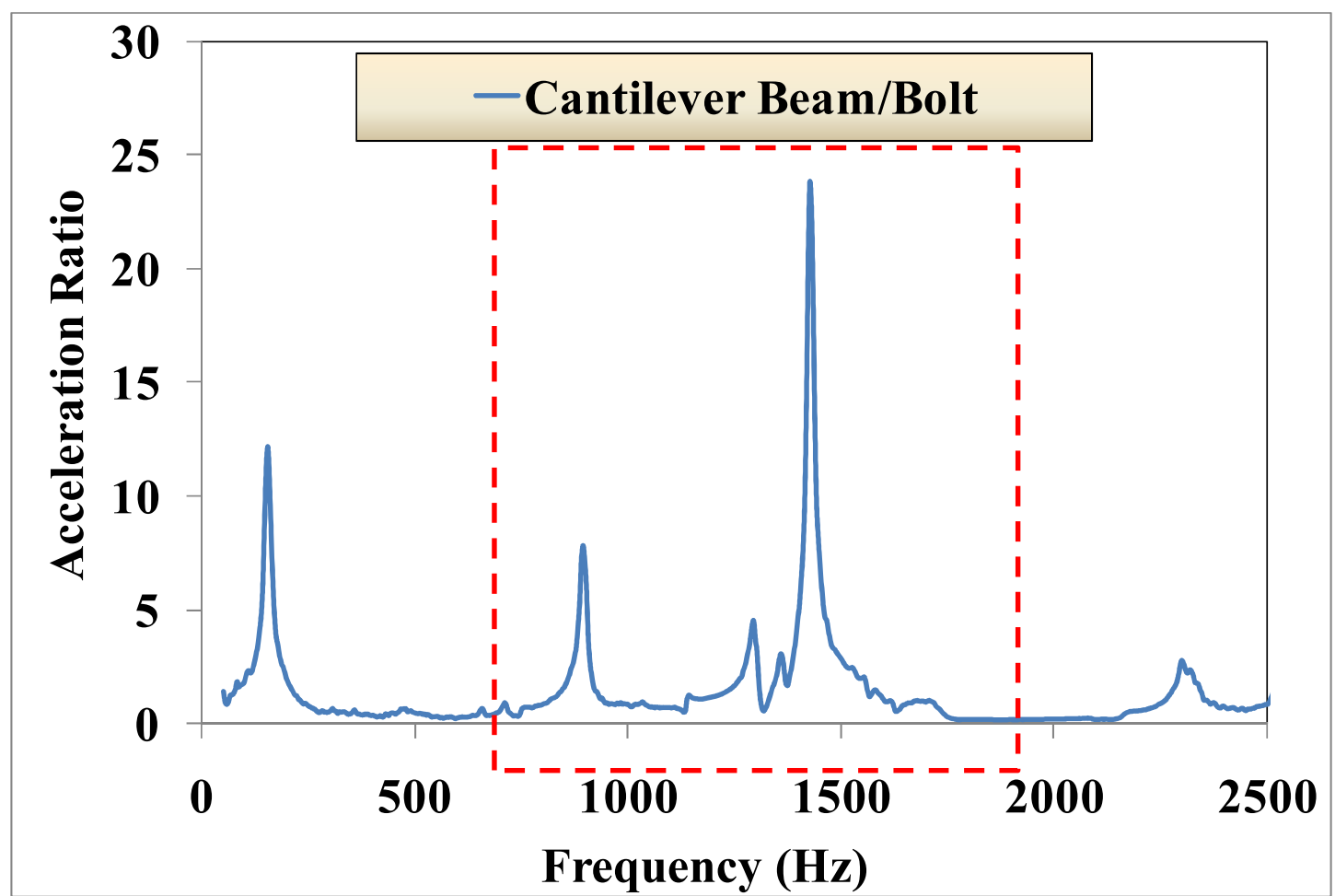

Figure 0F-2 Transfer function between the acceleration response of the cantilever beam versus the accelerometer next to the fixture bolt. Refer to Error! Reference source not found. for accelerometer locations. 
Figure F-3 demonstrates the FFT response of the strain gage on the cantilever beam when there is no accelerometer at its free end. The first resonant mode clearly occurs at $459 \mathrm{~Hz}$. This resonant frequency is almost thrice that without the accelerometer mass at the free end and these values will be used in Part II of this study to deduce the material properties of the plastic housing material that was used in the cantilever beam. Additionally, it is important to note that unlike the first cantilever response peak, other peaks beyond the first one did not change when accelerometer was removed from the cantilever beam as shown in Figure F-3Error! Reference source not found.. This clearly, indicates that the peaks beyond the first one are due to excitation peaks caused by the resonant modes of the clamping system.

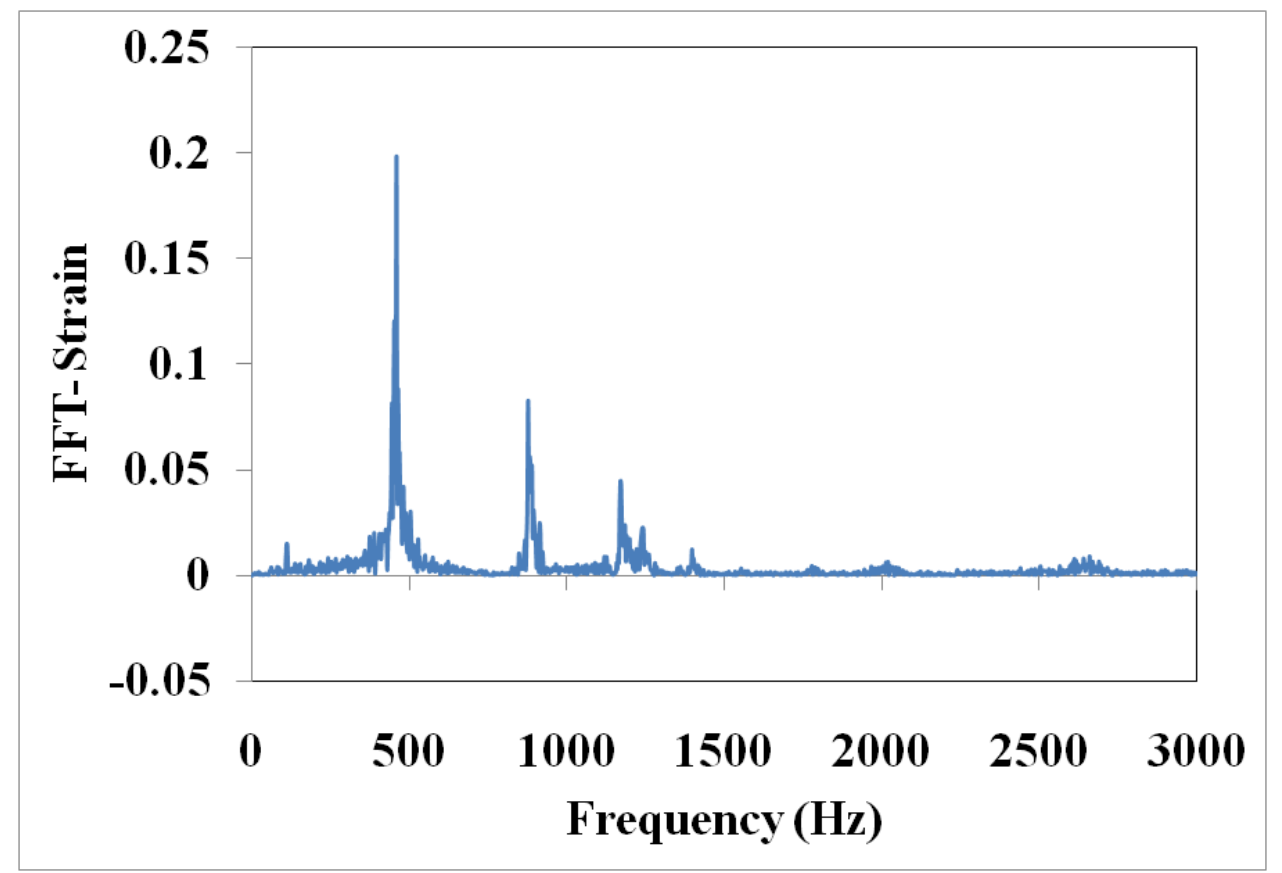

Figure F-3 FFT response of the strain gage shown in Figure 2-11, in response to broad band excitation 


\section{Appendix G Finite Element Modal Analysis of Cantilever Beam for Housing Material Properties}

As mentioned earlier in Section 2.3.3 of Part I of the study, material properties

for the housing material, extracted from the literature are verified by modal analysis of a cantilever beam constructed from the housing material. The modulus of elasticity obtained from this modal analysis in this section will be used in the next section when analyzing the empty case plastic housing to investigate the damping coefficient for the dynamic response of the empty case. Four-node quadrilateral, stress/displacement shell elements with large-strain formulation are used to create the geometry of the plastic cantilever beam. The Lexan 500 material of the housing used in the cantilever beam specimen is modeled as an isotropic material, with Young's modulus of $3450 \mathrm{MPa}$, as reported in the literature [20]. In accordance with the experiments reported in Section 2.3.3 in Part I of this study, finite element modal analysis is first performed with the mass of the accelerometer added to the tip of the cantilever beam model and subsequently without the mass of the accelerometer for the experiment that had only a strain gage on the beam. FEA modal analysis of the cantilever beam with the accelerometer shows the fundamental frequency to be 165 $\mathrm{Hz}$, which is also close to the experimental value of $154 \mathrm{~Hz}$. The first natural frequency of the cantilever beam without the accelerometer mass is predicted to be $450 \mathrm{~Hz}$, which is reasonably close to the experimental value of $459 \mathrm{~Hz}$. Thus, the material stiffness obtained from the literature for the plastic Lexan 500 material is found to match well with the test results. Furthermore, the value of damping $(\alpha)$, obtained by matching the FEA and experimental acceleration amplitudes is found to 
be $\alpha=110 \mathrm{Rad} / \mathrm{s}$. First mode shape and frequency response function of the plastic beam are shown in Figure G-1 and Figure G-2.

\section{First Mode Shape}

Figure G-1 First mode shape of the plastic beam

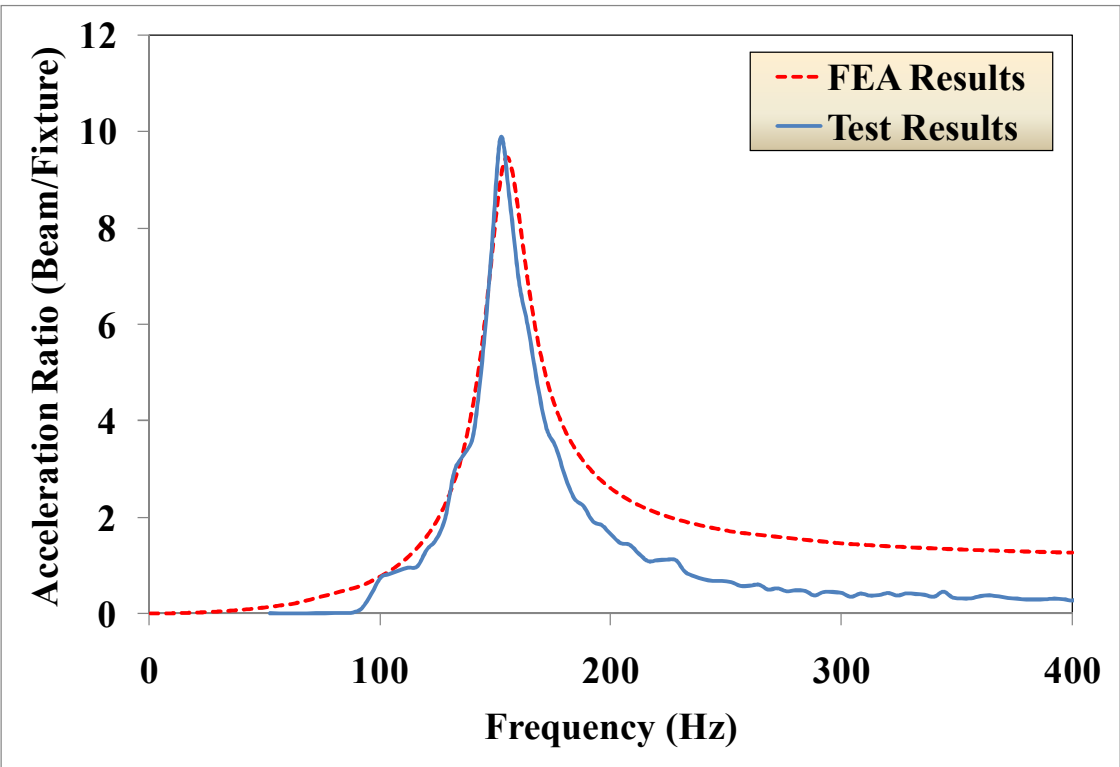

Figure G-2 Frequency response function for transverse acceleration at the tip of the cantilever beam 


\section{References}

[1] Seah S.K.W, Lim C.T, Wong E.H, Tan V.B.C, "Mechanical Response of PCBs in Portable Electronic Products during Drop Impact", Institute of Microelectronics Singapore, Electronics Packaging Technology Conference, IEEE, 2000.

[2] Tan L.B., Ang C.W., *Lim C.T., V.B.C.Tan, "Modal and Impact Analysis of Modern Portable Electronic Products", Institute of Microelectronics Singapore, Electronics Packaging Technology Conference, IEEE, 2005.

[3] Lim, C.T., Ang, C.W., Tan, L.B., Seah, S.K.W., and Wong, E.H., "Drop Impact Survey of Portable Electronic Products", Proceedings of the 53rd Electronic Components and Technology Conference, pp. 113-120, IEEE 2003.

[4] Jing-en Luan ", Tong Yan Tee a, Eric Pek, Chwee Teck Lim, and Zhaowei Zhong, "Modal Analysis and Dynamic Response of Board Level Drop Test", National University of Singapore, Electronics Packaging Technology Conference, IEEE 2003.

[5] Pekka Marjamäki, "Vibration test as a new method for studying the mechanical reliability of solder interconnections under shock loading conditions" Helsinki University of Technology, Laboratory of Electronics Production Technology, ISSN 1457-0440; 17, 2007.

[6] Balakumar Balachandran, Edward B. Magrab, Vibrations, Second Edition, Cengage Learning, 2009.

[7] S. Goyal, S. Upasani, and D. M. Patel, "Improving impact tolerance of portable electronic products: Case study of cellular phones," Exper. Mech., vol. 39, no. 1, pp. 43-52, Mar. 1999. 
[8] Chwee-Teck Lim, Y. M. Teo, and V. P. W. Shim, "Numerical Simulation of the Drop Impact Response of a Portable Electronic Product", IEEE Transactions on components and packaging technologies, Vol. 25, NO. 3, September 2002.

[9] Tee, T.Y., Ng, H.S., Lim, C.T., Pek, E., and Zhong, Z., 2004 "Impact Life Prediction Modelling of TFBGA Packages under Board Level Drop Test" Microelectronics Reliability.

[10] Scott Irving and Yong Liu, "Free Drop Test Simulation for Portable IC Package by Implicit Transient Dynamics FEM", Electronic Components and Technology Conference, IEEE 2004.

[11] Jing-en Luan and Tong Yan Tee, "Novel Board Level Drop Test Simulation using Implicit Transient Analysis with Input-G Method”, Electronics Packaging Technology Conference, IEEE 2004.

[12] Sheng Liu, Xiaojun Wang, Bin Ma, Zhiyan Gan, Honghai Zhang, "Drop Test and Simulation of Portable Electronic Devices" , IEEE 6th International Conference on Electronic Packaging Technology 2005.

[13] Jason Wu, "Global and Local Coupling Analysis for Small Components in Drop simulation”, Mechanical Technology Center, PCS, Motorola,

[14] I. Chowdhury, P. Dasgupta. "Computation of Rayleigh Damping Coefficients for Large Systems", Department of Civil Engineering, Indian Institute of Technology, India, 1998.

[15] Dally, James W., and Robert J. Bonenberger. Design Analysis of Structural Element. Fourth Edition. Knoxville, Tennessee: College House Enterprise, LLC, 2004. 
[16] ABAQUS/Explicit Users Manual, Hibbit, Karlsson \& Sorensen Inc., 2000.

[17] Enviromental Test Methods and Engineering Guidelines, MIL-STD-810E, July 1989

[18] JEDEC Solid State Technology Association, JESD22-B104-B: Mechanical Shock, 2001

[19] Sarvotham M. Bhandaraka, "Thermomechanical Analysis and Fatigue Life Cycle Prediction of Plated-Through Holes in Multilayered Printed Wiring Board" PhD Dissertation, Table 3.1, Page 82, 1992

[20] http://www.matweb.com/search/DataSheet.aspx?MatGUID=bf4bda349d264650 a5f7352bb1a7c827\&ckck=1

[21] Board Level Drop Test Method of Components for Handheld Electronic Products JEDEC JESD22-B111, 2003 